BERND GUTTING

\title{
DER EINFLUSS DER BESTEUERUNG AUF DIE ENTWICKLUNG DER WOHNUNGS- UND BAULANDMÄRKTE
}




\author{
BERND GUTTING
}

\title{
DER EINFLUSS DER BESTEUERUNG AUF DIE ENTWICKLUNG DER WOHNUNGS- UND BAULANDMÄRKTE
}

Am dynamischen Modell eines Marktsystems wird der Einfluß des bundesdeutschen Steuerrechts auf wohnungswirtschaftliche Entscheidungen gezeigt. Geltende steuerrechtliche Bestimmungen werden dabei auf ihre Bedeutung für Finanzierungs- und Investitionsentscheidungen unterschiedlicher Bauherrentypen untersucht. Auf der Grundlage seiner Ergebnisse formuliert der Autor Vorschläge für eine neutrale(re) Boden- und Wohnungsmarktbesteuerung.

Bernd Gutting wurde 1957 in Heidelberg geboren. Von 1977 bis 1982 Studium der Volkswirtschaftslehre an der Universität Mannheim; von 1982 bis 1985 wissenschaftlicher Mitarbeiter am Lehrstuhl für Volkswirtschaftslehre I an der Universität Mannheim. 
Der Einfluß der Besteuerung auf die Entwicklung der Wohnungs- und Baulandmärkte 


\section{STAATLICHE ALLOKATIONSPOLITIK IM MARKTWIRTSCHAFTLICHEN SYSTEM}

Herausgegeben von

Klaus Conrad, Heinz König, Hans-Heinrich Nachtkamp,

Rodiger Pethig, Ulrich Schlieper, Horst Siebert, Eberhard Wille

Band 22

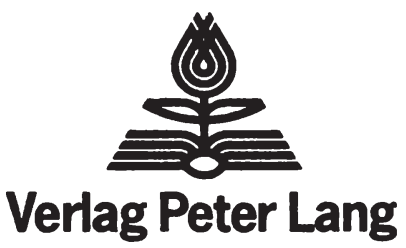

Frankfurt am Main · Bern · New York 


\section{BERND GUTTING}

\section{DER EINFLUSS \\ DER BESTEUERUNG AUF DIE ENTWICKLUNG \\ DER WOHNUNGS- UND BAULANDMÄRKTE Eine intertemporale Analyse der bundesdeutschen Gesetze}

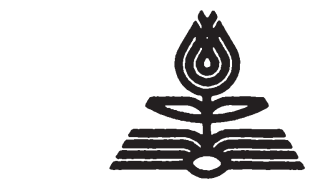

Verlag Peter Lang

Frankfurt am Main · Bern · New York 
CIP-Kurztitelaufnahme der Deutschen Bibliothek

\title{
Gutting, Bernd:
}

Der Einfluss der Besteuerung auf die Entwicklung der Wohnungs- und Baulandmärkte : e. inter= temporale Analyse d. bundesdt. Steuergesetze / Bernd Gutting. - Frankfurt am Main ; Bern ; New York : Lang, 1986.

(Staatliche Allokationspolitik im marktwirtschaftlichen System ; Bd. 22)

ISBN 3-8204-9506-1

NE: GT

Open Access: The online version of this publication is published on www.peterlang.com and www.econstor.eu under the international Creative Commons License CC-BY 4.0. Learn more on how you can use and share this work: http://creativecommons.org/licenses/ by/4.0.

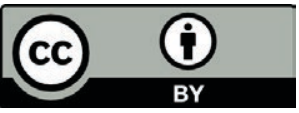

This book is available Open Access thanks to the kind support of ZBW - Leibniz-Informationszentrum Wirtschaft.

\author{
ISSN 0721-2860 \\ ISBN 3-8204-9506-1 \\ ISBN 978-3-631-75560-0 (eBook) \\ (C) Verlag Peter Lang GmbH, Frankfurt am Main 1986 \\ Alle Rechte vorbehalten.
}

Das Werk einschließlich aller seiner Teile ist urheberrechtlich geschützt. Jede Verwertung außerhalb der engen Grenzen des Urheberrechtsgesetzes ist ohne Zustimmung des Verlages unzulässig und strafbar. Das gilt insbesondere für Vervielfältigungen, Übersetzungen, Mikroverfilmungen und die Einspeicherung und Verarbeitung in elektronischen Systemen.

Druck und Bindung: Weihert-Druck GmbH, Darmstadt 


\section{INHALT SVERZE I CHIN IS}

I. EINFUHRUNG

1. Motivation und Aufgabenstellung

2. Ein allgemeiner Modellrahmen mit Wohnungs- und Bauland1 märkten

3. Zum methodischen Aufbau der Analyse

II. DIE LAISSER-FAIRE-ÖKONOMIE UND DAS ALLOKATIONSOPTIMUM

1. Das Planungsproblem des Wohnungsinvestors

1.1. Der optimale Finanzierungsplan

1.2. Der optimale Werterhaltungsplan

1.3. Der optimale Wohnungsneubauplan

2. Das Planungsproblem des Bodenmaklers

3. Bedingungen des Marktgleichgewichts - Charakteristika einer paretooptimalen Allokation

4. Die Dynamik der Läiisser-Faire-ökonomie

III. REAKTIONEN DES WOHNUNGSMAFKTES UND DES MARKTES FUR BAULAND AUF STEUERLICHE EINGRIFFE DES STAATES

1. Ubersicht der $z u$ analysierenden Steuerarten 85

2. Die Besteuerung des Immobilienvermögens

2.1. Die Grundsteuer

2.1.1. Die Grundsteuer geltenden Rechts 94

2.1.1.1. Einige institutionelle Details

2.1.1.2. Die Besteuerung baureifer Grundstücke und die Planungsprobleme von Bodenmaklern und Wohnungsinvestoren

2.1.1.3. Die Besteuerung bebauter Grundstücke und das Planungsproblem des Wohnungsinvestors

2.1.1.4. Die Besteuerung baureifer und bebauter Grundstücke als konzeptionelle Einheit

2.1.2. Die Bodenwertsteuer - die beste Lösung?

2.1.3. Eine allokationsneutrale Grundsteuer 
3. Die Besteuerung des Bodenverkehrs - ist die Grunderwerbsteuer tatsächlich eine Mobilitätsbremse?

4. Die Besteuerung der Mietumsätze

4.1. Die generelle Umsatzsteuerbefreiung

4.2. Die Mehrwertsteueroption

5. Die Besteuerung der Erträge des Immobilienvermögens

5.1. Ein allokationsneutrales System der Einkommens-

5.2. Die Realität - die bundesdeutsche Einkommensteuer

5.2.1. Die Einkommensbesteuerung des Bodenmaklers

5.2.1.1. Die Steuerfreiheit von Wertzuwächsen und das Phänomen der Baulandspekulation

5.2.1.2. Die Besteuerung realisierter Veräußerungsgewinne

5.2.2. Die Einkommensbesteuerung des Wohnungsinvestors

5.2.2.1. Die Einkommensbesteuerung in der Erwerbsphase: Käufer versus Bauherr

5.2.2.2. Die Besteuerung in der Nutzungsphase

5.2.2.2.1. Der Wohnungsinvestor als nicht-gewerblicher Vermieter

5.2.2.2.2. Der Wohnungsinvestor als Selbstnutzer

5.2.2.2.3. Uberschußrechnung und vorzeitiger Verkauf

5.2.2.2.4. Überschußrechnung und Nutzungswechsel

5.2.2.2.5. "Investitionsgutlösung" oder "Konsumgutlösung"?

5.2.2.2.6. Der Wohnungsinvestor als gewerblicher Vermieter

5.3. Perspektiven einer effiziente(re)n Form der Ertragsbesteuering des Wohnungssektors 
I .

EINLE ITUNG

\section{Motivation und Aufgabenstellung}

Daß Wohnen mit dem Lebensvollzug des Menschen untrennbar verbunden ist, ist eine Tatsache, über die zu diskutieren sich nicht lohnt.

Einer ausführlicheren Analyse wert sind dagegen die Schlußfolgerungen, die die Träger politischer Entscheidungen nicht nur unseres Landes aus diesem Sachverhalt in der vergangenheit gezogen haben und auch heute immer noch ziehen in keinem anderen Bereich unserer Volkswirtschaft, die Bereiche der Landwirtschaft, des Bergbaus und der Stahlindustrie vielleicht ausgenommen, sind die staatlichen Eingriffe so umfangreich wie im Wohnungssektor.

Die Interventionen der öffentlichen Hand erfolgen hierbei nicht nur auf dem Wege offener staatlicher Subventionen der erste und der zweite Förderungsweg des II. Wohnungsbaugesetztes (II.WoBauG) wären zwel Beispiele hierfür; auch verdeckte Eingriffe, die ihre Ursache in einer steuerlich differenzierten Behandlung des Wohnungssektors haben, sind ein sehr beliebtes Instrument selektiver staatlicher Förderung. Dabei erfährt nicht nur der Wohnungssektor insgesamt eine (steuerliche) Sonderbehandlung - auch innerhalb dieses Bereichs selbst werden unterschiedliche Investorgruppen im Rahmen des Steuer- und subventionssystems unterschiedlich behandelt.

Allerdings deuten Klagen von Wohnungsunternehmen über Leerstände im Mietwohnungsbestand und notleidende Bauherrenmodelle, die fast überall die Immobilienteile der Tageszeitungen füllen, darauf hin, daß vielerorts offensichtlich des "Guten" zuviel getan und produktive Ressourcen fehlgeleitet wurden. 
Daß solche Fehlallokationen ausnahmslos mit gesellschaftlichen Wohlfahrtseinbußen verbunden sind - das in notleidende Bauvorhaben investierte Kapital hätte in anderen sektoren gewinnbringend(er) eingesetzt werden können - ist eine ökonomische Lehrbuchweisheit

Tatsache ist allerdings auch, daß Aspekte der Allokationseffizienz bei politischen Entscheidungen - wenn überhaupt - nur eine untergeordnete Rolle spielen. Während Verteilungsfragen und Probleme der Wirtschaftsstabilisierung meist sehr ausfühlich und heftig diskutiert werden, läuft derjenige, welcher allokationstheoretische tuberlegungen in die Diskussion einzubringen versucht, Gefahr, als politischer Tagträumer belächelt oder gar der ideologischen Befangenheit bezichtigt $\mathrm{zu}$ werden.

Diese Kritik ist unbegründet; zum einen wird hierbei nicht bedacht, daß die materielle Wohlfahrt eines Landes im wesentlichen durch das Maß bestimmt wird, in dem es gelingt, die vorhandenen knappen Ressourcen in jene Bereiche $z u$ lenken, in denen sie den größten volkswirtschaftlichen Nutzen stiften; und es ist deshalb seit jeher auch das erste Anliegen des ökonomen, Antwort auf die Fragen zu geben,

- welche Güter und Leistungen in welchem Umfang, an welchem ort und $\mathrm{zu}$ welcher zeit bereitgestellt werden sollen, - welche Technologien bei der Produktion anzuwenden sind und - wie diese Güter und Leistungen auf die Menschen verteilt werden sollen.

Zum anderen wird bei dem Vorwurf der Ideologiebefangenheit der Umstand übersehen, daß im Streit der Wirtschaftssysteme nicht die Frage der Allokationseffizienz der eigentliche Zankapfel ist - auch sozialistische ökonomen wissen effi- 
ziente Allokationen durchaus zu schätzen ${ }^{1)}$ - sondern daß im Mittelpunkt der Auseinandersetzung die Frage steht, welche Organisationsform des volkswirtschaftlichen Prozesses das Problem des Erreichens des Wohlfahrtsmaximums am besten löst.

Ein mögliches Hemmnis auf dem Weg $z u$ einer optimalen Allokation könnte die staatliche Steuerpolitik sein. Ein Großteil unserer Steuern ist an das Eigentum an Vermögen bzw. an die aus diesem Vermögen fließenden Erträge geknüpft. Dabei spielt es für die Abgrenzung der jeweiligen Steuerbemessungsgrundlage oft eine entscheidende Rolle, in welchem Bereich unserer Volkswirtschaft dieses Vermögen gebunden ist und welchen juristischen Status das Steuergesetz demjenigen zuweist, der in den Genuß der Vermögenserträge kommt. Dies gilt in besonderem Maße für den Wohnungssektor: so wird beispielsweise die steuerliche Behandlung von Erträgen aus Wohnimmobilienvermögen davon abhängig gemacht, ob es sich bei dem Steuerpflichtigen um eine juristische Person oder um eine natürliche Person handelt, ob der Wohnungseigentümer seine Immobilie selbst nutzt oder vermietet und - wenn er Selbstnutzer ist - ob er dies als "normaler" privater Haushalt oder als Land- oder Forstwirt tut.

Dies sind nur wenige Beispiele aus einer ganzen Palette steuerlicher Differenzierungen, die es reizvoll machen $z u$ untersuchen, welche Einflüsse von der aktuellen Steuergesetzgebung ausgehen auf das allokative Geschehen auf den wohnungs- und Baulandmärkten. Dabei empfiehlt es sich, den schwerpunkt unserer Uberlegungen auf den sektor der freifinanzierenden Wohnungsinvestoren $z \mathrm{u}$ legen; denn dem öffentlich geförderten und auch dem steuerbegünstigten Wohnungsbau sind spezifische Förderziele - vor allem regional-, struktur- und ver-

1) Vgl. Z.B. ENGETS(1923), S.335 f., LANGE/TAYLOR(1948). 
teilungspolitischer Art - und dementsprechend Förderinstrumente immanent, die mit den allgemeinen und meist auch subtileren Selektionsmechanismen des Steuersystems in keinem unmittelbaren zusammenhang stehen.

Probleme können im Rahmen einer solchen Steuerwirkungsanalyse dann entstehen, wenn man sich nicht darauf beschränken möchte, Steuerwirkungen $\mathrm{zu}$ beschreiben, sondern darüberhinaus eine Wertung der gefundenen Inzidenzen vorzunehmen beabsichtigt. Denn Steuern weräen nicht nur zum Zwecke der Einnahmenbeschaffung erhoben - miteingewoben in das Netzwerk des Steuersystems sind zusätzlich Regelungen, die zu einem bestimmten Verhalten anregen oder vor einem solchen abschrekken sollen. So soll beispielsweise die Ertragsbesteuerung (auch) im Wohnungssektor mehrere Aufgaben gleichzeitig erfüllen: sie soll dem staat nicht nur Einnahmen verschaffen, sondern darüberhinaus die Vermögensbildung in Form von Wohneigentum fördern, das Ziel einer gerechteren Einkommensverteilung unterstützen helfen. Und auch die Grundsteuer wird nicht nur ceshalb erhoben, weil die hebeberechtigten Gemeinden ihr Aufkommen so dringend zur Konsolidierung ihrer Haushalte benötigen; vielmehr sollen auf diesem wege auch vermutete Unvollkommenheiten des Baubodenmarktes korrigiert werden.

Es ist dies nicht der geeigrete Ort, die Frage nach dem Für und Wider staatlicher Interventionen in den Wohnungsbau und die Wohnversorgung ausführlich und zufriedenstellend $\mathrm{zu}$ diskutieren 2). Dem aufmerksamen Zuhörer entsprechender Diskussionen vermittelt sich aber sehr schnell ein Eindruck darüber, wie auBerordentlich schwer es ist, diese Eingriffe mithilfe allokationtheoretischer Argumente generell zu rechtfertigen.

Doch selbst wenn man selektive Anreize nicht von vornherein und a limine ablehnt, sondern sie - auch im Hinblick auf

2) Sehr etngehend diskutieren diese Frage beispielsweise NACHTKAMP (1984b), WINKEIMANN (1985); man lese hierzu auch das Gutachten des Wissenschaftlichen Beirates beim Bundeswirtschaftsministerium (BUNDESMINISTER DER WIRISCHAFT (1982)). 
die Vielfalt ihrer Zielsetzung - akzeptiert, scheint es angemessen zu sein, sich bei der Analyse der steuerlichen Behandlung des freifinanzierten Wohnungsbaus auf die fiskalische Zwecksetzung dieser Besteuerung $z$ u konzentrieren und von allen zusätzlichen nicht-fiskalischen Anforderungen abzusehen.

Denn man braucht auch bei der Formulierung gewollt selektiv wirkender steuerlicher Regelungen einen Maßstab, init dessen Hilfe sich Anreize und Abschreckungen abschätzen und gegenüber "neutralen" Regelungen abgrenzen lassen. Daß es in der tagespolitischen Diskussion an einem solchen Maßstab oft genug mangelt, ist eine bedauerliche Tatsache. Sie wird insbesondere dann offensichtlich, wenn steuerliche Vorschriften - wie etwa die Absetzbarkeit der Schuldzinsen - als Förderungsmaßnahmen gedeutet werden, obwohl sie es in Wahrheit gar nicht sind.

Die Reduzierung der Besteuerungsanalyse auf die fiskalische Zwecksetzung der betreffenden Steuer vermag uns einen solchen Maßstab zu liefern. Insofern handelt es sich bei der vorliegenden Arbeit um eine positive Analyse, da sie zu beschreiben versucht, wie die Besteuerung wirkt.

Sie ist zugleich aber auch eine normative Analyse, da sie Urteile über den volkswirtschaftlichen wert einzelner steuern fällt. Referenzpunkt dieser normativen Aussagen ist das paretianische wohlfahrtskriterium. Akzeptiert man einmal die These, daß der marktwirtschaftliche Proze $B$ unter bestimmten, idealtypischen Bedingungen eine Laisser-Faire-రkonomie zu ihrem Wohlfahrtsmaximum hinführt, so muß man einer rein fiskalisch motivierten steuerpolitik mit der Forderung entgegentreten, daß sie eine solche Form der Besteuerung finden muß, die das das Marktgeschehen bestimmende Handeln der verschiedenen Marktakteure möglichst wenig beeinflußt (und so das Wohlfahrtsmaximum am wenigsten beeinträchtigt).

Als mögliche Ursachen für eine durch die Besteuerung ausgelöste Verhaltensänderung der Marktteilnehmer nennt die mikro- 
ökonomische Analyse den Einkommens - und den Substitutions effekt. Für die wohlfahrtstheoretische Beurteilung einer Steuer ist aber allein der Substitutionseffekt von Bedeutung. Nur er ist die Ursache von Wohlfahrtsverlusten in Form sogenannter excess burden. Wollte man bei der allokativen Analyse daneben auch noch der Tatsache der unterschiedlichen Aufkommenskraft verschiedener Steuern Rechnung tragen, würde man Gefahr laufen, die eigentlichen Unterschiede in den Steuerwirkungen $\mathrm{zu}$ verwischen.

In der folgenden Analyse wird deshalb ein öffentlicher Sektor unterstellt, der in jeder Periode zur Finanzierung eines Transferprogramms gegebenen Umfancis dem privaten Sektor auf dem Wege der Besteuerung liquide Mittel entzieht; es wird in jeder Periode ein Budgetausgleich angestrebt - Staatsverschuldung gibt es nicht. Die Bürger der betrachteten ökonomie müssen deshalb zwar zunächst die unvermeidlichen Finanzierungslasten der Steuer tragen, sie erhalten die entrichteten Beträge aber anschließend auf dem Transferweg wieder zurück; das verfügbare Einkommen der privaten Haushalte ändert sich per saldo nicht, der Einkommenseffekt ist neutralisiert.

Die oben erwähnten excess burden resultieren aus der Tatsache, daB der Fiskus Besteuerungsformen in seinem Steuerrepertoire hat, deren für die ökonomie nachteiligen Folgen über die reine Finanzierungsbelastung hinausgehen; diese $\mathrm{Zu-}$ satzlasten haben ihre Ursache in Konstruktionsfehlern der betreffenden Steuern, die es dem Steuerpflichtigen ermöglichen, durch eine Veränderung seines Verhaltens - die er in der steuerfreien Welt freiwillig nicht vorgenommen hätte - der Steuerlast zumindest partiell auszuweichen. Es ist ein Postulat der Theorie der optimalen Besteuerung, daß sich der öffentliche Sektor möglichst auf die Erhebung solcher Steuern beschränken sollte, die solche Schlupflöcher nicht bieten. 
Steuern, die keine direkten Substitutionseffekte zur Folge haben, werden in der steuertheoretischen Literatur als neutral bezeichnet. Wir wollen diese Terminologie auch in unserer Analyse benutzen. Das Ziel dieser Arbeit wird es sein, das aktuelle, für den freifinanzierten Wohnungsbau relevante steuerrecht auf nicht-neutrale steuerarten zu untersuchen und $\mathrm{zu}$ überlegen, ob und wie diese Steuern durch geeignete Gesetzesänderungen in neutrale umgewandelt werden könnten.

Die zahlreichen Überlegungen und empirischen Untersuchungen, die in den letzten Jahren zum Thema "Optimale Besteuerung" angestrengt worden sind, vollziehen sich typischerweise in einem statischen Rahmen. Einflüsse der Besteuerung auf die Investitionsplanung der Unternehmen und die Sparentscheidungen der privaten Haushalte lassen sich damit aber nicht angemessen analysieren. Aber obwohl sich diese Probleme nur im Rahmen einer dynamischen steuerwirkungsanalyse befriedigend behandeln lassen, sind entsprechende Modelle in der Literatur noch vergleichsweise selten (als Beispiele hierfür seien die Arbeiten von AUERBACH [1979, 1981, 1982], BOADWAY [1978], FELDSTEIN [1974, 1978], SCHULz [1982] und SINN [1982, 1984] genannt). Dieses Defizit ist umso erstaunlicher, als schon I.FISHER [1907, 1931] recht früh auf die Bedeutung der intertemporalen Betrachtungsweise hingewieser hat.

Die vorliegende Arbeit steht - nicht nur aus diesem Grunde, wie wir noch sehen werden - in der Tradition der FISHERschen Analyse und baut auf auf zwei grundlegenden Arbeiten von SINN [1982, 1984] zur intertemporalen Problematik der Kapitaleinkommens- und Bodenbesteuerung.

Darüberhinaus soll sie ein weiteres Defizit decken helfen. Unseres Wissens gibt es bis zum heutigen Tag keine Arbeit, die im Rahmen eines umfassenden, geschlossenen theoretischen Ansatzes - sei er nun statisch oder dynamisch - die Wirkungsweise der steuer- 
lichen Behandlung des Wohnungssektors in der Bundesrepublik Deutschland zu erklären versucht. Allenfalls gibt es ökonometrische Partialmodelle, die das Wohnungsnachfrageverhalten privater Haushalte erklären sollen. Meistens jedoch beschränken sich die Autoren auf eine rein verbale Argumentation, die der Komplexität der verschiedenen steuerlichen Vorschriften und der hieraus resultierenden, oft genug gegenläufigen Effekte zwangsläufig nicht gerecht werden kann; so läßt sich mit Hilfe des hier verwendeten Ansatzes eine ganze Reihe von Mißverständnissen aufdecken, die auch heute noch die herrschende Meinung über die allokativen Wirkungen bestimmter steuerlicher Regelungen bestimmen.

Nicht zuletzt aus diesem Grunde vermag die vorliegende Arbeit auch neue Denkanstöße zu liefern für eine dringend erforderlich scheinende Reform von (nicht nur) den Wohnungsund den Bodensektor betreffenden Teilen des bundesdeutschen steuersystems. Insbesondere werden wir uns im folgenden mit den Fragen beschäftigen,

- ob die Grundsteuer geltenden Rechts ein probates Mittel ist, vermutete Unvollkommenheiten des Bodenmarktes zu korrigieren;

- wie eine effiziente Besteuerung des Grund und Bodens aussehen sollte, damit der Bodenmarkt überhaupt störungsfrei funktionieren kann;

- welche allokativen Konsequenzen aus

- der neuesten Reform des Grunderwerbsteuergesetzes und

- der Streichung der "Mehrwertsteueroption" ab 1.1.1985 resultieren;

- ob die Pauschalierung des Nutzungswertes oder die Ubberschußrechnung das für den Wohnungseigentümer rentablere Verfahren der Einkommenermittlung darstellt;

- ob die "Konsumgut"-Lösung oder die "Investitionsgut"-Lö- 
sung den adäquaten Weg zu einer effizienten Besteuerung des selbstgenutzten Wohneigentums weist;

- ob, wann und wie Bodenwertzuwächse steuerlich erfaßt werden sollten, um den Anforderungen einer neutralen Besteuerung $z u$ genügen;

- ob es der gewerbliche Wohnungsinvestor immer und in jedem Fall schwerer hat als sein nicht-gewerblicher Konkurrent, Wohnimmobilienvermögen zu bilden.

Neben diesen Schwerpunktthemen wird darüberhinaus eine Vielzahl ökonomischer Detailprobleme angesprochen, über deren Existenz sich die politischen Entscheidungsträger entweder nicht bewußt sind oder deren allokative Trägweite sie nicht übersehen.

2. Ein allgemeiner Modellrahmen mit wohnungs- und Baulandmärkten

Ausgangspunkt der Arbeit ist die Úberlegung, daB in der volkswirtschaftlichen Analyse üblicherweise nicht (natürliche oder juristische) Personen betrachtet werden, sondern Funktionen Gegenstand der Untersuchungen sind. Diese Funktionen erstrecken sich typischerweise auf

- das zur-Verfügung-stellen von Produktionsfaktoren,

- die Produktion und den Verbrauch von Gütern,

- die Verwendung des dabei entstandenen Einkommens sowie

- die Bildung und Beleihung von Vermögen.

Dieses Prinzip besitzt auch im Rahmen dieser Arbeit Gültigkeit; es werden nicht Personen betrachtet, die - in welcher Weise auch immer - am Wohnungs- und Baulandmarktgeschehen beteiligt sind, sondern ökonomische Funktionen, die auf dieses Geschehen Einfluß nehmen. Diese funktionale Betrachtungsweise bietet den Vorteil, daß man jene Märkte eindeutig abgrenzen kann, die mit dem Wohnungssektor verknüpft sind. Darüber hinaus läßt sich anhand dieser Vorgehensweise zeigen, welche steuerliche Folgen die Zuordnung verschiedener Funk- 
tionen auf einen einzelnen Steuerpflichtigen nach sich ziehen kann.

Betrachten wir nun die mit der Nutzung von Wohnraum verbundenen Funktionen bzw. deren Träger im einzelnen:

Um Wohnraum nutzen zu können, muß dieser zunächst einmal geschaffen werden - es muB einen wohnungsinvestor geben, der das Produktionsmittel Wohnung nachfrägt, und es muß einen wohnungsproduzenten geben, der das Produktionsmittel Wohnung errichtet.

Auf den ersten Blick unterscheidet sich die Produktion einer Wohnimmobilie nicht wesentlich von der Produktion anderer Investitionsgüter. Hier wie dort müssen nicht nur Produktionsfaktoren optimal kombiniert werden, auch die Schaffung aller organisatorischen und finanziellen Voraussetzungen ist integraler Bestandteil der Funktion "Produktion".

Es gibt aber eine wesentliche Besonderheit, die die Produktion des Investitionsgutes "Wohnung" von der Produktion anderer Investitionsgüter abhebt. Sie besteht nicht darin, daß die Wohnung meist nicht von einem einzigen Unternehmen allein, sondern von mehreren Unternehmen zusammen erstellt wird - auch in anderen Sektoren wird auf Vorleistungen zurückgegriffen und werden Großprojekte von Konsortien in Angriff genommen. Die Besonderheit besteht vielmehr darin, daß die Unternehmensleitung beim Bau der Wohnung einer speziellen Figur übertragen wird, dem Bauherrn. Und diese Besonderheit hat, wie wir noch sehen werden, steuerliche Folgen - Folgen, die es ratsam erscheinen lassen, daß wir der Funktion des Bauherrn unsere besondere Aufmerksamkeit schenken ${ }^{3}$ ?

3) Für den umbefangenen Betrachter verwirrend ist hierbei sicherlich die Tatsache, daß ein Bauherr im Sinne des II.WoBauG nicht unbedingt auch ein Bauherr im Sinne des Einkamensteuerrechts ist. Da wir unsere Analyse auf den freifinanzierten Wohnungsbau begrenzt haben, ist für uns allein die einkammensteuerliche Definition von Interesse. Danach ist Bauherr derjenige, der das "umfassend zu verstehende Bauherrenwagnis zu tragen hat", d.h. der das Risiko des Scheiterns trägt, und der jederzeit die Möglichkeit hat, rechtlich und tatsächlich auf Planung und Ausführung Einfluß zu nehmen;- vgl. BMF-Schreiben vam 13.8.1981, BStBl.I 1981, S.604. 
Von Bedeutung wird in diesem Zusammenhang auch die Auffassung des Bundesgerichtshofes sein, wonach ein Wohnungsinvestor, so er den Status eines Bauherrn genießen will, keineswegs alle Führungsaufgaben im Zusammenhang mit der Errichtung des Gebäudes selbst erledigen muß - eine bei sogenannten "Bauherrenmodellen" gängige Regelung sieht beispielsweise die Einschaltung von Baubetreuern und Generalübernehmern vor.

Anstatt die Wohnung selbst $z u$ bauen bzw. auf eigenen Namen, eigene Rechnung und eigenes Risiko bauen zu lassen, kann der Wohnungsinvestor seine Wohnimmobilie auch von einem Wohnungsproduzenten käuflich erwerben; in diesem Falle wird er steuerlich nicht als Bauherr behandelt, die Bauherreneigenschaft ist jetzt dem Wohnungsbauunternehmer l= Bauträgerl zu eigen.

Ublicherweise wird man davon ausgehen können, daß der Eigentümer der Wohnung diese auch wirtschaftlich verwertet, indem er sie entweder vermietet oder selbst bewohnt.

Um MiBverständnissen vorzubeugen,'muß an dieser stelle darauf hingewiesen werden, daB die in der Literatur häufig geäuBerte Behauptung, die Wohnung sei einerseits Investitionsgut - da Medium der vermögensbildung - andererseits aber auch Konsumgut - da durch den darin wohnenden Haushalt genutzt - aus ökonomischer sicht unzutreffend ist. Tatsächlich handelt es sich bei der wohnung um ein Produktionsmittel und in diesem Sinne auch um ein Investitionsgut - mit dessen Hilfe bestimmte, konsumierbare Leistungen erstellt werden, die in der Literatur als wohnungsnutzungen oder auch als wohnleistungseinheiten bezeichnet werden ") Insofern teilt auch das Investitionsgut "Wohnung" das für Produktiorsmittel charakteristische Schicksal intermediärer Güter, im Laufe der zeit in Konsumgüter transformiert $z u$ werden. Die beiden

4) So z.B. bei MUTH(1960), OLSON(1969), ARCELUS/METZLER(1973). 
einzigen bedeutsamen Merkmale, durch die sich die Produktion von wohnleistungen von der Produktion anderer Konsumgüter unterscheidet, bestehen darin, daß das produktionsmittel "Wohnung" permanent Wohnleistungen produziert, unabhängig davon, ob diese auch genutzt werden, und daß die "zuviel" produzierten wohnleistungen weder räumlich (Transport) noch zeitlich (Läger) transformiert werden können.

Prinzipiell wäre es völlig ausreichend, im Zusammenhang mit der Nutzung von Wohnraum zwischen den beiden Funktionsträgern Wohnungsinvestor und Wohnungsnutzer zu unterscheiden. Diese Differenzierung würde sowohl den Fall der Vermietung an einen anderen Haushalt als auch den der selbstnutzung umschliessen.

Nicht Rechnung tragen ließe sich dadurch aber einer spezifischen Eigenart des deutschen Umsatzsteuerrechts, das an die entgeltliche Uberlassung des Wohnungseigentums an einen gewerblichen Zwischen(ver)mieter ganz spezielle Steuerrechtsfolgen knüpft(e). Um die allokativen Konsequenzen des $\$ 9$ USt:G (alte Fassung) gebührend würdigen zu können, sei deshalb der Vermieter als weiterer Funktionsträger in unsere Analyse aufgenommen.

wohnungsinvestor, Bauherr, Vermieter und Wohnungsnutzer sind diejenigen Träger volkswirtschaftlicher Funktionen, die in das wohnungswirtschaftliche Geschehen am engsten involviert sind. Um unseren Modellrahmen abzurunden, müssen nun noch diejenigen Wirtschaftssubjekte genannt werden, mit denen die obigen Funktionsträger Kontrakte abschließen. Auf den woh: nungsbauunternehmer haben wir schon hingewiesen. Dieser kombiniert die vom Bauherrn erworbenen, für die Gebäudeerstej.lung und -instandhaltung erforderlichen Produktionsfaktoren. $\mathrm{Zu}$ nennen wären zusätzlich noch die Bodeneigentümer bzw. die in deren Interesse und Auftrag handelnden Bodenmakler, die im Besitz der zur Bebauung erforderlichen Grundstücke sind, sowie - auf der Finanzierungsseite - die Kreditgeber; in erster Linie wären dies die Geschäfts- und Hypothekenbanken sowie die Bausparkassen. Be- 
rücksichtigt man darüberhinaus noch die Existenz eines öffentRichen Sektors, der zur Finanzierung seiner Ausgaben Steuern erhebt, so reichen die dargestellten Funktionen aus, um die für uns interessanten Zusammenhänge auf dem Wohnungsmarkt und dem Markt für Bauboden modellartig beschreiben zu können. In Abb.1 wird die Struktur des allgemeinen Modellrahmens grob skizziert.

Betrachtet werden zwei produktive Sektoren - ein "normaler" Unternehmenssektor und der Wohnungssektor -, der Baubodensektor, der Sektor der privaten Haushalte und der öffentliche Sektor. Der Wohnungssektor bezieht vom Unternehmenssektor innerhalb einer Periode Bauleistungen im Umfang von $I_{W}^{b}$ und muB dafür den Geldbetrag $P_{I} I_{W}^{b}$ entrichten, wobei $P_{I}$ für den Preis einer Leistungseinheit steht. Hinter $\mathrm{I}_{\mathrm{W}}^{\mathrm{b}}$ verbirgt sich das gesamte von der Bauindustrie angebotene Güterund Leistungsbündel, also neben den Kapitalgütern auch die für die Errichtung und Instandhaltung benötigte menschliche Arbeitskraft. Um mit Hilfe aller dieser Vorleistungen auch tatsächlich Wohnraum produzieren zu können, benötigt der wohnungssektor Bauboden-im Umfang von F'Flächeneinheiten, die er den Bodeneigentümern zum Preis $\mathrm{P}_{\mathrm{B}}$ je Flächeneinheit abkauft. Die Konsequenz unserer funktionalen Betrachtungs weise ist, daß der Bauboden im unmittelbaren AnschluB an die Bodentransaktion bebaut wird.

In Abb. 1 werden im Rahmen des wohnungssektors auch die für diesen typischen Funktionen genannt; darüberhinaus wird hier schon angedeutet, in welchen steuerlich relevanten Kombinationen die verschiedenen Funktionen auf einzelne Personen verteilt werden können:

- zum einen ist es möglich, daB alle drei Funktionen - die des Bauherrn, des Wohnungsinvestors und des Vermieters von drei verschiedenen personen getragen werden; die durchgezogene Linie (-) kennzeichnet diese Konstellation, die beispielsweise für sogenannte "Bauträgermodelle" typisch ist.

- Der andere Extremfall zeichnet sich dadurch aus, daB alle 
Abb. 1

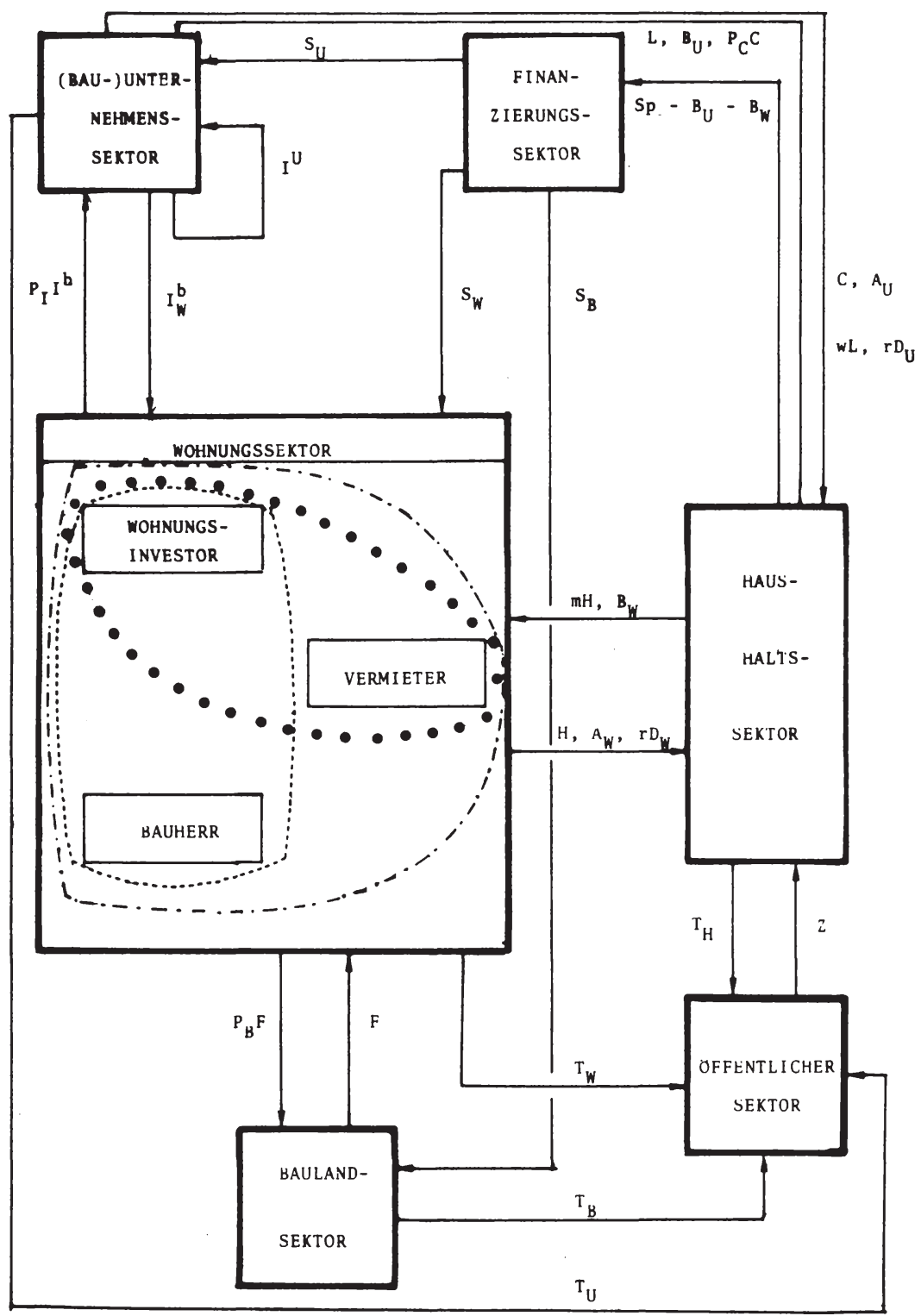


drei Funktionen in ein und derselben person vereinigt sind (-) - Dies entspricht dem Fall des privaten wohnungsinvestors, der seine wohnung selbst errichtet, entweder um sie selbst zu nutzen oder um sie zu vermieten.

- Im Regelfall bei Bauherrenmodellen anzufinden ist die folgende Kombination (---): die Funktionen des Bauherrn und des wohnungsinvestors sind in einer person vereinigt, die des vermieters ist dagegen einer zweiten person übertragen.

- Die gepunktete Linie schließlich beschreibt die situation, in der der Wohnungsinvestor Wohnungseigentum vom Bauträger mit dem ziel der selbstnutzung erwirbt.

wir werden im dritten Kapitel dieser Arbeit sehen, wie unterschiedlich stark die betrachteten drei Funktionen in ihrer Summe der steuerlichen Belastung ausgesetzt sind, abhängig davon, welche der vier eben beschriebenen Kombinationen tatsächlich vorliegt.

Der neu entstandene Wohnraum erhöht ceteris paribus den (annahmegemäß homogenen) Wohnflächenbestand $H$, der zum Preis von $\mathrm{m}$ D-Mark je Flächeneinheit und bezogen auf eine Periode an den Sektor der privaten Haushalte vermietet wird. Implizit ist damit die in der theoretischen Literatur übliche Annahme getroffen, daß jede Wohnflächeneinheit (im Neuzustand) pro Periode genau eine Wohnleistungseinheit produziert.

Neben Wohnleistungen konsumieren die privaten Haushalte zusätzlich ein vom Unternehmenssektor zum Stückpreis von $\mathrm{P}_{C}$ angebotenes Konsumgut in der Menge $C$. Dieses Konsumgut wie auch die vom Wohnungssektor bezogenen Bauleistungen werden mit Hilfe von Kapital und der von den privaten Haushalten zum herrschenden Lohnsatz $w$ angebotenen Arbeitskraft L, gerechnet in Zeiteinheiten, produziert.

Bei der Beschreibung der finanzwirtschaftlichen Sphäre ist es nützlich zu wissen, daß sich jedes Unternehmen letztendlich im Eigentum der privaten Haushalte befindet. Deshalb fließt den Haushalten zumindest ein Teil der Unternehmenserträge in 
Form von Entnahmen oder Ausschüttungen $\mathrm{zu}^{5}$ ! Diese Gewinnanteile werden durch den Parameter A symbolisiert (die Subindizes $W$ und $U$ zeigen $a n$, ob die Entnahmen aus dem "normalen" Unternehmenssektor oder aus dem Wohnungssektor stammen). Andererseits verwenden die privaten Haushalte einen Teil ihrer Ersparnis (Sp) erfahrungsgemä $B$ dazu, die Eigenkapitalbasis ihrer Unternehmen $z u$ erweitern. $B_{W} b z w . B_{U}$ sind die Teile der privaten Ersparnis, die in Form von Beteiligungskapital oder Einlagen an diese Unternehmen zurückfließen. Derjenige Teil der Ersparnis, der nicht unmittelbar für die Zwecke der Kapitalerhöhung Verwendung findet, wird am Kapitalmarkt angeboten. Der Banken- und Sparkassensektor vermittelt dann Kreditkontakte zwischen dem Haushaltssektor einerseits und dem Wohnungssektor sowie dem Unternehmenssektor andererseits. Um unsere Analyse überschaubar zu halten, wollen wir unterstellen, daß sich die Eigentumsrechte an Bauboden bis zum Zeitpunkt des Verkaufs an bauwillige Interessenten nicht verändern und deshalb bei den Bodeneigentumern $\mathrm{bzw}$. den in deren Interesse handelnden Bodenmaklern kein Bedarf an Fremdmitteln entsteht, um etwaige intrasektorale Bodentransaktionen finanzieren zu können.

Bei dem in unserem Modell betrachteten Kapitalmarkt handelt es sich um einen vollkommenen Markt; da zukünftige wie aktuelle Gewinnchancen von allen beteiligten Akteuren korrekt antizipiert werden und eine Beleihung auf der Basis dieser Beträge entsprechend risikolos ist, entstehen für keinen der uns interessierenden Funktionstrăger zu irgend einem zeitpunkt Liquiditätsprobleme. Dies ist sicherlich eine nicht ganz unproblematische Vereinfachung, wenn man die Rolle bedenkt, die eine ausreichende Liquidität für die Zahlungsfähigkeit und damit den Bestand eines Unternehmens spielt. Trotz-

5) Wir wollen im folgenden den Begriff "Entnahmen" als Synonym für beide Formen der Gewinnbeteiligung verwenden. 
dem ist diese Idealisierung vertretbar:

- zum einen wird es dadurch möglich, zwischen den Rentabilitätseffekten und den Liquiditätseffekten bestimmter steuerlicher Regelungen zu unterscheiden. Verfolgt man - um nur ein Beispiel zu nennen - die Diskussion um die im Rahmen von Bauherrenmodellen realisierbaren steuervorteile, so verfestigt sich sehr schnell der Eindruck, daB sich mancher Diskutant der Existenz dieses Unterschieds nicht bewuBt ist.

- Zum anderen spielen Liquiditätsaspekte bei allokationstheoretischen Fragestellungen begründeterweise keine Rolle. Hier geht es allein um die Frage, ob die knappen Ressourcen (bei vorhandener Liquidität) auch tatsächlich in jenen Bereichen eingesetzt werden, die den höchsten Ertrag versprechen.

Der Betrag $S p-B_{U}-B_{W}$ wird in Form von Unternehmensschuldverschreibungen angelegt; dabei erhöht sich der Bestand an Obligationen des Unternehmenssektors $\left(D_{U}\right)$ um den Betrag $S_{U}$ ' der des Wohnungssektors $\left(D_{W}\right)$ um den Betrag $S_{W}$. Beide Sektoren müssen ihre gesamte ausstehende Schuld in jeder Periode zu dem dann gültigen Marktzinssatz $r$ bedienen.

Folglich fließen dem Haushaltssektor in jeder Periode neben den Arbeitseinkommen wL und den Beteiligungsgewinnen $A_{W}+A_{U}$ noch $Z$ inseinkünfte aus schuldverschreibungen in Höhe von $r\left(D_{W}+D_{U}\right) z u$.

Um das periodenbezogene Bruttoeinkommen der privaten Haushalte vollständig beschreiben zu können, müssen schließlich auch noch die staatlichen Transferzahlungen ( $\mathrm{Z}$ ) berücksichtigt werden, die an den Haushaltssektor fließen. Finanziert wird dieser Zahlungsstrom durch Steuerzahlungen $T$, die die Finanzämter im Unternehmenssektor $\left(T_{U}\right)$, im Wohnungssektor $\left(T_{W}\right)$, im Bodensektor $\left(T_{B}\right)$ und im Sektor der privaten Haushalte $\left(T_{H}\right)$ erheben. Welche Steuern dies im einzelnen sind, wird im dritten Kapitel dieser Arbeit beschrieben. 
Damit wäre in groben zügen die struktur der relevanten volkswirtschaftlichen Zusammenhänge umrissen. Um eine intertemporale steuerwirkungsanalyse durchführen zu können, bedarf es noch zusätzlicher Spezifikationen. Um diese Spezifikationen verständlich $\mathrm{zu}$ machen, sind jedoch ejnige grundsätzliche Erläuterungen zum methodischen Vorgehen unerläßlich.

3. Zum methodischen Aufbau der Analyse

Wir werden im folgenden an die Besteuerung des Wohnungs- und des Baulandsektors mit der Forderung herantreten, daß sie die Entwicklung der in Abb.1 strukturierten Modellökonomie hin zu ihrem Wohlfahrtsmaximum nicht behindern darf.

Nun ist mit dieser Forderung noch nicht gesagt, wie die Bedingungen für eine effiziente Allokation der volkswirtschaftlichen Ressourcen im einzelnen lauten. Will man zu einer solchen normativen Aussage gelangen, muß man die Frage beantworten, wie ein allwissender, fast allmächtiger (das Gesetz der knappheit aufheben kann auch er nicht) und vollkommen uneigennütziger Planer handeln würde, wenn man ihm die Verteilung der knappen Ressourcen auf die alternativen Verwendungsmöglichkeiten überließe ${ }^{6}$ ?

Ähnliche Utberlegungen waren wohl auch Ausgangspunkt der Untersuchungen Irving FISHERs ${ }^{7}$, der in seinen Arbeiten Bedingungen für eine optimale intertemporale Allokation ableitete a.llerdings bezogen auf den speziellen Zwei-Perioden-Fall. Nach FISHER stimmen im intertemporalen Marktgleichgewicht die Grenzraten der Gütersubstitution und der Transformation innerhalb einer Periode ebenso überein wie die Grenzrate der Substitution zwischen zukunfts- und Gegenwartskonsum eines Gutes einerseits und die Grenzrate der Transformation dieses Gutes von der einen Periode auf die nächste andererseits. Die Grenz-

6) NACHTKAMP (1982), S.4.

7) $\operatorname{FISHER}(1907,1931)$ 
produktivität des gesamtwirtschaftlichen Kapitalstocks und die Zeitpräferenzrate der privaten Haushalte nehmen dann denselben wert an. Damit erfüllt die optimale Allokation auch im dynamischen Modell des allgemeinen Gleichgewichts die Bedingungen des paretooptimums: keine person, gleichgültig in welcher Periode sie leben mag, kann besser gestellt werden, ohne daß sich die Nutzenposition einer anderen dadurch verschlechtert.

Natürlich ist dieser semi-göttliche Planer eine Fiktion. In den von uns $\mathrm{zu}$ betrachtenden Wirtschaftssystemen werden die Entscheidungen idealtypischerweise nicht zentral gefällt, sondern dezentral auf der Ebene der betroffenen Haushalte und Unternehmen. Wir wissen aber - zumindest lehrt uns dies der zweite Hauptsatz der Wohlfahrtsökonomik - daB sich eben jenes Paretooptimum auch als Konkurrenzgleichgewicht darstellen 1 äßt ${ }^{8}$ ?

Es liegt an dieser Stelle der Einwand nahe, daß Uberlegungen zum Thema "Paretooptimalität" vielleicht von akademischem Interesse sein mögen, daß sie für die praxis aber wenig brauchbare Ergebnisse liefern werden. Denn die Aussage, daß das intertemporale Gleichgewicht einer Konkurrenzökonomie paretooptimal ist, gilt zunächst nur für den Laissez-Faire-Fall, bei dem der staat nicht unmittelbar in das Wirtschaftsgeschehen eingreift. In einer Welt, in der der öffentliche Sektor zum Zwecke der Finanzierung seiner Ausgaben Steuern erhebt, ist dagegen zu befürchten, daß es zu Störungen des Marktprozesses kommt und die daraus resultierenden excess burden eine Abweichung von diesem optimum bewirken.

Wer sich diese Kritik zu eigen macht, übersieht dabei allerdings, daß die Besinnung auf einen ökonomischen Idealtypus durchaus sinnvoll ist - denn nur er versetzt uns in die Lage,

8) Vgl. LANGE (1942); ARROW(1951) lieferte den mathematischen Beweis für die Gültigkeit dieses Satzes;- vgl.auch MALINVAUD(1953), der die Existenz dieses intertemporalen Gleichgewichtszustandes aligemeir für ein Entscheidungsproblem mit unendlichem Planungshorizont beweist; ebenso DORFMAN/SAMUEISON/SOLOW(1958), insbes. S.310 ff. 
praktikabie und praktizierte Problemlösungen nicht nur beschreiben, sondern auch unter normativen Gesichtspunkten bewerten $\mathrm{zu}$ können.

Die paretooptimale Allokation der (hypothetischen) LaisserFaire-Konkurrenzökonomie liefert uns den Maßstab für einen wirtschaftlichen Ebfizienztest, der es uns ermöglicht, real existierende steuerliche Regelungen auf ihren allokationstheoretischen Sinngehalt zu überprüfen.

Damit wäre der strategische Grundgedanke dieser Arbeit auch schon beschrieben; die analytische Konzeption ist demnach zweistufig. Auf der ersten stufe werden, ausgehend von der Laisser-Faire-Situation, die Bedingungen für ein Paretooptimum abgeleitet. Auf der zweiten Stufe werden die spezifischen, den Wohnungssektor betreffenden steuerlichen Vorschriften implementiert und untersucht, ob und in welcher Weise diese Besteuerung verzerrend wirkt - sprich: ob und in welcher Weise die auf Stufe $\mathrm{Zwei}$ abgeleiteten Bedingungen von denen der Stufe Eins abweichen - und welche Modifikationen des aktuellen Steuerrechts gegebenenfalls vorzunehmen wären, um dessen allokative Neutralität zu sichern.

Auf den ersten Blick scheint es schwierig, wenn nicht gar unmöglich zu sein, im Rahmen des in Abb. 1 skizzierten Modells Bedingungen für ein allgemeines intertemporales Konkurrenzgleichgewicht abzuleiten - müssen doch offensichtlich nicht weniger als vier intertemporale Entscheidungsprobleme simultan gelöst werden. Aber auch aus diesen Schwierigkeiten vermag uns die FISHERsche Theorie herauszuhelfen. Wie diese Hilfestellung im einzelnen aussieht, läßt sich am einfachsten anhand eines graphischen Beispiels demonstrieren ${ }^{9}$ ?

Betrachten wir hierzu einen Jungbauer, der von seinem Vater als einziges einen Sack Weizen geerbt hat. Er steht nun vor der Aiternative, den gesamten Weizen in der jetzigen Periode zu konsumieren oder einen Teil davon aufzusparen und

9) HIRSHLEIFER(1974), S.31 ff. 
auszusäen, um auch in der nächsten Periode noch etwas zum Leben zu haben. Zwischen den heute ausgesäten und den morgen zu erntenden Weizenkörnern existiert eine funktionale Beziehung der Form

$$
\text { (I.1) } \quad C^{2}=f(I), f^{\prime}>0, f^{\prime}{ }^{\prime}<0 \text {, }
$$

mit

$$
\text { (I.2) } \quad I=W-C^{1} \text {, }
$$

wobei $\mathrm{C}^{2}$ für die $\mathrm{zahl}$ der Weizenkörner steht, die morgen geerntet und konsumiert werden können, wenn heute I Körner ausgesät werden. $W$ symbolisiert die Menge der geerbten und $c^{1}$ die Menge der heute verspeisten Weizenkörner. In Abb.2 gibt die Transformationskurve WW die in (I.1) beschriebenen Produktions- und Konsummöglichkeiten wieder.

$\mathrm{Abb} .2$

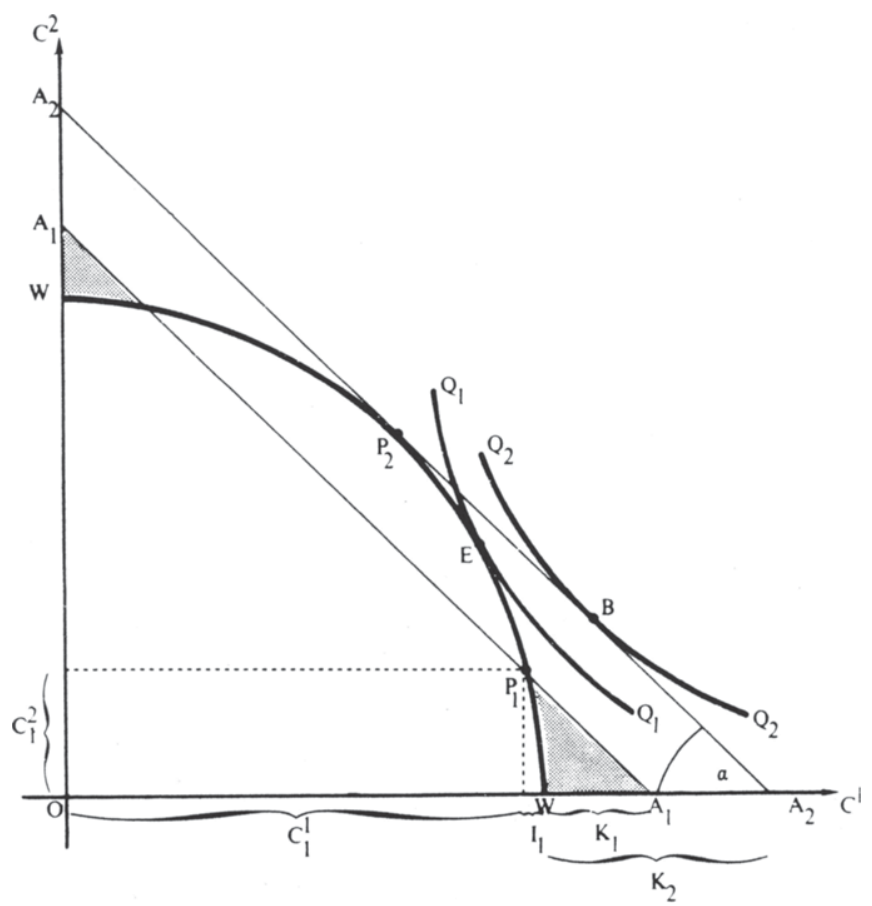


Dem Bauer bietet sich nun aber zusätzlich die Möglichkeit, seinen Konsum der ersten Periode dadurch zu erhöhen, daß er sich, ausgehend von einer gegebenen Produktionsentscheidung, auf dem Kreditwege zusätzliches Getreide verschafft, wofür er aber Anspruiche auf seine Ernte in Periode 2 an den Kreditgeber abtreten muß; diese Ansprüche umfassen nicht nur den ursprünglichen Kreditbetrag - den wir einmal D nennen wollen -, sondern zusätzlich noch die $\mathrm{Zinsen}$ auf diesen Kredit, die ihrerseits die Höhe $r D$ annehrien, wenn $r$ der $\mathrm{Z}$ ins für solche intertemporalen Tauschkontrakte ist. Umgekehrt kann er natürlich auch Kredite vergeben: verzichtet er in der ersten Periode auf den Konsum von D Getreidekörnern und verleiht er diese, so erhöht sich dadurch sein Konsumpotential der zweiten Periode um $(1+r) D$ auf $C_{2}+(1+r) D$ Getreidekörner.

offensichtlich vermehrt die Möglichkeit, intertemporale Tauschkontrakte abschließen und auf diesem Wege Zukunftskonsum in Gegenwartskonsum bzw. Gegenwartskonsum in Zukunftskonsum transformieren zu können, die Konsumalternativen des Bauern. Die Markttransformationsgeraden $A_{1} A_{1}$ und $A_{2} A_{2}$, die beide die Steigung $-(1+r)$ besitzen, spiegeln diesen Sachverhalt graphisch wider: wählt der Bauer den Produktionspunkt $\mathrm{P}_{1}$, so erweitert alleine die Existenz eines Marktes für intertemporale Tauschkontrakte den Konsummöglichkeitsbereich um die schraffierten Flächen in Abb.2.

Die Wahl des Produktionspunktes $\mathrm{P}_{1}$ wäre aber unklug; da es letztendlich das ziel des Bauern sein muß, seine potentiellen Konsummengen zu maximieren - unabhängig davon, wie die Konsumentscheidung dann tatsächlich ausfällt -, kann der optimale Produktionsplan nur $\mathrm{P}_{2}$ sein.

Der Vorteil, den die Existenz eines Kapitalmarktes - dies ist der Markt, auf dem intertemporale Tauschkontakte abgeschlossen werden - bietet, läßt sich auch bewerten: gerechnet in Gegenwartsgrößen und Getreidekörnern ist dies - unter der Voraussetzung, daß der optimale Produktionsplan gewählt wurde - die Differenz $z$ wischen dem maximal möglichen Gegenwartskonsum $\mathrm{OA}_{2}$ 
und der Anfangsausstattung OW. Diese Differenz entspricht dem Kapitalwert des bäuerlichen Betriebes; $\mathrm{OA}_{2}$ kann man dementsprechend als dessen Ertragswert oder Marktwert bezelchnen.

Der Bauer kann sich nun aus der Menge von Konsumalternativen jene auswählen, welche seinen individuellen Nutzen maxi-. miert. Unter den üblichen Annahmen für die Nutzenfunktion (strikte Quasikonkavität, streng positiver Grenznutzen) wird ein Konsumpunkt auf der Kapitalmarktgeraden $\mathrm{A}_{2} \mathrm{~A}_{2}$ gewählt; in $\mathrm{Abb} .2$ ist dies der Punkt B. Hätten sich dagegen keine Tauschmöglichkeiten eröffnet - gäbe es keinen Kapitalmarkt -, wäre E der nutzenmaximale Konsumplan gewesen; dieser Punkt liegt auf einem eindeutig niedrigeren Indifferenzniveau als B.

Es ist offensichtlich, daB in dem in Abb. 2 beschriebenen intertemporalen Gleichgewicht die Grenzproduktivität des Produktionsfaktors Weizen übereinstimmt mit der subjektiven Zeitpräferenzrate des bäuerlichen Haushalts ${ }^{10}$ ?

Wichtiger als dieses Ergebnis sind für unsere weitere Analyse die methodologischen Konsequenzen, die die Existenz eines Kapitalmarktes impliziert:

Die Produktionsplanungen der unternehmen und die Konsumplanungen der privaten Haushalte brauchen nicht simultan analysiert zu werden. Wie gezeigt wurde, ist die produktionsentscheidung des Bauern unabhängig von seiner Präferenzstruktur.

Dies ist die Kernaussage des in der Literatur so bezeichneten Trennungstheorems, welches uns erlaubt, die Entscheidungsprobleme der beiden produktiven Sektoren und des Bodensektors -

10) Da die Markttransformationsgerade $A_{2} A_{2}$ im Gleichgewicht sowohl die Transformationskurve iW als auch die Indifferenzkurve $Q_{2} Q_{2}$ tangiert, gilt bzw. Grenzrate der Transformation $=-(1+r)=$ Grenzrate der Substitution, bzw.

|Grenzrate der Transformation - $1|=r=|$ Grenzrate der Substitution - 1|, Grenzproduktivität des Weizens $=r=$ Zeitpräferenzrate des Haushalts. 
und auch hier wiederum jedes für sich - auf der einen seite und des Haushaltssektors auf der anderen seite isoliert $\mathrm{zu}$ betrachten.

Die Implikationen des Trennungstheorems wollen wir unserem weiteren Analyseweg dienstbar machen. Wir werden im folgenden eine Partialanalyse betreiben und unser Hauptaugenmerk hierbei auf den Wohnungssektor und den Baulandsektor richten, ohne allerdings die Verzahnung dieser beiden Sektoren mit den restlichen Bestandteilen der in $\mathrm{Abb} .1$ beschriebenen Modellökonomie aus den Augen zu verlieren. Wir gehen hierbei von der Annahme aus, daB es sich bei den betrachteten Wohnungsunternehmern und Bodenmaklern um vollkommene Konkurrenten handelt und unterstellen zusätzlich, daß sich jeder sektor anhand eines typischen vertreters beschreiben läßt.

Die beiden repräsentativen Unternehmen befinden sich im Eigentum des Haushaltssektors, auch er wiederum durch einen typischen Repräsentanten vertreten. Alle Aktivitäten der beiden unternehmerischen Akteure müssen folglich darauf ausgerichtet sein, den aus einem festzulegenden intertemporalen Konsumplan resultierenden Nutzen des Haushalts zu maximieren. Und Abb. 2 sagt uns, mit welcher zielsetzung der Wohnungsinvestor seinen Wohnungsbestand und der Bodenmakler den Zeitpfad seiner Baulandverkäufe planen muß, um dieser Anforderung zu genügen:

Die planungen der beiden Akteure sind dann und nur dann die optimalen, wenn die Kapitalwerte bzw. die Marktwerte beider unternehmen dadurch maximiert werden.

Dann (und nur dann) ist nämlich auch der Konsummöglichkeitsbereich des repräsentativen Eignerhaushalts der größtmögliche.

Dementsprechend werden wir unsere folgenden Uberlegungen zwei repräsentativen Unternehmern widmen, die ihr Verhalten aus intertemporalen mikroökonomischen optimierungsansätzen ableiten. Wohnungsinvestor und Bodenmakler treffen ihre Entscheidungen unabhängig voneinander, eine Abstimmung der Pläne findet nicht statt. Diese Koordinationsfunktion wird von den 
Märkten übernommen. Beide Akteure verhalten sich als Mengenanpasser; sie nehmen die Pfade der Marktpreise bei ihren Planungen als exogen gegeben hin. Gleichwohl sind die Marktpreispfade, bezogen auf unseren Modellrahmen, endogen - sie sind das Ergebnis des Aufeinandertreffens von Angebot und Nachfrage auf den betreffenden Märkten. Da beide Akteure zudem über perfekte Voraussicht verfügen, spielen sich die Preispfade so ein, daß beider Pläne $z u$ jedem Zeitpunkt miteinander kompatibel sind. Deshalb hat auch keiner von beiden das Bedürfnis, eine in der Vergangenheit getroffene Entscheidung jemals zu revidieren. 
II .

DIE LAISSEZ-FAIRE- OKONOMIE UND DIE BEDINGUNGEN FUR EINE EFFIZIENTE ALLOKATION DES GEBÄUDEKAPITALS UND DES BAULANDES

Ziel dieses Kapitels ist es, Bedingungen abzuleiten, die der Charakterisierung des Allokationsoptimums dienen. Diese Bedingungen sind identisch mit jenen Maximen, gemäß denen in einer steuerfreien Welt Wohnungsunternehmer ihre Investitionen und Bodenmakler ihr Baulandangebot planen würden, um dem ziel der Maximierung des jeweiligen Unternehmensmarktwertes zu genügen.

Wir starten unsere Analyse zu einem zeitpunkt $t_{0^{\prime}} z u$ dem durch eine übergeordnete Planungsbehörde ein Bodenareal im Umfang von

(II. 1)

$$
B\left(t_{0}\right)>0
$$

homogenen Flächeneinheiten zur Bebauung freigegeben wird. Diese Anordnung sieht ebenfalls vor, daß noch nicht bebautes Bauland einer anderen Nutzungsform nicht zugeführt werden darf, sondern brachzuliegen hat.

Ebenso sei zum Ausgangszeitpunkt $t_{0}$ aufgrund früherer Bautätigkeit ein homogener Wohnflächenbestand

$$
H\left(t_{0}\right)>0
$$

vorhanden.

1. Das Planungsproblem des Wohnungsinvestors

Zu Anfang des Planungsproblems verfügt der Wohnungsinvestor somit über einen Wohnflächenbestand von $H\left(t_{0}\right)$ Flächeneinheiten, der durch weitere Investitionen aufgestockt werden kann. Ferner existieren in $t_{0}$ aufgrund in der Vergangenheit getroffener Finanzierungsentscheidungen Verbindlichkeiten des Wohnungsinvestors gegenüber dem kreditsektor in Höhe von

$$
D\left(t_{0}\right) \lesseqgtr 0 \text {. }
$$


Neuen Wohnraum kann der Wohnungsinvestor schaffen, indem er sich auf einem Baugrundstück von $F^{d}$ Flächeneinheiten von dem modellexogenen Unternehmen ein schlüsselfertiges Gebäude errichten läßt. Das Grundstück hat er zuvor zum - von ihm als Datum akzeptierten - Preis $P_{B}$ je Flächeneinheit von dem repräsentativen Bodenmakler erworben; das Wohnungsbauunternehmen läßt sich die von ihm bereitgestellten Bauleistungen zum Preis von $P_{I}$ je Leistungseinheit honorieren.

Zwischen dem neugeschaffenen Wohnraum einerseits und den Inputs Boden $\left(F^{d}\right)$ sowie Neubauleistungen (I) andererseits besteht eine funktionale Beziehung der Form

$$
h=f\left(I, F^{d}\right) \text {. }
$$

Wir wollen für die Zwecke unserer Analyse die in der Produktionstheorie üblichen Annahmen treffen, daß $f$

- strikt quasi-konkav und

- linear homogen ist und

- über eine Substitutionselastizität $\sigma=-1$ verfügt. In diesem Falle läßt sich (II.4) normieren zu

$$
h=F_{f}^{d}\left(\frac{I}{F}, 1\right)=F^{d} \varphi(\varepsilon) .
$$

Die Variable $\varepsilon$ steht in (II.5') für den Quotienten aus Bauleistungseinsatz und Bodenverbrauch. Sie wird im folgenden als Kapitalintensität der Bebauung bezeichnet; $\varphi(\varepsilon)$ gibt folgerichtig die neuproduzierte Wohnfläche pro Bodeneinheit wieder.

Wir wollen darüberhinaus unterstellen, daß es sich bei (II.4) um eine Produktionsfunktion des putty-clay-Typs handelt; diese Annahme spiegelt die bei der Produktion von Wohnflächeneinheiten herrschenden technischen Gegebenheiten - man denke nur an die Stahlbetonbauweise - wohl am realistischsten wider: $z u$ dem Zeitpunkt, zu dem Bauland und Bauleistungen miteinander komhi- 
niert werden, verlieren sie ihre spezifischen Eigenschaften: der Produktionsprozess bei der Gebäudeerrichtung ist nicht umkehrbar. Um die Kapitalintensität $\varepsilon$ des Grundstücks im nachhinein $z u$ verändern, ist es deshalb erforderlich, das aufstehende Gebäude zuerst abzureißen, um danach ein neues, größeres Gebäude errichten zu können. Aus Gründen der rechentechnischen Vereinfachung wollen wir annehmen, daß die Abrißkosten so hoch sind, daß es sich zu keinem zeitpunkt lohnt, ein einmal errichtetes Gebäude wieder abzureißen.

Unter diesen Umständen wird sich der Wohnungsinvestor sehr genau überlegen müssen, wieviel Bauland er heute mit welcher Intensität bebauen (lassen) soll, ja sogar, ob er mit der Bebauung eines Grundstücks nicht besser warten sollte, um einer in der zukunft höheren Wohnleistungsnachfrage der privaten Haushalte dadurch gerecht werden zu können, daß er auf demselben Grundstück zu einem späteren Zeitpunkt ein größeres Gebäude errichtet. Dieses Entscheidungsproblem läßt sich anhand eines einfachen Beispiels darstellen:

Stellen wir uns zum Zeitpunkt $t_{0}$ einen Wohnungsinvestor vor, der die Absicht hat, ein von ihm noch $\mathrm{zu}$ erwerbendes Grundstück $z u$ bebauen. Probleme bei seiner Investitionsentscheidung bereitet ihm das Wissen, daß sich die Nachfrage nach Wohnleistungen in den nächsten $\left(t_{1}-t_{0}\right)$ Perioden nicht verändert, sich zum zeitpunkt $t_{1}$ sprunghaft erhöht und jenseits von $t_{1}$ dauerhaft auf diesem höheren Niveau verharrt. Angesichts dieser Entwicklung steht der Wohnungsinvestor vor drei Entscheidungsalternativen; er kann

- heute auf dem Grundstück ein kleines Haus errichten, das der Nachfragesituation der Zeitspanne von $t_{0}$ bis $t_{1}$ Rechnung trägt;

- heute mit Blick auf die zukünftige Nachfrageentwicklung auf demselben Grundstück ein im Vergleich zur ersten Alternative größeres Haus errichten;

- mit dem Bau des größeren Gebäudes bis zum Eintritt der Nachfrageänderung in $t_{1}$ warten. 
Jede dieser drei Alternativen ist aus der Sicht des Wohnungsinvestors mit spezifischen Vor- und Nachteilen verbunden:

- die erste Alternative bietet den Vorteil, daß das Haus bis zum zeitpunkt $t_{1}$ "optimal" genutzt werden kann; ihr Nachteil besteht darin, daß der Wohnungsinvestor, falls er sich für diese Alternative entscheidet, jenseits von $t_{1}$ dauerhaft auf Mieterträge verzichtet, in deren Genuß er durch den Bau eines größeren Hauses hätte kommen können;

- die Vor- und Nachteile der zweiten Alternative verhalten sich zu denen der ersten genau entgegengesetzt; errichtet der Wohnungsinvestor in $t_{0}$ ein Haus, das mit Blick auf die nach $t_{1}$ herrschenden Nachfrageverhältnisse konzipiert wurde, so riskiert er, daß er Teile dieses Gebäudes bis $t_{1}$ nicht vermieten kann. Hätte er das in die leerstehenden Wohnungen investierte Vermögen stattdessen am Kapitalmarkt angelegt, hätte er $\mathrm{Z}$ inseinkünfte realisieren können. Diesen im Vergleich zur ersten Alternative höheren opportunitätskosten steht jedoch der Vorteil höherer Mieteinnahmen gegenüber, die sich jenseits von $t_{1}$ durch die Vermietung des größeren Hauses erzielen lassen;

- bei der dritten Alternative verzichtet der Wohnungsinvestor in der Phase $\left(t_{0}, t_{1}\right)$ ganz auf Mieteinnahmen. Dieser im Vergleich zu den Alternativen Eins und Zwei offensichtliche Nachteil wird dadurch gemildert, daB die sonst in den Wohnungsbau investierten Geldbeträge am Kapitalmarkt angelegt werden und dort $\mathrm{zinserträge} \mathrm{erwirtschaften} \mathrm{können.} \mathrm{Zudem} \mathrm{ent-}$ stehen dem Wohnungsinvestor, falls er für diese dritte Alternative optiert, anders als bei der Variante $\mathrm{Zwei}$ keine opportunitätskosten infolge von Leerständen. Der komparative Vorteil gegenüber Alternative Eins besteht darin, daß zum Zeitpunkt $t_{1}$ ein Gebäude errichtet wird, das speziell auf die nach $t_{1}$ herrschenden Nachfrageverhältnisse zugeschnitten ist und entsprechend höhere Mieterträge verspricht. 
Welcher dieser drei Alternativen der Wohnungsinvestor den Vorzug gibt, läßt sich a priori nicht sagen - im Einzelfall werden die Höhe des Kapitalmarktzinses, die Länge der zeitspanne $\left(t_{1}-t_{0}\right)$, das Niveau der Wohnungsmiete sowie das Ausmaß der Nachfrageänderung nach Wohnleistungen ausschlaggebend sein für die wahl einer dieser drei Varianten.

Eines läßt sich jedoch mit sicherheit sagen:

Die wahrscheinlichkeit, daß sich der wohnungsinvestor für die dritte Alternative entscheidet und den Bebauungszeitpunkt von $t_{0}$ auf $t_{1}$ verschiebt, ist ceteris paribus umso höher, je gröBer zum Zeitpunkt $t_{1}$ die Änderung der Nachfrage nach wohnleistungen ausfällt.

Je ausgeprägter nämlich dieser Nachfrageschub ist, desto schwerer fallen di.e komparativen Nachteile der Alternativen Eins und Zwei ins Gewicht: wird in Periode $t_{0}$ ein kleines Haus gebaut, nimmt der in der Phase $\left(t_{1}, \infty\right)$ eintretende Verzicht auf mögliche Mieteinnahmen bei einer starken Nachfrageänderung ein für den Wohnungsinvestor u.U. nicht mehr akzeptables Ausmaß an. Das gleiche gilt für den Mietausfall in der Phase $\left(t_{0}, t_{1}\right)$, wenn der Wohnungsinvestor für Variante $\mathrm{zwei}$ optiert und in $t_{0}$ ein Haus errichtet, das auf die Nachfrageverhältnisse jenseits von $t_{1}$ - für die Zeitspanne $\left(t_{0}, t_{1}\right)$ also viel zu groß - abgestimmt ist.

Die Intuition für diese Planungsprobleme ist für das ökonomische Verständnis der im folgenden noch $\mathrm{zu}$ beschreibenden Modelldynamik von zentraler Bedeutung.

Die Annahme der Irreversibilität der Bebauung wird tendenziell dadurch aufgeweicht, daß das Gebäudekapital im Zeitablauf abnutzungsbedingt an Substanz verliert. Wenn wir die in der Kapitaltheorie übliche Annahme treffen, daß das (Wohnungs-) Kapital mit einer konstanten Rate $\delta$ von Periode zu Periode stetig schrumpft ${ }^{12}$ ), so existieren von einer $\mathrm{zu}$ einem

12) Vgl. JORGENSON (1967) 
beliebigen Zeitpunkt $t<t_{0}$ getätigten Neubauinvestition zum Zeitpunkt $t_{0}$ gerade noch

(II. 6) $\quad h^{t}\left(t_{0}\right)=\exp \left[-\delta \alpha\left(t_{0}-t\right)\right] F^{d}(t) \varphi[\varepsilon(t)], 0<\alpha<1, \delta>0$

Wohnflächeneinheiten; in (II.6) beschreibt a die partielle

Produktionselastizität des Gebäudekapitals ${ }^{13}$ )

Mit Hilfe von (II.6) und unter Vernachlässigung werterhaltender Maßnahmen läßt sich dann der gesamte zum Zeitpunkt $t_{0}$ verfügbare Wohnflächenbestand $\mathrm{H}$ wie folgt beschreiben:

$$
H\left(t_{0}\right)=\int_{-\infty}^{t_{0}} \exp \left[-\delta \alpha\left(t_{0}-t\right)\right] F^{d}(t) \varphi[\varepsilon(t)] d t .
$$

Um zu sehen, wie sich H unter dem Einfluß von Wohnungsneubau einerseits und abnutzungsbedingter Substanzminderung andererseits verändert, muß man (II.7) nach dem Zeitindex $t$ differenzieren. Für $t=t_{0}$ erhält man dann

$$
\frac{\dot{H}\left(t_{0}\right)}{\partial t_{0}}=\dot{H}\left(t_{0}\right)=F^{d}\left(t_{0}\right) \varphi\left[\varepsilon\left(t_{0}\right)\right]-\alpha \delta H\left(t_{0}\right) \cdot
$$

Die periodenbezogene Veränderung des Wohnflächenbestandes stellt sich als eine NettogröBe dar; sie entspricht der Differenz aus dem in der betrachteten Periode neu

13) (II.6) wird verständlicher, wenn man bedenkt, daß die oben getroffenen Annahmen eine Produktionsfunktion des Cobb-Douglas-Typs implizieren; es gilt dann

$$
h(t)=I(t) \alpha_{F(t)}(1-a) \text {. }
$$

Betrachtet man dasselbe bebaute Grundstück ( $t-t)$ Perioden später, so existieren infolge der Abnutzung des Gebäudekâpitals gerade noch

$$
\begin{aligned}
h^{t}\left(t_{0}\right) & =\left[I(t) e^{-\delta\left(t_{0}-t\right)}\right]^{\alpha_{F}(t)}(1-\alpha) \\
& =e^{-\delta \alpha\left(t_{0}-t\right)} F \varepsilon^{\alpha}
\end{aligned}
$$

Wohnflächeneinheiten.

14) Wir wollen für den Rest der Arbeit die folgenden Konventionen treffen: für die Ableitung einer Variablen $x$ nach der Zeit $t$ gilt $d x / d t \pm \dot{x}$, für die relative veränderung dieser variablen im Zeitablauf gilt $\dot{\mathrm{X}} / \mathrm{x} \equiv \hat{\mathrm{X}}$. 
errichteten Wohnraum einerseits und den via Abnutzung aus dem Bestand ausgeschiedenen Wohnflächeneinheiten andererseits. $\mathrm{Da} \beta$ die auf den Wohnflächenbestand bezogene Abschreibungsrate nicht identisch ist mit $\delta$, sondern mit dem Produkt aus $\delta$ und der partiellen Produktionselastizität a des Gebäudekapitals ist die unmittelbare Folge der Tatsach€:, daß nur das Gebäuclekapital, nicht aber auch das Bauland der Abnutzung unterliegt.

Keine Berücksichtigung findet in unserer Analyse das in der Praxis oft $z \mathrm{u}$ beobachtende Phänomen, daß der Wohnungsinvestor die periodenbedingte Substanzminderung seiner Wohnimmobilie durch eine entsprechende Wahl der Intensität der Wohnungsnutzung beeinflussen kann. So interessant diese Problematik für Fragen ist, die sich im Zusammenhang mit dem zielkonflikt zwischen optimalem Wohnungsmanagement auf der einen Seite und sozialen und humanitären Aspekten des Wohnens auf der anderen Seite stellen, so wenig Interesse vermag sie im Hinblick auf die von uns $\mathrm{zu}$ behandelnden Fragestellungen $\mathrm{zu}$ wecken.

In (II.8) ebenfal.ls nicht berücksichtigt ist die Tatsache, daß der Wohnungsinvestor grundsätzlich immer die Möglichkeit hat, den abnutzungsbedingten Substanzverlust mittels werterhaltender Maßnahmen aufzuholen oder durch entsprechende Modernisierungsarbeiten sogar noch zu übertreffen. Die Differenzierung nach Erhaltungsaufwand und Modernisierungsaufwand empfiehlt sich hierbei nicht nur aufgrund semantischer Erwägungen: bei Erhaltungsaufwand handelt es sich im Prinzip um Reinvestitionen, für die - bei gegebenen Preisen - dieselbe Kostenfunktion gilt wie für die Erstinvestition. Wenn z.B. in einem Altbau die alte Heizungsanlage durch eine neue, aber artgleiche Heizungsanlage ersetzt wird, so werden unter den getroffenen Annahmen die Kosten für den Kauf und den Einbau der neuen Anlage mit denen der alten Anlage übereinstimmen. Wird dagegen anstelle der artgleichen eine Anlage mit neuer Technik (z.B. eine, die aus einem Fernwärmenetz gespeist wird) installiert, so müssen in aller Regel zusätzlich zu den Ein- 
kaufs- und Installationskosten noch Kosten für konstruktive Änderungen am Gebäude getragen werden. Die Kostenstruktur für Modernisierungsaufwand unterscheidet sich somit grundsätzlich von der des Erhaltungsaufwandes.

Während die Berücksichtigung von werterhaltenden Maßnahmen aus den oben genannten Gründen unproblematisch ist, würde die Einführung einer separaten Kostenfunktion für Modernisierungsaufwand die Analyse erheblich komplizieren, ohne uns aber $z u$ grundlegend neuen Erkenntnissen verhelfen $z u$ können. Aus diesem Grunde ist es sicherlich vertretbar, Modernisierungsaufwendungen aus unserer Analyse auszuklammern.15)

Für den Wohnungsinvestor hat diese Vereinfachung zur Konsequenz, $d a \beta$ er eine Erweiterung seines Wohnflächenbestandes nur auf dem Weg des Wohnungsneubaus vornehmen kann. Mit Hilfe werterhaltender Maßnahmen lassen sich lediglich die abnutzungsbedingten Substanzminderungen des Gebäudekapitalbestandes kompensieren. Bezeichnen wir den Umfang an werterhaltenden Maßnahmen in einer Periode $t$ mit dem Parameter $E(t)$ (infolge der Irreversibilität der Bebauung gilt $\left.E(t) \geq 0, t_{0} \leq t \leq \infty\right)$, so mu für $E(t)$ als obere Schranke folgerichtig

$$
E(t) \leq \alpha \delta H(t) \text { für } H(t)=H(t)
$$

gefordert werden. $H(t)$ bezeichnet hierbei denjenigen bis zur Periode $t$ akkumulierten Wohnflächenbestand, der sich ergeben hätte, wenn entweder das Gebäudekapital nicht der Abnutzung unterlegen hätte oder in jeder Periode sämtliche Substanzverluste

15) Ein alternativer Weg, dieses Problem analytisch zu lösen, bestünde darin, für den Modernisierungsaufwand dieselbe Kostenfunktion wie für werterhaltende Maßnahmen und Neubauinvestitionen zu unterstellen. Damit würäe aber die Annahme der Irreversibilität der Bebauung quasi durch die Hintertür wieder aufgehoben: Bestandsanpassungen wären zu jedem Zeitpunkt und in beliebigem Umfang möglich, ohne daß dazu zusätzlicher Baugrund erforderlich wäre. 
durch entsprechende Erhaltungsarbeiten kompensiert worden wären. $H(t)$ steht dagegen für den tatsächlich zum zeitpunkt $t$ vorhandenen Wohnflächenbestand - er als das Resultat des bis zum Zeitpunkt $t$ tatsächlich realisierten Neubau- und Renovierungsprogramms. Bedingung (II.9) stellt sicher, daß es mit Hilfe werterhaltender Maßnahmen zwar möglich ist, jedes zum zeitpunkt $t$ vorhandene Gebäude in exakt jenen Zustand $z u$ versetzen, den es zum Zeitpunkt seiner Fertigstellung innehatte, darüberhinausgehende Erhöhungen des Wohnflächenbestandes aber nur auf dem Wege des Wohnungsrieubaus vorgenommen werden können ${ }^{16)}$

Dementsprechend müssen wir die Bewegungsgleichung des Wohnflächenbestandes aus (II.8) um den Tatbestand des Erhaltungsaufwandes erweitern; wir erhalten unter Beachtung von (II.9) als modifizierte Bewegungsgleichung für den Wohnflächenbestand die Beziehung

$$
\dot{H}\left(t_{0}\right)=F^{d}\left(t_{0}\right) \varphi\left[\varepsilon\left(t_{0}\right)\right]-\left[\alpha \delta H\left(t_{0}\right)-E\left(t_{0}\right)\right] .
$$

Um den Produktionsmittelcharakter der Wohnung eindeutig herauszustellen, wird angenommen, daß jede $\mathrm{zu}$ einem beliebigen Zeitpunkt $t$ vorhandene Wohnflächeneinheit zu diesem Zeitpunkt genau eine Wohnleistungseinheit abgibt; folglich steht $H(t)$ nicht nur für den Wohnflächenbestand, sondern auch für den Strom der zum Zeitpunkt $t$ produzierten Wohnleistungseinheiten. Diese werden nun zum Preis von $m$ D-Mark pro Einheit und Periode an den repräsentativen Haushalt verkauft; $m$ steht hierbei für

16) KAMIEN/SCHWARTZ (1981), S.215 ff. 
die Mietgrenzzahlungsbereitschaft des Haushalts. Diese wird zum einen vom dem Angebot $\mathrm{H}$ an Wohnungsnutzungseinheiten einer gegebenen Periode abhängen, zum anderen aber auch von der vom repräsentativen Haushalt bei alternativen Mietpreisen entfalteten Nachfrage, die wir mit dem Parameter a bezeichnen wollen. Dte in der mikroökonomischen Analyse üblicherweise getroffene Annahme, daß der Preis mit wachsendem Angebot und sinkender Nachfrage fält (et vice versa), wollen wir auch in unserer Analyse verwenden; unterstellt wird der funktionale zusammenhang

$$
m(t)=m\left[\frac{a(t)}{H(t)}\right], m_{H}<0, m_{a}>0,
$$

mit einer konstanten absoluten Preiselastizität der Wohnleistungsnachfrage

$$
n=\left|\frac{m H}{m^{\prime} a}\right|=\text { konstant }>0 \text {. }
$$

Es ist nicht zu erwarten, daß der Parameter a im zeitablauf konstant bleibt. Vielmehr muß aufgrund der demographischen Entwicklung mit einem stetigen Anstieg der Wohnleistungsnachfrage gerechnet werden. Auch die Tatsache, daß im Zuge des gesamtwirtschaftlichen Akkumulationsprozesses das individuelle Vermögen stetig wächst, wird sich im Zweifelsfalle in einer Erhöhung des pro-Kopf-Wohnkonsums niederschlagen. Wir wollen deshalb annehmen, daß die Nachfrage nach Wohnleistungen von Periode zu Periode mit einer konstanten Rate zunimmt:

$$
\text { (II.13) } \quad \hat{a}=\text { konstant }>0 \text {. }
$$

Graphisch läBt sich dieser Zusammenhang wie folgt darstellen: trägt man in einem Preis-Mengen-Diagramm die nachgefragte Menge an Wohnleistungen als fallende Funktion des Mietzinses $a b$ (wie in Abb.3 geschehen), so verschiebt sich die Nachfragekurve unter dem EinfluB der permanent wachsenden 
Nachfrage nach Wohnkonsum und sonst gleichen Bedingungen stetig nach rechts.

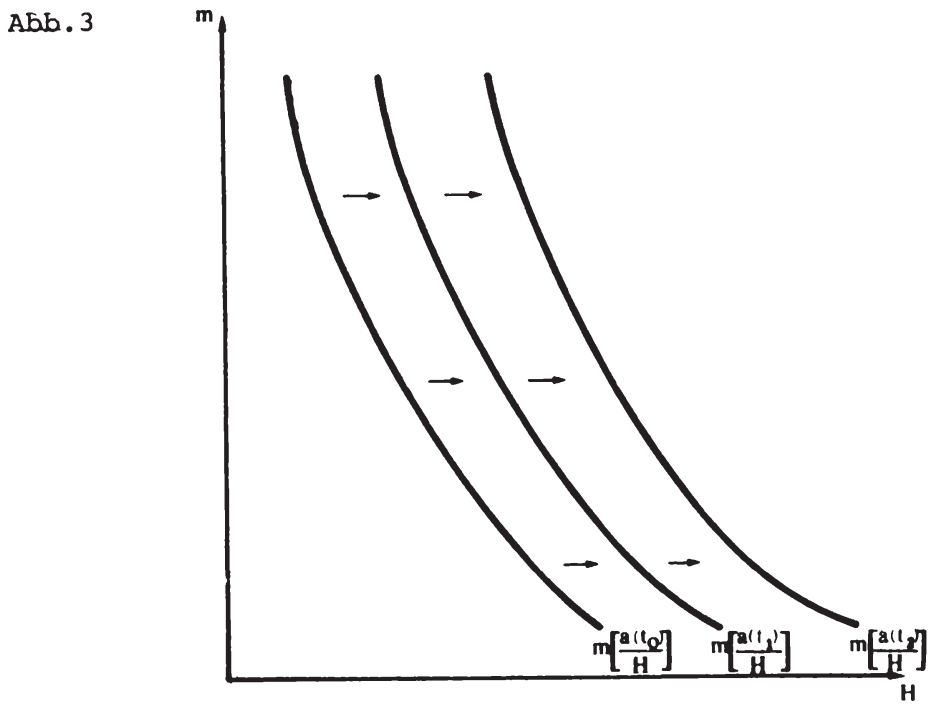

Der Wohnungsinvestor erzielt in jeder beliebigen Periode $t$ Bruttomieteinnahmen in Höhe von $m(t) H(t)$ D-Mark. Dieser Gesamtbetrag steht jedoch nicht in voller Höhe für Entnahmezwecke zur Verfügung. Den entnahmefähigen cash flow des Wohnungsunternehmens erhalten wir, wenn wir von den Bruttomieteinnahmen die Ausgaben für Bauleistungen, für Bauland, für den Zinsendienst und für die Tilgungsleistungen abzüglich der neu geschlossenen Kreditkontrakte subtrahieren. Die beiden letzten Positionen lassen sich saldiert unter dem stichwort "Nettoneuverschuldung" zusammenfassen, für die wir das symbol s verwenden wollen. Für positive Werte von $s$ übertrifft die Neuverschuldung die Tilgungsleistungen des Wohnungsinvestors, der cash flow nimmt entsprechend $z u$. Ist $\mathrm{S}$ kleiner als Null, verringern sich die Verbindlichkeiten des Wohnungsinvestors, gleichzeitig wird aber auch der entnahmefähige Betrag kleiner. 
Die Möglichkeit, Kredite aufnehmen zu können, ist für den Wohnungsinvestor nicht unbegrenzt. Bei Kapitalgesellschaften erfolgt die Beschränkung unmittelbar durch aktienrechtliche Bestimmungen, bei Personengesellschaften aus dem zumindest langfristig beobachtbaren Verhalten der Kreditgeber: die Geschäfts̄banken werden sicherlich nicht bereit sein, mehr als den Marktwert des in den Betreib investierten Vermögens zu beleihen. Eine hinreichende Bedingung dafür, daß diese Grenze nicht überschritten wird, ist, daB in jeder Periode nicht mehr als der Marktwert der Nettoinvestition $\mathrm{H}$ zuzüglich der periodenbezogenen Wertsteigerungen (abzüglich der periodenbezogenen Wertminderung) des Wohnflächenbestandes beliehen werden.

Obgleich für die Analyse der Laisser-Faire-Ǒkonomie irrelevant, empfiehlt es sich aus Gründen der Darstellung, der Nettoneuverschuldung schon an dieser stelle Schranken zu setzen. Wenn wir bedenken, daß der Marktwert der Nettoinvestition dem Barwert der mit $\dot{H}$ Wohnflächeneinheiten zusätzlich erzielbaren Mieterträge (abzüglich der abnutzungsbedingten Wertminderungen) entspricht und dieser Barwert aufgrund einer permanent. wachsenden Nachfrage nach Wohnleistungen (möglicherweise) von Periode zu Periode wächst, so muß im Finanzierungsplan des wohnungsinvestors die Restriktion

$$
S \leq \mathrm{P}_{\mathrm{H}} \dot{\mathrm{H}}+\dot{\mathrm{P}}_{\mathrm{H}} \mathrm{H}=\mathrm{P}_{\mathrm{H}}\left[\mathrm{F}^{\mathrm{d}} \varphi(\varepsilon)-(\alpha \delta \mathrm{H}-\mathrm{E})\right]+\dot{\mathrm{P}}_{\mathrm{H}} \mathrm{H}
$$

beachtet werden; hierbei steht $\mathrm{P}_{\mathrm{H}}$ für den Barwert der mit einer Wohnflächeneinheit netto erzielbaren Mieteinnahmen und $\dot{\mathrm{P}}_{\mathrm{H}}$ für dessen periodenbezogene, nachfragebedingte Änderung. Eine untere Schranke existiert für die Nettoneuverschuldung dagegen nicht: niemand verbietet es einem Unternehmer, seine Schulden zu tilgen oder Kapitalmarktpapiere zu erwerben. 
Aufbauend auf diesen Vereinbarungen läßt sich der in einer Periode für Entnahmezwecke zur Verfügung stehende Betrag A wie folgt beschreiben:

$$
A=m H-E-F^{d} \varepsilon-P_{B} F^{d}-r D+S .
$$

In (II.15) wurde der Preis des Investitionsgutes als "laufendes" Numéraire gewählt: $P_{I}$ ist $z u$ jedem beliebigen zeitpunkt $t$ des Planungshorizontes $\left(t_{0}, \infty\right)$ gleich Eins.

Dem FISHERschen Trennungstheorem folgend, setzen wir dem Wohnungsunternehmer die Maximierung des Marktwertes seiner Unternehmung zum $\mathrm{ziel}$. Der Marktwert ist derjenige Wert, den ein potentieller Käufer - in unserer Modellwelt wäre dies der repräsentative Haushalt - für dieses Unternehmen maximal zu zahlen bereit wäre; diese maximale Zahlungsbereitschaft wird ihrerseits durch die Vorteile - sprich: Entnahmen - geprägt, die das Unternehmen dem Käufer-Haushalt zu bieten vermag. Der Marktwert des Wohnungsunternehmens entspricht demnach der summe der Barwerte allen seiner heutigen und zukünftigen Entnahmen.

Der Wohnungsunternehmer wird nun versuchen, durch eine optimale wahl der zeitpfade für die Nettoneuverschuldung $S$, den Erhaltungsaufwand $E$, die Kapitalintensität $\varepsilon$ und den Bodenverbrauch $F^{d}$ der ihm gestellten Aufgabe der Marktwertmaximierung gerecht $z u$ werden. Rechentechnisch präsentiert sich dieser Optimierungskalkül als ein Problem der optimalen Kontrolle: $S, E, F^{d}$ und $\varepsilon$ sind die Kontrollvariablen, der Wohnflächenbestand $\mathrm{H}$ und der Fremdkapitalbestand $\mathrm{D}$ die dazugehörenden $\mathrm{Zu}-$ standsvariablen. Die $\mathrm{zu} H$ und $D$ korrespondierenden Bewegungsgleichungen lauten (unter Vernachlässsigung der Zeitindizes):

$$
\begin{array}{ll}
\text { (II.10) } & \dot{\mathrm{H}}=\mathrm{F}^{\mathrm{d}} \varphi(\varepsilon)-\alpha \delta \mathrm{H}+\mathrm{E}, \\
\text { (II.16) } & \dot{\mathrm{D}}=\mathrm{S} .
\end{array}
$$


Bevor wir uns dem Optimierungskalkül des Wohnungsinvestors zuwenden können, gilt es noch ein Problem rein formalen Charakters zu lösen: eine Existenzbedingung für die Lösung des oben geschilderten Kontrollproblems verlangt die stetigkeit der $\mathrm{Zu}-$ standsvariablen - sprunghafte Veränderungen des Wohnflächenbestandes $\mathrm{H}$ oder auch des Baulandbestandes B wären somit ausgeschlossen. Damit wäre aber die Existenz unbebauten Baulandes - sogenannter "Baulücken" - nach Beginn des Planungsproblems in $t_{0}$ schon allein aus mathematischen Gründen zwingend. $\mathrm{Da}$ es unter anderem aber ein ziel dieser Arbeit ist, zu untersuchen, ob das Horten von - das "Spekulieren" mit - Bauland nicht auch für das volkswirtschaftliche Ganze (und nicht nur für einige wenige Bodenspekulanten) vorteilhaft sein kann, müssen wir dem Wohnungsinvestor im Rahmen unserer Analyse die Möglichkeit einräumen, im Grenzfall den gesamten zum Zeitpunkt $t_{\circ}$ zur Bebauung freigegebenen Boden in $t_{0}$ auch tatsächlich bebauen $z u$ können.

Ein ähnliches Problem stellt sich im Zusammenhang mit dem Erhaltungsaufwand. Eine stetige Entwicklung des Wohnflächenbestandes würde bei gegebenem Zeitpfad der Neubauinvestitionen der Planung des Erhaltungsaufwandes von vornherein Schranken setzen. Will man das Geschehen am Wohnungs(bestands)markt realistisch beschreiben, so mu man auch aus diesem Grunde die Möglichkeit einer unstetigen Entwicklung des Wohnflächenbestandes in seinen Uberlegungen berücksichtigen.

Lösen lassen sich diese Probleme mit Hilfe eines formalen Tricks ${ }^{17)}$. Dieser besteht darin, daß wir zusätzlich zur tatsächlichen Zeit eine künstliche Zeit einführen. Während diese Kunstzeit abläuft, steht die tatsächliche Zeit still. Auf diese Weise gelingt es uns, eine realen Zeitpunkt "auseinanderzuziehen" und eine in der realen zeit stattfindende sprunghafte Veränderung der betrachteten Zustandsvariablen als stetigen Prozess zu beschreiben; Abb. 4 veranschaulicht diesen Sachverhalt graphisch.

17) KAMIEN/SCHWARTZ(1981), S.226 ff., SINN(1984). 
$\mathrm{Abb} .4$

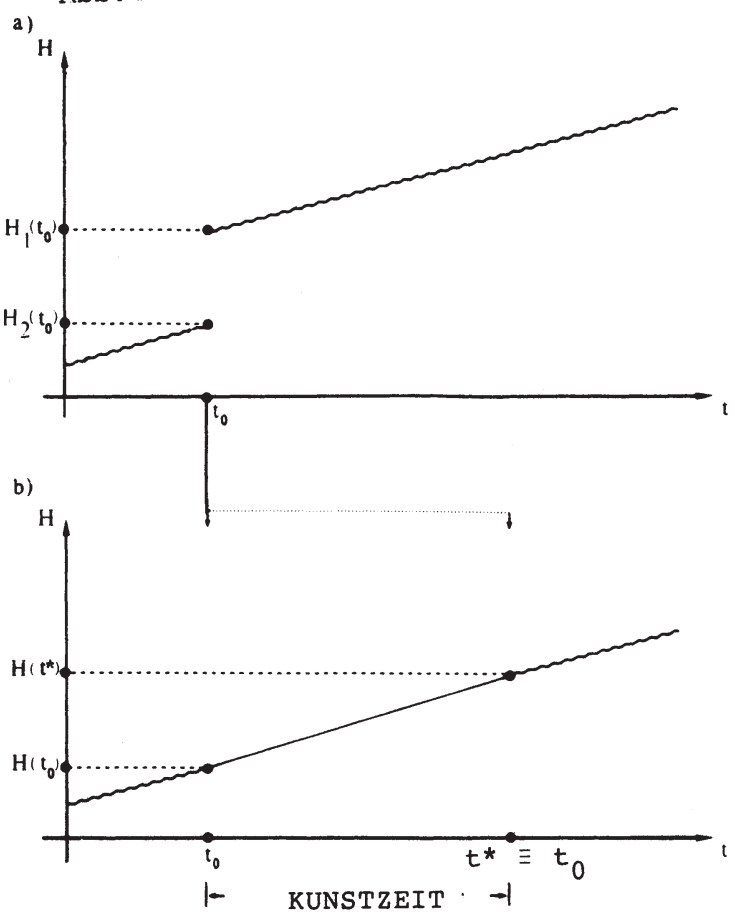

$t_{0}$ ist der Startpunkt unseres Planungsproblems. Wenn sich der Wohnungsinvestor $z \mathrm{u}$ diesem Zeitpunkt dazu entschließen sollte, den gesamten freien Bauboden zu bebauen, so wird der Wohnflächenbestand - wie in Skizze a) dargestellt - in $t_{0}$ sprunghaft von $\mathrm{H}_{1}$ auf $\mathrm{H}_{2}$ anwachsen. Um diesen sprung in einen stetigen Prozess zu transformieren, starten wir in $t_{0}$ die kunstzeitphase - die tatsächliche zeit bleibt in $t_{0}$ stehen -, innerhalb der sich nun die Entwicklung von $\mathrm{H}_{1} \mathrm{zu} \mathrm{H}_{2}$ kontinuierlich vollzieht (Skizze b)). Dieser fiktive Anpassungsprozess ist nach $\left(t *-t_{0}\right)$ künstlichen "Perioden" abgeschlossen; danach läuft die tatsächliche Zeit weiter. 
Der Tatsache, daß während der Kunstzeitphase $\left(t_{0}, t^{*}\right)$ weder Mieterträge realisiert werden noch abnutzungsbedingte Wertminderungen, Fremdkapitalzinsen oder Ausgaben für werterhaltende Maßnahmen anfallen, wohl aber Aufwendungen für Bauland und Bauleistungen $\mathrm{zu}$ beobachten sind und eventuell auch eine Nettoneuverschuldung stattfindet, wollen wir durch die Einführung eines Indikators $z$ Rechnung tragen, für den wir die folgende Konvention vereinbaren:

$$
\text { (II.17a) } \quad z=\left\{\begin{array}{l}
0 \text { für } t_{0} \leq t \leq t^{*} \\
1 \text { für } t \geq t^{*}
\end{array} .\right.
$$

Desselben Kunstgriffs wollen wir uns zur Beschreibung eines Sprungs in der Zustandsvariablen $\mathrm{H}$ bedienen, der aufgrund einer entsprechenden Planung werterhaltender Arbeiten zustandekomm: sollte sich der Wohnungsinvestor $\mathrm{zu}$ einem beliebigen Zeitpunkt $t_{1}$ entschließen, einen bestimmten Anteil der Differenz $H\left(t_{1}\right)-H\left(t_{1}\right)$ durch werterhaltende Arbeiten aufuholen, so stoppen wir die tatsächliche zeit in $t_{1}$ und starten eine Kunstzeit, in deren Verlauf die Bestandsanpassung erfolgt. Nach Abschluß dieses Anpassungsprozesses in $t * *$ läuft die tatsächliche Zeit weiter. Während der Kunstzeitphase $\left(t_{1}, t^{* *}\right)$ werden weder Mieteinnahmen erzielt, noch fallen abnutzungsbedingte Wertminderungen, Ausgaben für den Kapitaldienst oder Neubauinvestitionen an. Eine Nettoneuverschuldung ist dagegen möglich. Zur formalen Darstellung dieses Sachverhalts verwenden wir den Indikator $y$, für den annahmegemäß gi.lt:

(II.17b) $\quad y=\left\{\begin{array}{l}0 \text { für } t_{1} \leq t<t * * \\ 1 \text { sonst }\end{array}\right.$

Unter Beachtung von (II.17a) und (II.17b) lassen sich die periodenbezogenen Entnahmen dann auch wie folgt formulieren:

$$
A=y z(m H-r D)-y\left(F^{d} \varepsilon+P_{B} F^{d}\right)-z E+S
$$


Mit Hilfe von (II.18) ist es nun auch möglich, das Entscheidungsproblem des Wohnungsinvestors vollständig zu beschreiben:

(II.19)

$\left\{\mathcal{S}, \mathrm{Max}, \varepsilon, \mathrm{F}^{\mathrm{d}}\right\}$

$$
\begin{aligned}
M_{W} \equiv & \int_{t_{0}}^{t_{1}} A(t) \exp \left[-y z r\left(t-t^{*}\right)\right] d t \\
& +\int_{t_{1}}^{\infty} A(t) \exp \left[-y z r\left(t-t^{* *}\right)\right] \exp \left[-y z r\left(t_{1}-t^{*}\right)\right] d t
\end{aligned}
$$

unter den Bedingungen

(II. 2)

$$
H\left(t_{0}\right)>0
$$

(II. 3)

$$
D\left(t_{0}\right)>0 \text {, }
$$

(II.9)

$E\left\{\begin{array}{l}\geq 0 \text { für } H \leq H^{\max } \\ \leq \alpha \varepsilon_{H} \text { für } H=H^{\max }\end{array}\right.$

(II. 10)

$$
\dot{\mathrm{H}}=\mathrm{yF}^{\mathrm{d}} \varphi(\varepsilon)-\mathrm{z}(\mathrm{y} \alpha \delta \mathrm{H}-\mathrm{E}),
$$

(II. 14)

$$
S \leq P_{H}\left[y F^{d} \varphi(\varepsilon)-z(y \alpha \delta H-E)\right]+\dot{P}_{H} H,
$$

(II. 16)

$$
\dot{\mathrm{D}}=\mathrm{S},
$$

(II.17a)

$$
z=\left\{\begin{array}{l}
0 \text { für } t_{0} \leq t<t^{*} \\
1 \text { für } \quad t>t^{*}
\end{array},\right.
$$

(II.17b)

$$
y=\left\{\begin{array}{ll}
0 & \text { für } t_{1} \leq t<t * * \\
1 & \text { für } t \geq t * *
\end{array},\right.
$$

$$
A=y z(m H-r D)-y\left(F^{d} E+P_{B} F^{d}\right)-z E+S .
$$

Bei (II.19) handelt es sich um das formale Pendant der zuvor intuitiv abgeleiteten Idendität des Marktwertes $\mathrm{M}_{W}$ des Wohnungsunternehmens und des Barwertes der Entnahmen, die dieses Unternehmen seinen Eignern zukommen läßt. Da eine Abdiskon- 
tierung nur im Laufe der tatsächlichen Zeit vorgenommen werden darf, tauchen die Indikatoren $z$ und $y$ auch im Exponent des Diskontierungsterms auf. Als Diskontierungsfaktor selbst wurde der Kapitalmarktzins $r$ gewählt, den der Investor als Datum in sein Kalkül aufnimmt und der die Rendite der alternativen Finanzinvestition widerspiegelt. Hier zeigt sich die Funktion des Marktwertes (Ertragswertes) als relativer BewertungsmaBstab. Aus Gründen der rechentechnischen Vereinfachung wird in (II.19) die Konstanz des Realzinses im Zeitablauf unterstellt. Methodisch entspricht dies dem Fall einer kleinen offenen orkonomie, deren wirtschaftliche Entscheidungsträger das binnenwirtschaftliche Zinsniveau durch eigenes Handeln nicht beeinflussen können.

Die zu dem Kontrollproblem (II.19) gehörende Hamiltonfunktion lautet, ausgedrückt in laufenden Werten:

$$
\begin{aligned}
H= & y z(m H-r D)-y\left(F^{d} \varepsilon+P_{B} F^{d}\right)-z E+s \\
& +P_{H}\left[y F^{d} \varphi(\varepsilon)-z(y \alpha \delta H-E)\right] \\
& +\lambda_{D} S .
\end{aligned}
$$

$\mathrm{P}_{\mathrm{H}}$ ist der Schattenpreis des Wohnflächenbestandes; wir wollen $P_{H}$ im folgenden als Häuserpreis bezeichnen; entsprechend steht $\lambda_{D}$ für den Schattenpreis des unternehmerischen Fremdkapitalbestandes.

Die Werte der Kontrollvariablen $\mathrm{S}, \mathrm{E}, \varepsilon$ und $\mathrm{F}^{\mathrm{d}}$ müssen nun so gewählt werden, daß der Wert der Hamiltonfunktion zu jedem Zeitpunkt maximal wird. 
1.1. Der optimale Finanzierungsplan

Der Wohnungsinvestor kommt seinem ziel der Marktwertmaximierung solange nicht näher, solange er sich nicht darüber im Klaren ist, auf welche Weise er seine Investitionen finanzieren will. Die Frage nach der Finanzierungsform ist deshalb von so großer Bedeutung, weil die Maximierung des unternehmensmarktwertes die wahl des billigsten Finanzierungsweges zur unbedingten voraussetzung hat ${ }^{18}$ )

Dem Wohnungsinvestor bieten sich grundsätzlich immer die Alternativen, die zur Finanzierung seiner Immobilie erforderlichen Geldbeträge entweder aus eigenen Mitteln aufzubringen (für die Selbstfinanzierung wird im folgenden das Kürzel SF gewählt) oder auf dem Kreditwege zu beschaffen (FF steht im folgenden für diesen Finanzierungsweg) - denkbar ist auch eine beliebige Kombination aus Selbst- und Fremdfinanzierung. Die Selbstfinanzierung umfaßt hierbei sowohl die Möglichkeiten der Gewinnthesaurierung als auch die der Beteiligungs- bzw. Einlagenfinanzierung; im Falle der Beteiligungs- bzw. Einlagenfinanzierung ist der Entnahmebetrag in Gleichung (II.18) negativ.

Der optimale Umfang der Nettoneuverschuldung ist nun genau dann erreicht, wenn der Wert der Hamiltonfunktion durch eine Variation der Kontrollvariable $s$ nicht mehr erhöht werden kann. Wegen der Verschuldungsschranke aus (II.14) und der Linearität der Hamiltonfunktion bezüglich $\mathrm{S}$ kommen als optimale Lösung

18) Diesem engen Zusammenhang zwischen Finanzplanung und Investitionsplanung wird in der steuertheoretischen Literatur oft nur sehr unzureichend Rechnung getragen. Meistens wird eine bestimmte Finanzierungsform unterstellt, ohne zu überlegen, ob diese Art der Finanzierung auch wirklich die optimale ist. Daß sie es häufig nicht ist, ist die Ursache für viele Fehlurteile über die Wirkung von Steuern (vgl. beispielsweise die Diskussion um die Inzidenz der Kapitaleinkammensbesteuerung bei HARBERGER(1963), MCLURE (1971), BALCENTINE (1978) und SINN(1932). 
nur die folgenden Möglichkeiten in Frage ${ }^{19}$ ?

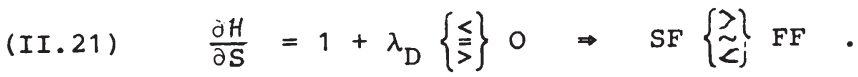

Um zu wissen, welcher der in (II.21) beschriebenen Finanzierungspläne der optimale ist, muB man den Wert des Schattenpreises $\lambda_{D}$ kennen; für diesen gilt

$$
\begin{aligned}
\lambda_{D} \equiv \frac{\partial M_{W}}{\partial D}= & \int_{t_{0}}^{t_{1}}-y z r \cdot \exp [-y z r(t-t *)] d t \\
& +\int_{t_{1}}^{\infty}-y z r \cdot \exp [-y z r(t-t * *)] \exp \left[-y z r\left(t_{1}-t^{*}\right)\right] d t \\
= & -1 .
\end{aligned}
$$

Aufgrund dieses Ergebnisses sind in (II.21) die Fälle $\partial H / \partial S \gtrless O$ ausgeschlossen; dementsprechend ist die Optimalitätsbedingung (II.21) für beliebige Werte der Nettoneuverschuldung erfüllt, vorausgesetzt, diese bewegen sich innerhalb des Kontrollbereichs von $\mathrm{s}$.

Somit läßt sich als erstes Ergebnis unserer Analyse der Laisser-Faire-Welt folgendes festhalten:

Die optimale verschuldungspolitik des wohnungsinvestors ist nicht eindeutig determiniert - der wohnungsinvestor ist zwischen den sich ihm eröffnenden Finanzierungswegen indifferent

Die ökonomische Intuition, die sich hinter dieser Aussage verbirgt, läßt sich leicht vermitteln; hierzu muß man sich lediglich die Vor- und Nachteile vor Augen führen, die dem Eignerhaushalt erwachsen, wenn der wohnungsinvestor die Finanzierungsstruktur der Wohnungsinvestition zugunsten der Fremafinanzierung verändert; wird beispielsweise 1 D-Mark Eigenkapi-

19) Darüberhinaus muß auch die Bedingung $-\partial H / \mathrm{D} D=\dot{\lambda}_{D}-r \lambda_{D}$ erfüllt sein.

Da diese Bedingung aber keine zusätzlichen Informationen zu liefern vermag, wird sie im Text vernachlässigt. 
tal durch 1 D-Mark Fremakapital ersetzt, so erhöhen sich unmittelbar die Ausschüttungen an den Eignerhaushalt um 1 D-Mark. Diese kann zum herrschenden Marktzinssatz I am Kapitalmarkt angelegt werden und dem Eignerhaushalt zu zusätzlichen Erträgen aus Wertpapiervermögen verhelfen. Andererseits entstehen dem Wohnungsunternehmer im zuge dieser Änderung aber zusätzliche Zinskosten in der gleichen Höhe, die die dem Eignerhaushalt zugutekommenden Entnahmebeträge in vollem Umfang mindern. Per saldo bleibt: die Vermögensposition des Eignerhaushalts demnach unverändert. Da der Wohnungsinvestor aber nur dann einem bestimmten Finanzierungsweg den Vorzug geben wird, wenn sich dadurch für den sektor der privaten Haushalte Vermögensvorteile erzielen lassen - diese Aussage läßt sich unmittelbar aus dem Trennungstheorem ableiten - wird er in der steuerfreien Welt keinen der ihm offenstehenden Finanzierungswege eindeutig präferieren. 20

\subsection{Der optimale werterhaltungsplan}

Von der Festlegung des Zeitpfades für die werterhaltenden Maßnahmen hängt die Entwicklung des Althausbestandes im Zeitablauf ab. Da die Hamiltonfunktion in (II.2O) linear in E ist, mu $\beta$ auch hier wiederum mit Randlösungen für die Kontrollvariable gerechnet werden.

Bei der Bestimmung des optimalen Werterhaltungsprogramms muß grundsätzlich von der allgemeinen optimalitätsbedingung

$$
\frac{\partial H}{\partial E}=\frac{\partial H}{\partial E}+\frac{\partial H}{\partial S} \frac{\partial S}{\partial E}
$$

20) In der Literatur wird oft ein mit zunehmendem Verschuldungsgrad ansteigender Sollzins unterstellt, mit dessen Hilfe dann eine optimale Fremdfinanzierungsquote errechnet werden kann. Daß ein steigender Sollzins aber nicht unbedingt und immer auch einen steigenden Effektivzins impliziert und die Verschuldung keineswegs immer bei steigendem Sollzins an Attraktivatät verliert, zeigt HELLWIG(1981) mit der Verallgemeinerung des Modigliani-Miller-Theorems;- vgl. auch MODIGLIANI/MIILER(1958), SINN(1982), Kapitel II. 
ausgegangen werden. Denn mit dem Umfang werterhaltender Maßnahmen schwankt auch der Beleihungswert des bebauten Grundstücks, und zwar - wie aus (II.14) hervorgeht - nach Maßgabe des partiellen Differentials

$$
\text { (II.24) } \quad \frac{\partial S}{\partial E}=2 P_{H} E \text {. }
$$

Da der Wohnungsinvestor aus der Nutzung dieses zusätzlichen Beleihungsspielraums aber keinen Vorteil zu ziehen vermag es gilt $\partial H / \partial S=0$ - vereinfacht $\operatorname{sich}($ II.23) $\mathrm{zu}$

(II. 23)'

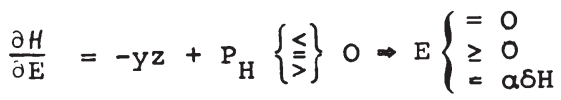

Nach Aussage von (II.23)' kompensiert der Wohnungsinvestor in jeder Periode die abnutzungsbedingten Wertminderungen nur dann in voller Höhe durch entsprechende Renovierungsarbeiten, wenn sichergestellt ist, daß die Kosten dieser Arbeiten durch die zusätzlichen Mieterträge gedeckt werden, die die Substanzerhaltung letztendlich ermöglicht. (II.23)' fordert diese Kostendeckung für die marginale Einheit Erhaltungsaufwand: für $t \geq t *$ gilt $z=1$, dies sind die Kosten einer Einheit Werterhaltung. Im Gegenzug wird durch diese werterhaltenden Arbeiten eine Wohnflächeneinheit in ihren Neuzustand versetzt, durch deren Vermietung sich zusätzliche Mieterträge in Höhe von $\mathrm{P}_{\mathrm{H}}$ D-Mark - gemessen in Gegenwartswerten - erzielen lassen: für $P_{H}$ - den Schattenpreis des Wohnflächenbestandes - gilt

$$
\begin{aligned}
P_{H}\left(t_{0}\right) \equiv \frac{\partial M_{W}\left(t_{0}\right)}{\partial H\left(t_{0}\right)}= & \int_{t_{0}}^{t_{1}} y z \frac{\partial H(t)}{\partial H\left(t_{0}\right)} m\left(t_{0}\right) \exp \left[-y z r\left(t-t^{*}\right)\right] d t \\
& +\int_{t_{1}}^{\infty} y z \frac{\partial H(t)}{\partial H\left(t_{0}\right)} m\left(t_{0}\right) \exp \left[-y z r\left(t-t^{* *}\right)\right] \cdot \\
\exp \left[-y z r\left(t-t^{*}\right)\right] d t . & 21)
\end{aligned}
$$

21) Wegen der Annahme der vollkommenen Konkurrenz gilt in (II.25) $\mathrm{d} m\left(t_{0}\right) / \mathrm{uH}\left(t_{0}\right)=0$. 
Wegen $\partial H(t) / \partial H\left(t_{0}\right)=\exp \left[-\alpha \delta\left(t-t_{0}\right)\right]-$ zum Zeitpunkt $t$ sind von der marginalen Wohnflächeneinheit $\partial H\left(t_{0}\right)$ infolge abnutzungsbedingter Substanzminderungen noch genau $\partial \mathrm{H}\left(t_{0}\right) \exp \left[-\alpha \delta\left(t-t_{0}\right)\right]$ Wohnflächeneinheiten übrig - errechnet man sich aus (II.25) leicht

$$
P_{H}\left(t_{O}\right)=\frac{m}{r+a \delta}
$$

(II.26) Entspricht der vertrauten Kapitalisierungsformel der Erträge eines Wertpapiers mit unendlicher Laufzeit: im Zähler des Quotienten stehen die Bruttoerträge der Vermögensform "Wohnimmobilie", der Nenner weist die opportunitätskosten dieser Vermögensform in Gestalt entgangener Kapitalmarkterträge und abnutzungsbedingter Wertverluste aus.

Ist der Häuserpreis kleiner als Eins, ist es für den Wohnungsinvestor nicht lohnenswert, werterhaltende Arbeiten auszuführen. Die Werterhaltung wird erst zu dem Zeitpunkt rentabel, zu dem der Häuserpreis den Wert Eins annimmt bzw. übertrifft. Genau zu diesem Zeitpurkt wird es zu einer sprunghaften Änderung des Wohnflächenbestandes kommen, da es sich für den Wohnungsinvestor nun lohnt, alle in der Vergangenheit unterlassenen Renovierungsarbeiten nachzuholen: die sprunghafte Anpassung des Wohnflächenbestandes $H\left(t_{1}\right)$ an den maximal möglichen Bestand $\max$

$\max _{H\left(t_{1}\right)}$ läßt sich innerhalb der Kunstzeitphase ( $\left.4, t^{* *}\right)$ als stetiger Prozeß darstellen. Falls $P_{H}$ jenseits von $t * \%$ größer als Eins ist, wird der Umfang werterhaltender Arbeiten einer beliebigen Periode durch (II.9) auf $\mathrm{E}=\alpha \delta \mathrm{H}$ beschränkt.

Da $\mathrm{P}_{\mathrm{H}}$, wie wir im folgenden noch sehen werden, im Zeitablauf stetig an wert gewinnt und den Betrag einer D-Mark nach absehbarer Zeit zwangsläufig übertreffen muß, wollen wir aus Gründen der Vereinfachung annehmen, daß zu jedem von uns $z u$ betrachtenden Zeitpunkt $P_{H}>1$, deshalb $y=1$ und somit im optimum 
(II.27) $E=\left\{\begin{array}{l}0 \text { für } t_{0} \leq t<t * \\ a \delta H \text { für } t \geq t *\end{array}\right.$

gilt:

In jeder Periode werden die abnutzungsbedingten Substanzminderungen des Gebäudekapitalstocks durch Erhaltungsarbeiten vollständig wiederaufgeholt.

1.3. Der optimale wohnungsneubauplan

Die Investitionsplanung spielt im Rahmen der Unternehmenspolitik mit die wichtigste Rolle. Die optimale Neubauentscheidung verlangt vom Wohnungsinvestor, daß er sowohl den Zeitpfad für den Bodenverbrauch $\left\{E^{d}\right\}_{t_{0}}^{\infty}$ als auch den für die Kapitalintensität der Bebauung $\{e\}_{t_{0}}^{\infty}$ so wählt, daß diese Wahl den Anforderungen des Planungsproblems aus (II.19) genügt.

Um den EinfluB der Finanzplanung auf die Neubauentscheidung des Wohnungsinvestors korrekt nachvollziehen $\mathrm{zu}$ können, ist es erforderlich, von den allgemeinen Optimalitätsbedingungen

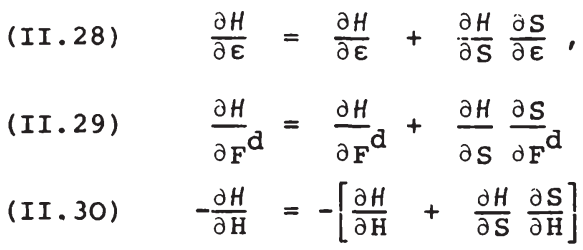
auszugehen. 
Hierbei ist zusätzlich $\mathrm{zu}$ beachten, daß infolge der in (.II.14) formulierten Finanzierungsschranke die partiellen Differentiale

$$
\begin{aligned}
& \frac{\partial S}{\partial \varepsilon}={ }_{H^{\prime}} F^{d} \varphi^{\prime}(\varepsilon), \\
& \text { (II.32) } \frac{\partial S}{\partial F^{d}}=P_{H^{\varphi}}(\varepsilon) \text {, }
\end{aligned}
$$$$
\text { (II.33) } \quad \frac{\partial S}{\partial H}=-P_{H} \alpha \delta+\dot{P}_{H}
$$

Gültigkeit besitzen: Neubauinvestitionen und (nachfragebedingte) Wertsteigerungen des Grundstïckvermögens steigern die Kreditwürdigkeit des Wohnungsunternehmers, (abnutzungsbedingte) Wertminderungen des Grundstücks schränken sie ein.

Im Falle der Laisser-Faire-ðkonomie vereinfachen sich die Optimalitätsbedingungen (II.28), (II.29) und (II.30) nun aber insofern, als in Abschnitt II.1.1. die Finanzierungsindifferenz des Wohnungsinvestors, d.h. $\partial H / \partial S=0$, festgestellt wurde.

Unter Beachtung dieser Zusammenhänge lauten die Bedingungen für die optimale Neubauentscheidung wie folgt:

Die gewählte Kapitalintensität der Bebauung ist dann die optimale, wenn

$$
\partial H / \partial \varepsilon \stackrel{!}{=} O=-F^{d}+F^{d} P_{H} \varphi^{\prime}(\varepsilon)
$$

erfullt ist 22). Dividiert man (II.34) durch $\mathrm{F}^{\mathrm{d}}$ und stellt anschließend um, so erhält man die Marginalbedingung

$$
P_{H} \varphi^{\prime}(\varepsilon)=1 \text {. }
$$

(II.35) läßt sich in einer vertrauten mikroökonomischen Weise interpretieren: auf der linken seite des Gleichheitszeichens steht das Wertgrenzprodukt des Produktionsfaktors "Bauleistun22) Aufgrund der strikten Quasikonkavität der Produktionsfunktion muß es eine innere Lösung für $\varepsilon$ geben. 
gen" -es gilt $\partial\left[\mathrm{P}_{H} F^{d} \varphi(\varepsilon)\right] / \partial I=P_{H} \varphi^{\prime}(\varepsilon)$. Auf der rechten seite des Gleichheitszeichens steht der Kaufpreis dieser marginalen Leistungseinheit. Offensichtlich muB im Investitionsoptimum das Wertgrenzprodukt des Faktors "Bauleistungen" übereinstimmen mit dessen Grenzkosten.

$\mathrm{zu}$ einer ähnlichen Bedingung gelangen wir, wenn wir den Zeitpfad für die optimale Nachfrage nach Bauboden bestimmen wollen. Der Grund, warum dieses Optimierungsproblem überhaupt akut wird, ist in der Tatsache $z u$ sehen, daß der Grundstlicksbestand langfristig konstant ist. Deshalb verringert Boden, der heute bebaut wird, das Baulandangebot zukünftiger Perioden und schränkt auf diese weise auch die zukünftigen Wohnungsneubaualternativen ein. Wenn heute "zuviel" Boden bebaut wird, ist ein vorgegebenes optimales Wohnflächenbestandsangebot in zukünftigen Perioden dann nur noch über eine suboptimale - weil zu kostspielige - Erhöhung der Kapitalintensität der Bebauung zu erreichen.

Infolge der Linearität der Hamiltonfunktion in $\mathrm{F}^{\mathrm{d}}$ lautet die Bedingung für aie optimale Baubodennachfrage des Wohnungsinvestors

$$
\partial H / \partial F^{d}=-\varepsilon-P_{B}+P_{H} \varphi(\varepsilon)\left\{\begin{array}{l}
< \\
=
\end{array}\right\} O \Rightarrow F^{d}\left\{\begin{array}{l}
= \\
\geq
\end{array}\right\} 0
$$

Un (II.36) sinnvoll interpretieren zu können, muß man wissen, daß sich hinter dem Ausdruck $P_{H} \varphi(\varepsilon)-\varepsilon$ das Wertgrenzprodukt des Bodens bei āer Schaffung neuen Wohnraums verbirgt 23) Das Wertgrenzprodukt des Bodens ist zugleich Ausdruck der maximalen Zahlungsbereitschaft des Wohnungsinvestors für die letzte zu bebauende Grundstücksflächeneinheit. Bedingung (II.36) sagt uns, daß es nur dann zu Grundstückstransaktionen kommen wird, wenn

23) Unter Beachtung von (II.35) gilt

$$
\begin{aligned}
\partial\left[\mathrm{P}_{\mathrm{H}} F^{d} \varphi(\varepsilon)\right] / \partial F^{d} & =P_{H} \varphi(\varepsilon)-P_{H} F^{d} \varphi^{\prime}(\varepsilon)\left[-I /\left(F^{d}\right)^{2}\right] \\
& =P_{H} \varphi(\varepsilon)-P_{H^{\prime}} \varphi^{\prime}(\varepsilon) \varepsilon \\
& =P_{H} \varphi(\varepsilon)-\varepsilon
\end{aligned}
$$


das Wertgrenzprodukt des Bodens wertmäßig übereinstimmt mit dem von den Bodenmaklern für die Flächeneinheit Bauland geforderten Preis $P_{B}$. Reicht die Zahlungsbereitschaft der Wohnungsin vestoren nicht an $P_{B}$ heran, findet kein Handel mit Bauland und folglich auch kein Wohnungsneubau statt.

In (II.36) wird die Möglichkeit $\partial H / \partial \mathrm{F}^{\mathrm{d}}>0$ ausgeschlossen; würde man diese Möglichkeit zulassen, wäre das Wertgrenzprodukt des Bodens größer als der geforderte Bodenpreis; entsprechend würde es sich für den Wohnungsinvestor lohnen, zusätzlichen Boden zu kaufen und ihn zu bebauen; der Wert der Hamiltonfunktion würde in diesem Falle aber steigen - und zwar solange, bis sich Bodenpreis und maximale Zahlungsbereitschaft aufgrund der hohen Nachfrage nach Bauland einander angeglichen hätten. Demnach ist der Fall $\partial H / \partial F^{d}>0$ mit der Existenzbedingung für ein Maximum der Hamiltonfunktion bezüglich des Bodenverbrauchs nicht zu vereinbaren.

Bedingung (II.36) läßt sich vereinfachen; unter Berücksichtigung der Tatsache, daB die Produktionsfunktion in (II.5) durch konstante partielle Produktionelastizitäten gekennzeichnet ist, erhält man für die partielle Produktionselastizität a des Faktors "Bauleistungen" die Beziehung

$$
\alpha=\frac{\varphi^{\prime}(\varepsilon)}{\varphi(\varepsilon)} \varepsilon=\text { konstant }>0 \text {. }
$$

Wegen der Annahme der linearen Homogenität und streng positiver Faktorgrenzprodukte gilt darüberhinaus

$$
0<\alpha=1-\beta<1,
$$

wobei B für die partielle Produktionselastizität des Faktors "Boden" steht.

24) Diese Beziehung kann man sich anhand einer Produktionsfunktion des cobb-Douglas-Typs leicht ableiten. 
Unter Berücksichtigung von (II.37) und (II.38) läBt sich (II.36) umformen $\mathrm{zu}$

$$
\begin{aligned}
& \partial H / \partial F^{d}=\frac{\varphi(\varepsilon)}{\varphi^{\prime}(\varepsilon)}-\varepsilon-P_{B} \\
& =\frac{1}{\alpha} \varepsilon-\varepsilon-\mathrm{P}_{\mathrm{B}} \\
& =\frac{\beta}{\alpha} \varepsilon-P_{B}\left\{\begin{array} { l } 
{ < \} } \\
{ = j }
\end{array} 0 \Rightarrow F ^ { d } \left\{\begin{array}{l}
= \\
\geq j
\end{array} 0 .\right.\right.
\end{aligned}
$$

Diese Umformung verhilft uns zu einer für unseren weiteren Analyseweg sehr wichtigen Erkenntnis: wie groß die gewählte Kapitalintensität der Bebauung ist, hängt ab von der Relation "Bodenpreis : Bauleistungspreis"- der Bauleistungspreis $P_{I}$ wurde in unserer Analyse als Numéraire gewählt - und vom Verhältnis der beiden partiellen Produktionselastizitäten. Dagegen wird die zeitliche Entwicklung von $\varepsilon$ infolge der Konstanz von $\alpha$ und $\beta$ alleine von der zeitlichen Entwicklung der Preisrelation $P_{B}$ bestimmt.

Die dritte notwendige Bedingung für das Wohnungsneubauoptimum verlangt für die partielle Ableitung der Hamiltonfunktion nach der Zustandsvariablen $\mathrm{H}$ die Beziehung

$$
\text { (II.40) } \quad-\frac{\partial H}{\partial H}=\dot{\mathrm{P}}_{\mathrm{H}}-\operatorname{zrP}_{\mathrm{H}}=-\mathrm{z}\left(\mathrm{m}-\alpha \delta \mathrm{P}_{\mathrm{H}}\right) .
$$

Durch einfaches Umstellen und unter Beachtung der Bedingungen für den Kunstzeitindikator $z$ erhält man hieraus die das optimum kennzeichnenden Wachstumsraten des Häuserpreises:

$$
\begin{array}{lll}
\text { (II. 42a) } & \hat{\mathrm{P}}_{\mathrm{H}}=0 & \text { für } t \leq t<t *, \\
\text { (II. 42b) } & \hat{\mathrm{P}}_{\mathrm{H}}=-\frac{m}{\mathrm{P}_{\mathrm{H}}}+r+\alpha \delta & \text { für } \quad t \geq t^{*} .
\end{array}
$$

Gleichung (II.42b) ist die bekannte Bedingung für ein intersektorales Arbitragegleichgewicht: das Volumen des im Wohnungssektor gebundenen Vermögens ist genau dann optimal, wenn die letzte in ein bebautes Grundstück investierte D-Mark denselben 
Nettoertrag erwirtschaftet, den man durch eine alternative Anlage dieser D-Mark am Kapitalmarkt hätte erzielen können. Die Rendite der Finanzinvestition betrüge hierbei $r$ D-Mark, während sich der Nettoertrag der Wohnungsinvestition zusammensetzt aus der Bruttorendite (= Bruttomiete bezogen auf den Häuserpreis) der Wohnungsinvestition abzüglichen der anteiligen Abschreibungsverluste ab und zuzüglich der wertsteigerung der mit der marginalen D-Mark finanzierten Wohnflächeneinheit (löst man (II.42b) nach $m / P_{H}$ auf, so erhält man die aus der Kapitaltheorie vertraute user cost of capital-Formel). Diese Bedingung muß zu jedem Zeitpunkt des Planungshorizonts $(t *, \infty)$ erfüllt sein.

Im Laufe der Kunstzeitphase $\left(t_{0}, t^{*}\right)$ ändert sich der Häuserpreis dagegen nicht - dies geht aus Bedingung (II.42a) hervor. Wäre diese Bedingung nicht erfüllt und würde beispielsweise $\hat{\mathrm{P}}_{\mathrm{H}}>0$ gelten, würde es sich für den Unternehmenseigner in $t_{0}$ lohnen, sein Portefeuille zugunsten des Wohnimmobilienvermögens umzuschichten, da während der Kunstzeitphase $\left(t_{0}, t^{*}\right)$ annahmegemäß keine Erträge aus Wertpapiervermögen erzielt werden. Entsprechend könnte es sich bei dem durch die Bedingungen (II.41a) und (II.41b) beschriebenen Zustand nicht um ein Portefeuillegleichgewicht handeln.

Schließlich muß im Planungsproblem des Bauherrn auch noch die Transversalitätsbedingung

$$
\text { (II.43) } \quad \lim _{t \rightarrow \infty}\left[P_{H}(t) H(t) \exp \left(-r\left(t-t_{0}\right)\right)\right]=0
$$

erfüllt sein. Sie ist eine notwendige Voraussetzung dafür, daß der Marktwert des Wohnungsunternehmens einen endlichen Wert annimmt und insoweit eine Lösung des betrachteten Planungsproblems überhaupt existiert.

\section{Das Planungsproblem des Bodenmaklers}

Der Optimierungskalkül des Wohnungsinvestors wurde unter der Annahme beschrieben, daß die Entscheidung über das Bodenangebot bereits gefallen ist. Offensichtlich hängt die optimale Investitionsentscheidung des Bauherrn und damit die Entwicklung des 
Wohnflächenbestandes entscheidend davon $a b$, wieviel Bauland der Bodenmakler $\mathrm{zu}$ welchem Zeitpunkt und $\mathrm{zu}$ welchem Preis anzubieten bereit ist. Der Frage, welche Faktoren diese Angebotsbereitschaft bestimmen, wollen wir uns im folgenden zuwenden.

AnnahmegemäB untersteht der Baulandbestand $B\left(t_{0}\right)$ von $A n-$ fang des Planungsproblems an der Verfügungsmacht des repräsentativen Bodenmaklers, der seinerseits im Auftrag des repräsentativen privaten Haushalts - des Bodeneigentümers - tätig ist.

Da die zur Bebauung freigegebenen Grundstücke keinem anderen Zweck als dem der Bebauung zugeführt werden dürfen, besteht die einzige Einnahmequelle des Bodenmaklers im Verkauf dieser Grundstücke. Die Verkaufserlöse selbst fließen an den privaten Haushalt und können von diesem für Konsumzwecke oder für Zwecke der Vermögensbildung verwendet werden.

Das erste $\mathrm{Ziel}$ des Bodenmaklers muß es deshalb sein - sofern er die ihm übertragenen Dispositionsgewalt nicht verlieren will - den Zeitpfad des Bodenangebots $\left\{F^{a}\right\}_{t_{0}}^{\infty}$ in einer Weise innerhalb des Planungshorizonts $\left(t_{0}, \infty\right) \mathrm{zu}$ plazieren, daB der Barwert der Verkaufserlöse möglichst groß wird.

Die zieladäquate Handlungsweise des Bodenmaklers läßt sich formal wiederum als das Ergebnis eines Kontrollproblems beschreiben. Da es sich auch bei dem repräsentativen Bodenmakler annahmegemäß um einen vollständigen Konkurrenten handelt, der die Zeitpfade des Marktzinses und des Bodenpreises als von ihm nicht beeinflußbare Daten hinnimmt, berechnet sich der Barwert $M_{B}$ der Verkaufserlöse ${ }_{B} F^{a}$ wegen $y=1$ gemäß der Formel

$$
M_{B}\left(t_{0}\right)=\int_{t_{0}}^{\infty} P_{B}(t) F^{a}(t) \exp \left[-z r\left(t-t^{*}\right)\right] d t .
$$

Hierbei gilt für $\mathrm{z}$ die schon weiter oben getroffene Vereinbarung

(II.17a) $\quad z=\left\{\begin{array}{ll}0 & \text { für } t_{0} \leq t<t^{*} \\ 1 & \text { für } t \geq t^{*}\end{array}\right.$.

Die Zustandsvariable des Kontrollproblems ist offensichtlich der Bestand B an baureifen Grundstücken. Da wir unterstellt haben, daB eine einmal durchgeführte Bebauung nicht mehr rückgängig gemacht werden kann, vermindert sich dieser 
Bestand in jeder Periode im Ausmaß der Bodenverkäufe. Es gilt deshalb die Bewegungsgleichung

$$
\dot{\mathrm{B}}=-\mathrm{F}^{\mathrm{a}}, \mathrm{F}^{\mathrm{a}} \geq 0
$$

Mit $F^{a}$ als Kontrollvariable lautet das vollständig formulierte Planungsproblem des Bodenmaklers

(II. 46)

$$
\left\{F^{a}\right\} \quad M_{B}\left(t_{0}\right)
$$

unter den Bedingungen

$$
\text { (II. 1) }
$$

$$
\mathrm{B}\left(\mathrm{t}_{0}\right)>0 \text {, }
$$

$$
z=\left\{\begin{array}{l}
0 \text { für } t_{0} \leq t<t * \\
1 \text { für } \quad t \geq t *
\end{array},\right.
$$

$$
\dot{\mathrm{B}}=-\mathrm{F}^{\mathrm{a}} \text {. }
$$

Unter Verwendung der Informationen aus (II.44) läßt sich die in laufenden Werten definierte Hamiltonfunktion für dieses Planungsproblem formulieren:

$$
H=P_{B} F^{a}-\lambda_{B} F^{a} ;
$$

$\lambda_{B}$ steht hierbei für den Schattenpreis des Baulandbestandes. Wie im ersten Abschnitt dieses Kapitals lassen sich die für ein Maximum notwendigen Bedingungen über die partielle Differentiation der Hamiltonfunktion nach den Kontroll- und Zustandsvariablen ermitteln. Man errechnet auf diesem Wege für eine innere Lösung die Bedingungen

$$
\begin{array}{ll}
\text { (II.48) } & \frac{\partial H}{\partial \underline{T}^{\mathrm{a}}}=\mathrm{P}_{\mathrm{B}}-\lambda_{\mathrm{B}} \stackrel{!}{=} 0 \\
\text { (II.49) } & \frac{\partial H}{\partial \mathrm{B}}=\dot{\lambda}_{\mathrm{B}}-\operatorname{zr} \lambda_{\mathrm{B}}=0 .
\end{array}
$$


Nach (II.48) stimmt im Optimum der Schattenpreis des Baubodens mit dessen Verkaufspreis überein; wenn man diese Beziehung in (II.49) substituiert, erhält man als notwendige Bedingungen für das optimale Baulandangebot

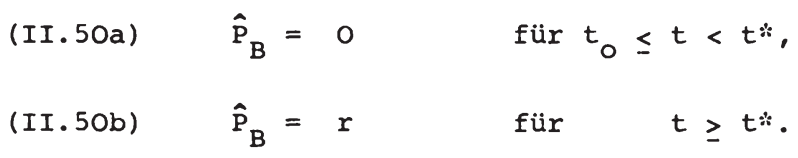

(II.50a) ist das Pendant zu Bedingung (II.42a) aus dem Planungsproblem des Wohnungsinvestors. Sie verlangt die Konstanz des Bodenpreises während der Kunstzeitphase.

Für uns von größerem Interesse ist Bedingung (II.5Ob). Sie verlangt, daB im Bodenmarktgleichgewicht - wenn ein Handel mit Bauland stattfinden soll - der Bodenpreis im Zeitablauf mit einer Rate zunehmen muß, deren Höhe dem Wert des Kapitalmarktzinses entspricht; (II.50) ist die aus der Theorie erschöpfbarer Ressourcen wohlbekannte HOTELLING-Regel ${ }^{25}$ ).

Folgende ökonomische Zusammenhänge verbergen sich hinter dieser Regel: Wie wir aus dem Planungsproblem des Kapitels I.I.1. wissen, kann es für den Wohnungsunternehmer unter Umstärden von Vorteil sein, mit dem Erwerb der zur Bebauung freigegebenen Bodenareale $\mathrm{zu}$ warten und erst $\mathrm{zu}$ einem späteren zeitpunkt ein auf die dann gegebenen Nachfrageverhältnisse zugeschnittenes Gebäude zu errichten. Offensichtlich setzt die Realisierung dieser Pläne die Bereitschaft des Bodenmaklers voraus, das zur Bebauung vorgesehene Areal bis zu diesem späteren Zeitpunkt in seinem Bestand an baureifen Grundstücken zu halten. Daß sich der Wohnungsinvestor diese Bereitschaft durch die Zahlung eines im Zeitablauf steigenden Bodenpreises erkaufen muB, uberrascht nicht, entstehen dem Bodenmakler durch die Hortung von Bauland doch Opportunitätskosten in Form entgangener Kapitalmarkterträge: hätte er seinen gesamten Bestand an baureifen Grundstücken zum Zeitpunkt $t_{0}$

25) HOTELTING (1931). 
verkauft und den dabei realisierten Verkaufserlös in Finanzaktiva investiert, so hätte er Periode um Periode einen Vermögenszuwachs nach Maßgabe des Marktzinssatzes $r$ erzielen können. Für diese Opportunitätskosten muß ihn der Wohnungsinvestor entschädigen - und die HOTELLING-Regel sagt uns, wie hoch diese Entschädigung ausfallen muß: die Zahlungsbereitschaft des Wohnungsinvestors für Bauland mu von Periode zu Periode um jeweil $r$ Prozent zunehmen, wenn er zu jedem Zeitpunkt jenseits von $t_{0}$ Wohnungsneubauinvestitionen durchführen möchte. Wächst diese Zahlungsbereitschaft nämlich mit einer geringeren Rate etwa weil mit einer auf Dauer relativ schwach ansteigenden Nachfrage nach Wohnleistungen gerechnet wird - ist es für den Bodenmakler rational, den gesamten vorhandenen Bauboden sofort zu verkaufen und den Verkaufserlös zum herrschenden $\mathrm{z}$ inssatz am Kapitalmarkt anzulegen; die dadurch erzielbare Rendite wäre allemal höher als jene, die er durch eine Hortung von Bauland würde erzielen können. Liegt der prozentuale Zuwachs an Zahlungsbereitschaft dagegen über dem Kapitalmarktzins etwa weil in zukunft mit einer stark zunehmenden Nachfrage nach Wohnleistungen gerechnet wird - erweist sich das Horten von Bauland als die rentabelste Strategie; es lohnt sich nun für den Grundstücksmakler, den Verkaufszeitpunkt solange hinauszuschieben, bis $\hat{\mathrm{P}}_{\mathrm{B}}=\mathrm{r}$ gilt.

Zu einem Verkauf von Bauboden und damit zu einer Neubautätigkeit im Laufe der realen zeit $\left(t^{*}, \infty\right)$ wird es folglich nur zu solchen Zeitpunkten kommen, zu denen der Marktpreis des Bodens mit einer Rate in Höhe von $r$ wächst.

Als dritte notwendige Bedingung für die Existenz einer Lösung des Planungsproblems unseres Bodenmaklers muB schlieBlich noch die Transversalitätsbedingung

(II.51) $\quad \lim _{t \rightarrow \infty}\left[\mathrm{P}_{B}(t) B(t) \exp \left[-\operatorname{zr}\left(t-t_{0}\right)\right]\right]=0$ erfüllt sein. 
3. Bedingungen des Marktgleichgewichts - Charakteristika einer paretooptimalen Allokation

Aufbauend auf den Analyseergebnissen für die Planungsprobleme des Wohnungsinvestors und des Bodenmaklers lassen sich nun auch die Bedingungen für die Gleichgewichte auf dem Mietwohnungsmarkt, dem Wohnungsbestandsmarkt und dem Markt für Bauland formulieren, die - gemäß den Aussagen des zweiten Hauptsatzes der Wohlfahrtsökonomik - zugleich auch notwendige Bedingungen für die Existenz eines Wohlfahrtsoptimums sind. 26)

Wie einleitend schon erwähnt wurde, handelt es sich bei den Preispfaden für den Mietzins $\{m\}_{t_{0}}^{\infty}$ und den Bodenpreis $\left\{\mathrm{P}_{\mathrm{B}}\right\}_{t_{0}}^{\infty}$ um modellendogene Variablen - obgleich sie als exogene Daten in die individuellen Optimierungskalküle eingehen -, die sich so einspielen, daß die pläne sämtlicher Marktteilnehmer zu jedem zeitpunkt miteinander kompatibel sind. Diese Kompatibilität ist in formaler Hinsicht sichergestellt, wenn $\mathrm{zu}$ jedem Zeitpunkt die Optimalitätsbedingungen der individuellen Planungsprobleme erfüllt und die betreffenden Märkte geräumt sind.

Im einzelnen bedeutet dies, daß zu jedem Zeitpunkt die Nachfrage nach Wohnleistungen dem Angebot an Wohnleistungen gleich ist - dies wird dann der Fall sein, wenn sich der Mietzins gemäß der Gleichung

$$
m=m(a / H)
$$

einspielt -, daß der Bodenmarkt geräumt ist und folglich

$$
F^{d}=F^{a}
$$

26) Daß sich das Gleichgewicht, das aufgrund der von einem zentralen Planer gefällten Entscheidungen zustande karmt, ebenfalls durch die im folgenden aufgeführten Bedingungen beschreiben läßt, zeigt SINN (1984). 
gilt und daß die folgenden Optimalitätsbedingungen $z u$ jedem zeitpunkt simultan erfüllt sind:

(II.54)

$$
\mathrm{z}\left\{\sum _ { < j } \mathrm { P } _ { \mathrm { H } } \Rightarrow \mathrm { E } \left\{\begin{array}{l}
=0 \\
\geq 0 \\
=a \delta \mathrm{H}
\end{array}\right.\right.
$$

(II.55)

$$
P_{H^{-}} \varphi^{\prime}(\varepsilon)=1
$$

für $t \geq t_{0}$,

(II.56)

$$
\frac{B}{\alpha} \varepsilon\left\{\begin{array}{l}
<\} \\
=j
\end{array} P_{B} \Rightarrow F\left\{\begin{array}{l}
= \\
\geq
\end{array}\right\} 0\right.
$$

für $t \geq t_{0}$,

$$
\hat{\mathrm{P}}_{\mathrm{H}}=-\frac{\mathrm{m}}{\overline{\mathrm{P}}_{\mathrm{H}}}+r+a \delta
$$

für $t \geq t *$,

(II.58)

$$
\hat{\mathrm{P}}_{\mathrm{H}}=\hat{\mathrm{P}}_{\mathrm{B}}=0
$$

für $t_{0} \leq t \leq t *$,

$$
\hat{\mathrm{p}}_{\mathrm{B}}=r
$$

für $t \geq t^{*}$,

(II.60)

(II.61)

$$
\lim _{t \rightarrow \infty}\left[P_{H}(t) H(t) \exp \left(-z r\left(t-t_{0}\right)\right)\right]=0,
$$

$$
\lim _{t \rightarrow \infty}\left[P_{B}(t) B(t) \exp \left(-\operatorname{zr}\left(t-t_{0}\right)\right)\right]=0 .
$$

Im Hinblick auf die Zielsetzung unserer Analyse ist es wichtig zu wissen, daß die Bedingungen (II.52) bis (II.61) keineswegs nur eine Menge statischer Gleichgewichtszustände, sondern auch einen intertemporalen Gleichgewichtspfad beschreiben. Die zeitliche Entwicklung der Nachfrage nach Wohnleistungen, die sich in einem permanenten Anstieg des Verschiebungsparameters a niederschlägt, ist hierbei die Kraft, die unsere Modellökonomie auf diesem pfad vorantreibt.

Es ist für die Zwecke unserer Analyse unerläßlich zu überprüfen, welche dynamischen Eigenschaften das in (II.52) bis (II.61) beschriebene Allokationsoptimum besitzt. Nur wenn wir wissen, wie sich Häuser- und Bodenpreise, Wohnflächen- und Baulandbestände im Zeitablauf entwickeln sollen, lassen sich auch normative Urteile über einzelne steuerliche Regelungen fällen. 
4. Die Dynamik der Modellökonomie

Es steht $z u$ erwarten, daß die Entwicklung der Nachfrage nach Wohnleistungen unmittelbar Einfluß nimmt auf die zeitliche Entwicklung des Mietzinses $m$ und des Häuserpreises $\mathrm{P}_{\mathrm{H}}$; denn bei $P_{H}$ handelt es sich definitionsgemäß um den Barwert der durch eine marginale Änderung des Wohnflächenbestandes zusätzlich erzielbaren Nettomieterträge [ vgl. Beziehung (II.26)].

Durch Differentiation von (II.26) nach der Zeitvariablen $t$ und anschließender Division dieses partiellen Differentials durch $P_{H}$ erhält man als Wachstumsrate für den Häuserpreis

$$
\hat{\mathrm{P}}_{\mathrm{H}}=\frac{\hat{\mathrm{a}}-\hat{\mathrm{H}}}{\eta} \text {, }
$$

mit $n=$ konstant als Absolutwert der Preiselastizität der Wohnleistungsnachfrage ${ }^{26)}$. Die Änderungsrate des Mietzinses und damit auch die des Häuserpreises ist eine Funktion der Veränderung des Verhältnisses zwischen Nachfrage nach und Angebot an Wohnleistungen.

Wichtig fur unsere weiteren tberlegungen ist der (II.62) ebenfalls zu entnehmende sachverhalt, daß es infolge der unterstellten Irreversibilität der Bebauung eine von der Nachfragesteigerung â abhängige Obergrenze für das Wachstum des Häuserpreises gibt. Ausgehend von der in Abschnitt II.1.2. getroffenen Vereinbarung, daß der Substanzverzehr stets in vollem

26) Offensichtlich folgt aus

$$
\partial P_{H}(t) / \partial t=m^{\prime}\left(\dot{a} / H-\dot{H} a / H^{2}\right) /(r+a \delta)
$$

die Beziehung

$$
\dot{\mathrm{P}}_{\mathrm{H}} \quad=\mathrm{m}^{\prime} \mathrm{a} \text { h }[\hat{\mathrm{a}}-\hat{\mathrm{H}}] /(\mathrm{r}+\mathrm{ab}),
$$

woraus nach Division mit $\mathrm{P}_{\mathrm{H}}$

$$
\begin{aligned}
\hat{\mathrm{p}}_{\mathrm{H}} & =\frac{\mathrm{m}^{\prime} \mathrm{a}}{\mathrm{mH}}[\hat{\mathrm{a}}-\hat{\mathrm{H}}] \\
& =\frac{\hat{\mathrm{a}}-\hat{\mathrm{H}}}{\eta}, \quad \eta=\left|\frac{\mathrm{m} H}{\mathrm{~m}^{\prime} \mathrm{a}}\right|
\end{aligned}
$$

folgt. 
Umfang durch werterhaltende Maßnahmen wiederaufgeholt wird, wird diese Obergrenze offensichtlich dann erreicht, wenn es keinen Wohnungsneubau gibt, also $\hat{\mathrm{H}}=0$ gilt. In diesem Falle nämlich erfolgt der Mietwohnungsmarktausgleich infolge des vollkommen unelastischen Angebots an Wohnleistungen alleine über eine Anhebung des Mietpreisniveaus. Man erhält dann für die maximale Wachstumsrate des Häuserpreises

$$
\left[\hat{\mathrm{P}}_{\mathrm{H}}\right]^{\max }=\frac{\hat{\mathrm{a}}}{\mathrm{n}} \text {. }
$$

Dementsprechend haben die nachfragebedingt steigenden Mieten auch Auswirkungen auf die Neubauentscheidung des Wohnungsinvestors: der Optimalitätsbedingung

$$
\mathrm{P}_{\mathrm{H}^{\prime}} \varphi^{\prime}(\varepsilon)=1
$$

können wir entnehmen, daß seine Bereitschaft, die Kapitalintensität der Bebauung eines gegebenen Grundstücks zu erhöhen, mit dem Häuserpreis $\mathrm{P}_{H}$ steigt - formal gesprochen ist dies die Folge der Numéraire-Eigenschaft des Preises für Bauleistungen und der strikten Quasi-Konkavität der Produktionsfunktion. Nach einigen elementaren Umformungen von (II.55) errechnet man sich für diesen Zusammenhang die Beziehung

$$
\left(\text { II. 64) } \quad B \hat{\varepsilon}=\hat{\mathrm{P}}_{\mathrm{H}}{ }^{27}\right. \text { ) }
$$

Die Kapitalintensität der Bebauung wächst zwar mit dem Häuserpreis, wegen $0<\beta<1$ aber mit einer größeren Rate als dieser.

Steigende Mieterträge beeinflussen aber nicht nur die Uberlegungen des Wohnungsinvestors, ein Haus welcher Größe er auf einem Grundstück gegebener Fläche errichten soll, sie beeinflussen auch seine Bereitschaft, für jede Flächeneinheit des zu bebauenden Grundstücks einen höheren Preis zu entrichten.

27) Es gilt

$$
\partial \mathrm{P}_{\mathrm{H}}(t) \partial t{ }_{\mathrm{B}} \dot{\mathrm{P}}_{\mathrm{H}}=-\varphi^{\prime \prime}(\varepsilon) \dot{\varepsilon} /\left[\varphi^{\prime}(\varepsilon)\right]^{2}=-\frac{\varphi^{\prime \prime}(\varepsilon) \varepsilon}{\left[\varphi^{\prime}(\varepsilon)\right]^{2}} \hat{\varepsilon} .
$$

Infolge der in (II.1) unterstellten Substitutionselastizität der Produktionsfunktion von Eins gilt für die Elastizizät von $\varphi^{\prime}$ bezüglich $\varepsilon$

$$
\beta=-\left[\varphi^{\prime \prime}(\varepsilon) / \varphi^{\prime}(\varepsilon)\right] \varepsilon \text {. }
$$

setzt man (ii) in (i) ein und dividiert anschließend durch $P_{H}=1 / \varphi^{\prime}(\varepsilon)$, so erhält man Beziehung (II.64). 
Diesen Zusammenhang kann man der Bedingung

(II.56) $\quad \frac{\beta}{\alpha} \varepsilon\left\{\begin{array}{l}< \\ =\end{array}\right\} P_{B} \Rightarrow F\left\{\begin{array}{l}=\} \\ \geq j\end{array} 0\right.$

entnehmen: wie wir in Abschnitt II.1.3. gezeigt haben, versteckt sich hinter dem Term $\beta E / \alpha$ das Wertgrenzprodukt des Bodens bei der Produktion von Wohnflächeneinheiten. Infolge der Konstanz der partiej.len Produktionselastizitäten $\alpha$ und $\beta$ wächst dieses Wertgrenzprodukt mit der Rate $\hat{\varepsilon}$; unter zuhilfenahme der Bedingungen (II.63) und (II.64) läBt sich deshalb auch die Obergrenze für das Wachstum des Bodenwertgrenzproduktes - hinter dem sich die maximale Zahlungsbereitschaft des Wohnungsinvestors für Bauland versteckt - bestimmen. Es gilt für den Fall, daß es keinen Wohnungsneubau gibt, die Beziehung

$$
\text { (II.65) } \quad \hat{\varepsilon}^{\max }=\frac{\hat{a}}{n \beta}
$$

Es reicht nun aber keineswegs aus zu zeigen, in welcher Weise die Entwicklung der Wohnraumnachfrage den Planungskalkül des Wohnungsinvestors beeinflußt, wenn man die Frage nach der zeitlichen Entwicklung des Wohnflächenbestandes $\mathrm{H}$ und des Baulandbestandes B beantworten will. Entscheidend für diese Antwort ist nämlich auch, ob die Bodenmakler ihrerseits willens sind, die Pläne der wohnungsinvestoren durch eine entsprechende Gestaltung ihres Baubodenangebots mitzutragen.

So setzt eine permanente Wohnungsneubautätigkeit die Bereitschaft des Bodenmaklers voraus, das in $t_{0}$ zur Beauung freigegebene Bodenareal nicht sofort zum herrschenden Preis $P_{B}\left(t_{0}\right)$ je Flächeneinheit $\mathrm{zu}$ verkaufen, sondern das dann brachliegende Land zu horten. Diese Bereitschaft hat ihrerseits wiederum den Willen des Wohnungsinvestors zur Voraussetzung, einen im zeitablauf stetig zunehmenden Preis für die Flächeneinheit Bauland $\mathrm{zu}$ entrichten. Ob und, wenn $j a$, in welchem Ausmaß diese $\mathrm{zah}-$ lungsbereitschaft wächst, hängt-dies geht aus (II.65) hervor alleine von der zeitlichen Entwicklung der Nachfrage nach wohnleistungen $\mathrm{ab}$. 
Wie unschwer festzustellen ist, ist die Höhe der Wachstumsrate â für die Dynamik unserer Modellökonomie von entscheidender Bedeutung. Diese dominierende Rolle des Zeitpfades der Wohnleistungsnachfrage sollen die folgenden Uberlegungen erhellen.

Gehen wir von einer situation aus, in der zum zeitpunkt $t_{0}$, dem Beginn unseres Planungsproblems, das Wertgrenzprodukt des Bodens - die Zahlungsbereitschaft des Wohnungsinvestors für Bauland - dem von dem Bodenmakler geforderten Preis $\mathrm{P}_{B}$ entspricht. Nun seien sowohl der Wohnungsinvestor als auch der Bodenmakler darüber informiert, daß die Nachfrage nach wohnleistungen in zukunft nur relativ schwach zunehmen wird - so schwach, daß die Zahlungsbereitschaft des wohnungsinvestors für Bauland selbst bei einem völligen Stillstand des Wohnungsneubaus und einem entsprechenden Anstieg des Niveaus der Wohnungsmieten (wegen $\hat{\mathrm{H}}=0$ wächst das Bodenwertgrenzprodukt dann mit der in (II.65) beschriebenen maximalen Rate) nicht mit einer Rate wächst, deren Wert den des Kapitalmarktzinses uibertrifft: wegen $\hat{\varepsilon}=\hat{\varepsilon}^{\max } \leq \hat{\mathrm{P}}_{\mathrm{B}}=r$ und (II.65) ist dies genau dann der Fall, wenn für das Wachstum des Verschiebungsparameters a die Beschränkung

(II. 66) $\quad \hat{a} \leq n B \hat{P}_{B}=n B r$

gilt. In diesem Fall kann es für den Bodenmakler nur von Nachteil sein, wenn er $z u$ irgend einem zeitpunkt jenseits von $t_{0}$ Bauland zur Verfügung stellt und damit Wohnungsneubau ermöglicht. Denn in diesem Falle würde der erzielbare Bodenpreis zu keinem Zeitpunkt ausreichen, den Bodenmakler für die durch das Horten von Bauland erlittenen verluste zu entschädigen.

Der optimale Angebotsplan des Bodenmaklers verlangt für den Fall â $\leq n \beta r$ offensichtlich, daß der gesamte zum zeitpunkt $t_{0}$ vorhandene Bestand $B\left(t_{0}\right)$ baureifer Grundstücke in $t_{0}$ zu dem herrschenden Marktpreis $P_{B}\left(t_{0}\right)$ verkauft wird. $B\left(t_{0}\right)$ wird dann während der Kunstzeitphase $\left(t_{0}, t^{*}\right)$ mit der optimalen Kapitalintensität $\varepsilon\left(t_{0}\right)$ bebaut - ein Vorgang, der sich in realer zeit gerechnet als kurzfristiger Bauboom in $t_{0}$ interpre- 
tieren läßt. Nach $t_{0}$ wäre dagegen jede Neubautätigkeit erloschen.

In der halblogarithmischen Darstellungsweise der Abb.5 wird dieser Sachverhalt graphisch veranschaulicht:

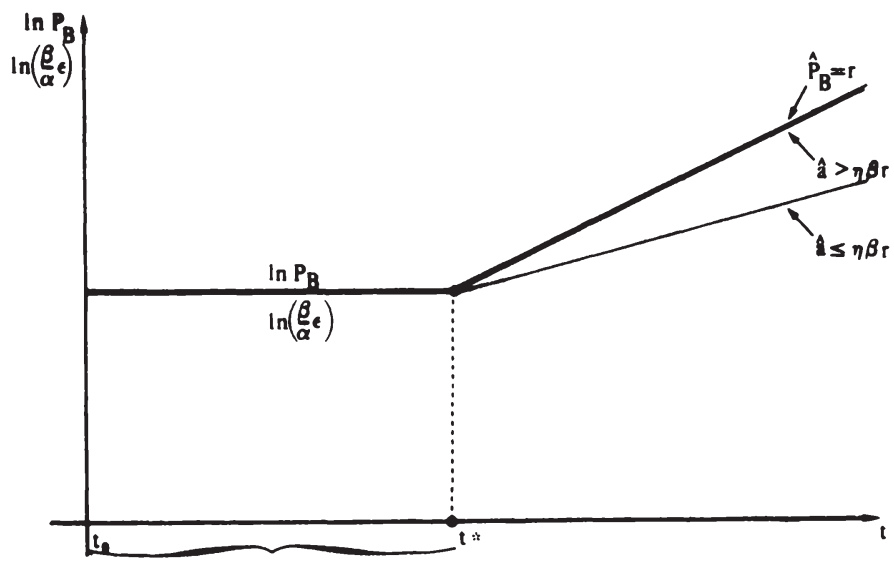

$\mathrm{Abb} .5$

KUASTELII PHASH

Die Kurven $\ln \left(P_{B}\right)$ und $\ln (\beta \varepsilon / \alpha)$ sollen die Entwicklung des gefordertem Bodenpreises bzw. des Bodenwertgrenzproduktes widerspiegeln. Da beide Größen zum Zeitpunkt $t_{0}$ annahmegemäß übereinstimmen und sich für $t_{0} \leq t<t *$ auch nicht verändern ${ }^{28}$ ), koinzidieren die logarithmierten Pfade für $P_{B}$ und $B \varepsilon / \alpha$ während der Kunstzeitphase $\left(t_{0}, t^{*}\right)$. Zwischen $t_{0}$ und $t *$ gilt in Bedingung (II.56) deshalb stets das Gleichheitszeichen, ein Umstand, der einen Verkauf von baureifem Grund und Boden ( $F>0)$, verbunden mit dem Bau neuer Wohnungen während der Kunstzeitphase impliziert. Nach Ablauf der Kunstzeitphase laufen beide Pfade dagegen stetig auseinander. In Bedingung (II.56) besitzt deshalb zu jedem Zeitpunkt jenseits von $t *$ das Ungleichheitszeichen Gültigkeit; wir werden jenseits von $t *$ deshalb keine Baulandtransaktionen und folglich auch keine Wohnungsneubau-

28) Aus (II.58) folgt $\hat{\mathrm{P}}_{\mathrm{B}}=0$, aus (II.57) in Verbindung mit (II.64) $\hat{\varepsilon}=0$. 
tätigkeit beobachten können .

Die Behauptung, daß während der Kunstzeitphase der gesamte in $t_{0}$ verfügbare Baulandbestand bebaut wird, wird durch unsere Modellalgebra bestätigt: wäre dies nämlich nicht der Fall, so würde, da sich ein Verkauf jenseits von $t *$ nicht lohnt, $\lim B(t)>0$ gelten. Da zudem $t \rightarrow \infty$

(II.59)

$$
\hat{\mathrm{P}}_{\mathrm{B}}=r
$$

gilt, könnte die Transversalitätsbedingung (II.61) in dieser Situation nur dann erfüllt sein, wenn der Bodenpreis zum Ausgangszeitpunkt $t_{\circ}$ gleich Null wäre; da andererseits aber der Wohnflächenbestand $\mathrm{H}$ und infolgedessen auch der Häuserpreis einen endichen und positiven Wert annehmen, müssen - dies verlangt die Marginalbedingung (II.55) - in $t_{0}$ auch die Kapitalintensität der Bebauung $(\varepsilon)$ und folglich auch das wertgrenzprodukt des Bodens bei der Produktion von Wohnflächeneinheiten $(B \varepsilon / \alpha)$ einen positiven endlichen wert besitzen. Die Möglichkeit, daß während der Kunstzeitphase nicht der gesamte Baulandbestand B bebaut wird, würde demnach implizieren, daß das Wertgrenzprodukt des Bodens - die Zahlungsbereitschaft des Wohnungsinvestors für die Flächeneinheit Bauland - im Zeitpunkt $t_{0}$ den von den Bodenmaklern geforderten Bodenpreis übertrifft. Dies würde jedoch die Existenzbedingung für die Lösung des Maximierungsproblems des Wohnungsinvestors verletzen; durch den kostenlosen Erwerb von Bauboden und dessen anschließende Bebauung wäre es ihm in dieser Situation nämlich möglich, den Marktwert seines Unternehmens noch zu steigern.

Wohnungsneubau jenseits von $t^{*}$ wird es im Optimum also nur dann geben, wenn die Wachstumsrate der Wohnleistungsnachfrage die Bedingung

$$
\hat{\mathrm{a}}>n \beta \hat{\mathrm{P}}_{\mathrm{B}}=n \beta r
$$


erfüllt und demgemäß $\hat{\varepsilon}^{\max } \hat{p}_{B}$ gilt. In diesem Falle verlaufen die Pfade für den geforderten Bodenpreis $P_{B}$ und das Bodenwertgrenzprodukt deckungsgleich über den gesamten Planungshorizont $\left(t_{0}, t^{*}\right)$ hinweg.

$D a \beta$ die bej.den Pfade $\ln \left(P_{B}\right)$ und $\ln (\beta \varepsilon / \alpha)$ jenseits von $t^{*}$ nicht auseinanderfallen dürfen, läßt sich unter Zuhilfenahme der ermittelten optimalitätsbedigungen begründen: der Fall, in dem der Pfad des Bodenwertgrenzprodukts jenen des Angebotspreises $P_{B}$ überschreitet, ist - wenn die Bedingungen (II.51) bis (II.61) tatsächlich die Lösung des Maximierungsproblems aus (II.19) beschreiben sollen - ausgeschlossen, da andernfalls der Wohnungsinvestor in der Lage wäre, durch eine Erweiterung seines Neubauvolumens den Marktwert seines Unternehmens zu steigern. Ebensowenig denkbar ist eine Abweichung dieses Pfades nach unten; in diesem Fall würde nämlich sofort jeder Bodenverkauf eingestelit werden - aus $\beta \varepsilon / \alpha<\mathrm{P}_{B}$ folgt gemäß (II.56) $\mathrm{F}=0$ und der Wohnflächenbestand würde stagnieren; aus $\hat{\mathrm{H}}=\mathrm{O}$ resultiert aber wiederum $\hat{\varepsilon}=\hat{\varepsilon}^{\max }>\hat{\mathrm{p}}_{\mathrm{B}}=r$ : selbst die geringste $\mathrm{Ab}-$ weichung des Bodenwertgrenzproduktes unter den Bodenpreis würde durch eine Beschleunigung des Wachstums des Bodenwertgrenzproduktes sofort wieder neutralisiert werden.

Für den Fall, daß die Nachfrage nach Wohnleistungen relativ stark wächst ( $\hat{a}>n \beta \hat{P}_{B}$ ), ist folglich fortwährend die Bedingung $\beta \varepsilon / \alpha=P_{B}$ für eine innere Lösung bezüglich des optimalen Bodenverbrauchs erfüllt; iber den gesamten Planungshorizont hinweg wird man Bodenverkäufe ebenso beobachten können wie den Bau neuer Wohnungen.

$\mathrm{DaB}$ in jeder Periode jenseits von t* Bauland verkauft wird und Wohnungsneubau stattfindet, impliziert freilich, daß der Baubodenbestand in endlicher zeit nicht restlos bebaut werden darf. Tatsächlich ist jede andere Lösung ausgeschlossen: angenommen, es sei optimal, den Bestand. B bis zu einem Zeitpunkt $t * *(t * \leq t * *<\infty)$ vollständig $z u$ bebauen. Dann könnte nach $t^{*} *$ kein Wohnungsneubau mehr stattfinden, und es würde (wegen $\hat{\mathrm{H}}=0$ ) $\hat{\varepsilon}=\hat{\varepsilon}^{\max }>\hat{\mathrm{P}}_{\mathrm{B}}$ gelten; nach $t * *$ würde das Bodenwertgrenzprodukt folglich den von den Bodenmaklern geforderten Preis $\mathrm{P}_{\mathrm{B}}$ über- 
treffen, ein Sachverhalt, der der Existenzbedingung (II.56) für ein Maximum der Hamiltonfunktion aus dem planungsproblem des Wohnungsinvestors abermals zuwiderliefe.

Die vorangegangenen Ausführungen beziehen sich auf eine Situation, in der anfänglich die Zahlungsbereitschaft des Wohnungsinvestors für Bauboden den Preisforderungen des Bodenmaklers entspricht. Eine solche Konstellation ist jedoch rein zufällig. Ebenso denkbar ist der Fall, in dem das Bodenwertgrenzprodukt zum Ausgangszeitpunkt geringer ist als der Angebotspreis $\mathrm{P}_{\mathrm{B}}$.

Auch in dieser Situation wird die Antwort auf die Frage, ob jenseits von $t *$ Bodentransaktionen und Wohnungsneubau stattfinden, im wesentlichen von der Entwicklung der Nachfrage nach Wohnungsnutzungen abhängen: auf den ersten Blick 1st man versucht zu glauben, daB in einer situation, in der die Nachfrager nach Bauboden nicht bereit sind, den von den Anbietern von Bauboden geforderten Preis zu entrichten, ein Verkauf von Bauboden jenselts aller Erwägungen des repräsentativen Bodenmaklers liegt. Doch dieser Eindruck täuscht. Wenn nämlich die Nachfrage nach Wohnleistungen nur relativ schwach ansteigt es gilt dann wieder

\section{(II.66) $\hat{\mathbf{a}} \leq n \beta r$}

- und deshalb die Steigerungsrate des Bodenwertgrenzprodukts selbst bei einem rigorosen Zurückhalten von Bauland die Bodenprelssteigerungsrate $\hat{\mathrm{P}}_{\mathrm{B}}=\mathrm{r}$ nicht übertrifft, ist es für den Bodenmakler allemal lohnender, seinen gesamten Baulandbestand zu einem Preis von

$$
P_{B}^{\prime}\left(t_{0}\right)=\frac{B}{\alpha} \varepsilon\left(t_{0}\right)<P_{B}\left(t_{0}\right)
$$

je Flächeneinheit in der Ausgangsperiode $t_{0} z u$ verkaufen, als den Verkauf hinauszuzögern und so die Schere zwischen gewünschtem und tatsächlich erzielbaren Verkaufspreis - wie in $\mathrm{Abb} .6 \mathrm{a}$ gezeigt - noch weiter auseinanderklaffen zu lassen. 
$\mathrm{Abb} .6 \mathrm{a})$

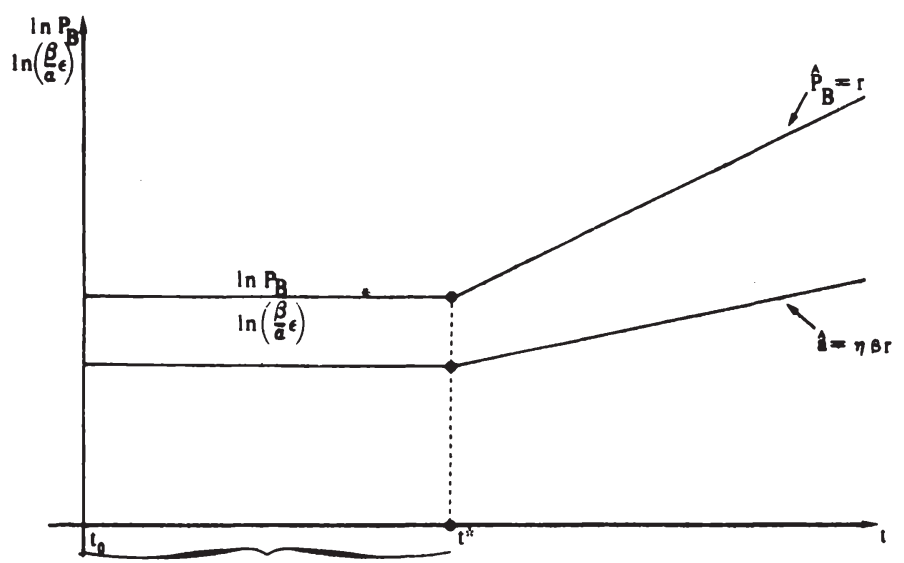

K U N S T Z E I T P H A S E

Auch in diesem Fall des schwachen Nachfragewachstums kommt fur eine Wohnungsneubautätigke1t folglich nur die Kunstzeltphase $\left(t_{0}, t^{*}\right.$ ) in Betracht, in deren Verlauf der gesamte in $t_{0}$ verfugbare Baulandbestand mit der Kapitalintensität $\varepsilon\left(t_{0}\right)$ bebaut wird (die formale Begrundung entspricht der fur den Fall $\left[P_{B}\left(t_{0}\right)=B E\left(t_{0}\right) / \alpha, \hat{a} \leq n B r\right]$ vorgebrachten). Als unmittelbare Folge der Baulandfreigabe werden wir abermals einen kurzfristigen Bauboom beobachten können, nach dessen Ende man vergeblich auf eine weitere Neubautätigkeit hoffen wird. Wohnungsbauinvestitionen treten nach $t^{*}$ dann nur noch in Form werterhaltender Maßnahmen auf.

Wenn im Ausgangspunkt unseres Planungsproblems das Wertgrenzprodukt des Bodens geringer ist als der opportunitatskostendekkende Bodenpreis $P_{B}$, so wird es nach $t^{*}$ offenbar nur dann zu einer Neubautätigkeit kommen, wenn die Bodenmakler Grund zu der Annahme haben, daß die Wohnungsinvestoren $z u$ einem absehbaren Zeitpunkt $t^{* *}>t^{*}$ bereit sein werden, den dann geforderten BOdenpreis $\mathrm{P}_{B}\left(t^{* *}\right)=\mathrm{P}_{B}\left(t^{*}\right) \exp \left[\mathrm{r}\left(t^{* *}-t^{*}\right)\right]$ zu bezahlen. 


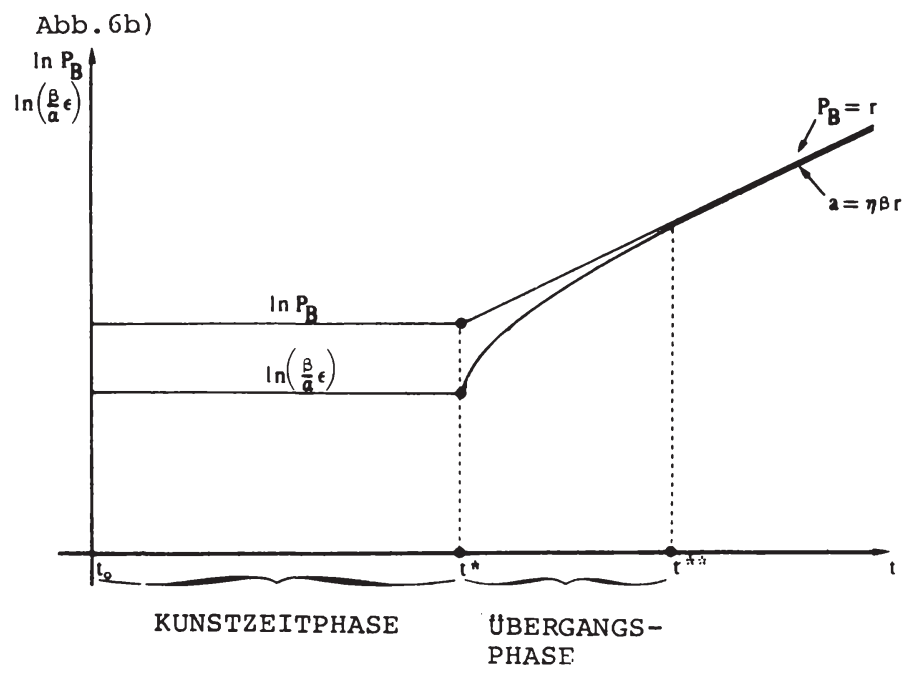

In $\mathrm{Abb} .6 \mathrm{~b}$ sind die Bedingungen für den Eintritt einer solchen Situation graphisch festgehalten. Als Folge der durch Bedingung (II.58) geforderten Konstanz aller Preise zwischen $t_{0}$ una $t *$ verlaufen die Pfade für den Bodenpreis und das Bodenwertgrenzprodukt innerhalb der Kunstzeitphase horizontal, wobei der Pfad des Bodenpreises annahmegemäß über dem des Bodenwertgrenzproduktes liegt. Voraussetzung dafür, daß sich beide Pfade in endicher Zeit treffen, ist, daß der Pfad des Bodenwertgrenzprodukts während einer Übergangaphase steiler verläuft als der des Bodenpreises. Da während dieser Ubbergangsphase wegen $B \varepsilon / \alpha<P_{B}$ keine Bodentransaktionen stattfinden und deshalb auch der Wohnungsneubau stillsteht, nimmt die steigerungsrate des Bodenwertgrenzprodukts dort den für sie maximal möglichen Wert an. Aus den Bedingungen $\hat{\varepsilon}=\hat{\varepsilon}^{\max } \hat{\mathrm{P}}_{\mathrm{B}}=\mathrm{r}$ und (II.65) folgt, daB die Nachfrage nach Wohnleistungen in diesem Fall mit einer Rate wachsen muß, die der Beschränkung
(II. 67)
$\hat{\mathrm{a}}>n \beta \mathrm{r}$

genügt. 
In unserer Graphik treffen sich die beiden Pfade zum Zeitpunkt $t * *$, jenseits von $t * *$ verlaufen sie deckungsgleich: der Fall, daß auch nach $t * *$ das Bodenwertgrenzprodukt schneller wächst als der Bodenpreis, ist ausgeschlossen, weil dadurch wiederum die Existenzbedingung für ein Maximum der Hamiltonfunktion aus dem Planunsproblem des Wohnungsinvestors verletzt werden würde. Ebensowenig denkbar ist ein Abweichen des $\beta \varepsilon / \alpha-$ Pfades nach unten: sobald die Wohnungsinvestoren nicht mehr bereit sind, den von den Bodenmaklern geforderten Preis $\mathrm{P}_{B} \mathrm{zu}$ bezahlen, werden letztere den Verkauf baureifer Grundstücke einstellen. Die Steigerungsrate des Bodenwertgrenzprodukts nimmt dann wie in der Übergangsphase $(t *, t * *)$ ihren maximalen Wert $\hat{\varepsilon}^{\max }$ an, mit der Folge, daß der Pfad des Bodenwertgrenzproduktes wieder dem $\mathrm{P}_{\mathrm{B}}$-Pfad zustrebt - selbst die kleinste denkbare Abweichung nach unten würde durch die daraufhin einsetzende Beschleunigung der Zunahme des Bodenwertgrenzprodukts wieder beseitigt werden; Bodenangebot und Bodennachfrage spielen sich so ein, daß die Wohnungsinvestoren $\mathrm{zu}$ jedem Zeitpunkt jenseits von $t * *$ bereit sind, den von den Bodenmaklern geforderten Preis $P_{B} z u$ entrichten. Es wird deshalb $z u$ jedem Zeitpunkt nach $t * *$ zu Transaktionen mit baureifen Grundstücken kommen. Dies impliziert wiederum, daß der Bestand an baureifen Grundstücken in endlicher zeit nicht vollständig aufgebraucht wird. In formaler Hinsicht bestätigt diese Uberlegung die optimalitätsbedingung (II.56) in Verbindung mit den Optimalitätsbedingungen (II.55) und (II.61).

Aus Gründen der tbersichtlichkeit empfiehlt es sich, an dieser Stelle ein kurzes Fazit aus unseren bisherigen Uberlegungen zu ziehen:

Eine permanente Wohnungsneubautätigkeit kann es nur dann geben, wenn das Wachstum der Nachfrage nach Wohnleistungen stark genug ist, bei einem konstanten wohnflächenbestand dafür zu sorgen, daß die wachstumsrate des Bodenwertgrenzprodukts $\ddot{u}$ b e r den opportunitätskosten des Hortens von Bauland liegt. Der paretooptimale Entwicklungspfad der Laisser-Faire-ökonomie ist dann dadurch gekennzeichnet, daB nach einer möglichen Anpassungsphase 
der Wohnflächenbestand $H$, der Häuserpreis $P_{H}$, der Mietzins $m$, der Bodenpreis $\mathrm{P}_{\mathrm{B}}$ sowie die Kapitalintensität der Bebauung $\varepsilon$ im Zeitablauf allmählich ansteigen. Der Bestand an baureifen Grundstücken B schrumpft zwar stetig im Laufe der zeit, wird aber in endlicher zeit nicht vollständig bebaut, sondern geht erst für $t \rightarrow \infty$ gegen Null.

Schon dieses vorläufige Ergebnis unserer Analyse liefert uns erste Kriterien für die Beurteilung gegebener Wohnungspolitiken: zumindest im Falle einer relativ stark ansteigenden Nachfrage nach Wohnleistungen wäre eine Politik, die auf eine möglichst schnelle Bebauung baureifer Grundstücke drängt, aus Gründen der gesellschaftlichen Wohlfahrt suboptimal; bei der Frage, ob die Eigentümer baureifer Grundstücke durch entsprechende statiche Eingriffe veranlaBt werden sollten, Bauland in stärkerem Maße als bisher zur verfügung zu stellen, darf nicht allein von der aktuellen Nachfragesituation am (Miet-) wohnungsmarkt ausgegangen werden, es müssen vielmehr auch zukünftige Entwicklungen in die Uberlegungen miteinbezogen werden. Nur wenn die steigerungsrate der Wohnleistungsnachfrage vergleichsweise mäBig ist, läBt sich die Existenz von Baulucken unter wohlfahrtstheoretischen Aspekten nicht mehr rechtfertigen.

Der Fall des mäßigen Nachfragewachstums ist für unseren weiteren Analyseweg allerdings weniger interessant. Wir wollen uns im folgenden der Betrachtung einer okonomie widmen, in der die Nachfrage nach Wohnungsnutzungen die Wachstumsbedingung

$$
\hat{a}>n \beta \hat{P}_{B}=n \beta r
$$

erfullt. Wie wir wissen, sind damit auch - zumindest nach dem Ablauf einer denkbaren Ubergangsphase $\left(t *, t^{* *}\right)$ - stets die Bedingungen

$$
\begin{array}{ll}
\text { (II.69) } & \frac{\beta}{\alpha} \varepsilon=\mathrm{P}_{B}, \\
(I I .70) & \hat{\varepsilon}=\hat{P}_{B}
\end{array}
$$


für einen permanenten Wohnungsneubau erfüllt.

Setzt man (II.70) unter Berücksichtigung von

(II.64)

$$
\hat{\mathrm{P}}_{\mathrm{H}}=\beta \hat{\varepsilon}
$$

in die in (II.62) gefundene Beziehung für die Wachstumsrate des Häuserpreises ein, so erhält man nach einer einfachen Umformung für die Wachstumsrate des Wohnflächenbestandes

$$
\hat{\mathrm{H}}=\hat{\mathrm{a}}-n \beta \hat{\mathrm{P}}_{\mathrm{B}^{-}}=\text {konstant }>0 \text {. }
$$

Gleichung (II.71) bestätigt nur unsere vorangegangenen Uberlegungen: ist die Anderung in der Nachfrage nach Wohnleistungen relativ stark ( $\hat{a}>n \beta \hat{\mathrm{P}}_{B}$ ), so wird in jeder Periode neuer wohnraum errichtet und der Wohnflächenbestand wachsen.

Aus der Wachstumsgleichung (II.71) lassen sich auch Aussagen über die Entwicklung des Baubodenbestandes ableiten. Da, wie aus (II.71) unmittelbar hervorgeht, die wachstumsrate $\hat{H}$ eine Konstante ist, gilt folgerichtig $\hat{\mathrm{H}}=\hat{\mathrm{H}}$. Da zudem der Marktwert einer Wohnflächeneinheit annahmegemäB den Wert einer D-Mark ubertrifft und deshalb der gesamte abnutzungsbedingte substanzverzehr des Gebäudekapitalstocks in jeder Periode durch werterhaltende Arbeiten kompensiert wird, errechnet man sich aus der Bewegungsgleichung

$$
\text { (II.10) } \quad \dot{\mathrm{H}}=\mathrm{F} \varphi(\varepsilon)-z(\alpha \delta \mathrm{H}-E)
$$

wegen $\alpha \delta \mathrm{H}=\mathrm{E}$ und (II.37) für die Wachstumsrate des :Wohnflächenbestandes die Gleichung

$$
\hat{\dot{H}}=\hat{H}=\hat{\varphi}+\hat{F}=\alpha \hat{E}+\hat{F} \text {. }
$$

Substituiert man (II.72) in (II.71), so erhält man als Ergebnis die Wachstumsrate des Bodenverbrauchs: 
(II.73)

$$
\left.\hat{F}=\hat{a}-\hat{P}_{B}(n \beta+\alpha)=\text { konstant }<0.29\right)
$$

Nun lautet die Bewegungsgleichung des Bodenbestandes $\dot{B}=-F$. Berücksichtigt man darüberhinaus noch, $\mathrm{da} B \mathrm{dF} / \mathrm{dB}=\dot{\mathrm{F}} / \dot{\mathrm{B}}$, so gilt (II.74) $\quad \frac{\mathrm{dF}}{\mathrm{d} B}=-\frac{\dot{\mathrm{F}}}{\mathrm{F}}=-\hat{\mathrm{F}}=$ konstant $>0$.

Der Bodenverbrauch ist demnach eine lineare Funktion des Bodenbestandes, die sich allgemein in der Form

$$
F=b_{1}+b_{2} B, b_{1}, b_{2}>0
$$

darstellen läßt; wie sich aber schnell zeigt, muß in (II.75) $\mathrm{b}_{1}=0$ gelten: wäre $\mathrm{b}_{1}$ größer als Null, würde der Baulandbestand in endlicher Zeit bebaut werden. Zwangsläufige Folge dieses Bodenverkaufsplans wäre, daß das Wertgrenzprodukt des Bodens den Bodenpreis in endlicher Zeit übertrifft, ein Umstand, der mit der Optimalitätsbedingung (II.56) aus dem Planungproblem des Wohnungsinvestors nicht $\mathrm{zu}$ vereinbaren ist. Wäre $b_{1}$ kleiner als Null, würde ein Teil des Bodens niemals bebaut werden. Auch dieser Angebotsplan des Bodenmaklers wird durch die Modellalgebra, speziell durch die Optimalitätsbedingung (II.56) in Verbindung mit der Transversalitätsbedingung (II.61), ausgeschlossen.

Aus $b_{1}=0$ folgt dann $\hat{F}=\hat{B}$ bzw.

$$
\hat{\mathrm{B}}=\hat{\mathrm{a}}-\hat{\mathrm{P}}_{\mathrm{B}}(\eta \beta+\alpha)=\text { konstant }<0 \text {. }
$$

Mit Hilfe der Wachstumsgleichungen (II.71) und (II.76) lassen sich die dynamischen Eigenschaften der Zustandsvariablen $\mathrm{H}$ und $\mathrm{B}$

29) $\mathrm{DaB}$ die Wachstumsrate des Bodenverbrauchs negativ ist, folgt aus der Transversalitätsbedingung für die Zustandsvariable H: (II.61) fordert offensichtlich

(i) $\hat{\mathrm{H}}+\hat{\mathrm{P}}_{\mathrm{H}}-\mathrm{r}<0$.

Setzt man (i) in (II.71) ein und beachtet die Tatsache, daß der Zinssatz gemäß der in (II.59) formulierten HOTELIING-Regel mit der wachstumsrate des Bodenpreises ibbereinstimmt, erhält man das negative Vorzeichen für die Veränderungsrate des Bodenverbrauchs. 
auch graphisch hervorheben; denn offensichtlich beschreiben diese beiden Gleichungen in einem ( $H, B)$-Diagramm eine Schar isoelastischer Kurven. Es gilt

$$
\frac{\mathrm{dH}}{\mathrm{dB}}=\frac{\dot{\mathrm{H}}}{\dot{\mathrm{B}}}=\frac{\mathrm{H}\left[\hat{\mathrm{a}}-n \beta \hat{\mathrm{P}}_{\mathrm{B}}\right]}{\mathrm{B}\left[\hat{\mathrm{a}}-(n \beta+\alpha) \hat{\mathrm{P}}_{\mathrm{B}}\right]}<0 .
$$

Als vier Vertreter dieser Schar wurden in den rechten Quadranten von $\mathrm{Abb} .7$ die Hyperbeln AA, CC, DD und EE eingezeichnet.

$\mathrm{Abb} .7$

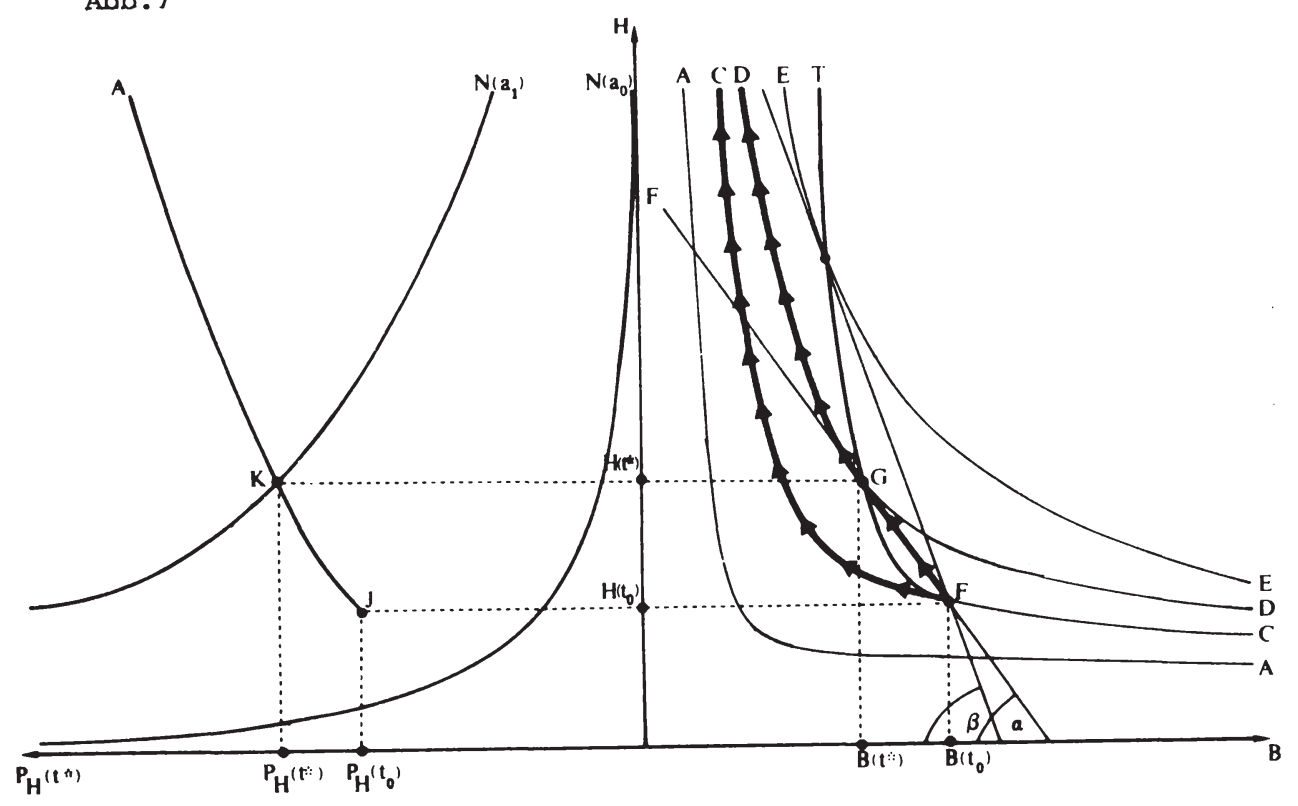

Auf einer dieser (unendlich vielen) Kurven müssen sich Wohnflächen- und Baubodenbestand in realer Zeit bewegen, wenn - wie wir dies in unserer Analyse unterstellt haben - zumindest jenseits eines endlichen Zeitpunktes $t^{* *}$ in jeder Periode neue Wohnungen gebaut werden. Welcher Kurve hierbei zunächst unser spezielles Interesse zu gelten hat, hängt von den historischen Anfangsbedingungen $\mathrm{H}\left(t_{0}\right)$ und $\mathrm{B}\left(t_{0}\right)$ ab - in $\mathrm{Abb} .7$ ist dies die 
Hyperbel CC. Punkt $\mathrm{F}$ ist der graphische Startpunkt unserer Modellökonomie.

Liegt zum Zeitpunkt $t_{0}$ der geforderte Bodenpreis $\mathrm{P}_{\mathrm{B}}$ über der Zahlungsbereitschaft $\beta E / \alpha$ der Wohnungsinvestoren, so wissen wir aus unseren vorangegangenen Uberlegungen, daß während einer tbergangaphase $\left(t^{*}, t^{* *}\right)$ keine Baulandverkäufe stattfinden und deshalb auch der Wohnungsneubau stillsteht. Das System verharrt wăhrend dieser Ubergangsphase folglich in Punkt $F$, um sich jenseits von $t * *$ entlang der Kurve CC in Pfeilrichtung zu bewegen.

Die Steigung des Entwicklungspfades informiert uns gleichzeitig auch darüber, wie sich im Zuge dieses Prozesses die Kapitalintensität der Bebauung verändert; aus (II.10) und (II.45) folgt wegen $E=\alpha \delta \mathrm{H}$ die Beziehung

$$
\frac{\mathrm{dH}}{\mathrm{dB}}=\frac{\dot{\mathrm{H}}}{\dot{\mathrm{B}}}=-\varphi(\varepsilon) .
$$

Je steller der Entwicklungspfad verläuft, d.h. je weniger noch nicht bebautes Bauland zur Verfügung steht, desto größer werden die Häuser, mit denen Grundstücke gegebener Fläche bebaut werden.

Es ist nun aber keineswegs so, daß sich das system, ausgehend von F, immer und in jedem Fall entlang des isoelastischen Pfades CC entwickeln wird. Wenn nämlich in $t_{0}$ Bodenwertgrenzprodukt und Bodenpreis übereinstimmen, werden - dies wurde in Abb.5 gezeigt - schon im Verlauf der Kunstzeitphase ( $\left.t_{0}, t^{*}\right)$ neue Wohnungen gebaut; in realer zeit gerechnet findet eine sprunghafte Anpassung des Wohnflächenbestandes nach oben statt. Entsprechend unstetig nimmt der Bestand baureifer Grundstücke ab. Bei der graphischen Beschreibung dieser Sprünge in den Zustandsvariablen erweist sich die Information aus Gleichung (II.78) als ebenso hilfreich wie die Kenntnis der Bestimmungsfaktoren der Variablen $\varepsilon$ und deren Entwicklung innerhalb der Kunstzeitphase $\left(t_{0}, t^{*}\right)$ : ein Blick auf die Bedingung (II.56) zeigt uns, daß der Wohnungsinvestor die Entscheidung, ein Haus welcher Größe er auf einem Grundstück gegebener Fläche errichten 
soll, vom Verhältnis zwischen dem Preis $P_{B}$ für eine Flächeneinheit Bauland und dem preis $P_{I}$ für jede Einheit Bauleistungen (mit $P_{I}=1$ ) abhängig macht. Und Bedingung (II.58) sagt uns, daß sich diese Preisrelation in der Kunstzeitphase nicht verändert; innerhalb von $\left(t_{0}, t^{*}\right)$ behalten damit aber auch die Kapitalintensität $\varepsilon\left(t_{0}\right)$ und mit ihr die marginale Bebauungsdichte $\varphi\left[\varepsilon\left(t_{0}\right)\right]$ ihren Ausgangswert bei. Der Entwicklungspfad für die Zustandsvariablen $H$ und $B$ stellt sich in termini unseres $(H, B)$ - Diagramms aus Abb. 7 wischen $t_{0}$ und $t^{*}$ somit als eine Gerade dar - dies geht aus (II.78) hervor -, die ausgehend von $F$ nach links oben hin ansteigt; in Abb.7 ist dies die Linie $F F$, deren Steigungsma $B$ identisch ist mit dem Tangens des winkels $\alpha$, für den wiederum $\tan \alpha=-\varphi\left[\varepsilon\left(t_{0}\right)\right]$ gilt.

Man kann Abb.7 auch die "Dauer" der Kunstzeitphase ( $\left.t_{0}, t^{*}\right)$ entnehmen. Hierzu muß man lediglich wissen, daß eine in der Kontrolltheorie zwingende Bedingung die Kontinuität u.a. auch der Zeitpfade der (Schatten-) Preise verlangt. Dies wiederum impliziert, daß es beim Ubergang von der künstlichen auf die reale Zeit keinen Knick im Entwicklungspfad geben darf - ein solcher Knick würde wegen (II.78) einen Sprung in $\varepsilon$ und wegen der optimalitätsbedingungen (II.55) und (II.56) auch einen sprung in den Preisen $P_{H}$ und $P_{B}$ implizieren. Demnach wird das system die Gerade FF exakt in jenem Punkt verlassen - ist die Kunstzeitphase exakt zu dem zeitpunkt beendet-, in dem die Gerade FF eine isoelastische Kurve tangiert. Im Diagramm der Abb.7 ist dies der Punkt $G$. In realer zeit gerechnet ist das system von seinem Ausgangszustand $\left[H\left(t_{0}\right), B\left(t_{0}\right)\right]$ in den Zustand [H(t*), $\left.B(t *)\right]$ gesprungen und entwickelt sich danach entlang des isoelastischen Pfades GD weiter.

Die graphische Konstruktion, die hier für einen bestimmten Wert $\varepsilon_{0}\left(t_{0}\right)$ der Kapitalintensität beschrieben wurde, läßt sich für das gesamte Kontinuum möglicher Werte für $\varepsilon$ wiederholen (in Abb.7 ist dies beispielhaft fur einen Wert $\varepsilon_{1}\left(t_{0}\right)>\varepsilon_{0}\left(t_{0}\right)$ angedeutet). Wenn man für jeden beliebigen wert von $\varepsilon$ den $z$ u $G$ korrespondierenden Tangentialpunkt bestimmt und diese Punkte miteinander verbindet, erhält man als Resultat die Sprungkurve FT in Abb. 7 . 
Diese Sprungkurve spielt für die graphische Erläuterung der im Rahmen des Modells abzuleitenden Ergebnisse eine wichtige Rolle. Denn sie ist der geometrische Ort aller Zustände, die als Ergebnis einer in $t_{0}$ stattfindenden Anpassungsreaktion in Frage kommen. Welcher Punkt auf FT von einem von $F$ ausgehenden strahl nun tatsächlich anvisiert wird, hängt $a b$ von der Höhe des Relativpreises $\mathrm{P}_{B}\left(t_{0}\right)$, die wiederum abhängig ist von der Zahlungsbereitschaft der Wohnungsinvestoren für die Flächeneinheit Bauland in $t_{0}$. Diese Zahlungsbereitschaft wird ihrerseits umso höher sein, je günstiger sich die Ertragslage des Wohnungsinvestors am Mietwohnungsmarkt zum zeitpunkt $t_{0}$ darstellt.

Den Zusammenhang zwischen Ertragsmöglichkeiten und Investitionsverhalten gibt der linke Quadrant der Abb.7 wieder. Auf der Abszisse dieses Quadranten ist der Häuserpreis zum Zeitpunkt $t *$ ( $\left.\equiv t_{0}\right)$ abgetragen. Die in diesem Schaubild eingezeichnete Kurve A gibt an, welchen Bestand an Wohnflächeneinheiten der Wohnungsinvestor zum Zeitpunkt $t *$ ( $t_{0}$ ) bei alternativen Mietbarwerten $\mathrm{P}_{\mathrm{H}}\left(t^{*}\right) \mathrm{zu}$ halten bereit ist. Da jede dieser Wohnflächeneinheiten definitionsgemäß in jeder Periode genau eine Wohnleistungseinheit abgibt, läßt sich $A$ auch als Angebotskurve für Wohnleistungen interpretieren. Bei der Ableitung dieser Kurve wurde dem Umstand Rechnung getragen, daß sich bei einer gedanklichen Bewegung auf der FT-Kurve - dem geometrischen ort aller zum Zeitpunkt $t *$ denkbaren optimalen Wohnflächenbestände $\mathrm{H}\left(t^{*}\right)$ - der optimale Wohnflächenbestand und die optimale Kapitalintensität der Bebauung gleichgerichtet verändern. Dies erlaubt uns die Formulierung einer funktionalen Beziehung

$$
H\left(t^{*}\right)=\Phi\left[\varphi\left(\varepsilon\left(t^{*}\right)\right)\right], \Phi^{\prime}>0 .
$$

Den kausalen Zusammenhang zwischen der Variablen $\varepsilon$ und der Ertragslage am Markt für Wohnleistungen kann man der Marginalbedingung (II.55) entnehmen, die für das Investitionsoptimum die Gleichheit des Wertgrenzprodukts der marginalen Bauleistung mit den durch diese verursachten (Grenz-) Kosten fordert. Wenn man Bedingung (II.55) invertiert und die so erhaltene Inverse in 
(II.79) substituiert, erhält man den für den zeitpunkt t* gesuchten Zusammenhang zwischen Wohnungsbestandsnachfrage bzw. Wohnleistungsangebot und Häuserpreis:

$$
H\left(t^{*}\right)=\Phi\left\{\varphi\left[\varphi^{\prime-1}\left(1 / P_{H}\left(t^{*}\right)\right)\right]\right\}
$$

Wie sich leicht zeigen läßt, gilt in (II.80)

$$
\text { (II.81) } \quad \frac{U H\left(t^{*}\right)}{U P_{H}\left(t^{*}\right)}>0
$$

Je stärker also der erwartete Barwert der Mieterträge einer Wohnflächeneinheit zunimnt, desto eher ist der Wohnungsinvestor bereit, seine Bestandsnachfrage nach Wohnflächeneinheiten bzw. sein Angebot an Wohnleistungen in $t_{0}$ auszudehnen (et vice versa).

Die Angebotskurve ist nach unten durch den Ordinatenwert $H\left(t_{0}\right)$ begrenzt: infolge der Irreversibilität der Bebauung kann das Wohnleistungsangebot $-\alpha \delta \mathrm{H}=\mathrm{E}$ vorausgesetzt - nicht unter sein anfängliches Niveau absinken.

Das zum Zeitpunkt $t *$ optimale Angebot an Wohnleistungen (und damit der optimale Bestand $\mathrm{H}\left(t^{*}\right)$ ) stellt sich als Schnittpunkt der in (II.80) beschriebenen Angebotskurve mit der Nachfragekurve $\mathrm{N}$ dar. Hinter dieser Nachfragekurve verbirgt sich die Optimalitätsbedingung

$$
\hat{\mathrm{P}}_{\mathrm{H}}=-\frac{\mathrm{m}(\mathrm{a} / \mathrm{H})}{\mathrm{P}_{\mathrm{H}}}+r+\alpha \delta
$$

Berücksichtigt man, daß wegen (II.64) und (II.70)

$$
\hat{\mathrm{P}}_{\mathrm{H}}=\boldsymbol{B} \hat{\mathrm{P}}_{\mathrm{B}}
$$

gilt, so erhält man aus (IJ.57) durch einfaches Umformen

$$
\mathrm{P}_{\mathrm{H}}=\frac{\mathrm{m}(\mathrm{a} / \mathrm{H})}{r+\alpha \delta-\beta \hat{\mathrm{P}}_{\mathrm{B}}} .
$$

(II.83) informiert uns darüber, $\mathrm{zu}$ welchem Mietzins (und damit zu welchem Häuserpreis $\mathrm{P}_{\mathrm{H}}$ ) die privaten Haushalte bereit sind, 
ein bestimmtes, von den Wohnungsinvestoren avisiertes Wohnleistungsangebot $\mathrm{H}$ zu konsumieren. Für die steigung dieser (impliziten) Preisabsatziunktion gilt offensichtlich

(II.84) $\quad \frac{\partial \mathrm{P}_{\mathrm{H}}\left(t^{*}\right)}{\partial \mathrm{H}\left(\mathrm{t}^{*}\right)}<0$.

Im linken Quadranten der $\mathrm{Abb} .7$ sind zwei Nachfragekurven eingezeichnet, die den beiden zuvor diskutierten situationen entsprechen:

- Angenommen, die Nachfragekurve $N\left(a_{0}\right)$ spiegelt die in $t_{0}$ herrschenden Nachfrageverhältnisse korrekt wider. In dieser Situation sind die privaten Haushalte nicht bereit, das vorhandene Angebot an Wohnleistungen $z u$ einem für den Wohnungsinvestor akzeptablen Mietpreis in vollem Umfang nachzufragen. Infolge der Irreversibilität der Bebauung muß der Wohnungsinvestor hinnehmen, $d a B$ er zumindest vorübergehend entweder einen Teil seines Wohnflächenbestandes nicht vermieten kann oder aber seinen gesamten Wohnflächenbestand zu einem für ihn nicht kostendekkenden Preis vermieten muß. Angesichts dieser momentan schlechter Ertragslage ist verständlicherweise seine Bereitschaft zum Bau neuer Wohnungen nur schwach ausgeprägt. Insbesondere wird er nicht bereit sein, für eine Flächeneinheit des für eine Bebauung erforderlichen Grundstücks den von dern Bodenmakler geforderten Preis zu entrichten. Da der Bodenmakler in Kenntnis der künftigen Nachfrageverhältnisse am Mietwohnungsmarkt seinerseits nicht bereit ist, einen geringeren Bodenpreis zu akzeptieren, wird es $z u$ einem vorübergehenden Stillstand im Bereich des Wohnungsneubaus kommen. Diese in $\mathrm{Abb} .6 \mathrm{~b}$ so bezeichnete Ubergangsphase $(t *, t * *)$ wird erst dann beendet sein, wenn sich die Nachfragekurve unter dem Einfluß des permaneten Nachfragewachstums soweit nach links verschoben hat, daß sie die Angebotskurve zum Zeitpunkt $t * *$ in Punkt $J$ schneidet; erst $z u$ diesem Zeitpunkt werden die Wohnungsinvestoren aufgrund des gestiegenen Niveaus der Wohnungsmieten wieder willens sein, den Angebotspreis $P_{B} z u$ entrichten. Verharrte die Modellökonomie aufgrund des fehlenden Wohnungsneubaus während dieser Ubergangs- 
phase in Punkt $F$, so wird sie sich nach $t * *$ entlang der Kurve $\mathrm{CC}$ in Pfeilrichtung weiterbewegen.

- Die Nachfragekurve $N\left(a_{1}\right)$ indiziert dagegen eine Situation, in der zum Zeitpunkt $t_{0}$ die Wohnleistungsnachfrage der Haushalte bei dem $z$ um Häuserpreis $\mathrm{P}_{\mathrm{HO}^{\mathrm{O}}}(t *)$ korrespondierenden Mietzins $m_{0}$ das Wohnleistungsangebot des Wohnungsinvestors übertrifft. In diesem Fall wird es sich für den Wohnungsinvestor lohnen, zum Zeitpunkt $t_{0}$ eine Anpassung seines Wohnflächenbestandes vorzunehmen, denn sowohl das Wertgrenzprodukt des Bodens als auch das der Bauleistungen ubertreffen in dieser Situation die Faktorpreise $\mathrm{P}_{B}$ bzw. $\mathrm{P}_{I}$. Die optimalitätsbedingungen (II.55) und (II.56) sind erst dann wieder erfüllt, wenn der Wohnflächenbestand sein optimales Niveau $H\left(t^{*}\right)$ erreicht hat. Die Bestandsanpassung von $H\left(t_{0}\right)$ auf $H\left(t^{*}\right)$ vollzieht sich in der Kunstzeitphase $\left(t_{0}, t^{*}\right)$. Das System springt hierbei von Punkt $F$ auf jenen Punkt $G$ der Sprungkurve FT, dessen H-Koordinate mit dem Optimalwert $H(t *)$ übereinstimmt. Jenseits von $t *$ wandert das System entlang jener Hyperbel, die in $G$ den linearen Pfad FF tangiert.

Mit Hilfe von $\mathrm{Abb} .7$ und des sich dahinter verbergenden Katalogs von Optimalitätsbedingungen lassen sich nun die Reaktionen des Wohnungs- und Bodenmarktes auf die steuerpolitischen Eingriffe des Staates beschreiben. Vor allem die ubergangsphase $\left(t^{*}, t^{* *}\right)$ und die Kunstzeitphase $\left(t_{0}, t^{*}\right)$ werden relevant, wenn die Entscheidungsprobleme der beiden repräsentativen Modellakteure durch nicht-antizipierte Veränderungen ihrer Planungsdaten gestört werden - dies deshalb, weil wir grundsätzlich mit zwei gegensätzlichen Typen von kurzfristigen Reaktionen rechnen müssen:

- zum einen kann eine Steuer als eine solche Belastung empfunden werden, daß der Wohnungsneubau zumindest vorübergehend zum Erliegen kommt;

- zum anderen ist es ebenso denkbar, daß es infolge steuerlicher Ungleichbehandlungen $\mathrm{zu}$ einer (kurzfristigen) Intensivierung des Wohnungsneubaus kommt. 
Beiden Kategorien von Reaktionen ist gemeinsam, daB sie den paretooptimalen Entwicklungsprozess unserer Modellökonomie beeinträchtigen:

- bei einem vorübergehenden Stillstand des Wohnungsneubaus würde das System zwar den optimalen Pfad CC nicht verlassen, es würde aber der paretooptimalen Entwicklung auf diesem Pfad hinterherhinken;

- besteht die Reaktion dagegen in einem kurzfristigen Bauboom, wird der optimale pfad zum zeitpunkt des Eintritts der störung verlassen; die weitere Entwicklung erfolgt dann entlang eines anderen, suboptimalen pfades.

Um die Mechanik dieser Reaktionen begreifen und beschreiben zu können, genügt ein Blick auf die folgenden Gleichungen respektive auf die Veränderungen, die diese Gleichungen unter dem Einfluß der Besteuerung erfahren:

$$
\begin{aligned}
& \frac{\mathrm{dH}}{\mathrm{dB}}=\frac{\dot{\mathrm{H}}}{\dot{\mathrm{B}}}=\frac{\mathrm{H}\left[\hat{\mathrm{a}}-n \beta \hat{\mathrm{P}}_{\mathrm{B}}\right]}{\mathrm{B}\left[\hat{\mathrm{a}}-(n \beta+\alpha) \hat{\mathrm{P}}_{\mathrm{B}}\right]}, \\
& \mathrm{H}\left(t^{*}\right)=\Phi\left\{\varphi\left[\varphi^{\prime-1}\left(1 / \mathrm{P}_{\mathrm{H}}\left(t^{*}\right)\right)\right]\right\},
\end{aligned}
$$

$$
P_{H}=\frac{m(a / H)}{r+\alpha \delta-\beta \hat{P}_{B}} .
$$

Kommt es infolge der Implementierung spezieller steuerarten zu einer Veränderung des Differentialquotienten $\mathrm{dH} / \mathrm{dB}$ in Gleichung (II.77), so schlägt sich dies graphisch in einer Drehung der isoelastischen Entwicklungspfade und - man erinnere sich an die Konstruktion dieser Kurve - der sprungkurve FT nieder. Darüberhinaus wird sich unter dem Einfluß einer steuerinduzierten Veränderung von $d H / d B$ auch die Lage der Angebotskurve verändern: wegen (II.78) läßt sich die Marginalbedingung (II.55) auch schreiben als

$$
\begin{aligned}
(I I .55)^{\prime} \quad \mathrm{P}_{\mathrm{H}} & =1 / \varphi^{\prime}(\varepsilon) \\
& =1 / \varphi^{\prime}\left[\varphi^{-1}(|\mathrm{dH} / \mathrm{dB}|)\right] .
\end{aligned}
$$


mit $\mathrm{dP}_{\mathrm{H}} / \mathrm{d}(|\mathrm{dH} / \mathrm{dB}|)>0$.

Bedingung (II.55)' stellt einen unmittelbaren Zusammenhang her zwischen der Steigung der isoelastischen Entwicklungspfade und dem Ordinatenabstand der Kurve des Wohnleistungsangebots: sollten die Entwicklungspfade im. AnschluB an eine modellexogene Parametervariation steiler (flacher) verlaufen, so wird der ordinatenabstand größer (kleiner) werden, die Angebotskurve also nach links (rechts) rücken.

Es liegen aber auch steuerinduzierte Verschiebungen der Angebotskurve im Bereich des Möglichen, ohne daß die isoelastischen Entwicklungspfade und die Sprungkurve FT ihre Position verändern. Voraussetzung hierfür ist, daß sich die Marginalbedingung (II.55) unter dem EinfluB der Besteuerung verändert.

Schließlich sagen uns steuerinduzierte Veränderungen der Gleichgewichtsbedingung (II.83), wie sich die Position der Nachfragekurve in $\mathrm{Abb} .7$ verändert.

Wichtig für das Verständnis der folgenden Ausführungen ist es darüberhinaus, $z \mathrm{u}$ wissen, daß sich aus Verschiebungen der Nachfrage- bzw. Angebotskurve die kurzfristigen Reaktionen der betrachteten Modellakteure auf die Einführung von Steuern ablesen lassen; der EinfluB der Besteuerung auf die langfristigen Wachstumseigenschaften der Modellökonomie schlägt sich graphisch in einer Positionsänderung der isoelastischen Entwicklungspfade und der Sprungkurve nieder.

Wir wollen uns in dem nun folgenden Kapitel mit der Frage beschäftigen, in welcher Weise die Besteuerung auf die Bedingungen (II.77), (II.80) und (II.83) EinfluB nimmt. 
I I I .

REAKTIONEN DES WOHNUNGSMARKTES UND DES MARKTES FÜR BAULAND AUF STEUERLICHE EINGRIFFE DES STAATES

Wie alle anderen Sektoren, in denen Gewinne erzielt werden und Vermögen gebunden ist, tragen auch der Wohnungs- und der Baubodensektor zur Deckung des Finanzierungsbedarfs der öffentlichen Hand bei.

Nicht zuletzt aufgrund der Tatsache aber, daß vor allem der Wohnungssektor auch ein sehr beliebtes Betätigungsfeld vermögens- und verteilungspolitisch motivierter Politiker war und ist, weist die steuerliche Behandlung des Wohnungseigentums und in ihrem Gefolge auch die des Bodeneigentums - eine solch bunte Vielfalt wenig aufeinander abgestimmter Einzelbestimmungen auf, wie sie in kaum einem anderen sektor unserer Volkswirtschaft beobachtet werden kann.

Als ob die damit gestiftete Verwirrung noch nicht groß genug wäre, wird dieses Sammelsurium durch eine Vielzahl von Erlässen diverser (Ober-)Finanzdirektionen und Urteilen der Finanzgerichtsbarkeit bereichert, die ihren Ursprung sehr oft in formal-juristischen und nur selten in ökonomischen Uberlegungen haben. Es würde die Arbeit von mehreren Jahren erfordern, wollte man diesen Dschungel von Verordnungen und Erlassen durchforsten und sich alleine darauf beschränken, die dabei zutage geförderten Widersprüche und Ungereimtheiten aufzulisten.

In diesem Sinne muß es sich bei der folgenden steuerwirkungsanalyse zwangsläufig um Stückwerk handeln; denn aus Gründen der Ubersichtlichkeit und Nachvollziehbarkeit empfiehlt es sich, von der expliziten Betrachtung verwaltungstechnischer Detailfragen abzusehen und sich auf die eigentlichen Mängel in der Besteuerung des Wohnungs- und Bodeneigentums zu konzentrieren. Schon diese grundlegende Analyse wird zeigen, an was es dem Wohnungssektor in steuerlicher Hinsicht am meisten fehlt - an einer klaren und konsistenten Konzeption, die erkennen läßt, daß der Gesetzgeber die Wichtigkeit der wohlfahrtstheoretischen Problematik erkannt und verarbeitet hat. 
1. übersicht der zu analysierenden steuerarten

Unsere Aufgabe im folgenden wird sein aufzuzeigen, wie die in Kapitel II beschriebene Modellökonomie reagiert, wenn zum Ausgangszeitpunkt $t_{0}$ bestimmte Steuern eingeführt bzw. - was analytisch äquivalent ist - die sätze bestehender steuern erhöht werden.

Aus systematischen Gründen ist es ratsam, zunächst zwischen Steuern auf Bestände und Steuern auf Ströme zu unterscheiden.

Unter den Bestandsteuern verdienen die Grundsteuer. und die Vermögensteuer unsere besondere Aufmerksamkeit.

Grundsteuer muß grundsätzlich jeder bezahlen, der über Grundbesitz verfügt. Hierbei gliedert das Bewertungsgesetz (BewG) den Grundbesitz in

- Betriebe der Land- und Forstwirtschaft,

- Betriebsgrundstücke und

- Grundstücke als Bestandteile des Grundvermögens.

Für unsere Analyse interessant sind lediglich Grundstücke der zweiten und dritten Kategorie. Es würde den Rahmen dieser Arbeit sprengen, wollte man auch noch der steuerlichen Behandlung des land- und forstwirtschaftlichen sektors Rechnung tragen. Die Sonderregelungen, die wir hierbei zu Tage fördern würden, ähneln in ihrer Vielfalt und Konzeptionslosigkeit sehr stark den für den Wohnungssektor charakteristischen Bestimmungen und sollten deshalb Gegenstand einer eigenständigen Arbeit sein.

Sowohl der Wohnungsinvestor als auch der Bodenmakler werden zur Zahlung von Grundsteuer herangezogen. Steuerbemessungsgrundlage ist bei den Wohnungseigentümern der gemeine Wert der bebauten Grundstükke $\left(\mathrm{P}_{\mathrm{H}} \mathrm{H}\right)$, beim Bodenmakler der gemeine Wert des unbebauten Grundstückbestandes $\left(P_{B} B\right)$. Mit $\tau_{g r}$ als Grundsteuersatz betrüge das Aufkommen $T_{g r}$ der Grundsteuer bei einer korrekten Bewertung der Grundstücke

(III.1)

$$
T_{g r}=\tau_{g r}\left(P_{H} H+P_{B} B\right)
$$

D-Mark . 
Es wird aufschlußreich sein, zu untersuchen, - wie eine Grundsteuer wirkt, die dem Ideal aus (III.1) entspricht und

- welche allokativen Konsequenzen sich auf der anderen Seite aus der in Literatur und Praxis so oft bemängelten fehlenden Zeitnähe der Bewertung von Grundstücken ergeben.

In diesem Zusammenhang wird es auch interessant seir, der Frage nachzugehen, inwieweit RICARDOs These von der Neutralität der Grundsteuer in Gestalt einer reinen Bodenwertsteuer berechtigt ist.

Neben der Grundsteuer unterliegt das Grundvermögen zusätzlich noch der Vermögensbesteuerung. Anknüpfungspunkt ist hierbei der Wert des Reinvermögens, also der Wert der (bebauten oder unbebauten) Grundstücke abzüglich des darin gebundenen Fremdkapitals. Da sich der Bodenmakler annahmegemäß nicht verschuldet, fließen dem öffentlichen Sektor bei einer korrekten, zeitnahen Bewertung des Grundvermögens durch die Erhebung einer Vermögensteuer Einnahmen in Höhe von

(III.2)

$$
T_{V}=\tau_{V}\left(P_{H} H-D+P_{B} B\right)
$$

D-Mark zu; $\tau_{v}$ steht in (III.2) für den Vermögensteuersatz. Auch im Rahmen der Vermögensbesteuerung steht zu vermuten, daß es infolge von Bewertungsungenauigkeiten $z u$ (zusätzlichen?) allokativen Verzerrungen kommt.

Als weitere den Wohnungs- und den Baulandsektor belastende Substanzsteuer käme grundsätzlich noch die Gewerbekapitalsteuer in Betracht. Um aber eine Doppelbelastung des gewerblichen Grundbesitzes mit Grundsteuer und Gewerbekapitalsteuer zu vermeiden, hat der Gesetzgeber in $\$ 12$, Absatz 3, Ziffer 1 Gewerbesteuergesetz (GewStG) festgelegt, daß alle im Einheitswert des gewerblichen Betriebes enthaltenen Einheitswerte von Betriebsgrundstucken bei der Ermittlung des steuerpflichtigen Gewerbekapitals abzuziehen sind. Für unsere Modellwelt bedeutet dies, daß die beiden repräsentativen Akteure - soweit die Finanzbehörden deren Tätigkeit ubberhaupt als "gewerblich" interpretieren - nicht der Gewerbekapitalbesteuerung unterliegen. 
Unter dem Stichwort "Besteuerung vor Stromgrößen" wăre zunächst jene Verkehrsteuer zu nennen, der im Zusammenhang mit der Ubertragung von Grundeigentum die gewichtigste Rolle zukommt - die Grunderwerbsteuer nämlich ${ }^{31)}$. Grunderwerbsteuer fällt grundsätzlich bei jeder entgeltlichen Grundstückstransaktion an. Als für unsere zwecke hilfreich erweist sich die Tatsache, daß der Grunderwerbsteuersatz $\tau_{\text {gre }}$ nach neuestem Recht auf einheitlich 2 Prozent des Verkaufswertes festgesetzt und nahezu alle Ausnahmeregelungen des alten Rechts auBer Kraft gesetzt wurden. Damit verhilft die Besteuerung von Baulandtransaktionen dem staat in jeder Periode zu Einnahmen in Höhe von

$$
T_{\text {gre }}=\tau_{\text {gre }} P_{B} F \quad \text { D-Mark. }
$$

Der Grunderwerbsteuer wird häufig nachgesagt, daB sie den Bodenverkäufer veranlaßt, den optimalen Verkaufszeitpunkt (und damit auch den optimalen zeitpunkt der Bebauung) hinauszuzögern. Unsere Analyse wird zeigen, ob diese Kritik berechtigt ist.

DaB ausgerechnet die Umsatzsteuer bei einer Analyse der Besteuerung des Wohnungssektors Berücksichtigung finden soll, mag überraschen, sind doch Umsätze, die aus der Vermietung von Wohnungseigentum fließen, in aller Regel umsatzsteuerfrei ( $\$ 4, Z$ iffer $12 \mathrm{a}$ UStG). Und selbst die Ausnahme von dieser Regel, die Option nach $\$ 9$ UStG, wird es für nach dem 1.1.1985 fertiggestelltes Wohnungseigentum nicht mehr geben, falls die Vermietung Wohnzwecken dient.

31) Weitere für den Grundstlicksverkehr relevante Steuerarten sind die Erbschaftsteuer und Schenkungsteuer. Obwohl auch diese Formen der Besteuerung ökonamische Probleme in sich bergen (die im wesentlichen auf die Unterbewertung der Grundstücke zurückzuführen sind), können sie in dem hier gewählten Modellrahmen vernachlässigt werden, da sie auf die Wahl des Bebauungszeitpunktes wohl keinen Einfluß nehmen. 
Aber gerade die generelle Umsatzsteuerbefreiung der Mieteinnahmen ist es, die die Berücksichtigung dieser steuer so interessant macht. Die steuerbefreiung stellt nämlich eine Sonderbehandlung dar, die der Wohnungsinvestor mit nur wenigen anderen Bereichen unserer Volkswirtschaft teilt. So unterliegen in unserem Modell die Konsumgüterkäufe $C$ nach wie vor der Mehrwertsteuer. $\mathrm{Zu}$ welchen allokativen Effekten diese Steuerbefreiung führen kann, ist eine weitere Frage, um deren Beantwortung wir uns in der folgenden Analyse bemühen.

Bei der Betrachtung von Steuern auf stromgrößen darf natürlich die vom Aufkommen her für den Fiskus bedeutendste Steuer nicht vergessen werden: wie in allen westlichen Industrieländern wird auch in der Bundesrepublik Deutschland das Volkseinkommen der Einkommensteuer unterworfen.

Dem Konzept der volkswirtschaftlichen Gesamtrechnung folgend, läßt sich das Volkseinkommen in seine einzelnen Komponenten - Löhne und Gehälter (wL),

- Zinseinkünfte (rD) und

- Gewinne (G)

zerlegen, wobei es zweckmäßig ist, zusätzlich zwischen entnommenen (bzw. ausgeschütteten) Gewinnen (A) und einbehaltenen (thesaurierten) Gewinnen zu unterscheiden. Jede dieser Einkommenskategorien wird mit einem spezifischen steuersatz belegt.

In welchem Verhältnis der Lohnsteuersatz $\left(\tau_{1}\right)$, der Steuersatz auf Erträge aus Wertpapiervermögen $\left(\tau_{z}\right)$, der steuersatz auf ausgeschüttete $\left(\tau_{a}\right)$ und jener auf einbehaltene Gewinne $\left(\tau_{e}\right)$ zueinander stehen, hängt zum einen vom praktizierten System der Einkommensbesteuerung, zum anderen von der Rechtsform des betrachteten Unternehmens ab. Wir wollen uns im folgenden die Analyse insoweit etwas erleichtern, als wir uns auf die Betrachtung von wohnungsunternehmen beschränken wollen, bei denen es sich um Personengesellschaften handelt. In diesem Falle nämlich wird das Verhältnis der verschiedenen Einkommensteuer- 
sätze zueinander durch den $\S 1$ des Einkommensteuergesetzes (EStG) festgelegt. Ihm zufolge wird die Summe aus Löhnen und Gehältern, Zinseinkünften (als Einkünfte aus Kapitalvermögen) sowie im Rahmen der Vermietung von Wohnungseigentum erzielte (ausgeschütete oder einbehaltene) Gewinne (entweder als Einkünfte aus Vermietung und Verpachtung oder als Einkünfte aus Gewerbebetrieb oder als Einkünfte aus Land- und Forstwirtschaft oder als Einkuinfte aus selbständiger Arbeit) mit einem einheitlichen Steuersatz belegt; es gilt demnach die Beziehung

$$
\left.\tau_{1}=\tau_{z}=\tau_{a}=\tau_{e} \cdot 32\right)
$$

Wie aus (III.4) hervorgeht, gelangt in unserem Modell bei der Bestimmung der Einkommensteuerzahllast im Rahmen unserer Analyse ein proportionaler Tarif zur Anwendung. Damit soll keineswegs die Tatsache geleugnet werden, daß die progression unseres Einkommensteuertarifs schon für sich genommen wohlfahrtsmindernde Konsequenzen zeitigt.

32) Handelt es sich bei dem Wohnungsunternehmen dagegen um eine juristische Person, werden die einbehaltenen Gewinne grundsätzlich anders behandelt als die ausgeschütteten: seit 1976 wird bei uns das sogenannte "Vollanrechnungsverfahren" praktiziert, bei dem zunächst die gesamten Gewinne mit dem Spitzensteuersatz der Einkommensteuertabelle belastet werden; die auf ausgeschüttete Gewinne entfallenen Steuerbeträge werden dem Aktionärshaushalt jedoch erstattet bzw. vergütet, so daß Dividenden letztendlich nur von der persönlichen Einkamensteuer des Anteileigners erfaßt werden. Da der durchschnittliche Einkammensteuersatz des repräsentativen Haushalts aufgrund der Progression des Einkamensteuertarifs geringer ist als der Spitzensteuersatz, gilt in diesem Fall $\tau_{e}>\tau_{1}=\tau_{z}=\tau_{a}$. Wie sich zeigen läßt, haben diese Spezifika keine Auswirkungen auf das Investitionsverhalten des betrachteten Unternehmens - denkbare Effekte werden vielmehr durch den Wechsel in der optimalen Finanzierungsform kampensiert. Aus diesem Grund ist der Ausschluß von Kapitalgesellschaften aus unserer Analyse auch keine so erheblich einengende Maßnahme, wie dies auf den ersten Blick den Anschein haben mag; - vgl. SINN (1982). Zur Problematik der rechtsformabhängigen Besteuerung vgl. SCHREIBER (1984). 
Wir wollen im folgenden lediglich Probleme analysieren, die aus der Abgrenzung der verschiedenen Einkommensteuerbemessungsgrundlagen herrühren. Diese Probleme stehen mit verteilungspolitischen Zielsetzungen in keinem unmittelbaren zusammenhang. Die Einfuhrung eines proportionalen, auf alle Einkunftsarten anzuwendenden Einkommensteuertarifs ist dieser Aufgabenstellung deshalb sicherlich angemessen.

Gleichheit der Steuersätze für die verschiedenen Einkunftskunftsarten eines einzelnen steuerpflichtigen muß nicht notwendigerweise auch bedeuten, daß die Bemessungsgrundlagen, auf die der einheitliche Steuersatz angewandt wird, für verschiedene steuerpflichtige ebenfalls nach einheitlichen Kriterien abgegrenzt werden. Tatsächlich resultiert eine Vielzahl von Problemen, die mit der Besteuerung der Mieteinkünfte einhergehen, eben aus der Tatsache, daß es eine solch einheitliche Abgrenzung der Einkommensteuerbemessungsgrundlage nicht gibt.

Diese Aussage gilt insbesondere für die einkommensteuerliche Erfassung von Wertsteigerungen des Grundstücksvermögens. Solche Wertzuwächse können vielerlei Ursachen haben - sie können zurückgeführt werden

- auf den InflationsprozeB,

- auf infrastrukturelle Maßnahmen der öffentlichen Hand,

- auf die sich im Zeitablauf verändernden Knappheits- und Preisrelationen oder

- auf Manipulationen des Eigners am Grundstück. Besonders die beiden erstgenannten Ursachen nahmen in der finanzpolitischen Diskussion der späten sechziger und frühen siebziger Jahre eine dominierende Stellung ein. Vor allem die Absicht, zumindest einen Teil der sich als Folge von raumplanerischen Maßnahmen sowie der Erschließung von Neubauland einstellenden Wertsteigerungen abzuschöpfen, schlug sich in einer ganzen Reihe ernsthafter Gesetzesvorschläge nieder (so bei- 
spielweise der Vorschlag der SPD zur Einführung eines Planungswertausgleichs oder der der FDP zur Implementierung einer Infrastrukturkostenabgabe). Die Besteuerung inflationsbedingter oder auf Kosten der Allgemeinheit zustandegekommener Wertsteigerungen wird uns im Rahmen dieser Arbeit jedoch nicht beschäftigen; wir wollen uns auf die Besteuerung von Wertzuwächsen konzentrieren, die auf die sich im Zuge des volkswirtschaftlichen Wachstumsprozesses verändernden Knappheitsrelationen zurückgeführt werden können bzw. auf werterhaltende Arbeiten, die die Grundstückseigentümer am Gebäude vornehmen (lassen); insbesondere soll der Frage nachgegangen werden, ob und, wenn ja, in welcher Weise die aktuelle Form der Erfassung und Besteuerung von Wertzuwächsen den gesamtwirtschaftlichen Allokationsproze $\beta$ verzerrt und wie und wann Wertzuwächse sinnvollerweise erfaßt werden sollen, um solche Verzerrungen zu vermeiden.

Schlägt die Nutzung von Wohnungseigentum nach Auffassung der Finanzverwaltung in eine gewerbliche Tätigkeit um, muß Derjenige, der die Nutzung betreibt, zusätzlich zur Last der Einkommensteuer - mit Einschränkungen - die Last der Gewerbeentragsteuen tragen.

Die Einschränkungen beziehen sich auf den $\S 9$, Ziffer 1 , Sätze 1 bis 4 GewStG. Denn zur Vermeidung bzw. Minderung der (vermeintlichen) Doppelbelastung ${ }^{33)}$ gewerblicher Mieterträge mit zwei Objektsteuern sieht $\$ 9$, ziffer 1 , Satz 1 GewStG vor, daß 1,2 Prozent des gemäß §121a BewG um 40 Prozent erhöhten Einheitswertes der Betriebsgrundstücke vom steuerpflichtigen Gewerbeertrag des Wohnungsunternehmers abzugsfähig sind - sofern nicht auf Antrag die erweiterte Kürzung des §9, Ziffer 1, Sätze 2 bis 4 GewStG zulässig ist, nach der sämtliche bei der Ermittlung des Gewerbeertrags im Gewinn enthaltenen Erträge aus der Nutzung des eigenen Grundbesitzes zur Kürzung herangezogen werden können.

33) Der in den Gesetzeskommentaren verwendete Begriff der Doppelbelastung kann leicht zu Mißverständnissen führen, da die Gewerbeertragsteuer an einer Stramgröße, die Grundsteuer dagegen an einer Bestandsgröße anknüpft. Mit dem gleichen Recht könnte man auch von einer Doppelbelastung der Anlagegüter durch Einkammensteuer und Vermögensteuer sprechen. 
Führt die Normalkürzung des $§ 9$, Ziffer 1, Satz 1 GewStG aufgrund des zu gering angesetzten Pauschalbetrags lediglich zu einer Minderung der Gewerbeertragsteuerbelastung, so hat die Anwendung des $\S 9$, Ziffer 1, Sätze 2 bis 4 GewStG die völlige Vermeidung dieser zusätzlichen Steuerlast zur Folge. Hätten alle gewerblichen Wohnungsunternehmer die Möglichkeit, für die erweiterten Kürzungen $z u$ optieren, würde sich demzufolge auch eine explizite Berücksichtigung der Gewerbeertragsteuer in unserer Analyse erübrigen. Bemerkenswerterweise aber hat der Gesetzgeber die Bewilligung des Antrags auf erweiterte Kürzungen an spezielle, ökonomisch schwer begründbare Voraussetzungen geknüpft.

So kann den $\S 9$, Ziffer 1, Sätze 2 bis 4 GewStG nur derjenige in Anspruch nehmen, welcher neben der Funktion des Wohnungsunternehmers keine andere unternehmerische Funktion ausübt. Darüberhinaus darf es sich bei der Verwaltung und Nutzung eigenen Grundbesitzes nicht um eine gewerbliche Tätigkeit handeln - dies fordert $\S 62$, Absatz 1, Ziffer 1 Gewerbesteuerrichtlinien (GewStR); den gewerblichen Status trägt der betrachtete Wohnungsunternehmer dann nicht qua Betätigung, sondern qua Rechtsform seines Unternehmens. 34 )

Wohnungsinvestoren, die neben der Verwaltung eigenen Grundbesitzes noch andere unternehmerische Tätigkeiten ausüben ${ }^{35}$ ) können sich dagegen nur auf die "doppelbelastungs"-mindernden Vergünstigungen des $\S 9$, Ziffer 1 , Satz 1 GewStG berufen. Im Interesse der Konsumenten von Wohnleistungen wollen wir uns bemühen, zu einem normativen Urteil über diese Art der Besteuerung zu kommen.

34 ) Zur Unterscheidung zwischen gewerblicher und vermögenswaltender Tätigkeit vgl. Kapitel III, Abschnitt 5.2.2.2.6.

35) Welche Tätigkeiten hierbei in Frage kommen, hat der Bundesfinanzhof in einer Reihe von Urteilen zu klären versucht;- vgl. auch GewStR, $\S 62$, Absatz 1, Ziffer 2. 
Grundsteuer, Vermögensteuer, Grunderwerbsteuer, Umsatzsteuer Einkommensteuer und Gewerbeertragsteuer sind diejenigen steuern, auf deren Betrachtung wir uns im folgenden konzentrieren wollen.

Wie einçangs schon erwähnt, sind für etwaige, mit diesen Besteuerungsformen verknüpfte Wohlfahrtsverluste ausschließlich die durch die steuererhebung ausgelösten Substitutionseffekte ursächlich. Diese lassen sich analytisch isolierer, iridem man den mit der Besteuerung ebenfalls einhergehenden Einkommenseffekt durch die Annahme neutralisiert, daß der öffentliche Sektor seine gesamten Steuereinnahmen zur Finanzierung eines Transferprogramms verwendet, durch dessen Vollzug jeder einzelne private Haushalt genau jenen Betrag zurückerhält, den er im Zuge der Besteuerung an den öffentlichen sektor abführen mußte.

Bei der Interpretation der von der Modellalgebra zu liefernden Analyseergebnisse sind uns infolge der partialanalytischen Betrachtungsweise des Kapitels II gewisse Grenzen gesetzt: so sind Aussagen über steuerinduzierte intersektorake Verzerrungen zwar bezüglich der Wohnungs-, Bauland- und Kapitalmarktsektoren, nicht aber zwischen jedem dieser drei sektoren einerseits und den restlichen Sektoren einer Volkswirtschaft andererseits möglich. Denn auch für diese restlichen Sektoren kennen die Steuergesetze eine ganze Reihe von Sonderregelungen, die sich einer pauschalen Wertung entziehen. Auch können wir steuerinduzierten Verzerrungen, die außerhalb der Unternehmenssektoren entstehen, nicht ohne weiteres Rechnung tragen: als bekanntestes Beispiel hierfür wäre der Einfluß der Einkommensbesteuerung auf die Arbeitszeit-Freizeit-Entscheidung der privaten Haushalte zu nennen.

In Anbetracht dieser Verzerrungen, die sich im Rahmen unseres Partialmodells nicht fassen lassen, wollen wir unseren normativen Maßstab etwas tiefer ansetzen: Allokationseffizient im Sinne des second best-postulats ist nur eine solche Form der Besteuerung des Wohnungs - und Baulandsektors, die über modellexterne wohlfahrtsverluste hinausgehende Ineffizienzen vermeiden hilft. 
2. Die Besteuerung des Immobilienvermögens

2.1. Die Grundsteuer

2.1.1. Die Grundsteuer geltenden Rechts

2.1.1.1. Einige instititionelle Details

Um die Allokationswirkungen dieser Steuer würdigen zu können, ist es erforderlich, einen Blick auf die institutionellen Regelungen und Eigentümlichkeiten zu werfen, gemäß denen die Grundsteuer in der Bundesrepublik Deutschland festgesetzt und erhoben wird.

Steuersystematisch zählt die Grundsteuer zu den objekt(Sach-, Real-)Steuern. Gegenstand der Besteuerung ist eine Sache, nämlich der Grundbesitz im Sinne des Bewertungsrechts; die persönlichen Verhältnisse des Grundstückseigentümers werden bei der Festsetzung der Grundsteuerzahllast nicht berücksichtigt. Anknüpfungspunkt der steuer ist der Wert des Grundbesitzes, der wiederum nach den Vorschriften des Bewertungsgesetzes berrechnet wird. Letzteres bestimmt, daß als Bemessungsgrundlage der "gemeine Wert" ( $\$ 9$ BewG) des Grundstücks zu dienen habe, der Wert also, der im gewöhnlichen Geschäftsverkehr bei einer VeräuBerung am Bewertungsstichtag zu erzielen wäre. Dabei unterscheidet das Gesetz - ganz im Sinne unserer Analyse zwischen baureifen und bebauten Grundstücken.

Zu den baureifen Grundstücken, wie sie durch $\$ 73$ Absatz 1 BewG bezeichnet werden, zählen alle Flächen, sofern sie

- in einem rechtsverbindlichen Bebauungsplan ( $§ \S 8 \mathrm{ff}$. Bundesbaugesetz (BBauG)) als Bauland ausgewiesen sind,

- ihre sofortige Bebauung rechtlich und tatsächlich möglich ist und

- die Bebauung innerhalb des Plangebiets in einem benachbarten Bereich begonnen hat oder schon durchgeführt ist.

Demnach handelt es sich bei dem in unserer Analyse betrachteten Baulandbestand B um baureifes Land im Sinne des Bewertungsgesetzes. Bewertungsmaßstab dieses Bestandes ist - den Bestimmungen des $\S 9$ Absatz 1 BewG i.V.m. $\$ 17$ Absatz 3 BewG folgend - grundsätzlich der aktuelle Bodenpreis $\mathrm{P}_{\mathrm{B}}$. In der Praxis 
läßt sich $\mathrm{P}_{\mathrm{B}}$ durch einen Kaufpreisvergleich oder, wo dies nicht möglich ist, durch die Verwendung von Richtwerten ermitteln.

Bei den bebauten Grundstücken unterscheidet das Bewertungsgesetz zwischen sechs Arten, von denen vier für unsere wohnungsmarktwirtschaftliche Analyse von Interesse sind:

- Mietwohngrundstücke, die mehr als zwei Wohnungen aufweisen und zu mehr als 80 Prozent zu Wohnzwecken genutzt sind,

- gemischtgenutzte Grundstücke, die z.T. jedenfalls auch für Wohnzwecke nutzbar sind, sowie

- Einfamilienhäuser und

- Zweifamilienhäuser.

Entsprechend der Bewertung baureifer Grundstücke müßte bei der Bewertung bebauter Grundstücke der Häuserpreis $P_{H}$ zugrundegelegt werden, hinter dem sich der Barwert der Nettomieterträge, bezogen auf eine Wohnflächeneinheit, und folglich auch die potentielle Zahlungsbereitschaft eines potentiellen Grundstückskäufers für diese Wohnflächeneinheit verbirgt. Die Erfahrung zeigt jedoch, daß hierbei nur schwer überwindbare Probleme auftreten - vor allem deshalb, weil der tatsächliche Häuserpreis $\mathrm{P}_{\mathrm{H}}$ in der Praxis nur in Ausnahmefällen durch einen Vergleich der Verkaufspreise zu ermitteln sein wird. Diese Schwierigkeiten umgeht die Finanzverwaltung dadurch, daß sie den gemeinen Wert bebauter Grundstücke im allgemeinen mit Hilfe eines pauschalierten Ertragswertverfahrens $z u$ schätzen versucht ${ }^{36)}$. Hierbei sollte, um möglichen Mißverständnissen vorzubeugen, betont werden, daß der (geschätzte) Wert bebauter Grundstücke nicht den Wert nur des Bodens widerspiegeln soll; der Grundstückswert, wie ihn der Gesetzgeber versteht, umfaßt sowohl den reinen Bodenwert als auch den Wert des aufstehenden Gebäudes zuzüglich des Wertes der AuBenanlagen.

36) Das sogenannte "Sachwertverfahren" findet nur in Ausnahmefällen Anwendung, beispielsweise bei der Bewertung von Einfamilien- und Zweifamilienhäusern, wenn sich diese durch besondere Gestaltung und Ausstattung von den im Ertragswertverfahren bewerteten Ein- und Zweifamilienhäusern unterscheiden;- vgl. SCHÖNHOFER/REINISCH, Gruppe 15. 
Geschätzt wird der Grundstückswert durch die Anwendung eines Vervielfäligers auf die Jahresrohmiete. Die Jahresrohmiete ist das Gesamtentgelt, das der Mieter (oder Pächter) für die Benutzung des Grundstücks aufgrund vertraglicher Vereinbarungen nach dem Stand im Feststellungszeitpunkt des Einheitswertes $z u$ entrichten hat. 37 , 38)

Im Vervielfältiger berücksichtigt sind die Bewirtschaftungskosten (Verwaltungskosten, Instandhaltungskosten, Mietausfallwagnis und Betriebskosten), ebenso die Bauart des Gebäudes (welche Rückschlüsse auf dessen Lebensdauer gestattet) und die Größe der Gemeinde, innerhalb deren Gemarkung das Gebäude errichtet wurde - dies deshalb, weil das Mietenniveau erfahrungsgemäß mit der Bevölkerungsdichte steigt. Darüberhinaus nehmen die Multiplikatoren auch in Abhängigkeit von der Grundstücksart unterschiedliche werte an.

Sieht man von diesem letztgenannten Phänomen einmal ab das in aller Regel dazu führt, daß der Einheitswert eines im Ertragswertverfahren geschätzten Einfamilienhauses (oftmals beträchtlich) iuber dem eines vergleichbaren Zweifamilienhauses liegt so läßt sich angesichts der konzeptionellen Ausgestaltung des zur Schätzung des gemeinen wertes verwendeten Pauschalierungsverfahrens wohl zurecht das Urteil vertreten, daß es sich hierbei zumindest von der Idee her um eine schematisierte Reiner-

37) Es ist die Sollmiete anzusetzen; etwaige Mietausfälle bleiben unberücksichtigt.

38) Bei Grundstücken, für die am Bewertungsstichtag Grundsteuervergünstigungen nach den in \$79BewG bezeichneten Wohnungsbaugesetzen gewährt wurde, ist die Jahresrohmiete um 12 Prozent zu erhöhen, da die normale Grundsteuerbelastung im Vervielfältiger bereits berücksichtigt ist. 
tragskapitalisierungsmethode handelt ${ }^{39}$; seine Anwendung ist aus Gründen der verwaltungstechnischen Vereinfachung und Kosteneinsparung auch aus allokationstheoretischer sicht $\mathrm{zu}$ rechtfertigen - jedenfalls solange keine begründeten $\mathrm{Zweifel}$ dahingehend bestehen, daß der geschätzte Einheitswert weitestgehend mit dem tatsächlichen Marktwert (Verkehrswert) des Grundstücks übereinstimmt. In diesem Fall erhalten wir als Bemessungsgrundlage der von dem Wohnungseigentümer $\mathrm{zu}$ zahlenden Grundsteuer den Ertragswert

$$
E W_{W}=P_{H} H \text {. }
$$

Die Bemessungsgrundlage bei der Besteuerung baureifer Grundstücke lautet analog

$$
\text { (III.6) } \quad \mathrm{EW}_{\mathrm{b}}=\mathrm{P}_{\mathrm{B}} \mathrm{B} \text {. }
$$

Zweifel, daß die derzeit gültigen Einheitswerte mit den aktuellen Marktwerten übereinstimmen, sind allerdings durch den Umstand begründet, daß die Finanzverwaltung auf eine zeitnahe Bewertung bzw. auf eine entsprechende Wertfortschreibung bei Grundstücken verzichtet. Tatsächlich ist es den Finanzämtern bis zum heutigen Tag nicht einmal gelungen, den durch das Bewertungsgesetz ( $\$ 21$ Absatz 1 , Ziffer 1 BewG) verbindlich vorgeschriebenen zeitraum von sechs Jahren einzuhalten, innerhalb dessen eine neue Hauptfeststellung der Einheitswerte erfolgen sollte; die letzte Hauptfeststellung fand schon zum 1. 1. 1964 mit Wirkung zum 1. 1. 1974 statt. Angesichts der in den letzten zwei Dekaden beobachtbaren Bodenwertsteigerungen und der Tatsache, daß der ohnehin zu gering bemessene vierzigprozentige Wertzuschlag auf die zum 1.1.1964 gültigen Einheitswerte für die Zwecke der Grundbesteuerung irrelevant ist, melden sich Bedenken hinsichtlich der Zeit-

39) ROSE (1982), S. 
nähe der praktizierten Bewertungsverfahren an. Diese werden auch dann nicht geringer, wenn man weiß, daß die 1964er Einheitswerte zwar das zweieinhalb- bis dreifache der im Jahre 1935 festgestellten Einheitswerte betragen, die Einheitswerte bebauter Grundstücke aber schon 1935 um bis zu neunzig Prozent unter den entsprechenden Verkehrswerten lagen. Aus diesem Grunde vermuten FRIAUF/RISSE/WINTERS, daß das Niveau der Einheitswerte schon 1964 um etwa ein Drittel unter dem der entsprechenden Verkehrswerte lag. Lediglich die Einheitswerte unbebauter Grundstücke hätten in etwa den Verkehrswerten von 1964 entsprochen. ${ }^{40)}, 41$ )

Wir wollen dieser institutionellen Besonderheit in unserer Analyse durch eine modifizierte Formulierung der Grundsteuerbemessungsgrundlage Rechnung tragen:

$$
E W_{W}^{*}=\gamma_{1} P_{H} H^{\prime}, O \leq \gamma_{1} \leq 1 .
$$

Der Faktor $\gamma_{1}$ kann werte zwischen Null und Eins annehmen, wobei die Grenzwerte die Situation einer völligen Grundsteuerbefreiung $\left(\gamma_{1}=0\right) \mathrm{bzw}$. einer korrekten und zeitnahen Bewertung $\left(\gamma_{1}=1\right)$ des Grundbesitzes kennzeichnen.

Für die Zwecke unserer Analyse wollen wir darüberhinaus unterstellen, daß die Bewertung baureifer Grundstücke den tatsächlichen Verkehrswert wiedergibt. Diese Annahme entspricht zwar nicht ganz den tatsächlichen Gegebenheiten, andererseits

40) FRIAUF/RISSE/WINTERS(1978), S.21.

41) Um das Ausmaß der Unterbewertung zu begrenzen, schreibt Artikel 7 des Steueränderungsgesetzes vam 19.8.1969 (Bundesgesetzblatt I(1969), S.1211) vor, daß jede Bewertung mindestens den Betrag ergeben muß, der 50 Prozent des Wertes beträgt, mit dem der Grund und Boden allein, ohne Außenanlagen, anzusetzen wäre. Aus unserer Analyse der Laisser-FaireÖkonomie wissen wir aber, daß diese Begrenzung unterer ökonomischen Gesichtspunkten wenig Sinn macht: wegen $\hat{\mathrm{P}}_{\mathrm{B}}=\hat{\varepsilon}>\hat{\mathrm{P}}_{\mathrm{H}}=\beta \hat{\varepsilon}$ wird der Wert des unbebauten Bodens nach absehbarer Zeit zwangsläufig jenen gleichgroßer, aber bebauter Grundstiucke um 100 prozent übertreffen. 
ist es aber auch eine Tatsache, daß die Eigentümer baureifer Grundstücke durch die geltenden Bewertungsvorschriften weit weniger begünstigt werden als die Eigentümer bebauter Grundstücke.42) Und da für unsere Uberlegungen neben der Existenz einer Grundsteuer lediglich die differentiellen Unterschiede in der grundsteuerIichen Behandlung bebauter und unbebauter Grundstücke von Interesse sind, ist die Annahme einer zeitnahen Bewertung baureifen Grund und Bodens durchaus akzeptabel. Die abzuleitenden Ergebnisse verlieren dadurch für die reale welt nichts an Relevanz.

Bei dex Veranlagung und der Erhebung der Grundsteuer ist nun eine weitere Besonderheit $\mathrm{zu}$ beachten. Diese resultiert aus der Tatsache, daß das Aufkommen der Grundsteuer durch Artikel 106, Absatz 6 Grundgesetz den Gemeinden zugestanden wird; diese besitzen auch weitgehende Freiheiten in der Festsetzung der Grundsteuerlast: Nachdem der Einheitswert des Grundstücks ermittelt ist, wird durch Anwendung eines, bezogen auf eine gegebene Grundstücksart, bundeseinheitlichen Promillesatzes, der sogenannten Steuermeßzahl, auf den Einheitswert der GrundsteuermeBbetrag errechnet.

Dagegen variiert die steuermeBzahl in Abhangigkeit von der Grundstücksart; während sie i.d.R. (also auch für baureifen Boden) bei 3,5 Promille liegt, bilden Ein-und Zweifamilienhäuser insofern eine Ausnahme, als die steuermeBzahl

- bei Zweifamilienhãusern bei 3,1 promille und

- bei Einfamilienhäusern bei 2,6 promille (für die ersten 70000 D-Mark des Einheitswertes) bzw, bei 3,5 promille (für den diese 70000 D-Mark übersteigenden Betrag) liegt.

42) Diese Aussage erhält zusätzliches Gewicht, wenn man sich daran erinnert, daß gleichzeitig mit der 4o-prozentigen Anhebung der Einheitswerte die vorher guiltigen Steuermeßzahlen gesenkt wurden. Iediglich bei unbebauten Grundstïcken, für die der Grundsatz der Steuerneutralität der Grundsteuerreform von 1973 nicht galt, wurde die Steuermeßzahl nicht im Verhältnis zur Erhöhung der Einheitswerte herabgesetzt. Die effektive Grundsteuerbelastung ist samit bei bebauten Grundstiicken konstant geblieben, bei unbebauten Grundstücken dagegen gestiegen. 
Auf der Grundlage des Steuermeßbetrags wird von den hebeberechtigten Gemeinden mit dem Grundsteuerbescheid die zu zahlende Grundsteuer festgesetzt. Dazu bestimmt das Gemeindeparlament für ein oder mehrere Kalenderjahre, mit welchem prozentsatz (Hebesatz ( $\$ 25$ Grundsteuergesetz)) des Steuermeßbetrags die Grundsteuer zu erheben ist.3),44)

Für die Zwecke unserer Analyse wollen wir von den grundstücksartspezifischen Abweichungen in den Steuermeßzahlen abstrahieren und Steuermeßzahl und Hebesatz zu einem Grundsteuersatz $\tau_{\text {gr }}$ zusammenfassen.

2.1.1.2. Die Besteuerung baureifer Grundstücke und die Planungsprobleme van Bodenmaklern und wohnungsinvestoren

Aus den vorangegangenen Ausführungen wissen wir, daß der Bodenmakler in jeder Periode Grundsteuer in Höhe von

(III. 8)

$$
T_{g r}=\tau_{g r} P_{B} B
$$

D-Mark zahlen muß. Um zu sehen, ob und wie die Besteuerung des Grund und Bodens auf seine Verkaufspläne Einfluß nimmt, müssen wir diese Substanzsteuer in das Laisser-Faire-Entscheidungsproblem (II.46) einbauen. Ziel des Bodenmaklers muß es unter diesen geänderten Bedingungen nun sein, durch eine optimale Wahl des Zeitpfades der Bodenverkäufe den Barwert der Bodenverkaufserlöse abzüglich der zu zahlenden Grundsteuer zu maximieren.

43) Die Hebesätze sind getrennt festzulegen; und zwar einheitlich

- für alle Betriebe der Land- und Forstwirtschaft in der Gemeinde (Grundsteuer A) und

- für alle Grundstücke ind der Gemeinde (Grundsteuer B) .

44) Die Hebesätze streuen in der Bundesrepublik derzeit zwischen 100 Prozent und über 500 Prozent. Im Mittel liegen sie etwa bei 260 Prozent;vgl. ROSE' (1982). 
Formal stellt sich dieses Optimierungsproblem wie folgt dar:

(III.9)

$$
\left\{F^{a}\right\} \quad M_{B}\left(t_{0}\right) \equiv \int_{t_{0}}^{\infty}\left[P_{B}(t) F^{a}(t)-z \tau_{g r} P_{B}(t) B(t)\right] \exp \left[-\operatorname{zr}\left(t-t^{*}\right)\right] d t,
$$

unter den Bedingungen

(II.1)

$$
B\left(t_{0}\right)>0 \text {, }
$$

(II. 17a)

$$
z= \begin{cases}0 & \text { für } t o t<t * \\ 1 \text { für } \quad t \geq t *\end{cases}
$$

$$
\dot{B}=-F^{a} \text {. }
$$

Die in laufenden Werten formulierte Hamiltonfunktion lautet deshalb

(III.10)

$$
H=P_{B} F^{a}-\tau_{g r} P_{B} B-\lambda_{B} F^{a},
$$

wobei $\lambda_{B}$ wiederum für den Schattenpreis des Bodenbestandes $B$ steht. Differenziert man (III.10) nach der Kontrollvariablen $F^{a}$ und nach der Zustandsvariablen $B$, so errechnet man als Bedingungen für den gleichgewichtigen Zeitpfad des Bodenangebots

$$
\begin{aligned}
& \text { (III.11a) } \quad \hat{\mathrm{P}}_{\mathrm{B}}=0 \text { für } t_{0} \leq t<t^{*} \text {, } \\
& \text { (III.11b) } \quad \hat{\mathrm{P}}_{B}=r+\tau_{g r} \quad \text { für } \quad t \geq t * \text {. }
\end{aligned}
$$

Vergleicht man die Laisser-Faire-Bedingungen (II.50a) und (II.5Ob) mit den oben abgeleiteten Gleichgewichtsbedingungen, so erkennt man, daß die Einführung einer Grundsteuer geltenden Rechts auf die Verkaufspläne des repräsentativen Bodenmaklers EinfluB nimmt. Denn es bietet sich ihm die Möglichkeit, durch eine Revision seines ursprünglichen Baulandangebots der Grundsteuerlast zumindest partiell auszuweichen. Diese Möglichkeit besteht darin, daß er in unmittexbarem Anschluß an die erstmalige Erhebung der steuer sein Baulandangebot erhöht und den dabei realisierten Verkaufserlös für den Kauf grundsteuer- 
Greier Aktiva verwendet:

- infolge des in $t_{0}$ erhöhten Bodenangebots wird c.p. der Bodenpreis $\mathrm{P}_{\mathrm{B}}\left(t_{0}\right)$ und mit ihm die Grundsteuerbelastung dieser Periode sinken;

- als Konsequenz dieser geänderten Angebotsstrategie kommt es darúberhinaus - verglichen mit der Laisser-FaireSituation - zu einer Verknappung des Bodenangebots zukünftiger Perioden. Setzt man die Gleichgewichtsbedingung (III.1 $1 \mathrm{~b}$ ) in die Bestimmungsgleïchung für die Veränderungsrate des Bodenverbrauchs

$$
\hat{F}=\hat{a}-\hat{P}_{B}(n \beta+\alpha)=\text { konstant }<0
$$

ein, so erkennt man, daß der Bodenverbrauch im neuen Gleichgewicht unter dem EinfluB der Grundsteuer von Periode zu Periode stärker abnimmt, als dies noch in der steuerfreien Welt der Fall gewesen war; dementsprechend wird auch das Baulandangebot selbst, trotz der anfänglichen Mobilisierung, in absehbarer zeit unter seinem Laisser-Faire-Niveau liegen; bei gegebener Entwicklung der Baulandnachfrage mu dies $\mathrm{zu}$ einer Beschleunigung des Wachstums des gleichgewichtigen Bodenpreises führen.

Der optimale Bodenverkaufsplan ist nach Aussage von (III.19b) der, bei dem der Wertzuwachs baureifer Grundstücke gerade ausreicht, die Opportunitätskosten der Hortung von Bauland in Form von zinskosten und Grundsteuerlasten zu decken; auf diese Weise stimmt die Rendite, die das Horten von Bauland abwirft, nach steuer mit den potentiellen Erträgen alternativer und grundsteuerfreier Anlageformen überein.

Daß der Bodenmakler auch tatsächlich gemäß der Handlungsanweisung der modifizierten HOTELLING-Regel aus (III.11b) vorgehen kann, setzt selbstverständlich voraus, daß der Wohnungsinvestor bereit und in der Lage ist, diese unter den geänderten Bedingungen nun optimale Strategie auch mitzutragen: wenn Bedin- 
gung (III.1.1b) tatsächlich einen 'Teil des neuen Gleichgewichts beschreiben soll, muß die Zahlungsbereitschaft der Wohnungsinvestoren für Bauland mit derselben Rate wachsen wie der opportunitätskostendeckende Angebotspreis $P_{B}$ des Bodens. Wie wir aus der Analyse des Kapitels II wissen, verbirgt sich hinter der zeitlichen Entwicklung dieser Zahlungsbereitschaft die zeitliche Entwicklung des Bodenwertgrenzproduktes und hinter diesem wiederum die Dynamik der Wohnleistungsnachfrage. Nur wenn die Nachfrage nach Wohnraum mit einer Rate wächst, die die Bedingung

$$
\hat{\mathbf{a}}>n \beta \hat{\mathrm{P}}_{\mathrm{B}}
$$

erfüllt, können die Bodenmakler damit rechnen, daß sie ihre revidierten Verkaufspläne am Markt auch durchsetzen können.

Genau diese Bedingung läuft nun aber Gefahr, durch die Erhebung einer Grundsteuer verletzt zu werden. Denn während die Nachfrage nach Wohnleistungen in der Laisser-Faire-రkonomie wegen (II.59) lediglich mit einer Rate

$$
\text { (III.12) } \hat{a}>n \beta r
$$

wachsen mußte, um für den Wohnungsinvestor eine permanente Wohnungsneubautätigkeit rentabel werden $\mathrm{zu}$ lassen, ist im Falle der Erhebung einer Grundsteuer geltenden Rechts hierfür nun die größere Rate

$$
\text { (III.13) } \hat{a}>n \beta\left(r+\tau_{g r}\right)
$$

Voraussetzung: nur dann wird der Wohnungsinvestor bereit sein, die vom Bodenmakler jenseits von $t *$ geforderten Bodenpreise $z u$ zahlen.

Ist Bedingung (III.13) nicht erfüllt, wird der Bodenmakler unter dem Eindruck der von ihm zu tragenden Grundsteuerlast versuchen, im AnschluB an die Einfübrung der steuer seinen gesamten in $t_{0}$ vorhandenen Bestand an baureifen Grundstücken so schnell 
wie möglich zu verkaufen. Denn er weiß, daß die Wohnungsinvestoren in aller zukunft nicht nur nicht bereit sein werden, den von ihm geforderten opportunitätskostendeckenden Bodenpreis zu bezahlen, sondern daß darüberhinaus die Spanne zwischen Angebots- und Nachfragepreis umso gröBer wird, je weiter er den Verkauf hinauszögert.

In unserem Modell würde der gesamte Baulückenbestand $B\left(t_{0}\right)$ deshalb in der Kunstzeitphase $\left(t_{0}, t *\right)$ geschlossen werden; jenseits von t* wäre jede Neubautätigkeit erloschen,

Für uns interessanter ist jedoch der Fall, in dem die wachsbedingung (III.13) erfüllt ist und es folglich auch nach t* zu Bodentransaktionen und Wohnungsneubau kommen wird.

Da in diesem Abschnitt von der Besteuerung bebauter Grundstücke noch abgesehen wird, läßt sich das optimale Verhalten des Wohnungsinvestors in dem hier betrachteten Fall mit Hilfe der folgenden, in Kapitel II abgeleiteten Gleichgewichtsbedingungen beschreiben:

$$
\begin{aligned}
& P_{H} \varphi^{\prime}(\varepsilon)=1, \\
& \frac{\beta}{\alpha} \varepsilon\left\{\begin{array}{l}
< \\
=
\end{array}\right\} P_{B} \Rightarrow F^{d}\left\{\begin{array}{l}
=\} \\
\geq j
\end{array} 0\right.
\end{aligned}
$$

$$
\hat{\mathrm{P}}_{\mathrm{H}}=0
$$

für $t_{0} \leq t<t^{*}$

(III. 16b)

$$
\hat{\mathrm{P}}_{\mathrm{H}}=-\frac{\mathrm{m}}{\overline{\mathrm{P}}_{\mathrm{H}}}+\mathrm{r}+\alpha \delta \text { für } t \geq t^{*}
$$

Angesichts dieser rein optisch unveränderten Entscheidungsregeln nun aber den Schluß zu ziehen, daß der Wohnungsinvestor von der Besteuerung baureifer Grundstücke völlig unbeeindruckt bleibt, wäre falsch; tatsächlich ist das Gegenteil der Fall, und dies in mehrfacher Hinsicht:

- offensichtlich gelingt es dem Bodenmakler nur dann, in der Ausgangsperiode sein zusätzliches Angebot an baureifen Grundstücken am Markt unterzubringen, wenn er bereit ist, dafür 
einen geringeren Bodenpreis $\mathrm{P}_{B}\left(t_{0}\right)$ zu akzeptieren. Sinkende Bodenpreise haben bei konstanten Preisen für Bauleistungen zur Folge, daß der Wohnungsinvestor seine in der steuerfreien Welt hinsichtlich der Kapitalintensität der Bebauung getroffene Entscheidung zugunsten eines vermehrten Einsatzes von Bauland bei der Produktion von Wohnleistungen revidiert (vgl. Bedingung (III.15)). Darüberhinaus haben sinkende Baulandpreise auch ein generelles Absinken der Produktionskosten von Wohnflächeneinheiten zur Folge; dieser Umstand wird den Wohnungsinvestor dazu veranlassen, zumindest in der Ausgangsperiode $t_{0}$ seine Wohnungsneubauinvestitionen im Vergleich zur steuerfreien Welt auszudehnen. In formaler Hinsicht bestätigt dies Bedingung (III.14): die sinkende Kapitalintensität der Bebauung führt zu einem Anstieg des physischen Grenzprodukts des Produktionsfaktors "Bauleistungen"; infolge der Konstanz des Bauleistungspreises kann (III.14) deshalb nur dann erfüllt sein, wenn der Häuserpreis $P_{H}$ und mit ihm das Mietenniveau $m(a / H)$ fällt; bei gegebener Nachfrage nach Wohnleistungen wird dies aber nur dann der Fall sein, wenn der Wohnflächenbestand $\mathrm{H}$ zunimmt.

- dem Wohnungsinvestor ist zudem bekannt, daB es dem Bodenmakler gelingt, durch eine Verknappung des zukünftigen Baulandangebots zumindest einen Teil der Grundsteuer last über steigende Bodenpreise an ihn weiterzugeben. Seine Reaktionen auf diese steuerinduzierte Verhaltensänderung des Bodenmaklers kommen nun nicht alleine dadurch zum Ausdruck, daß in zukunft seine Baulandkäufe in einem stärkeren Maße abnehmen, die Kapitalintensität der Bebauung dagegen schneller zunimmt, als dies noch in der Laisser-Faire-ökonomie geplant war (aus (III.15) folgt in Verbindung mit (III.1†b) $\hat{\varepsilon}=r+\tau_{g r}$ ); dadurch, daß auch die Wachstumsrate des Wohnflächenbestandes unter dem Einfluß der vom Bodenmakler zu zahlenden Grundsteuer abnimmt - dies folgt aus

(II.71) $\quad \hat{\mathrm{H}}=\hat{\mathrm{a}}-\eta \hat{\beta} \hat{\mathrm{P}}_{\mathrm{B}}$ 
in Verbindung mit Bedingung (III.11b) - und so der Wohnflächenbestand $\mathrm{H}$ trotz des anfänglichen Neubauschubs in absehbarer Zeit unterhalb seines Laisser-Faire-Niveaus liegen wird, gelingt es dem Wohnungsinvestor durch die hiermit einhergehende Einschränkung des Wohnleistungsangebots zukünftiger Perioden, die auf ihn überwälzte Grundsteuerlast - zum Teil zumindest an die Mieter selner Wohnungen über steigende Mietpreise weiterzugeben.

Die grundsteuerbedingt beschleunigt wachsenden Wohnungsmieten schlagen sich zudem in einem stärkeren Wachstum der Häuserpreise nieder: da aus (III.14)

$\left(\operatorname{III.17)} \quad \hat{\mathrm{P}}_{\mathrm{H}}=\beta \hat{\varepsilon}\right.$

und aus (III.15)

(III.18) $\quad \hat{\varepsilon}=\hat{\mathrm{P}}_{B}$

folgt, erhält man für die Wachstumsrate des Häuserpreises wegen (III.1 $\mathrm{b}$ )

$$
\hat{\mathrm{P}}_{\mathrm{H}}=\beta\left(r+\tau_{g r}\right)
$$

In Abb. 8 werden die dynamischen Folgen dieser steuerinduzierten Planrevisionen graphisch abgeleitet. Ausgehend von der optimalitätsbedingung (III.11b) kann man zeigen, daß die isoelastischen Entwicklungspfade im rechten Quadranten der Abb.7 als Folge der Änderung im Angebotsverhalten des Bodenmaklers flacher verlaufen müssen. Für die steigung dieser Kurven hatten wir die Beziehung

(II. 77)

$$
\frac{d H}{d B}=\frac{H\left[\hat{a}-n \beta \hat{P}_{B}\right]}{B\left[\hat{a}-(n \beta+\alpha) \hat{P}_{B}\right]}<0
$$

augeleitet. Steigt nun infolge der Grundstücksbesteuerung die Wachstumsrate $\hat{\mathrm{P}}_{\mathrm{B}}$ an, so nimmt der Absolutwert der steigung $\mathrm{dH} / \mathrm{dB}$ offensichtlich $\mathrm{ab}$ - in Abb. 8 dreht sich der Laisser-Faire-Ent- 
$\mathrm{Abb} .8$

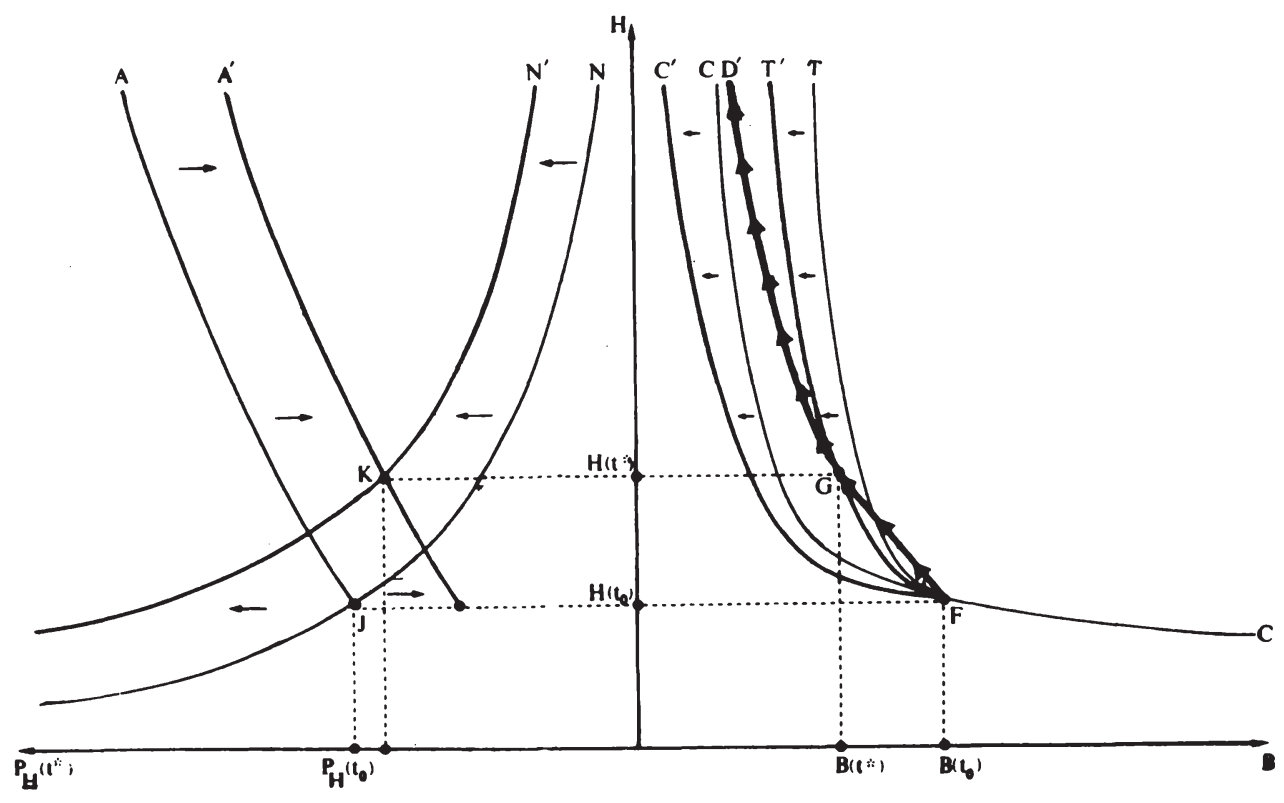

wicklungapfad FC gegen den Uhrzeigersinn in seine neue Position FC'

Wenn wir uns an die graphische Herleitung der Sprungkurve FT erinnern, so wissen wir, daB sich die FT-Kurve ebenfalls um $F$ nach links drehen muß - in Abb. 8 beschreibt FT' die neue Position dieser Kurve.

Darüberhinaus wurde in Kapitel II gezeigt (vgl. S.77), daß die Angebotskurve im linken Quadranten der $\mathrm{Abb} .7$ nach rechts rückt, wenn der Absolutwert des Differentialquotienten $\mathrm{dH} / \mathrm{dB}$ abnimmt. In dieser Verschiebung der Angebotskurve kommt die oben geschilderte Bereitschaft des Wohnungsinvestors zum Ausdruck, aufgrund der gesunkenen Bodenpreise, respektive Neubaukosten, seinen Bestand an Wohnflächeneinheiten in $t_{0}$ aufzustocken. 
tuber die steuerinduzierte Veränderung der Wachstumsrate des Häuserpreises wirkt sich die Besteuerung baureifer Grundstücke auch auf die Position der Nachfragekurve aus: löst man Bedingung (III.16b) nach $P_{H}$ auf und berücksichtigt die in (III.19) festgehaltene Tatsache, daß der Wert des im Wohnungssektor gebundenen Vermögens unter dem Einfluß einer steuer auf baureife Grundstücke schneller wächst, als es bei dem Verzicht auf die Erhebung einer solchen Steuer der Fall gewesen wäre, so erhält man als Gleichung für die neue Nachfragekurve

(III.20)

$$
P_{H}=\frac{m(a / H)}{(1-\beta) r+\alpha \delta-\beta \tau_{g r}} .
$$

(III.20) unterscheidet sich von der entsprechenden LaisserFaire-Funktion (II.83) durch den Grundsteuerterm $\beta \tau_{g r}$ : unter dem EinfluB der Besteuerung wird der Quotient auf der rechten seite des Gleichheitszeichens in (III.2O) größer, der Häuserpreis $\mathrm{P}_{\mathrm{H}}$ steigt folglich unter sonst gleichen Bedingungen - insbesondere bei gegebenem Wohnflächenbestand $H$; aus diesem Grunde rückt die Nachfragekurve in $\mathrm{Abb} .8$ aus ihrer Laisser-Faire-Position $\mathrm{N}$ in ihre neue Position $\mathrm{N}^{\prime}$; es wird sich für den vermögenbesitzenden und -bildenden Haushalt lohnen, sein Portefeuille zunächst zulasten der grundsteverpflichtigen Vermögensform "baureife Grundstücke" und zuqunsten der - in diesem Abschnitt als solche unterstellten - grundsteuerfreien Vermn̈qensform "bebaute Grundstücke" umzuschichten. Entsprechend wird sich der in seinem Auftrag handelnde Wohnungsinvestor verhalten.

Zunächst sinkende Baulandpreise und abnehmende Nutzungskosten des im Wohnungssektor gebundenen Vermögens haben zur Folge, daß in unmittelbarem AnschluB an die erstmalige Erhebung einer Steuer auf baureife Grundstücke zwischen tatsächlichem und gewünschtem Wohnflächenbestand eine Lücke im Umfang von $H(t *)-H\left(t_{0}\right)$ Wohnflächeneinheiten klafft. Diese Lücke wird - auch dies kann man Abb. 8 entnehmen - im Laufe der Kunstzeitphase $\left(t_{0}, t^{*}\right)$ geschlossen: Als unmittelbare Folge der Einführung einer steuer alleine auf den Bestand baureifer Grundstücke werden wir eine vorübergehende Mobilisierung von Bauland und einen ebenso vorübergehenden Boom am Markt für Wohnungsneubau beobachten können. 
Nach Abschluß der Kunstzeitphase bezeichnet der isoelastische Teilpfad GD den weiteren Entwicklungsprozess unserer Modellökonomie. Dieser ist dadurch gekennzeichnet daß

- das Niveau der Marktmieten $\mathrm{m}$, der Häuserpreis $\mathrm{P}_{\mathrm{H}}$ und der Bodenpreis $\mathrm{P}_{\mathrm{B}}$ nach anfänglichem Sinken nun schneller wachsen als in der steuerfreien welt; damit bewegen sich alle betrachteten Preise nach absehbarer zeit über ihrem Laisser-FaireNiveau;

- der Wohnflächenbestand $\mathrm{H}$ langsamer wächst und der Baubodenbestand B langsamer schrumpft als dies noch vor Erhebeung der Grundsteuer der Fall gewesen war. Deshalb werden trotz der anfänglichen Mobilisierung von Bauland und des daraufhin einsetzenden Neubaubooms jenseits eines absehbaren Zeitpunkts das wohnleistungsangebot geringer und der Baulandbestand gröBer als in der steuerfreien welt sein.

2.1.1.3. Die Besteuerung bebauter Grundstücke und die Planungsprobleme von Bodenmaklern und Wohnungsinvestoren

Nun muß aber nicht nur der Bodenmakler Grundsteuer zahlen, auch der Wohnungsinvestor wird zur Zahlung dieser steuer herangezogen; seine periodenbezogene Grundsteuerschuld beläuft sich auf einen D-Mark-Betrag in Höhe von

(III.21)

$$
\mathrm{T}_{g r}=\tau_{g r}{ }_{1} \mathrm{P}_{\mathrm{H}} \mathrm{H}
$$

In diesem Fall erhalten wir den Entnahmebetrag $A$ einer gegebenen Periode, indem wir von den Bruttomieteinnahmen $\mathrm{mH}$ zuzüglich der netto aufgenommenen Kredite $S$ die Ausgaben für werterhaltende Maßnahmen $E$, den $\mathrm{Z}$ insendienst $r D$, die Ausgaben für Neubauinvestitionen $F^{d} \varepsilon+P_{B} F^{d}$ und eben die Grundsteuerverpflichtungen aus (III.21) subtrahieren.

Ziel des Wohnungsinvestors ist es nun wiederum, durch eine optimale Planung der Nettoneuverschuldung, des Erhaltungsaufwandes, der Kapitalintensität der Bebauung und des Bodenverbrauchs den Barwert dieser Entnahmen $\mathrm{zu}$ maximieren. Da infolge der Annahme $P_{H}>1$ für die Kunstzeitvariable y zu jedem Zeitpunkt $t>t_{0} y=1 \mathrm{gilt}$, lautet sein Entscheidungsproblem 
folgerichtig

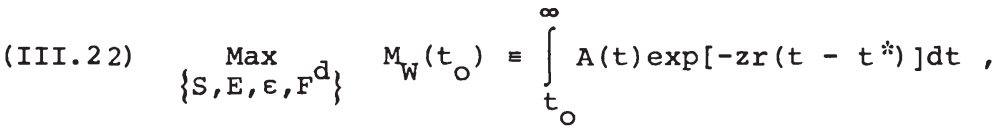

mit

(III.23)

$$
A=z\left(m H-E-r D-\tau_{g r} \gamma_{1} P_{H}^{H}\right)-F_{\varepsilon}^{d}-P_{B} F^{d}+s
$$

als periodenbezogene Entnahme. Als Restriktionen sind die folgenden Bedingungen $\mathrm{zu}$ beachten:

(II.2)

$$
H\left(t_{0}\right)>0,
$$

(II. 3)

$$
D\left(t_{0}\right) \leq 0,
$$

$$
O \leq E \leq \alpha \delta H,
$$

(II.10)

$$
\dot{\mathrm{H}}=\mathrm{F}^{\mathrm{d}} \varphi(\varepsilon)-z(\alpha \delta \mathrm{H}-\mathrm{E}),
$$

(II.14)

$$
S \leq P_{H}\left[F^{d} \varphi(\varepsilon)-z(\alpha \delta H-E)\right]+\dot{P}_{H} H
$$

(II. 16)

$$
\dot{\mathrm{D}}=\mathrm{s} \text {, }
$$

(II.17a)

$$
z= \begin{cases}0 & \text { für } t_{0} \leq t<t^{*} \\ 1 & \text { für } t \geq t^{*}\end{cases}
$$

In laufenden Werten ausgedrïckt lautet die Hamiltonfunktion für dieses Problem

(III. 24 )

$$
\begin{aligned}
H= & z\left(m H-E-r D-\tau_{g r} \gamma_{1} P_{H} H\right)-F^{d} \varepsilon-P_{B} F^{d}+S \\
& +P_{H}\left[F^{d} \varphi(\varepsilon)-z(\alpha \delta H-E)\right] \\
& +\lambda_{D} S .
\end{aligned}
$$


Wie man durch Differentiation von (III.24) nach den Kontrollen $S$ und $E$ leicht zeigen kann, werden durch die Besteuerung des Grundbesitzes weder die Laissez-Faire-Bedingung für die optimale Finanzierung noch die für den optimalen zeitpfad der werterhaltenden Arbeiten tangiert. Auf eine explizite Herleitung dieser Bedingungen kann an dieser Stelle deshalb verzichtet werden.

Durch Differentiation der Hamiltonfunktion aus (III.24) nach den Kontrollvariablen $\varepsilon$ und $F^{d}$ erhält man die notwendigen Bedingungen für die optimale Wahl der Kapitalintensität der Bebauung und des Bodenverbrauchs:

$$
\mathbf{P}_{H^{\varphi}} \varphi^{\prime}(\varepsilon)=1
$$

$$
\frac{\beta}{\alpha} \varepsilon\left\{\begin{array} { l } 
{ < \} } \\
{ = j }
\end{array} P _ { B } \rightarrow F ^ { d } \left\{\begin{array}{l}
=\} \\
\geq\}
\end{array}\right.\right. \text { O. }
$$

Schließlich erhält man die Bedingung für ein Portefeuillegleichgewicht, wenn man (III.24) nach der Zustandsvariablen $\mathrm{H}$ ableitet:

$$
\begin{aligned}
& \text { (III.27a) } \hat{\mathrm{P}}_{\mathrm{H}}=0 \text { für } t_{\mathrm{O}} \leq t<t^{*} \text {, } \\
& \text { (III.27b) } \quad \hat{\mathrm{P}}_{\mathrm{H}}=-\frac{\mathrm{m}}{\overline{\mathrm{P}}_{\mathrm{H}}}+r+\alpha \delta+\tau_{g r} \gamma_{1} \text { für } t \geq t^{*} \text {. }
\end{aligned}
$$

Die Bedingungen (III.25) und (III.26) sind uns aus der Analyse der Laissez-Faire-ర̌konomie bekannt. Bedingung (III.25) verlangt,daß im Investitonsoptimum das Wertgrenzprodukt des Gebäudekapitals gleich sein muß den Kosten der marginalen Bauleistungseinheit. Entsprechend besagt (III.26), daß es eine Neubautätigkeit nur dann geben wird $\left(F^{d}>0\right)$, wenn das Wertgrenzprodukt des Bodens $(\beta \varepsilon / \alpha)$ mit dem von den Bodenmaklern geforderten Bodenpreis übereinstimmt. Liegt die Zahlungsbereitschaft unter dem Angebotspreis, wird kein Bauland verkauft. Der Wohnungsneubau kommt dann zum Erliegen. Den Fall, in dem das wertgrenzprodukt den Bodenpreis übertrifft, hatten wir über die Existenzbedingung für eine Lösung des Planungsproblems des Wohnungsinvestors ausgeschlossen. 
(III.25) und (III.26) informieren uns auch darüber, daß die vom Wohnungsinvestor zu zahlende Grundsteuer einen unmittelbaren Einfluß hat weder auf die Wahl der Kapitalintensität der Bebauung noch auf die Entscheidung hinsichtlich des Bodenverbrauchs. Der Grund hierfür liegt in der Bemessungsgrundjage dieser Steuer: Anknüpfungspunkt ist der Grundstückswert, d.h. der wert des in bebauten Grundstücken gebundenen Vermögens, das infolge des putty-clay-Charakters der Produktionsfunktion für Wohnflächeneinheiten in einem nur indirekten Verhältnis zum Wert der eingesetzten Produktionsfaktoren steht.

Aus (III.2 5) und (III.26) lassen sich darüberhinaus wiederum die Wachstumseigenschaften des Häuserpreises und der Kapitalintensität der Bebauung im Gleichgewicht ableiten; aus (III.25) folgt

$$
\text { (III.28) } \quad \hat{\mathrm{P}}_{\mathrm{H}}=\beta \hat{\varepsilon},
$$

aus (III.26) erhält man die Beziehung

$$
\text { (III.29) } \quad \hat{\varepsilon}=\hat{\mathrm{P}}_{B} \text {. }
$$

Ein Blick auf die Bedingungen (III.28) und (III.29)zeigt, da die Besteuerung bebauter Grundstücke die Wachstumseigenschaften unserer Modellökonomie insoweit nicht beeinfluBt.

Die einzige Optimalitätsbedingung, in die die steuer auf bebaute Grundstücke unmittelbaren Eingang findet, ist (III.27). Aus der Analyse des Kapitels II wissen wir, daB diese Bedingung ein intersektorales Arbitragegleichgewicht beschreibt, indem sie fur das optimum die Ubereinstimmung der Nettorendite des in das Grundstück investierten Vermögens mit den user cost dieses Vermögens fordert. Und diese Nutzungskosten erhöhen sich nun infolge der Besteuerung des Grund und Bodens, wobei das AusmaB der Erhöhung von der Zeitnähe der Bewertung bebauter Grundstulcke abhängt. 
Spiegelt der Einheitswert den aktuellen Marktwert wider $\left(r_{1}=1\right)$, muß die Rendite $m / P_{H}$ der letzten in das bebaute Grundstück investierten D-Mark so groß sein, daß mit ihr nicht nur die auf diese D-Mark entfallenden Opportunitätskosten und abnutzungsbedingten Wertminderungen abzüglich der nachfragebedingten Wertsteigerungen gedeckt sind - auch die anteilige Grundsteuerbelastung mu $\beta$ aus dieser Rendite finanziert werden können. Es sollte keiner weiteren Erläuterung bedürfen, um zu erkennen, daß diese grundsteuerinduzierten Mehrkosten mit fallendem Bewertungsfaktor $\gamma_{1}$ sinken.

Damit entpuppt sich die Steuer auf bebaute Grundstücke als ein dem Wohnungsneubau auch kurzfristig abträgliches Element des bundesdeutschen Steuersystems - ganz im Gegensatz zur Besteuerung baureifer Grundstücke, die dem Wohnungssektor zu einem, alleräings vorübergehenden, Neubauboom verholfen hat. Für den Wohnungsinvestor bzw. für die Unternehmenseigner, in deren Interesse er agiert, wird es von Anfang an attraktiver sein, anstelle grundsteuerpflichtiger Wohnimmobilien verstärkt auf grundsteuerbefreite Finanzaktiva als Medium der Vermögensbildung zurückzugreifen.

\subsubsection{Die Besteuerung bebauter und unbebauter Grundstücke als konzeptionelle Einheit}

Entsprechend zweideutig sind auch die Aussagen über die kurzfristige Grundsteuerinzidenz, zu denen man gelangt, wenn man die Ergebnisse der Abschnitte III.2.1.1.1. und III.2.1.1.2. zusammenfaBt und die Besteuerung bebauter und baureifer Grundstücke als konzeptionelle Einheit betrachtet; substituiert man die Bedingung (III.19) in die Bedingung (III.27), so erhält man für die Rendite der letzten in den Wohnungssektor investierten D-Mark

(III.30)

$$
\frac{m}{P_{H}}=(1-\beta) r+\left(\gamma_{1}-\beta\right) \tau_{g r}+\alpha \delta
$$

Vergleicht man (III.30) mit der entsprechenden Laisser-FaireBedingung - aus (II.83) folgt in Verbindung mit (II.59) und (II.82) 
(III. 3 1)

$$
\frac{m}{P_{H}}=(1-\beta) r+\alpha \delta
$$

- so erkennt man, daß als Folge der Besteuerung des Grundvermögens die relative Vorteilhaftigkeit der Wohnungsinvestition verglichen mit der steuerfreien Situation kurzfristig sowohl zunehmen als auch abnehmen kann. Es hängt von den Unterschieden in der Bewertung bebauter und unbebauter Grundstücke $a b$, welche dieser beiden situationen tatsächlich eintritt.

Um diese dem Bewertungsrecht im Rahmen der Grundsteueranalyse zukommende Schlüsselrolle auch graphisch erhellen zu können, müssen wir uns in Erinnerung rufen, daß sich aus (III.30) die Funktion für die Nachfragekurven $\mathrm{N}^{\prime}, \mathrm{N}^{\prime}$ ' und $\mathrm{N}^{\prime \prime}$ ' in Abb.9 ableiten läßt; löst man (III.30) nach $P_{H}$ auf, so erhält man für den Graph dieser Kurven folgenden funktionalen zusammenhang:

$$
P_{H}=\frac{m(a / H)}{(1-\beta) r+\left(\gamma_{1}-\beta\right) \tau_{g r}+\alpha \delta} .
$$

Wie ein Blick auf (III.32) zeigt, fällt die Linksverschiebung der Nachfragekurve, die wir im Falle der alleinigen Besteuerung baureifer Grundstúcke in Abb. 8 beobachten konnten, unter dem Einfluß der zusätzlichen Besteuerung bebauter Grundstücke zumindest schwächer aus; für den Fall, daß bebaute Grundstücke relativ zeitnah bewertet werden - es gilt dann $\gamma_{1}>\beta-$, wird es anstatt $z u$ einer Linksverschiebung $z u$ einer Rechtsverschiebung der Nachfragekurve kommen.

Auf die Position der Angebotskurve nimmt die Besteuerung bebauter Grundstücke dagegen keinen EinfluB, ebensowenig auf die Lage der isoelastischen Entwicklungspfade und der Sprungkurve im rechten Quadranten der $\mathrm{Abb}$.7. Für die in $\mathrm{Abb} .9$ festgehaltenen Positionsänderungen dieser Kurven sind alleine die Reaktionen von Bodenmakler und Wohnungsinvestor auf die Erhebung einer steuer auf baureife Grundstücke ursächlich.

Den Fall einer relativ starken Unterbewertung bebauter Grundstücke - in diesem Fall gilt $\gamma_{1}<\beta$ - können wir rasch abhan- 
deln. Zwar fällt hier die Linksverschiebung der Nachfragekurve schwächer aus als im Fall der reinen Baulandsteuer - der nutzungskostensenkenden Wirkung grundsteuerbedingt erhöhter wertzuwächse des Wohnimmobilienvermögens steht nun die nutzungskostenerhöhende Wirkung der Belastung des Vermögens mit Grundsteuer-entgegen; wir werden aber nach wie vor feststellen können, daß eine Grundsteuer, verbunden mit dem hier unterstellten Bewertungsphänomen, einen den Wohnungsneubau kurzfristig belebenden Effekt zur Folge hat. Es wird also auch hier zum Zeitpunkt $t_{0} z u$ einem kurzfristigen Neubauboom kommen, nach dessen Abebben fur die weitere Entwicklung des systems dieselben wachstumseigenschaften Gültigkeit besitzen, die wir im Falle der reinen Baulandsteuer abgeleitet haben.

In Anbetracht dieses Ergebnisses ist es durchaus möglich, daB diejenigen Recht haben, welche die Auffassung vertreten, da $\beta$ das herrschende Grundsteuerrecht wirkungen zeigt, die vergleichbar sind mit denen der ehemaligen Grundsteuer $C$, deren Satz fur baureife Grundstücke auf das vier-bis sechsfache des Satzes der normalen Grundsteuer angehoben worden war, um einen Verkaufsdruck auf die Bodeneigentumer zu erzeugen. Wenn man bedenkt, daß diese steuer aufgrund verfassungsrechtlicher Bedenken wieder abgeschafft wurde, stellt sich die Frage, ob die bewertungsrechtliche Diskriminierung baureifen Bodens überhaupt ein mit dem Grundgesetz zu vereinbarendes Instrument einer zweifellos intendierten Forderung des Wohnungsneubaus darstellt.

Aber nicht nur, daß sich rechtliche Bedenken hinsichtlich der Besonderheiten der praktizierten Besteuerung des Grund und Bodens anmelden - es ist auch ganz und gar nicht sicher, da $B$ sich die mit der steuer kurzfristig angestrebten ziele überhaupt realisieren lassen.

Denn aller bewertungsrechtlichen Diskriminierung zum Trotz ist es durchaus möglich, daß die Diskriminierung $z u$ schwach ausfällt und der Grundsteuerterm $\left(\gamma_{1}-\beta\right) \tau_{g r}$ in der Nachfragefunktion (III.32) ein positives Vorzeichen annimmt. In diesem 


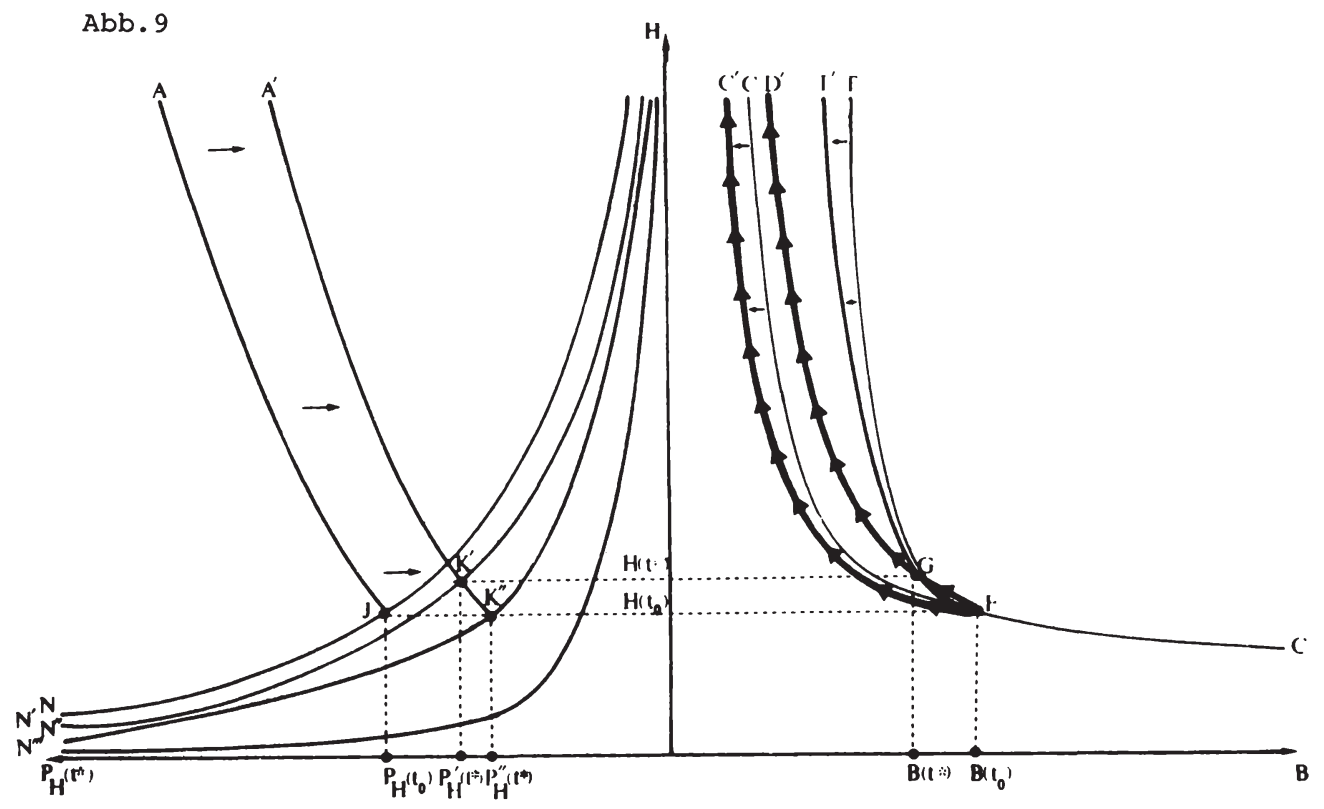

Falle verliert der Quotient auf der rechten Seite des Gleichheitszeichens von (III.32) grundsteuerbedingt an Wert und die Nachfragekurve wandert nach rechts. In Abb. 9 ist dieser Fall graphisch wiedergegeben.

Wie das System nun in unmittelbarem AnschluB an die erstmalige Erhebung der steuer reagiert, hängt vom relativen Ausmaß der Verschiebung von Angebots- und Nachfragekurve ab. Es sind hierbei drei Fälle denkbar, die ihr graphisches Pendant in den in Abb.9 so bezeichneten Nachfragekurven $\mathrm{N}^{\prime}, \mathrm{N}^{\prime}$ ' und $\mathrm{N}^{\prime}$ '' besitzen.

Fall 1: Entspricht im Ausgangspunkt $t_{0}$ die Nachfrage nach Wohnleistungen dem Angebot, so kann es im AnschluB an die Einführung einer Grundsteuer mit relativ zeitnaher Bewertung zu einem Neubauboom am Wohnungsbestandsmarkt kommen. Die grundsteuerbe- 
dingte Erhöhung der Nutzungskosten des im Wohnungsbestand gebundenen Vermögens reicht in diesem Falle nicht aus, den expansiven Effekt zu neutralisieren, der von den im Zuge der ebenfalls grundsteuerbedingten Baulandmobilisierung sinkenden Wohnungsneubaukosten ausgeht. In unserer Graphik wäre diese Situation dănn gegeben, wenn die Nachfragekurve nach Steuer die Position $\mathrm{N}^{\prime}$ einnehmen würde. In dieser Situation werden die Wohnungsinvestoren bestrebt sein, ihren Bestand an Wohnflächeneinheiten so schnell als möglich aufzustocken. Das System wird zum Zeitpunkt $t_{0}$ von $F$ nach G springen - die Bestandsanpassung von $H\left(t_{0}\right)$ auf $H\left(t^{*}\right)$ vollzieht sich innerhalb der Kunstzeitphase -, um sich danach entlang der Kurve GD' weiterzubewegen.

Fall 2: Es ist aber auch der Fall denkbar, daß die Besteuerung des Grund und Bodens einen Angebotsüberschuß am Markt für Wohnleistungen im unmittelbaren Gefolge hat; die Kurve N' ' spiegelt dann die aktuelle Nachfragesituation wider. Der Wohnungsinvestor ist nun nicht mehr in der Lage, den vorhandenen Wohnflächenbestand kostendeckend $z u$ einem Preis zu vermieten, bei dem die privaten Haushalte willens wären, das aus dem bestehenden Bestand fließende Wohnleistungsangebot auch in vollem Umfang nachzufragen. Andererseits kann die vorhandene Ubberkapazität im Bestand infolge der Irreversibilität der Bebauung nicht abgebaut werden. Die einzig sinnvolle Handlungsweise des Wohnungsinvestors besteht nun darin, den Wohnungsneubau ceteris paribus solange einzustellen, bis sich die Nachfragekurve infolge der im Zeitablauf permanent ansteigenden Wohnleistungsnachfrage soweit nach links verschoben hat, daß sie die Angebotskurve $A^{\prime}$ schneidet. Die ökonomie verharrt während dieser Ubergangsphase in Punkt $\mathrm{F}$ und entwickelt sich danach entlang der Kurve FC' weiter.

Fall 3: Wenn dagegen auch nach Einführung der Grundsteuer Angebot und Nachfrage auf dem Mietwohnungsmarkt übereinstimmen diesem Fall entspräche die Nachfragekurve N'' - wird man vergeblich auf irgendwelche Anpassungsreaktionen am Markt für Wohnungsneubau warten. Der Entwicklungspfad der ókonomie wird auch in diesem Fall durch die Kurve FC' beschrieben. 
Letzten Endes lassen sich also keine exakten Aussagen über die kurzfristigen Reaktionen der Wohnungs- und Baulandmärkte auf die Einführung einer Grundsteuer bundesdeutschen Rechts ableiten; wie groß die Unterschiede in der zeitnähe der Bewertung von baureifen und bebauten Grundstücken letztendlich sein müssen, damit Bauland - wie vom Gesetzgeber gewünscht - auch tatsächlich beschleunigt bereitgestellt und bebaut wird, läßt sich ohne Kenntnis gewisser produktionstechnischer Sachverhalte (wie groß ist B?) nicht sagen.

So unterschiedlich die denkbaren Reaktionen unserer Modellökonomie in der kurzen Frist sind, so eindeutig läBt sich die langfristige Inzidenz der bundesdeutschen Grundsteuer beschreiben - denn sie ist in ihrer Qualität unabhängig von der Willkür des Bewertungsrechts und der Erhebungspraxis.

Unter dem Einfluß der Grundsteuer werden der Mietzins $m$, der Häuserpreis $P_{H}$, der Bodenpreis $P_{B}$ und auch die Kapitalintensität $\varepsilon$ der Bebauung mit einer gröBeren Rate wachsen, als dies in einer Laisser-Faire-Konkurrenzökonomie der Fall wäre. Die Folge davon ist, daß alle preise, selbst wenn sie anfänglich sinken sollten, in absehbarer zeit über ihrem Laisser-Faire Niveau liegen werden. Dagegen wird sich das wachstum des wohnflächenbestandes $\mathrm{H}$ ebensa wie das Schrumpfen des Bodenbestandes $B$ verlangsamen.

Aus diesem Grunde wird man all jene enttäuschen müssen, die sich von der grundsteuerlichen Diskriminierung baureifer Grundstücke eine beschleunigte Bereitstellung von Bauland bei gleichzeitig sinkenden Bodenpreisen und auf diese Weise eine "Wiederherstellung der Funktionsfähigkeit des Bodenmarktes" erhoffen." Bei einer solch undifferenzierten Betrachtungsweise wird übersehen, daß es gerade solche Eingriffe sein können, die die Funktionstüchtigkeit des Bodenmarktes beeinträchtigen. Im Lichte unserer Analyse geradezu paradox mutet es an, wenn auf der einen Seite beklagt wird, daß der Bodenmarkt infolge "zu hoher" Boden45) ERNST(1971), S.25. 
preise zu einem "FaB ohne Boden" wird, im gleichen Atemzug aber staatliche Eingriffe gefordert werden, die die "Monopolmacht" der Bodenbesitzer brechen, das Bodenangebot erhöhen und die Bodenpreise senken sollen 46). Denn durch eine solche Politik würden die beklagten Probleme nicht gelöst, sondern in verschärfter Form in die zukunft übertragen werden: wer heute zusätzliches Bauland mobilisiert und dadurch das Angebot an Bauland in zukünftigen Perioden verknappt, darf sich nicht wundern, wenn die Bodenpreisentwicklung in diesen Perioden eine noch kräftigere Tendenz nach oben aufweist.

Auch die Vorstellung, daß eine "soziale Bodenpolitik" in der beschleunigten Bereitstellung von Bauland gipfeln mulsse ${ }^{47}$ ), wird durch unsere Analyse ad absurdum geführt. Da der Wohnflächenbestand grundsteuerbedingt mit einer geringeren Rate wächst als in der steuerfreien Welt und es deshalb selbst nach einem - sich keineswegs zwangsläufig einstellenden grundsteuerinduzierten Neubauboom nur eine Frage der zeit ist, bis sich das Wohnleistungsangebot unterhalb seines Laisser-FaireNiveaus bewegt, würde das auf dem Wege einer Baulandmobilisierung letztendlich angestrebte $\mathrm{ziel}$ der Verbesserung der Wohnversorgung durch eine solche Politik konterkariert werden.

Die Tatsache, daß Baulandpreise "zu hoch" sind - schon alleine diese Einschätzung ist mißverständlich, suggeriert sie doch die Existenz eines "gerechten" Bodenpreises - muß ihre Ursache nicht unbedingt und ausschließlich in einer spekulativen zurückhaltung von Bauland haben, "zu hohe" Bodenpreise können auch die Folge einer in der Vergangenheit zu umfangreichen Bereitstellung von Bauland sein. Damit aber erweist sich die bewuBte steuerliche Diskriminierung von Baulandeigentümern unter Umständen als ein zweischneidiges schwert und die übliche mechanistische Betrachtungsweise des Bodenmarktes - sinkende Preise bei steigendem Angebot und gegebener Nachfrage - als eine zu kurzsichtige.

46) PFEIFFER(1982), S.63.

47) SCHNEIDER, O. (1982), S.44 . 
2.1.2. Die Bodenwertsteuer - die beste Lösung?

Wie aus den vorangegangenen Ausführungen deutlich hervorgeht, trägt die bundesdeutsche Grundsteuer ihren Namen zu unrecht; denn tatsächlich haben wir es hier mit einem Komplex verschiedener Steuerarten zu tun: unter dem Oberbegriff "Grundsteuer" subsummiert sind

- eine Landwirtschaftsteuer (Grundsteuer A),

- eine Gebäudeertragsteuer bei bebauten Grundstücken und

- eine Grundwertsteuer in Form einer Grundvermögensteuer bei unbebauten und nicht land- oder forstwirtschaftlich genutzten Grundstücken. ${ }^{48)}$

Angesichts dieser steuersystematisch wenig überzeugenden Struktur der deutschen Grundsteuer stehen denn auch weniger die zuvor beschriebenen intertemporalen Verzerrungen im Mittelpunkt der Kritik am geltenden Grundsteuerrecht, vielmehr ist es die oben angesprochene Verschiedenheit der Anknüpfungspunkte, die den meisten Kritikern der größte Dorn im Auge ist.

Insbesondere wird eine Benachteiligung der Eigentümer bebauter Grundstücke gegenüber den Eigentümern baureifer Grundstücke darin gesehen, daß bei den Erstgenannten nicht nur der Grund und Boden, sondern auch das darauf stehende Gebäude inklusive Außenanlagen der Steuer unterworfen werden ${ }^{49}$ ? Vor allem aus diesem Grund wird in der einschlägigen steuertheoretischen Literatur in schöner Regelmäßigkeit eine Reform des geltenden Grundsteuerrechts gefordert, deren Kern eine Besteuerungsform des Grund und Bodens sein soll, die allein den Bodenwert ohne den Wert des aufstehenden Gebäudes und der Außenanlagen trifft.

Das Spektrum der Erwartungen, die hinsichtlich einer Bodenwertsteuer gehegt werden, ist außerordentlich umfangreich und reicht von einer Mobilisierung des Baulandangebots [z.B. BOHNSACK(1967)] über die Neutralität dieser Steuer [z.B. RICARDO(1905), BESSERER(1984), SINN(1984)] bis hin zu einer verstärkten zurückhaltung baureifer Grundstücke [z.B. FRIAUF/RISSE/WINTERS (1978)]. Diese offensichtlichen Widersprüche machen es reizvoll 48)BICKEL (1956), S.431.

49) Eine Literaturübersicht zu diesem Thema geben FRIAUF/RISSE/WIIIERS(1978) . 
zu untersuchen, welchen Einfluß eine Bodenwertsteuer auf die Planungen des Wohnungsinvestors nun tatsächlich ausübt; da die praktizierte Besteuerung baureifer Grundstücke mit dem Konzept einer Bodenwertsteuer übereinstimmt, kann man davon ausgehen, daB der Bodenmakler seinen intertemporalen Bodenangebotsplan bei einem Wechsel von der Grundsteuer geltenden Rechts auf eine Bodenwertsteuer nicht verändert.

Das erste Problem, das sich bei der Implementierung einer Bodenwertsteuer stellt, ist die Wahl des adäquaten Bewertungsmaßstabs für den Bodenanteil bebauter Grundstücke.

Nicht selten wird gefordert, auch der Bewertung dieses Anteils den aktuellen Marktpreis $P_{B}$ baureifen Bodens zugrundezulegen ${ }^{50}$ ! Wie sich leicht zeigen läßt, wäre diese wahl aber verfehlt. Würde man diesem Vorschlag nämlich folgen, so erhielte man als Bemessungsgrundlage für die Besteuerung bebauter Grundstücke den "Bodenwert"

$$
\left(\operatorname{III.33)} \quad \mathrm{BW}=\mathrm{P}_{\mathrm{B}} \mathrm{B}^{*},\right.
$$

wobei $B^{*}$ für den Bodenanteil am Gesamtbestand bebauter Grundstücke steht. In das Entscheidungsproblem des Wohnungsinvestors müßte $\mathrm{B}^{*}$ als weitere Zustandsvariable Eingang finden. Insbesondere müßte dann für den Bodenwert auch die Transversalitätsbedingung

$$
\lim _{t \rightarrow \infty}\left[P_{B}(t) B^{*}(t) \exp \left[-\operatorname{zr}\left(t-t_{0}\right)\right]\right]=0
$$

erfüllt sein - denn nur dann wird der Barwert des Aufkommens der Bodenwertsteuer ein endlicher Wert sein und folglich auch eine Lösung des Planungsproblems des Wohnungsinvestors existieren. Bedingung (III. 34) ist nun aber gerade dann nicht erfüllt, wenn $P_{B}$ als Bewertungsmaßstab verwendet wird. Dies wird deutlich, wenn man anstelle von (III.34) die äqivalente Formulierung

$$
\text { (III.35) } \quad \lim _{t \rightarrow \infty}\left[\hat{P}_{B}(t)+\hat{B}^{*}(t)-r\right]<0
$$

50) Vgl. z.B. BOHNSACK (1967), S.47. 
verwendet. Aus der Analyse des Entscheidungsproblems des repräsentativen Bodenmaklers wissen wir, daB die Bodenpreissteigerungsrate unter dem EinfluB der Bodenwertsteuer den Wert $\hat{\mathrm{P}}_{\mathrm{B}}=r+\tau_{g r}$ annimmt. Berücksichtigt man ferner, daB infolge der Irreversibilität der Bebauung und der Tatsache, daß zumindest jenseits einer möglichen Ubergangsphase in jeder Periode neue Wohnungen errichtet werden, $\hat{B}^{*}>0 \mathrm{gilt}$, so wird augenscheinlich, daß Bedingung (III.35) bei einer Verwendung von $P_{B}$ als Bewertungsmaßstab für den Bodenanteil bebauter Grundstücke verletzt werden würde. Der Barwert der Grundsteuerbelastung nähme für den Wohnungsinvestor eine nicht mehr vorstellbare Höhe an, eine Wohnungsinvestition wäre deshalb auch nicht mehr rentabel.

Die ökonomische Intuition, die sich hinter dieser formalen Aussage verbirgt,ist die folgende: offensichtlich ist der Bewertungsmaßstab $P_{B}$ ein willkürlicher Bewertungsmaßstab, der mit dem Bodenwert eines bebauten Grundstücks in keinem Zusammenhang steht. Besonders deutlich wird dies, wenn man sich liberlegt, welchen Preis in unserem Modell der Wohnungsinvestor netto erzielen könnte, wenn er bebauten Grund und Boden auf dem Markt für baureifes Land anbieten wollte. Der erzielbare Bruttopreis läge für die einzelne Bodenflächeneinheit sicherlich bei $P_{B}$. Gegenrechnen müBte unser Wohnungsinvestor nun allerdings die Kosten, die ihm durch die Umwidmung von bebautem in unbebauten Grund und Boden entstünden; dieser Betrag setzt sich zusammen aus dem Barwert der durch den Verkauf entgangenen Nettomieterträge zukünftiger Perioden und den Abrißkosten für das Gebäude.

Nur wenn die Differenz aus dem erzielbaren Bruttobodenpreis $P_{B}$ einerseits und den Umwidmungskosten andererseits nicht negativ ist, wird man die Umwidmung unter ökonomischen Gesichtspunkten als rational bezeichnen können.

Für unsere Modellüberlegungen haben wir in Kapital II prohibitiv hohe AbriBkosten unterstellt. Entsprechend unmöglich wird es deshalb sein, in unserem Modell für den Bodenanteil bebauter Grundstücke einen aus Angebot und Nachfrage abgeleiteten Bewertungs- 
maßstab zu finden.

Doch selbst wenn man die Annahme prohibitiv hoher AbriBkosten aufgibt und die Umwidmung bebauter Grundstücke in unbebaute zuläßt, wäre es nur zum Abribzeitpunkt möglich, den Bodenanteil eines bebauten Grundstücks mit Hilfe des aktuellen Preises für Bauland korrekt zu bewerten. Allerdings entspräche der ökonomische Wert des Grundstücks dann nicht dem Produkt aus Bodenpreis und Grundstücksfläche, abzuziehen von diesem Produkt wären vielmehr noch die Kosten für den AbriB aufstehender Gebäude. Solange es dem Wohnungsinvestor aber nicht vorteilhaft erscheint, aufstehende Gebäude abreißen zu lassen, muß eine Wertermittlung unter Zuhilfenahme des Baulandpreises $P_{B}$ selbst unter Berücksichtigung von Abrißkosten zwangsläufig $2 u$ falschen Ergebnissen führen.

Wie sollte nun aber der korrekte Bewertungsmaßstab für bebaute Grundstücke aussehen? Wie die obigen Ausführungen gezeigt haben, gibt es auf diese Frage im Grunde genommen keine Antwort, da der Bodenanteil im ökonomischen Sinne ebensowenig einen eigenen Wert besitzt wie das Gebäude. Der Grund hierfür ist in dem putty-clay-Charakter der Produktionsfunktion für Wohnflächeneinheiten zu sehen: zu dem zeitpunkt, zu dem Boden und Bauleistungen $z u$ Wohnflächeneinheiten kombiniert werden, verlieren beide Produktionsfaktoren ihren spezifischen Charakter - im ökonomischen sinne bewertbar ist dann nur noch die Einheit "bebautes Grundstück".

Trotzdem sprechen sich seit den Anfängen der Steuertheorie ökonomen immer wieder dafür aus, eine Besteuerung des Bodens an der Nettorente auszurichten, die dieser Boden abwirft. So war schon RICARDO der Uberzeugung, daB eine Grundsteuer, die der durchschnittlichen Grundrente entsprechend erhoben wird und sich nach jeder ihrer Veränderungen richtet, für die Gemeinschaft vorteilhaft sei, weil sie einerseits dem staat steuern verschaffe, andererseits aber die Handlungsweise von Pächtern und Verpächtern unbeeinflußt lasse ${ }^{51}$, in unserem sinne also neutral ist. 51) RICARDO(1905), S. 177 ff. 
Nach RICARDOs Meinung dürfe eine solche Steuer auch nicht den Ertrag des Kapitals belasten, "das des bloßen Profits halber in den Boden gesteckt wird."

Wie läßt sich die Bemessungsgrundlage einer Bodenwertsteuer RICARDOscher Provenienz nun aber in termini unserer Modellvariablen ausdrücken? Zunächst ist anzumerken, daß die Forderung, die Grundsteuer an der Grundrente auszurichten, nicht gleichbedeutend sein kann mit dem Verlangen, daß sich die Bodenwertsteuer an den periodenbezogenen Nettoerträgen des betreffenden Bodens orientieren solle - es sei denn, man unterstellt RICARDO, $\mathrm{da} \beta$ er zwar von einer Grundsteuer gesprochen, tatsächlich aber eine Einkommensteuer im Sinn hatte. Ergebnis der einzig sinnvollen Interpretation der Ausführungen RICARDOs ist die Forderung, daß die Bodenwertsteuer die durchschnittliche Rente treffen soll, die das Grundstück bei einer ordnungsgemäßen Bewirtschaftung abwirft - abzüglich des durchschnittlichen Ertrags, der auf das in dem aufstehenden Gebäude gebundene Vermögen entfällt.

Wie man sich anhand einer einfachen Arbitrageüberlegung leicht klarmachen kann, entspricht die durchschnittliche Grundstücksrente dem Produkt des Kapitalmarktzinses $r$ und des Ertragswertes des Grundstücks; der Wohnungsinvestor steht nämlich am Ende jeder Periode vor der Wahl, entweder seinen gesamten Häuserbestand zu verkaufen und den dadurch erzielbaren Verkaufserlös $\mathrm{P}_{\mathrm{H}} \mathrm{H}$ am Kapitalmarkt anzulegen oder auf den verkauf $\mathrm{z}$ u verzichten und den Wohnflächenbestand $\mathrm{H}$ zum Preis $\mathrm{m}$ pro Wohnflächeneinheit $z u$ vermieten. Im Gleichgewicht muß offensichtlich gelten, daß er zwischen beiden Wahlmöglichkeiten indifferent ist. Dies wird aber nur dann der Fall sein, wenn der aus der

52) Von RICARDO inspirieren lieB sich wohl auch LUBAHN, der zur Bewertung bebauter Grundstücke den Preis der gesamten Bodennutzung, vermindert um die 'Zinsen des in Anlagen investierten Kapitals und die Absetzungen für Abnutzung vorschlug;-vgl. LUBAHN, zitiert in FRIAUF/RISSE/WINIERS (1978), S.103. Ähnliche, z.T. auch konkretere Vorschläge finden sich bei NEIL-BREUNING. 
Vermietung des Grundstücks resultierende Gewinn gleich ist dem Ertrag $\mathrm{rP}_{\mathrm{H}} \mathrm{H}$ der alternativen Kapitalmarktanlage; $r \mathrm{P}_{\mathrm{H}} \mathrm{H}$ ist demnach dle Ausgangsgröße zur Berechnung der Grundstücksrente. RICARDO folgend muß nun noch der Ertrag des Kapitals steuerfrei bleiben, "das des bloßen Profits halber in den Boden gesteckt wird". Uber die Höhe dieser Opportunitätskosten gibt uns das Produkt aus Marktzinssatz und Ausgaben für Gebäudekapital FE bzw. - wenn man den gesamten Gebäudekapitalstock K betrachtet das Produkt aus Marktzinssatz $r$ und Gebäudekapitalstock $K$ Auskunft. Die Begründung hierfür folgt wiederum aus einer Arbitrageüberlegung. Anstatt den Geldbetrag $\mathrm{K}$ in Gebäude $\mathrm{zu}$ investieren, hätten die Kapitalanleger dafür auch Finanzaktiva erwerben können, die ihnen in jeder Periode zu zinserträgen in Höhe von rK D-Mark verholfen hätten. Im Gleichgewicht muß nun wiederum die Bedingung erfüllt sein, daß beide Investitionsalternativen den gleichen Ertrag erwirtschaften.

Bezeichnet $\tau_{g r}^{*}$ den betreffenden Grundsteuersatz, so erhalten wir als Aufkommen der von RICARDo vorgeschlagenen Bodenwertsteuer

(III. 36)

$$
T_{g r}=\tau_{g r}^{*} r\left(P_{H} H-K\right), \quad 0<\tau_{g r}^{*}<1 .
$$

Aus Vereinfachungsgründen wollen wir anstelle des Produkts $\tau_{g r}^{*} r$ aus Gleichung (III. 36) den Grundsteuersatz $\tau_{g r}$ wählen; mit (III. 37) $\quad \tau_{g r}=\tau_{g r}^{*} r$

ist sichergestellt, daB die $z u$ zahlende Grundsteuer, wie vom Gesetzgeber intendiert, bei ordnungsgemäßer Bewirtschaftung des Grundstücks nicht aus der Vermögenssubstanz heraus, sondern aus den laufenden Erträgen des Grundvermögens finanziert werden kann.

In Gestalt des Gebäudekapitalstocks $\mathrm{K}$ taucht nun eine weitere Zustandsvariable im Entscheidungsproblem des Wohnungsinvestors auf. Wenn man sich daran erinnert, daß der Gebäudekapitalstock in jeder Periode abnutzungsbedingt im Ausmaß von $6 \mathrm{~K}$ Wohnflächen- 
einheiten an Substanz verliert, diese Substanzverluste ihrerseits aber durch entsprechende Renovierungsarbeiten $E$ wieder aufgeholt werden, so gilt für die zustandsvariable $\mathrm{K}$ folgerichtig die Bewegungsgleichung

$(\operatorname{III.38)} \quad \dot{K}=F \varepsilon-z(\delta K-E)$.

Wenn man nun die Bodenwertsteuer aus (III.36) unter Berücksichtigung von (III.38) in das Planungsproblem des Wohnungsinvestors integriert, erhält man für dieses

(III.39) $\left\{S_{S, E, \varepsilon, F}{ }^{M a x} M_{W}\left(t_{0}\right)=\int_{t_{0}}^{\infty} A(t) \exp [-z r(t-t *)] d t\right.$

unter den Bedingungen (II.2), (II.3), (II.9)', (II.10), (II.14), (II.16), (II.17a), (III.38) und

(III. 40)

$$
A=z\left[m H-E-r D-\tau_{g r}\left(P_{H} H-K\right)\right]+S-F E-P_{B} F^{d}
$$

Entsprechend lautet die in laufenden Werten definierte Hamiltonfunktion für das Planungsproblem aus (III.39)

$$
\begin{aligned}
H= & z\left[m_{H}-E-r D-\tau_{g r}\left(P_{H} H-K\right)\right]+S-F^{d} \varepsilon-P_{B} F^{d} \\
& +P_{H}\left[F^{d} \varphi(\varepsilon)-z a(\delta H-E)\right] \\
& +\lambda_{D} S_{W} \\
& +\lambda_{K}\left[F^{d} \varepsilon-z(\delta K-E)\right] ;
\end{aligned}
$$

$\lambda_{K}$ ist der Schattenpreis des Gebäudekapitalstocks im Planungsproblem des Wohnungsinvestors. Durch Differentiation von (III.41) nach den Kontroll- und zustandsvariablen erhält man die für ein Optimum notwendigen Bedingungen ${ }^{53}$ ).

53) Da auch die Bodenwertsteuer keinen Einfluß hat auf die Laisser-FairePfade der Nettoneuverschuldung und des Erhaltungsaufwandes, wird an dieser Stelle auf eine eingehendere Betrachtung der entsprechenden optimalitätsbedingungen verzichtet. 
Aus $\partial H / \partial \varepsilon=0$ folgt die Bedingung für den optimalen Einsatz von Bauleistungen bei der Produktion von Wohnflächeneinheiten:

$$
\mathrm{P}_{\mathrm{H}} \varphi^{\prime}(\varepsilon)+\lambda_{\mathrm{K}}=1
$$

Im Unterschied zu Bedingung (II.35) der Laisser-Faire-Situation und $\mathrm{zu}$ Bedingung (III.25) der Grundsteueranalyse taucht in (II.42) zusätzlich noch der Schattenpreis des Gebäudekapitalstocks auf; für diesen gilt

$$
\begin{aligned}
\lambda_{K}\left(t_{0}\right) \equiv \frac{\partial M_{W}\left(t_{0}\right)}{\partial K\left(t_{0}\right)} & =\int_{t_{0}}^{\infty} \frac{\partial K(t)}{\partial K\left(t_{0}\right)} \tau_{g r} \exp \left[-z r\left(t-t^{*}\right)\right] d t \\
& =\int_{t_{0}}^{\infty} \tau_{0 r} \exp \left[-z(r+\delta)\left(t-t^{*}\right)\right] d t \\
& =\frac{\tau_{g r}}{r+\delta} ;
\end{aligned}
$$

$\lambda_{K}$ spiegelt die Tatsache wider, daß bei gegebenem Wert des Wohnflächenbestandes der Marktwert des Wohnungsunternehmens umso höher ist, je gröBer der Anteil des grundsteuerfreien Wohnungskapitalbestandes ist. Dadurch, daß der Einsatz des Produktionsfaktors Boden bei der Erstellung von Wohnflächeneinheiten durch die Erhebung einer Bodenwertsteuer diskriminiert wird, wird es für den Wohnungsinvestor lohnend, bei der Produktion von Wohnraum vergleichsweise mehr Einheiten des Produktionsfaktors Bauleistungen einzusetzen. GemäB (II.43) ist $\lambda_{K}$ der Barwert der Grundsteuerersparnis, die aus einer marginalen Erhöhung der Kapitalintensität der Bebauung resultiert. Folgerichtig werden im Gleichgewicht nach steuer unter sonst gleichen Bedingungen die Anforderungen an die Grenzproduktivität des Produktionsfaktors "Bauleistungen" geringer ausfallen, verglichen mit den Anforderungen, die das geltende Grundsteuerrecht an das Wertgrenzprodukt dieses Faktors stellt. 
Für den Bodenverbrauch im neuen Gleichgewicht errechnet man aus $\partial H / \partial F^{d}$ die Bedingung

$$
\text { (III.44) } \quad \frac{\beta}{\alpha}\left(1-\lambda_{K}\right) \in\left\{\begin{array}{l}
< \\
=
\end{array}\right\} P_{B} \Rightarrow F^{d}\left\{\begin{array}{l}
= \\
\geq
\end{array}\right\} 0
$$

In (III.44) wird verlangt, $\mathrm{da} \beta$ im Gleichgewicht, so es eine Neubautätigkeit geben soll, das Wertgrenzprodukt des Bodens bei der Produktion von Wohnflächeneinheiten übereinstimmen muß mit dem in Rechnung gestellten Bodenpreis. Und aus (III.44) geht auch hervor, daß infolge der grundsteuerlichen Diskriminierung von Bauland die Wohnungsinvestoren als Reaktion auf die Erhebung einer Bodenwertsteuer die Kapitalintensität der Bebauung erhöhen, wenn die Bodenmakler nicht bereit sind, niedrigere Bodenpreise $\mathrm{zu}$ akzeptieren.

Differenziert man (III.41) nach der Zustandsvariablen $\mathrm{H}$, so resultieren hieraus die Bedingungen

$$
\begin{array}{lll}
\text { (III.45a) } & \hat{\mathrm{P}}_{\mathrm{H}}=0 \quad \text { für } t_{\mathrm{O}} \leq t<t *, \\
\text { (III.45b) } & \hat{\mathrm{P}}_{\mathrm{H}}=-\frac{m}{\mathrm{P}_{H}}+r+\alpha \delta+\tau_{\text {gr }} \text { für } t \geq t * .
\end{array}
$$

Offensichtlich nehmen die Nutzungskosten des im Wohnungssektor gebundenen Vermögens unter dem Regime einer idealtypischen Bodenwertsteuer im gleichen Umfang $\mathrm{zu}$ wie unter dem Regime einer Grundsteuer geltenden Rechts bei zeitnaher Bewertung des Grundstückvermögens (in (III.31b) gilt dann $\gamma_{1}=1$ ). Dieses Ergebnis wird verständlich, wenn man bedenkt, dāß eine Bodenwertsteuer steueranalytisch gleichzusetzen ist mit einer steuer auf den Ertragswert des Grundstücks abzüglich einer öffentlichen Subvention auf die reinen Bauleistungskosten der Wohnungsinvestition.

Neben diesen Ergebnissen ist für uns aber auch die Antwort auf die Frage von Interesse, mit welchen Reaktionen des Wohnungsund des Baulandmarktes man bei einem Ubergang von der Grundsteuer geltenden Rechts auf die auch im politischen Raum propagierte Bodenwertsteuer rechnen muß. 
Den Schlüssel $z u$ dieser Antwort finden wir in der Gleichgewichtsbedingung (III.42) und in der Rolle, die diese Bedingung bei der Formulierung der Angebotsfunktion für Wohnleistungseinheiten spielt: gemäB (II.79) gilt für die Angebotsfunktion die allgemeine Beziehung

$$
H\left(t^{*}\right)=\Phi\{\Phi[\varepsilon(t *)]\}_{3} \varphi^{\prime}, \Phi^{\prime}>0,
$$

die sich unter Berücksichtigung der Inversen von (III.42) umformen läßt $\mathrm{zu}$

$$
H\left(t^{*}\right)=\Phi\left\{\varphi\left[\varphi^{\prime-1}\left(\frac{1-\lambda_{K}}{P_{H}\left(t^{*}\right)}\right)\right]\right\}
$$

mit $\partial H\left(t^{*}\right) / \partial P_{H}\left(t^{*}\right), \partial H\left(t^{*}\right) / \partial \lambda_{K}>0$.

Vergleicht man die Angebotsfunktion in (III.46) mit der korrespondierenden, im Rahmen der Analyse des geltenden Grundsteuerrechts abgeleiteten Funktion, so wird offenkundig, daB die Angebotskurve $A^{\prime}$ in Abb. 9 nach rechts rücken würde, wenn sich der Gesetzgeber in $t_{0}$ dazu entschlösse, die Grundsteuer geltenden Rechts durch eine Bodenwertsteuer zu ersetzen: da die Bodenwertsteuer im Gegensatz zur geltenden Grundsteuer den Gebäudekapitalbestand nicht belastet, würden sich für den Wohnungsinvestor im Zuge dieser Steuerreform die Kosten des Wohnungsneubaus verringern; Folge dieser Kostensenkung würde sein, daB er im AnschluB an die Steuerreform bereit wäre, seine Neubautätigkeit $z u$ intensivieren und seinen Wohnflächenbestand aufzustocken.

Da der Bodenmakler seine Bodenverkaufsplanung im AnschluB an die steuerreform nicht verändern wird, kommt in dieser Verschiebung zugleich auch die gesuchte differentielle Inzidenz zwischen den beiden bisher betrachteten Besteuerungsformen des Grundvermögens zum Ausdruck. In Abb. 10 wird die dynamische Inzidenz dieser Grundsteuerreform graphisch festgehalten: 
$\mathrm{Abb} .10$

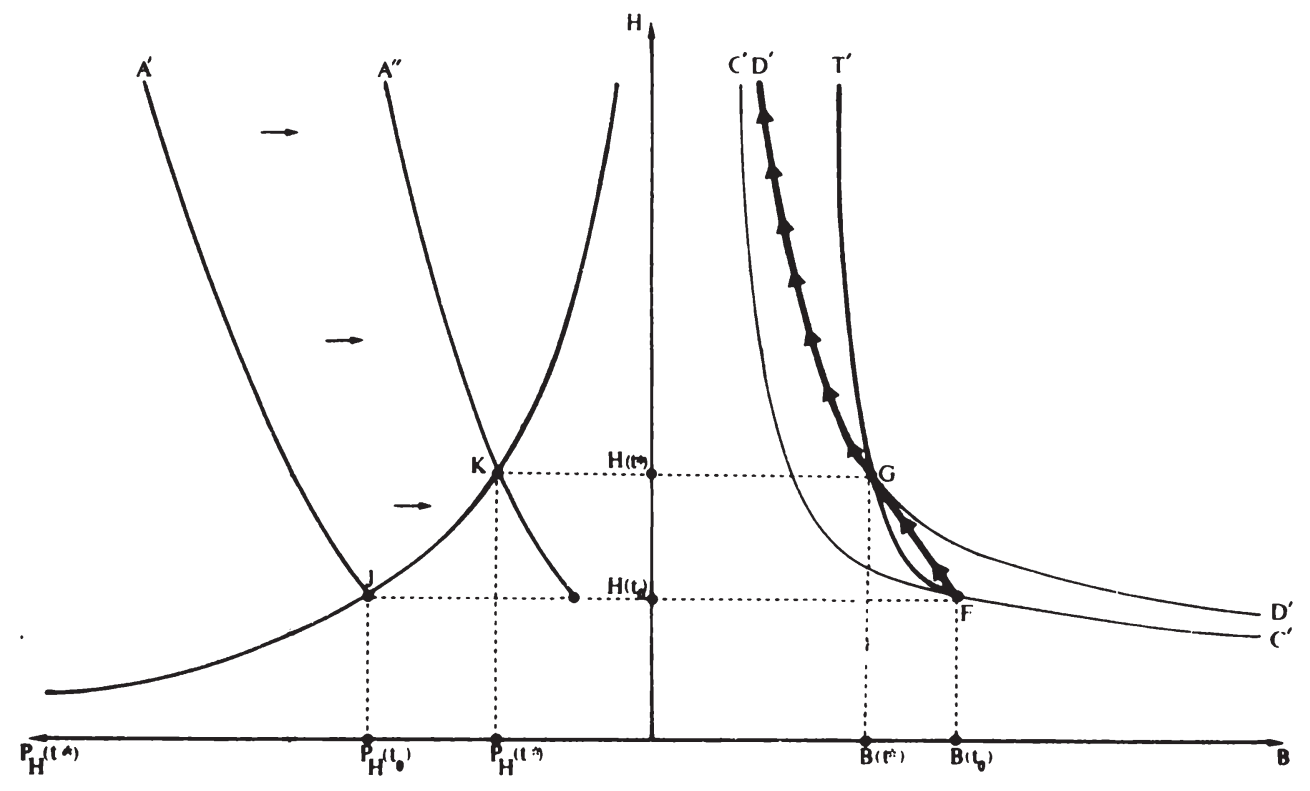

Punkt $J$ steht in Abb.10 für das Wohnungsmarktgleichgewicht, das unter dem Einfluß einer Grundsteuer zustandekommt, die sowohl den Boden- als auch den Gebäudewert trifft. Wird eine solche steuer durch eine nur den Bodenwert belastende steuer ersetzt, so verschiebt sich die Angebotskurve nach rechts in ihre neue Position A''. Lage und Steigung aller anderen Kurven ändern sich im AnschluB an die steuerreform nicht. Die intertemporalen Effekte dieses Wechsels in den Besteuerungsformen lassen sich am Verlauf des neuen Entwicklungspfades FGD' ablesen:

- Es wird im unmittelbaren AnschluB an die Steuerreform zu einem kurzfristigen Bauboom kommen - das system springt in realer zeit gerechnet von $F$ nach $G$, der in $t$ ovorhandene Wohnflächenbestand $H\left(t_{0}\right)$ wird im Verlauf der Kunstzeitphase $\left(t_{0}, t^{*}\right)$ an den neuen optimalen Bestand $H(t *)$ angepaßt. Die mit dieser Bestandsanpassung einhergehende Ausdehnung des Wohnleistungsan- 
gebots muß bei gegebener Nachfrage in $t * z u$ einem Sinken der Marktmieten führen. Entsprechend ist in Abb.10 das neue Gleichgewicht zum zeitpunkt $t *$ durch einen geringeren Häuserpreis $P_{H}(t *)$ gekennzeichnet. Darüberhinaus wird es in $t *$ zu einem Ansteigen der Baulandpreise kommen: die Bodermakler werden nur dann bereit sein, den für den steuerinduzierten Neubauboom erforderlichen Bauboden zur Verfügung zu stellen, wenn die Wohnungsinvestoren ihrerseits bereit sind, einen höheren Bodenpreis zu entrichten.

- Da die betrachtete Steuerreform die gleichgewichtigen Wachstumsraten unserer Modellökonomie aus Abschnitt III.2.1.1. unverändert läßt, kommt es auf lange sicht zu einer Anhebung des Zeitpfades für den Wohnflächenbestand; der Bestand an baureifen Grundstücken ist dagegen zu jedem Zeitpunkt jenseits von t* geringer, als er es gewesen wäre, wenn der Gesetzgeber auf die Reform des Grundsteuerrechts verzichtet hätte. Der Zeitpfad für den Häuserpreis und den Mietzins liegen dauerhaft unter, der des Bodenpreises ebenso dauerhaft über jenem, der sich unter dem Regime einer Grundsteuer geltenden Rechts eingespielt hat.

Angesichts dieser Resultate kommt man zu dem Schluß, daß die Bodenwertsteuer, ganz im Gegensatz zu RICARDOs eingangs zitierter These, keineswegs neutral ist. Der Grund für diese Diskrepanz ist in der Tatsache zu sehen, daB RICARDO die Bedeutung von Finanzinvestitionen als alternatives Medium der Vermögensbildung verständlicherweise nicht berücksichtigt hat. Da Kapitalmarktpapiere grundsteuerfrei sind und der Barwert der Grundsteuerzahlungen durch ein Ausweichen auf diese Aktiva reduziert werden kann, werden sich für die Grundvermögensbesitzer entsprechende Verhaltensweisen immer als lohnend erweisen. Hinzu kommt, daß die Bodenwertsteuer den Einsatz von Boden bei der Produktion von Wohnflächeneinheiten diskriminiert und es dem Wohnungsinvestor deshalb nahelegt, seine im Hinblick auf die Kapitalintensität der Bebauung in der steuerfreien welt getroffene Entscheidung $z u$ überdenken. 


\subsubsection{Eine allokationsneutrale Grundsteuer}

Die Grundsteuer in Form einer Bodenwertsteuer scheidet als Kandidat für eine neutrale Besteuerung des Grund und Bodens somit ebenso aus wie die Grundsteuer geltenden Rechts. Dies gilt auch für alle im politischen Raum zur Diskussion stehenden Reformvorschläge: zumindest bei den dem Verfasser bekannten Entwürfen handelt es sich um mehr oder weniger reinrassige Abkömmlinge der beiden bisher analysierten varianten.

Die Frage, die sich an dieser stelle zwangsläufig stellt, ist, ob es eine allokationsneutrale Besteuerung des Grund und Bodens überhaupt geben kann. Denn da alternative Vermögensbestände nicht mit Grundsteuer belastet werden, scheint es für die Bodeneigentümer doch immer attraktiv zu sein, ihr Portefeuille zugunsten dieser grundsteuerfreien Aktiva umzuschichten. Jedenfalls wurde dies in den vorangegangenen Abschnitten für substanzsteuern gezeigt, die unmittelbar am Bodenwert anknüpfen. Es liegt deshalb nahe, $z u$ untersuchen, wie eine steuer wirkt, bei der die Bodenfläche - ob bebaut oder nicht - Grundlage der Bemessung ist.

Im Entscheidungsproblem des Wohnungsinvestors würde diese Bodenflächensteuer die gesamte bebaute Bodenfläche $B *$ treffen, so daß man für die Grundsteuerzahllast des Wohnungsinvestors den folgenden Betrag erhielte:

(III.47)

$$
\mathrm{T}_{g r}=\tau_{g r} \mathrm{~B}^{*}
$$

$\mathrm{B}^{*}$ ist eine weitere Zustandsvariable im Entscheidungsproblem des Wohnungsinvestors. Infolge der Irreversibilität der Bebauung wird die zeitliche Entwicklung von $B^{*}$ durch die Bewegungsgleichung

$$
\left(\operatorname{III.48)} \quad \dot{\mathrm{B}} *=F^{\mathrm{d}}\right.
$$

beschrieben. 
Für das Planungsproblem des Wohnungsinvestors - es lautet

(III. 49)

$$
\{S, E, \varepsilon, F\} \quad M_{W}\left(t_{0}\right) \equiv \int_{t_{0}} A(t) \exp \left[-\operatorname{zr}\left(t-t^{*}\right)\right] d t
$$

unter den Bedingungen (II.2), (II.3), (II.9)', (II.10), (II.14), (II.16), (II.17a) und

(III.50)

$$
A=z\left(m H-E-r D-\tau_{g r} B^{*}\right)+S-F_{\varepsilon}^{d}-P_{B} F^{d}
$$

- 1 äßt sich die folgende Hamiltonfunktion formulieren:

(III.51)

$$
\begin{aligned}
H= & z\left(m H-E-r D-\tau_{g g^{B *}}\right)+s-F_{\varepsilon}{ }^{d}-P_{B} F^{d} \\
& +P_{H}\left[F^{d} \varphi(\varepsilon)-z(\alpha \delta H-E)\right] \\
& +\lambda_{D} S \\
& +\lambda_{B^{*}} F^{d} ;
\end{aligned}
$$

bei $\lambda_{B *}$ handelt es sich um den Schattenpreis des bebauten Bodens; es gilt

$$
\lambda_{B^{*}}=\frac{\partial M_{W}}{\partial B}=-\frac{\tau_{g r}}{r} ;
$$

$\lambda_{\mathrm{B}^{*}}$ signalisiert, um welchen Betrag der Marktwert des Wohnungsunternehmens sinkt, wenn - alles andere gleichbleibend - der Bestand der bebauten Bodenfläche um eine marginale Einheit angehoben wird; hinter $\lambda_{B^{*}}$ verbirgt sich der Barwert der auf diese marginale Einheit entfallenden Grundsteuerverpflichtungen.

Daß auch die Grundsteuer in Form der Bodenflächensteuer keine Auswirkungen hat auf die Zeitpfade der Nettoneuverschuldung, kann man durch die Berechnung der betreffenden optimalitätsbedingung leicht überprüfen. Auch die in Kapitel II bezüglich des Erhaltungsaufwandes angestellten Uberlegungen werden durch die Er- 
hebung einer solchen steuer nicht berührt (es gilt annahmegemäß $\left.P_{H}>1, y=1\right)$. Verzichten können wir auch auf die Differentiation von (III.51) nach der Zustandsvariablen B, die uns keine zusätzlichen Informationen zu liefern vermag.

Durch Differentiation von (III.51) nach $\varepsilon, F^{d}$ und $H$ erhalten wir als notwendige Bedingungen für den optimalen Investitionsplan des Wohnungsinvestors

(III.53)

$$
\mathrm{P}_{\mathrm{H}} \varphi^{\prime}(\varepsilon)=1 \text {, }
$$

$$
\frac{\beta}{\alpha} \varepsilon+\lambda_{B} *\left\{\begin{array}{l}
<\} \\
=j
\end{array} P_{B} \Rightarrow F^{d}\left\{\begin{array}{l}
= \\
\geq
\end{array}\right\} 0,\right.
$$

(III.55a)

$$
\hat{\mathrm{P}}_{\mathrm{H}}=0
$$$$
\text { für } t_{0} \leq t<t^{*} \text {, }
$$

(III.55b)

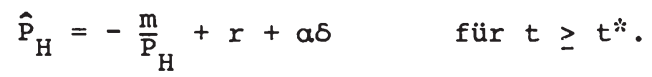

Nicht nur aus Gründen der analytischen Vereinfachung wollen wir mit dem modifizierten Bodenpreis $\overline{\mathbf{P}}_{B}$ weiterrechnen, wobei jedem Bodenpreis $\overline{\mathrm{P}}_{\mathrm{B}}$ durch die Beziehung

$$
\text { (III.56) } \quad \bar{P}_{B}=P_{B}+\frac{\tau_{g r}}{r}
$$

auf eineindeutige Weise ein Bodenpreis $\mathrm{P}_{\mathrm{B}}$ zugeordnet ist. Dementsprechend läBt sich Bedingung (III.54) auch schreiben als

$$
(\text { III.54) })^{\prime} \quad \frac{\beta}{\alpha} \varepsilon\left\{\begin{array}{l}
< \\
=
\end{array}\right\} \overline{\mathrm{P}}_{\mathrm{B}} \Rightarrow F^{\mathrm{d}}\left\{\begin{array}{l}
= \\
\geq
\end{array}\right\} 0 .
$$

Auch der Bodenmakler muß die Bodenflächensteuer in seinem Entscheidungskalkül berücksichtigen. Steuerlich belastet wird bei ihm der Bestand B an baureifen Grundstücken. Deshalb gilt es für den Bodenmakler, durch eine entsprechende Wahl des Zeitpfades furr das Baulandangebot das Optimierungsproblem

$$
\text { (III.57) } \quad \operatorname{Max}_{\left.F^{a}\right\}} M_{B}\left(t_{0}\right) \equiv \int_{t_{0}}^{\infty}\left[P_{B}(t) F^{a}(t)-z \tau g r^{B}(t)\right] \exp [-z r(t-t *)] d t
$$

unter den Bedingungen (II.1), (II.17a) und (II.45) zu Iösen. 
Entsprechend lautet die Hamiltonfunktion für diesen Planungskalkül

$$
H=P_{B} F^{a}-\tau_{g r} B-\lambda_{B} F^{a} \text {. }
$$

Durch partielle Differentiation von (III.58) nach der Kontrollvariablen $F^{\mathrm{a}}$ und nach der Zustandsvariablen $\mathrm{B}$ erhalten wir als notwendige Bedingungen für den nach steuer erlösmaximalen Angebotsplan für baureife Grundstücke im Falle einer inneren Lösung

$$
\begin{array}{lll}
\text { (III.59a) } & \hat{\mathrm{P}}_{B}=0 & \text { für } t_{0} \leq t<t *, \\
\text { (III.59b) } & \hat{\mathrm{P}}_{B}=r+\frac{\tau_{g r}}{r} & \text { für } t \geq t * .
\end{array}
$$

Unter Beachtung von (III.56) läßt sich (III.59b) umformen zu $(\text { III.59b })^{\prime} \quad \hat{\overline{\mathrm{P}}}_{\mathrm{B}}=r$.

Wie uns ein Blick auf die Bedingungen (III.53), (III.54)', (III.57a), (III.57b), (III.59a) und (III.59b)' bestätigt, ändern weder der Wohnungsinvestor noch der Bodenmakler unter dem Regime einer wertunabhängigen Bodenflächenflächensteuer ihre für die steuerfreie Welt abgeleiteten Verhaltensweisen: in keine der für unsere Analyse relevanten Optimalitätsbedingungen findet der Grundsteuersatz Eingang.

Eine Bodenflächensteuer der hier beschriebenen Art ist allokationsneutral.

Ein Blick auf die Definitionsgleichung (III.56) und die Bedingungen (III.54)' sowie (III.59b)' klärt uns auch darüber auf, warum dies so ist. Es gelingt dem Wohnungsinvestor, die Last der von ihm zu tragenden Grundsteuer in vollem Umfang auf den Bodenmakler $z u$ überwälzen. Bei im Vergleich zur Laisser-Faire Situation unveränderten Häuserpreisen und Wohnungsneubauplänen wechseln baureife Grundstücke ihre Eigentümer $z u$ einem Preis $\bar{P}_{B}$ je 
Flächeneinheit, der um den Barwert $\tau_{g r} / r$ aller aktuellen und zukünftigen Steuerverpflichtungen des Wohnungsinvestors unter dem Laisser-Faire-Bodenpreis $\mathrm{P}_{B}$ liegt. Aus diesem Grund könnte der Bodenmakler aus einer Änderung seiner erlösmaximierenden Laisser-Faire-Verkaufspläne keinerlei Vorteil ziehen: unabhängig davon, $z u$ welchem zeitpunkt er ein Grundstück zu verkaufen beabsichtigt, beträgt der Wert der von ihm für jede Flächeneinheit Bauland $\mathrm{zu}$ tragenden Grundsteuerlast $\tau_{\mathrm{gr}} / \mathrm{r}$.

So begrüßenswert die Implementierung einer Bodenflächensteuer unter wohlfahrtstheoretischen Gesichtspunkten also wäre, so sehr ist $z u$ befürchten, $d a \beta$ ein entsprechender Vorschlag im politischen Raum auf Skepsis stoßen würde. Auf den ersten Blick scheinen solche Vorbehalte auch berechtigt zu sein, läßt unsere Analyse doch den Eindruck entstehen, daß die Eigentümer wertvolleren Baulandes von einer allokationsneutralen Grundsteuer nicht stärker belastet werden dürfen als diejenigen geringerwertiger Grundstücke. Man übersieht hierbei aber, daB die Wahl eines einheitlichen, auf alle Grundstücke anzuwendenden Steuersatzes durch die Annahme eines homogenen Baulandbestandes notwendig wurde; hieraus zu schließen, diese wahl sei eine für das abgeleitete Neutralitätsergebnis notwendige Voraussetzung, wäre falsch; notwendig ist allein die Bedingung, daß sich eine allokationsneutrale Besteuerung des Grund und Bodens nicht unmittelbar am ökonomischen Wert des Grundstücks und dessen zeitlicher Entwicklung orientieren darf. Ist diese Bedingung nicht erfüllt, ist es dem Bodenmakler möglich, durch eine Vorverlegung des Verkaufszeitpunktes den Barwert der von ihm zu tragenden Grundsteuerlast $\mathrm{zu}$ verringern.

Nichts spricht aber dagegen, unterschiedlich wertvolle Grundstücke auch im Rahmen einer Bodenflächensteuer unterschiedlich zu belasten. Wie eine entsprechende Differenzlerung auch verwaltungstechnisch realisiert werden könnte, läßt sich dem "Entwurf für ein Gesetz zur Änderung des Grundsteuergesetzes und anderer Vorschriften" entnehmen, den der Hessische Minfster der Finanzen im Jahre 1971 vorgelegt hat. 54)

54) Hessischer Minister der Finanzen, zitiert in FRIAUF/RISSE/WINTERS(1978). 
Grundlage der in diesem Entwurf so bezeichneten "Flächengrundsteuer" ist die Elnordnung der Grundstücke in ein Wertstufensystem; die Einordnung selbst erfolgt nach der aktuellen Rangfolge der Bodenpreise. Bemessungsgrundlage der steuer ist der Anfangswert der jeweiligen Wertstufe. Hierauf wird ein einheitlicher, vom Gemeindeparlament $z u$ beschließender Vervielfältiger angewandt. Multipliziert man dieses Produkt mit der steuerpflichtigen Fläche des Grund und Bodens, so erhält man als Ergebnis den jährlichen Steuerbetrag. Wertermittlung, Aufstellung der Wertsstufenkarte und Festsetzung des Steuersatzes sollten nach Vorstellung des Hessischen Finanzministers jährlich erfolgen.

Ob die sogenannte "Flächengrundsteuer" tatsächlich allokationsneutral ist, hängt offensichtlich davon ab, wie die Anfangswerte der Wertstufen fortgeschrieben werden. Erfolgt diese Fortschreibung - wie im Gesetzesentwurf vorgesehen - entsprechend der aktuellen Bodenpreisentwicklung, so ist auch die Flächengrundsteuer ihrem ökonomischen Wesen nach eine Bodenwertsteuer und wie diese unter normativen Aspekten abzulehnen.

Wenn die Anfangswerte dagegen prinzipiell konstant bleiben was eine Anpassung in nicht vorherbestimmten längeren zeiträumen keineswegs ausschlieBt - und die jährliche Aufstellung der Wertstufenkarte lediglich mit einer Uberprüfung der Zuordnung der Grundstücke $z u$ den verschiedenen Wertstufen verbunden ist, ist die Flächengrundsteuer - die Konstanz der Vervielfältiger vorausgesetzt - allokationsneutral. 


\subsection{Die Vermögensteuer}

Das Grundvermögen zählt - ebenso wie das land- und forstwirtschaftliche Vermögen und die Grundstücke des Betriebsvermögens zum Gesamtvermögen des betreffenden Eigentümers ( $\$ 18$ BewG), der den Bestimmungen des Vermögensteuergesetzes (VStG) vom 17. April 1974 gemäß der Vermögensbesteuerung unterliegt. Die Vermögensteuer ist damit neben der Grundsteuer die zweite Substanzsteuer, die in die Entscheidungsprobleme von Wohnungsinvestoren und Bodenmaklern Eingang findet.

Die Bemessungsgrundlage für die steuer auf das Grundvermögen erhält man, wenn man vom Marktwert der Grundstücke das in diesen Grundstücken gebundene Fremakapital (und die sonstigen nach $\$ 118$ BewG abziehbaren Lasten) subtrahiert.

Innerhalb des verwendeten Modellrahmens gilt für das steuerpflichtige Vermögen $v_{W}$ des Wohnungsinvestors deshalb

$$
\mathrm{V}_{\mathrm{W}}=\mathrm{P}_{\mathrm{H}} \mathrm{H}-\mathrm{D} \text {. }
$$

Der Bodenmakler verfügt dagegen über ein steuerpflichtiges Vermögen in Höhe von

(III.61)

$$
\mathrm{V}_{\mathrm{B}}=\mathrm{P}_{\mathrm{B}} \mathrm{B}
$$

Neben den in den Definitionsgleichungen (III.60) und (III.61) beschriebenen Bemessungsgrundlagen gibt es noch eine weitere Größe, über die die Vermögensteuer auf die Planungen unserer Modellakteure Einfluß nimmt. Zu berücksichtigen ist nämlich auch, daß nicht nur Grundstücke, sondern grundsätzlich alle Vermögensformen von der Vermögensteuer erfaßt werden. So ist beispielsweise der Nettoertrag einer am Kapitalmarkt investierten D-Mark nicht mehr mit dem $z$ inssatz $r$ gleichzusetzen; abzuziehen von dieser Bruttorendite sind vielmehr noch die auf diese D-Mark entfallenden Vermögensteuerverpflichtungen in Höhe von ' ${ }^{v}$ ' wobei $\tau_{\mathrm{v}}$ den relevanten Vermögensteuersatz bezelchnet. Da wir in unserer Analyse als Diskontierungsfaktor die (Netto-) Rendite dieser 
alternativen Anlageform gewählt haben, müssen wir nun anstelle des bisher gültigen Diskontierungsfaktors $r$ den steuerverkürzten Satz

(III.62) $\quad r^{*}=r-\tau_{v}, \tau_{v}<r$

verwenden. Unter Berücksichtigung von (III.60), (III.61) und (III.62) lassen sich die Entscheidungsprobleme von Wohnungsinvestor und Bodenmakler deshalb wie folgt beschreiben.

Für den Wohnungsinvestor erhalten wir

$$
\{S, E, E, F\} M_{W}^{M} \equiv \int_{t_{0}}^{\infty} A(t) \exp \left[-z\left(r-\tau_{v}\right)\left(t-t^{*}\right)\right] d t
$$

unter den Bedingungen (II.2), (II.3), (II.9)', (II.10), (II.14), (II.16), (II.17a) und

$$
A=z\left[m H-E-r D-\tau_{v}\left(P_{H} H-D\right)\right]+s-F^{d} \varepsilon-P_{B} F^{d} .
$$

Der Bodenmakler sieht sich dagegen dem folgenden Entscheidungsproblem gegenüber:

$$
\operatorname{Max}_{\left\{F^{a}\right\}} \quad M_{B} \equiv \int_{t_{0}}^{\infty}\left(P_{B} F^{a}-z \tau_{v} P_{B} B\right) \exp \left[-z\left(r-\tau_{v}\right)\left(t-t^{*}\right)\right] d t
$$

unter den Bedingungen (II.1), (II.17a) und (II.45).

Bildet man die zu (III.63) und (III.65) korrespondierenden Hamiltonfunktionen und differenziert diese partiell nach den entsprechenden Kontroll- und Zustandsvariablen, so errechnet man als notwendige Bedingungen für die sich nach der Erhebung einer Vermögensteuer der hier beschrlebenen Art einstellenden Wohnungs- und Baubodenmarktgleichgewichte

(III.66) FF $\sim \mathrm{SF}$, 


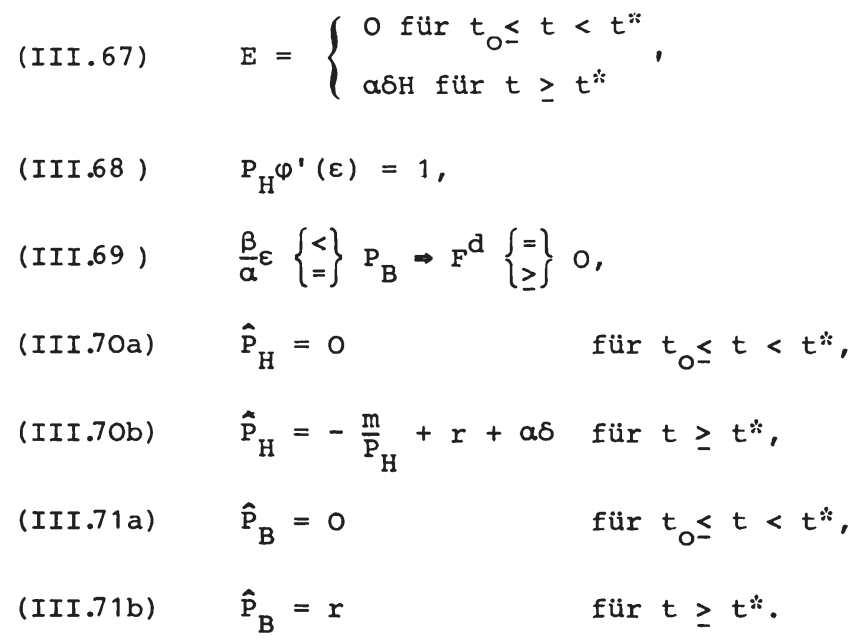

Wirft man einen Blick auf die entsprechenden Bedingungen für die paretoeffiziente Laisser-Faire-Allokation und vergleicht diese mit den Bedingungen (III.66) bis (III.71b), so wird man unschwer feststellen können, daß sich erstere unter dem Einfluß der Vermögensteuer nicht verändert haben:

Die Vermögensteuer ist - zumindest dann, wenn sie in der hier beschriebenen Art und weise erhoben wird - allokationsneutral.

Dieses Ergebnis mag auf den ersten Blick überraschen. Insbesondere ist man geneigt anzunehmen, daß es für den Wohnungsinvestor unter dem Regime einer allgemeinen Vermögensteuer günstiger ist, mit Fremdkapital zu finanzieren, da die Bemessungsgrundlage der Vermögensteuer offensichtlich umso geringer ist. Der Grund, warum weder die Selbst- noch die Fremdfinanzierung bevorzugt werden, ist in der Tatsache $z u$ sehen, daB der Wohnungsinvestor sich bei der Wahl der optimalen Finanzierungsform ausschlieblich an den Interessen der Unternehmenseigner orientiert. Und für diese spielt die Art der Finanzierung keine Rolle: sie müssen Vermögensteuer sowohl für das in ihrem Unternehmen gebundene Eigenkapital als auch für Unternehmensobligationen zahlen, die in unserem Modell neben bebauten und unbebauten Grundstücken das dritte Vermögensanlagemedium darstellen. 
Aus dem gleichen Grund ändern sich unter dem Einfluß der Vermögensteuer weder die paretoeffizienten Investitionsentscheldungen des Wohnungsunternehmers, noch der paretoeffiziente Bodenverkaufsplan des Bodenmaklers. Beide erkennen, daß der repräsentative Haushalt - dessen Interessen sie vertreten Vermögensteuer sowohl auf die Werte der Häuser- und Baulandbestände als auch auf jede andere Vermögensform zahlen müßte, die er mit dem durch einen Verzicht auf Wohnungsinvestitionen bzw. durch einen vorgezogenen Verkauf baureifer Grundstücke freiwerdenden Eigenkapital erwerben könnte; der Barwert der Vermögensteuerzahllast würde sich aus der sicht des einzelnen Eignerhaushalts infolge dieser Vermögensumschichtung nicht verändern.

Die Gültigkeit dieses Neutralitätsergebnisses ist allerdings an Restriktionen geknüpft, die im Rahmen des gegenwärtig praktizierten Verfahrens der Vermögensbesteuerung nicht alle erfüllt sind.

Als erste Ursache für eine denkbare Nichtneutralität des geltenden Vermögensteuerrechts ist die vermögensteuerliche Ungleichbehandlung natürlicher und juristischer Personen $\mathrm{zu}$ nennen: zur Vermögensteuer herangezogen werden nämlich nicht nur diejenigen natürlichen Personen, die die Bedrngungen des $\S 1$, Absatz 1 und 2 vStG erfüllen, sondern auch juristische Personen, soweit sie Sitz und Geschäftsleitung im Inland haben. Da sich Unternehmen mit eigener Rechtsperson ihrerseits aber wiederum im Eigentum natürlicher Personen befinden und deren Unternehmensbeteiligungen in Form von Einlagen oder Aktien ebenfalls zum steuerpflichtigen Gesamtvermögen zählen, kommt es zu einer Doppelbelastung des Eigenkapitals von Körperschaften. Dieser Doppelbelastung kann das körperschaftliche Wohnungsunternehmen offensichtlich nur durch ein Ausweichen auf die Fremdfinanzierung entgehen. Nur wenn dem Unternehmen dieses Ausweichen vollständig gelingt - ein in der Praxis wohl kaum vorzufindender Grenzfall - erwachsen dem körperschaftlichen Wohnungsunternehmen und dem Wohnungsunternehmen in der Rechtsform einer Personengesellschaft Finanzierungskosten gleicher Höhe. Man wird 
deshalb erwarten können, daß in diesem Grenzfall auch beider Investitionsentscheidungen dieselben sind.

Ist die vollständige Finanzierung mit Fremdkapital dagegen nicht möglich, wird die steuerliche Diskriminierung juristischer Personen auch Auswirkungen auf deren Investitionsverhalten haben: eine von ihnen vorgenommene Unternehmensexpansion ist, vergleicht man sie mit den Expansionskosten von Personengesellschaften, infolge der vermögensteuerlichen Doppelbelastung des Unternehmenskapitals teurer.

Verschärft werden diese Belastungsunterschiede durch die einschlägigen Vorschriften der $\S \S 6$ und 8 vStG. Durch die in §6 VStG genannten Freibeträge wird die aligemeine Vermögensteuerpflicht natürlicher Personen aufgeweicht. Für jede in die Vermögensteuerveranlagung einbezogene Person wird ein Freibetrag in Höhe von 70000 D-Mark gewährt. Da das Gesetz darüberhinaus die Möglichkeit einer Zusammenveranlagung mit den zum Haushalt gehörenden Kindern einräumt ( $\$ 14$, Absatz 2 VStG), haben diese Freibeträge zur Folge, daß bei einer Durchschnittsfamilie, die neben ihrem Eigenheim kein erhebliches Vermögen mehr besitzt, im Regelfall keine Vermögensteuer anfällt. Im Gegensatz zu natürlichen Personen wird juristischen Personen im Rahmen der Vermögensteuer kein Freibetrag eingeräumt, für sie ist die Freigrenze des §8, Absatz 1 VStG relevant: eine Vermögensteuer wird bei juristischen Personen nur dann erhoben, wenn deren Gesamtvermögen mindestens 20000 D-Mark beträgt.5)

Hinzu kommt, daß juristischen Personen im Vergleich zu natürlichen ein höherer Vermögensteuersatz auferlegt wird $(0,7$ Prozent im Vergleich zu 0,5 Prozent).

Ein zweiter Grund, der zur Relativierung der Neutralitätsaussage zwingt, sind die im Rahmen der Grundsteueranalyse beschriebenen Besonderheiten der praktizierten Bewertungsver-

55) Es sei an dieser Stelle auch auf die besonderen Vemögensteuerbefreiungen des $\S 7$ VStG hingewiesen. 
fahren. Grundstücke sind nämlich auch bei der Bestimmung des steuerpflıchtigen Vermögens mit ihrem Einheitswert anzusetzen (§114, Absatz 3 BewG). Und hierbel kommt es nun $z u$ denselben Bewertungsmängeln, die wir in Abschnitt III.2.1.1. mit Hilfe des Parameters $\gamma_{1}$ beschrieben haben. Aufgrund der Begünstigung bebauter Grundstücke bzw. der bewertungsrechtlichen Diskriminierung baureifer wird es für den vermögenbildenden und -anlegenden Haushalt attraktiver, im AnschluB an die Einführung einer Vermögensteuer geltenden Rechts Vermögen in stärkerem MaBe als in der steuerfreien Welt in Form von Wohnimmobilien zu bilden - der Wohnungsinvestor wird deshalb unter sonst gleichen Bedingungen versuchen, in $t_{0}$ (dem zeitpunkt der erstmaligen Erhebung der Steuer) seinen Bestand an Wohnflächeneinheiten auszudehnen.

Graphisch lassen sich die aus dieser steuerinduzierten Verhaltensänderung des Wohnungsinvestors resultierenden intertemporalen Verzerrungen ableiten, wenn man bedenkt, daß Bedingung (III.7Ob) für $\gamma_{1} \neq 1 \mathrm{zu}$

$$
(\text { III.7Ob })^{\prime} \quad \hat{\mathrm{P}}_{\mathrm{H}}=-\frac{\mathrm{m}}{\mathrm{p}_{\mathrm{H}}}+r+\alpha \delta+\left(1-\gamma_{1}\right) \tau_{\mathrm{V}}
$$

wird. Indem man (III.7Ob)' nach $\mathrm{P}_{\mathrm{H}}$ auflöst, erhält man die Gleichung für die Nachfragekurve $\mathrm{N}^{\prime}$ in $\mathrm{Abb} .11$ :

$$
P_{H}=\frac{m(a / H)}{(1-\beta) r+\alpha \delta-\left(1-\gamma_{1}\right) \tau_{v}} .
$$

Für $\gamma_{1} \neq 1$ ist bei gegebenem Häuserbestand $H$ der Häuserpreis $\mathrm{P}_{\mathrm{H}}$ höher als im Falle der bewertungsrechtlichen Gleichbehandlung bebauter und baureifer Grundstücke. Deshalb liegt die Nachfragekurve $N^{\prime}$ links der Laisser-Faire-Nachfragekurve $N$.

Hätte sich das System in der steuerfreien welt und ebenso bei der wahl der oben beschriebenen idealtypischen Vermögensteuer entlang der isoelastischen Kurve FC bewegt, so ließe sich der EinfluB der Diskriminierung baureifer Grundstücke im Rahmen der Grundstücksbewertung auf die Modelldynamik am Verlauf des neuen 
$\mathrm{Abb} \cdot 11$

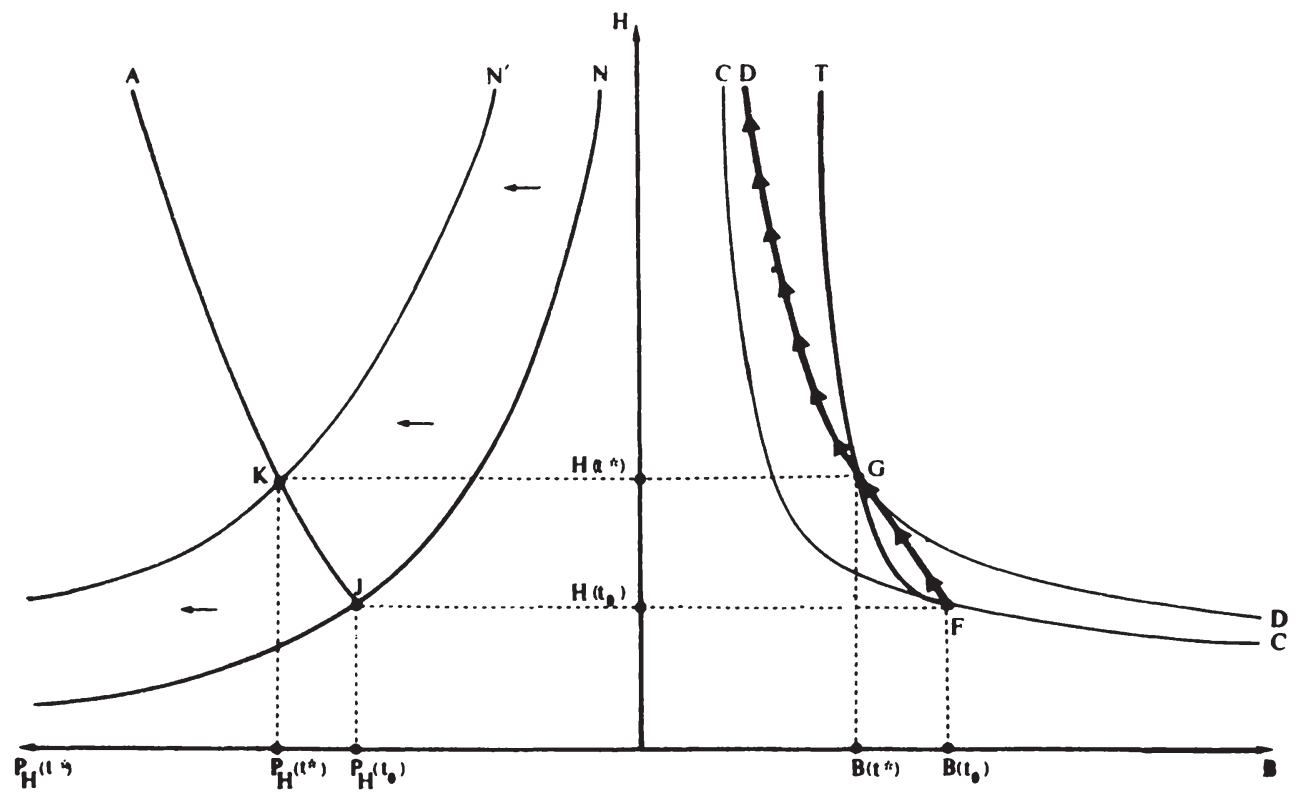

Entwicklungspfades FGD ablesen:

Nach Einführung einer Vermögensteuer, die der hierzulande erhobenen entspricht, kommt es zu einem kurzfristigen Neubauboom - das system springt in realer zeit von F nach $G$. Im Zuge dieses Neubaubooms steigt der Bodenpreis, das Niveau der wohnungsmieten und das des Häuserpreises sinken.

Nachdem diese Anpassungsreaktion abgeebbt ist, entwickelt sich die Modelibkonomie entlang dem Pfad GD weiter, wobei dies geht aus den Bedingungen (III.68), (III.69), (III.7Ob)' und (III.71b) hervor - sich die Dynamik der modellendogenen Varlablen mit Hilfe der für die Laisser-Faire-ðkonomie charakteristischen Wachstumsraten beschreiben läBt: Auf lange sicht kommt es deshalb zu einer dauerhaften Anhebung des zeitpfades für den wohnflächenbestand. Aufgrund der steuerbedingt gestiegenen Rentabilität des Wohnimmobilienvermögens ist es für den wohnungsinvestor lohnend, zu jedem Zeitpunkt jen- 
seits von t* einen im Vergleich zur Laisser-Faire-Situation gröBeren Bestand an wohnimmobilien zu halten. Auch der zeitpfad des Bodenpreises wird infolge der unveränderten wachstumsrate $\hat{\mathrm{P}}_{\mathrm{B}}$ dauerhaft über seinem Laisser-Faire-Niveau liegen. Dem mit der dauerhaften Bodenpreissteigerung verbundenen Anstieg der Neubaukosten weicht der wohnungsinvestor - jedenfalls zum Teil - dadurch aus, daß en zu jedem zeitpunkt jenseits von $t^{*}$ eine im Vergleich zur steuerfreien welt höhere Kapitalintensität der Bebauung wählt. Dauerhaft absinken werden dagegen die zeitpfade für den Mietzins und den Häuserpreis. Auch der Bestand an baureifen Grundstücken wird zu jedem Zeitpunkt nach Einführung der steuer geringer sein, als er es bei einem Verzicht auf die Vermögensbesteuerung bzw. die Vermeidung der ihr zugrundeliegenden Bewertungsmängel gewesen wäre.

Zwei weitere Gründe, die Zweifel an der zuvor abgeleiteten Neutralität der vermögensteuer laut werden lassen (und die mitverantwortlich dafür sind, daß wir unseren normativen wertungen einzelner steuerarten das second-best-Kriterium zugrundelegen), lassen sich im Rahmen unseres partialanalytischen Ansatzes dagegen nicht greifen:

- zum einen ist dies die Tatsache, daß nur die bewertbaren vermögensbestände des Haushalts durch die steuer erfaBt werden. Das Humankapital des Haushalts, das sich einer objektiven Bewertung entzieht, bleibt vermögensteuerfrei. Es steht zu erwarten, daß sich die Vermögensstruktur des Haushalts zugunsten dieses steuerlich präferierten Bestandes verschiebt; eine Ausdehnung der Ausbildungszeit zulasten des Arbeitszeitangebots wird attraktiv;

- zum anderen sind dies die Einflüsse, die die Vermögensteuer auf die Konsum-Spar-Entscheidung der privaten Haushalte ausübt. Im Paretooptimum der Laisser-Faire-ర̌konomie entspricht die Zeitpräferenzrate der Haushalte der. Rendite des im wohnungssektor gebundenen Vermögens, die threrseits wiederum Bedingung für ein Gleichgewicht - übereinstimmen muB mit dem 
Kapitalmarktzins $r$ : die Haushalte bestimmen ihren Konsumplan unter Zugrundelegung ihrer Lebenszeiteinkommen in der Weise, daB Im Gleichgewicht der aus einer zeitlichen Vorverlegung von Konsum resultierende Grenznutzen (der mit Hilfe der zeitpräferenzrate gemessen wird) übereinstimmt mit den durch das damit einhergehende Entsparen entstandenen Kosten bezogen auf die letzte verausgabte $D$-Mark sind dies Kosten in Form entgangener Kapitalmarkterträge bzw. zu zahlender Kreditzinsen, jewells in Höhe von $r^{56}$ ) Unter dem Einfluß der Vermögensteuer verringern sich für den Haushalt nun aber diese mit dem Entsparen verbundenen Kosten - die Zeitpräferenzrate des Haushalts wird nach Steuer mit dem steuerverkúrzten Nettozinssatz $r^{*}=r-\tau_{v}$ ubereinstimmen. Dagegen ändert sich unter dem ElnfluB der Vermögensteuer die Bedingung für das Portefeuillegleichgewicht nicht: die Rendite $\left(m / \mathrm{P}_{\mathrm{H}}+\hat{\mathrm{P}}_{\mathrm{H}}-\infty\right)$ des Wohnimmobilienvermögens stimmt nach wie vor überein mit dem Bruttorealzins $\mathrm{r}$ [vgl. Bedingung (III.7Ob)]. Die Vermögensteuer treibt auf diese Weise einen Keil zwischen die Zeitpräferenzrate der Haushalte und die Rentabilität des Anlagevermögens. Indem sie für die privaten Haushalte das Entsparen attraktiver macht und so die gesamtwirtschaftliche Kapitalbildung diskriminiert, verletzt sie die Bedingung für ein intertemporales Paretooptimum.

Wir wollen uns in den nun folgenden Kapiteln von der Bestandsbesteuerung abwenden und unsere weiteren tberlegungen der Besteuerung von Leistungsströmen widmen, die innerhalb und zwischen den betrachteten Sektoren fließen. Ansatzpunkte für solche Steuern bieten

- die aus dem Verkauf von Bauland fließenden Erlöse,

- die Ausgaben fur den Konsum von Wohnleistungseinheiten sowie

- die Einkünfte bzw. Gewinne, die im zuge der Nutzung von Wohnimmobilien erztelt werden.

56) Vgl. auch Abschnitt I.3 dieser Arbeit 
3. Die Besteuerung des Bodenverkehrs - ist die Grunderwerusteuer tatsächlich eine Mobilitätsbremse?

Im Rahmen des bundesdeutschen Steuersystems wird nicht nur das Eigentum an Grund und Boden mit Steuern belegt, auch der (entgeltliche) Eigentumsübergang wird durch eine spezielle steuer, die Grunderwerbsteuer, belastet. Das Bemerkenswerte an der Grunderwerbsteuer - wie im ubrigen an allen Verkehrsteuern, zu denen sie steuersystematisch zählt - ist, daß ihr alleiniger Zweck darin besteht, dem offentlichen Sektor Einnahmen zu verschaffen.

Diese rein fiskalische Zwecksetzung erzwingt es geradezu, die Neutralität der Grunderwerbsbesteuerung $z u$ fordern. Aber gerade diese Eigenschaft scheint der Steuer, schenkt man der herrschenden Literaturmeinung Glauben, zu fehlen. Ihr zufolge verteuern und erschweren Verkehrsteuern im allgemeinen und die Grunderwerbsteuer im speziellen die von ihnnen erfaßten Transaktionen; insbesondere erschwere die Grunderwerbsteuer auch die optimale Allokation des Bodens, die "Wanderung des Bodens zum besten Wirt." 57 )

Interessant wird es nun sein zu untersuchen, wie diese Steuer in unserem Modell auf die Planungen von Wohnungsinvestoren und Bodenmaklern einwirkt ${ }^{58}$ ) Denn di.e oben zitierte These wird im allgemeinen vor dem Hintergrund im Zeitablauf konstanter Bodenpreise formuliert.

Bemessungsgrundlage der Grunderwerbsteuer ist der "Wert der Gegenleistung", d.h. der Kaufpreis (samt Nebenkosten). Ist $\tau_{\text {gre }}$ der Grunderwerbsteuersatz, so beträgt der vom Wohnungsinvestor zu zahlende Bruttobodenpreis $\mathrm{P}_{\mathrm{B}}^{\mathrm{br}}$

$$
\mathrm{P}_{\mathrm{b}}^{\mathrm{br}}=\left(1+\tau_{g r e}\right) \mathrm{P}_{\mathrm{B}}{ }^{59)}
$$

57) Vgl. 2.B. PAHLKE(1980), S.263.

58) Vgl. auch $\operatorname{SINN}(1984)$.

59) Geschuldet wind die Grunderwerbsteuer van Kaufer und van Verkalufer zur gesamten Hand. 
Unter Berücksichtigung von (III.73) und der Annahme $\mathrm{P}_{\mathrm{H}}>1$ (woraus wiederum $y=1$ folgt) lassen sich die Planungsprobleme des repräsentativen Wohnungsinvestors und des repräsentativen Bodenmaklers dann wie folgt formulieren.

(III. 74) $\{S, E, \varepsilon, F\} \quad M_{W} \equiv \int_{t_{0}}^{\infty} A(t) \exp [-z r(t-t *)] d t$ unter den Bedingungen (II.2), (II.3), (II.9)', (II.10), (II.14), (II.16), (II.17a) und

$$
A=z(m H-E-r D)+S+F_{\varepsilon}^{d}-P_{B}\left(1+\tau_{g r e}\right) F^{d},
$$

bzw.

(III. 76)

$$
\left\{F^{a}\right\}
$$

$$
M_{B} \equiv \int_{t_{0}}^{\infty} P_{B}(t)\left(1+\tau_{g r e}\right) F^{a}(t) \exp [-z r(t-t *)] d t
$$

unter den Bedingungen (II.1), (II.17a) und (II.45).

Bildet man die zu den Planungsproblemen (III.74) und (III.76) gehörenden Hamiltonfunktionen und differenziert diese nach den entsprechenden Kontroll- und Zustandsvariablen, so erhält man neben der Aussage, daß auch die Grunderwerbsteuer die optimalen Finanzierungs- und Werterhaltungspläne der Laisser-Faire-ökonomie nicht tangiert, die folgenden für ein Gleichgewicht notwendigen Bedingungen:

$$
\begin{aligned}
& \text { (III. 7T) } \quad \mathrm{P}_{\mathrm{H}^{\varphi^{\prime}}}(\varepsilon)=1 \text {, } \\
& \text { (III. 78) } \quad \frac{\beta}{\alpha} \varepsilon\left\{\begin{array}{l}
\alpha \\
=
\end{array}\right\} P_{B}\left(1+\tau_{g r e}\right) \Rightarrow F^{d}\left\{\begin{array}{l}
= \\
\geq
\end{array}\right\} 0 \\
& \text { (III. 79a) } \hat{\mathrm{P}}_{\mathrm{H}}=0 \text { für } t_{\mathrm{O}} \leq t<t \text {, } \\
& \text { (III. 79b) } \hat{\mathrm{P}}_{\mathrm{H}}=-\frac{\mathrm{m}}{\overline{\mathrm{P}}_{\mathrm{H}}}+r+a \delta \text { für } t \geq t \text {, } \\
& \text { (III. 80a) } \hat{\mathrm{P}}_{\mathrm{B}}=0 \quad \text { für } t_{0} \leq t<t \text {, } \\
& \text { (III. 80b) } \quad \hat{\mathrm{P}}_{B}=r \quad \text { für } t \geq t \text {. }
\end{aligned}
$$


Vergleicht man diesen Katalog von Bedingungen mit jenem, den wir für eine steuerfrei Welt abgeleitet haben, so erkennt man, daß die Grunderwerbsteuer keinen EinfluB ausübt auf den Anfangszustand und die zeitliche Entwicklung unserer Modellökonomie: Weder ändern die Wohnungsinvestoren ihre in der steuerfreien Welt getroffenen Investitionsentscheidungen, noch revidieren die Bodenmakler ihren ursprünglichen Angebotsplan für baureife Grundstücke.

Die Grunderwerbsteuer ist allokationsneutral.

Die Ursache dieser Neutralität ist die Tatsache, daß die Steuer "immer nur den Verkäufer trifft", auch dann, wenn die Zahllast beim Käufer liegt. Dies bringt Bedingung (III.78) zum Ausdruck: bei gegebenem Wohnungsneubauplan des Wohnungsinvestors ist auch der Zeitpfad des Bodenwertgrenzproduktes festgelegt und mit ihm der Zeitpfad des Bruttobodenpreises $P_{B}\left(1+\tau_{g r e}\right)$; einen höheren als diesen Preis wird der Wohnungsinvestor für eine Flächeneinheit Bauland in keinem Fall zu zahlen bereit sein. Da der Zeitpfad des Bruttobodenpreises somit mit dem Bodenpreispfad der Laisser-Faire-Ökonomie identisch ist, mus der Pfad des Nettopreises nach Maßgabe des Grundsteuersatzes $\tau_{\text {gre }}$ sinken.

Es liegt der Einwand nahe, daß der Bodenmakler auf diese Uberwălzung sehr wahrscheinlich mit einer Veränderung seines Baulandangebots reagieren wird. Okonomisch rationel wäre eine solche Verhaltensweise aber nur dann, wenn sich dadurch der Barwert der steuerzahlungen verringern liebe. Genau dies ist aber nicht möglich. Da der Bodenpreis und mit ihm die Bemessungsgrundlage der Grunderwerbsteuer von Periode zu Perlode mit einer Rate in Höhe des Marktzinses und Diskontierungsfaktors $r$ wächst, ist der steuerbarwert zum Betrachtungsze1tpunkt $t_{0}$ aus der sicht des einzelnen Bodenmaklers immer derselbe, unabhängig davon, $\mathrm{zu}$ welchem zeitpunkt das betreffende Grundstück verkauft wird. Dle erlösmaximierende strategie des Bodenmaklers wird es deshalb sein, den für die steuerfreie Welt aufgestellten 
Bodenangebotsplan aufrechtzuerhalten und die Last der Grunderwerbsteuer zu tragen.6)

Bei der tbertragung dieser Ergebnisse auf die aktuelle Situation in der steuerlichen Behandlung von Grundstücksveräußerungen ist allerdings aus zwei Gründen Vorsicht geboten:

- zum einen läßt sich die Zahlung der Grunderwerbsteuer umgehen, wenn die Funktionen des Bodeneigentümers und des Wohnungsinvestors in einer Person vereinigt sind. In diesem Falle findet im Zuge der Bebauung kein Eigentumsübergang statt - und nur ein solcher wird von der Grunderwerbsteuer erfaßt -, sondern lediglich eine Nutzungsänderung des Grundstücks. Diese Möglichkeit der "Steuervermeidung" im Rahmen des geltenden Grunderwerbsteuerrechts hat zwar keine unmittelbaren allokativen Folgen, sie stimmt aber unter verteilungspolitischen Aspekten und im Hinblick auf den dem gesamten steuerrecht zugrundeliegenden Gleichbehandlungsgrundsatz bedenklich;

- zum anderen erfaßt die Grunderwerbsteuer nicht nur - wie in der Analyse implizit unterstellt - den Wechsel in der Bodennutzung, sondern jeden entgeltlichen Eigentumsübertrag in Form eines Rechtsgeschäfts. Da die Höhe der steuerlichen Belastung somit von der Häufigkeit der Eigentumsübergänge abhängig ist, erweist sich die These von der mobilitätshemmenden Wirkung des geltenden Grunderwerbsteuerrechts ${ }^{61)}$ zumindest dann als stichhaltig, wenn Wohnungsinvestoren oder Bodenmakler planen, ihre Immobilienbestände innerhalb des Planungshorizonts $\left(t_{0}, \infty\right)$ mehr als einmal umzuschichten.

60) Die einzige Möglichkeit, der Grunderwerbsteuerbelastung zu entgehen, bestünde für den Bodenmakler darin, auf den Verkauf seines Grundstüicks ganz zu verzichten. Allerdinqs käme er dann nicht in den Genuß des Verkaufserlöses; er könnte auch niemals die eingetretenen Wertsteigerungen seines Grundstücks realisieren. Bei einem Steuersatz von weniger als hundert Prozent wäre dies sicherlich nicht die gewinmaximale Strategie.

61) ANDREAE (1980), S.595. 
Nun ist unsere Analyse aber insofern hilfreich, als sie uns AufschluB darüber gibt, wie eine "Grunderwerbsteuer" ausgestaltet werden mübte, wollte sie einer unter Effizienzgesichtspunkten durchgeführten Beurteilung standhalten:

Eine solche Steuer darf offensichtlich nicht an Markttransaktionen anknüpfen, sondern muß sich an Änderungen in der Bodennutzung orientieren - auch dann, wenn mit dem Nutzungswechsel kein Eigentumswechsel einhergeht. 62)

Auf diese Weise wäre garantiert, daß weder Wohnungsinvestorer, die über eigenen Grundbesitz verfügen, steuerlich bevorzugt behandelt werden, noch die Last der Grunderwerbsteuer mit der Anzahl der Transaktionen innerhalb der verschiedenen Bestände wächst.

62) Eine solche Steuer hat in die englischsprachigen Literatur unter der Bezeichnung "Severance Tax" Eingang gefunden;- vgl. z.B. PAGE(1977), Kapitel 6, BESSERER(1984). 
4. Die Besteuerung der Mietumsätze

4.1. Die generelle umsatzsteuerbeferiung

Der Umsatzsteuer unterworfen sind alle

- entgeltlichen Lieferungen und

- sonstigen Leistungen sowie

- Eigenverbräuche,

soweit sie durch ein Unternehmen im Erhebungsbereich durchgeführt werden ( $\$ 1$, Absatz 1 UStG).

Obwohl jede dieser drei Umsatzarten auch im Zusammenhang m1t Wohnungseigentum möglich ist, hat der Gesetzgeber verfügt, daß die aus der wirtschaftlichen verwertung von wohngrundstücken fließenden Umsätze grundsätzlich umsatzsteuerfrei sind (§4, Ziffer $12 \mathrm{a}$ UStG). Diese Steuerbefreiung hat zur Konsequenz, da $B$ die mit den Umsätzen aus Vermietung und Verpachtung verbundenen, im Zuge der Errichtung und der Werterhaltung des (vermieteten oder selbstgenutzten) Gebäiudes gezahlten Mehrwertsteuerbeträge vom Finanzamt nicht zurückerstattet werden ( $\$ 15$, Absatz 2 UStG).

Um die allokativen Auswirkungen dieser generellen Umsatzsteuerbefreiung des Wohnungssektors aufzeigen zu können, empfiehlt es sich, zunächst eine ökonomie zu betrachten, in der auch die im Wohnungssektor anfallenden Umsätze der Umsatzbesteuerung unterliegen und die beim Kauf von Bauleistungen in Rechnung gestellte Mehrwertsteuer im Rahmen des Vorsteuerabzugs zurückgezahlt wird. Der Verkauf von Bauland sei dagegen - wie dies in der realen welt tatsächlich auch der Fall ist - umsatzsteuerfrei.

Um die Allokationseffizienz einer allgemeinen Umsatzsteuer korrekt beurteilen zu können, bedarf es keines aufwendigen mathematischen optimierungskalküls. Es erübrigt sich nämlich, den Mehrwertsteuersatz $\tau_{u}$ explizit in unserer Analyse der Mehrwertsteuerinzidenz zu berücksichtigen; vielmehr lassen sich die in den Laisser-Faire-Optimalitätsbedingungen

$$
E=\left\{\begin{array}{l}
0 \text { für } t_{0} \leq t<t^{*} \\
\alpha \delta H \text { für } t \geq t^{*}
\end{array},\right.
$$


(II.55)

$$
\begin{aligned}
& P_{H} \varphi^{\prime}(\varepsilon)=1, \\
& \frac{B}{\alpha} \varepsilon\left\{\begin{array} { l } 
{ < \} } \\
{ = j }
\end{array} P _ { B } \Rightarrow F ^ { d } \left\{\begin{array}{l}
j=\} \\
\geq j
\end{array},\right.\right.
\end{aligned}
$$

$$
\hat{\mathrm{P}}_{\mathrm{H}}=-\frac{\mathrm{m}}{\mathrm{P}}_{\mathrm{H}}+r+\alpha \delta \quad \text { für } t \geq t * \text {, }
$$

$$
\hat{\mathrm{P}}_{\mathrm{H}}=\hat{\mathrm{P}}_{\mathrm{B}}=0
$$

für $t_{0} \leq t<t *$,

$$
\hat{\mathrm{P}}_{\mathrm{B}}=\mathrm{r}
$$

für $t \geq t *$

enthaltenen Preise und deren Wachstumsraten auch als Nettogrößen (nach Abzug der anteiligen Mehrwertsteuer) interpretieren. Für die betreffenden Bruttopreise und deren Wachstumstumsraten muß dann entsprechend gelten:

$$
\begin{array}{ll}
\text { (III.8 1) } & \mathrm{m}^{\mathrm{b}}=\left(1+\tau_{\mathrm{u}}\right) \mathrm{m}, \hat{\mathrm{m}}^{\mathrm{b}}=\hat{\mathrm{m}}, \\
\text { (III.8 2) } & \mathrm{P}_{\mathrm{H}}^{\mathrm{b}}=\left(1+\tau_{\mathrm{u}}\right) \mathrm{P}_{\mathrm{H}^{\prime}}, \hat{\mathrm{P}}_{\mathrm{H}}^{\mathrm{b}}=\hat{\mathrm{P}}_{\mathrm{H}^{\prime}} \\
(\text { III.8 3) } & \mathrm{P}_{\mathrm{B}}^{\mathrm{b}}=\left(1+\tau_{\mathrm{u}}\right) \mathrm{P}_{\mathrm{B}^{\prime}}, \hat{\mathrm{P}}_{\mathrm{B}}^{\mathrm{b}}=\hat{\mathrm{P}}_{\mathrm{B}} \\
\text { (III.8 4) } & \mathrm{P}_{I}^{\mathrm{b}}=1+\tau_{\mathrm{u}}, \hat{\mathrm{P}}_{I}^{\mathrm{b}}=\hat{\mathrm{P}}_{I}=0 .
\end{array}
$$

DaB der Bruttomietzins und der Bruttopreis für Bauleistungen die Bedingungen (III.81) und (III.84) erfüllen, ist offensichtlich. Daß auch der Bruttohäuserpreis bei gegebenem Nettohäuserpreis proportional zum Mehrwertsteuersatz steigt, wird deutlich, wenn man sich an die Definitionsgleichung für den Schattenpreis $\mathrm{P}_{\mathrm{H}}$ des Wohnflächenbestandes erinnert. Unter Beachtung von (III.8 1) gilt dann

(III.6 5)

$$
\begin{aligned}
P_{H}^{b}\left(t_{0}\right)=\frac{\partial M_{W}\left(t_{0}\right)}{\partial H\left(t_{0}\right)} & =\int_{t_{0}}^{\infty}\left(1+\tau_{u}\right) z m \cdot \exp [-z(r+)(t-t *)] d t \\
& =\left(1+\tau_{u}\right) P_{H} .
\end{aligned}
$$


DaB die Beziehung (III.83) ebenfalls erfüllt ist, ist weniger offensichtlich, ist doch der Verkauf von baureifen Grundstücken umsatzsteuerfrei. Es gelingt dem Wohnungsinvestor jedoch, die Last der von thm zu zahlenden Umsatzsteuer (abzüglich der zurückerstatteten Vorsteuerbeträge) in vollem Umfang auf den Bodenmakler zurückzuwälzen, der seinerseits keinen Anlaß sieht, seinen ursprünglichen Bodenangebotsplan unter dem Einflus dieser Uberwälzung zu ändern: auch nach Steuer ist jener Angebotsplan der zu elner Bodenpreissteigerungsrate in Höhe des zinssatzes führt, der erlösmaxlmale (dies geht aus (III.83) unter Beachtung der Laisser-Falre-Optimalitätsbedingung (II.59) hervor). Dieses eben beschriebene Glelchgewicht ist aufgrund der in Kapitel II gezeigten Eindeutigkeit der Lösung zugleich das einzig mögliche.

Eine allgemeine Umsatzsteuer (mit Vorsteuerabzug) ist allokationsneutral.

Diese Neutralität wird anhand eines einfachen Beispiels verständlich: angenommen, ein Wohnungsinvestor verausgabt für den Kauf eines Grundstücks und die Errichtung eines Gebäudes darauf insgesamt 1 Mio. D-Mark; der Einfachheit halber wollen wir eine Finanzierung mit Eigenkapital unterstellen und von abnutzungsbedingten Wertminderungen des Gebäudekapitals absehen. Jetzt und in allen Folgeperioden sei es dem Wohnungsinvestor möglich, aus der Vermietung seines Wohnungseigentums einen jährlichen Mietertrag in Höhe von 50000 D-Mark $\mathrm{zu}$ ziehen. Die Rendite der Wohnungsinvestition beträgt, wenn man von Wertsteigegerungen des Immobilienvermögens absieht, somit in jeder Periode 5 prozent. Wird in dieser situation eine allgemeine Umsatzsteuer mit Vorsteuerabzug eingeführt - Ausgabenvolumen und -struktur der privaten Haushalte bleiben unter den getroffenen Annahmen dann unverändert - und beträgt der entsprechende steuersatz z.B. 10 Prozent, so verringern sich zwar die Mieterträge um 5000 D-Mark; es sinken aber auch die effektiven Baukosten (infolge des Vorsteuerabzugs) und die Kosten für das Grundstück (infolge der Steuerüberwälzung) um denselben Prozentsatz. Die 
Rendite des in das Grundstück investierten Vermögens ist deshalb nach Einfuhrung der Steuer dieselbe wie zuvor, nämlich 5 prozent.

In Kenntnis der zusammenhänge um die Investitionsneutralität der allgemeinen Umsatzsteuer lassen sich nun auch leicht die allokativen Verzerrungen beschreiben, die infolge des Ausklammerns der Mietumsätze aus der Umsatzbesteuerung entstehen: - wïrde man sich auf die partialanalytische Betrachtungsweise der vorangegangenen Kapitel beschränken, so würde nichts passieren. Die Laisser-Falre-Bedingungen (II.54) bis (II.59) blieben dieselben; lediglich die darin enthaltenen Preise müBten nun wieder als Bruttopreise interpretiert werden;

- allokative Verzerrungen werden aber erkennbar, wenn man den partialanalytischen Rahmen verläBt und den in Abb.1 skizzierten Zusammenhängen des Totalmodells Rechnung trägt. Auf diese Welse wird es mögllch, steuerinduzierte verzerrungen in der Konsumstruktur der privaten Haushalte zu berücksichtigen: wie uns aus der mikroorkonomlschen Haushaltstheorie bekannt ist, bestimmen die Haushalte die Struktur ihres Warenkorbes unter $\mathrm{zu}-$ grundelegung der zu zahlenden Bruttopreise. Während sich der Einkaufspreis des alternativ konsumierbaren Gutes $C$ infolge der Steuerbefrelung der Mletumsätze nicht verändert, sinkt der Bruttomietpreis. Die Haushalte werden in dieser situation bestrebt setn, thren Konsum an Wohnleistungen zulasten des von der Mehrwertsteuer erfaßten Konsumgutes auszudehnen.

Da im Entscheidungsproblem des Wohnungsinvestors die Nettomiete die relevante Ertragsgröße und somit auch die bestimmende Variable bei der Berechnung des Häuserpreises $P_{H}$ ist, äuBert sich die steuerbedingte Anderung des Nachfrageverhaltens der privaten Haushalte graphisch in einer Linksverschiebung der durch Bedingung (II.57) implizit beschriebenen Nachfragekurve - bei gegebener Nettomiete (und gegebenem Häuserpreis) werden die Haushalte im Anschluß an die Umsatzsteuerbefreiung bereit sein, ihre Nachfrage nach Wohnleistungseinheiten auszudehnen. In Abb.12 ist diese Linksverschiebung der Nachfragekurve festgehalten. 
Abb. 12

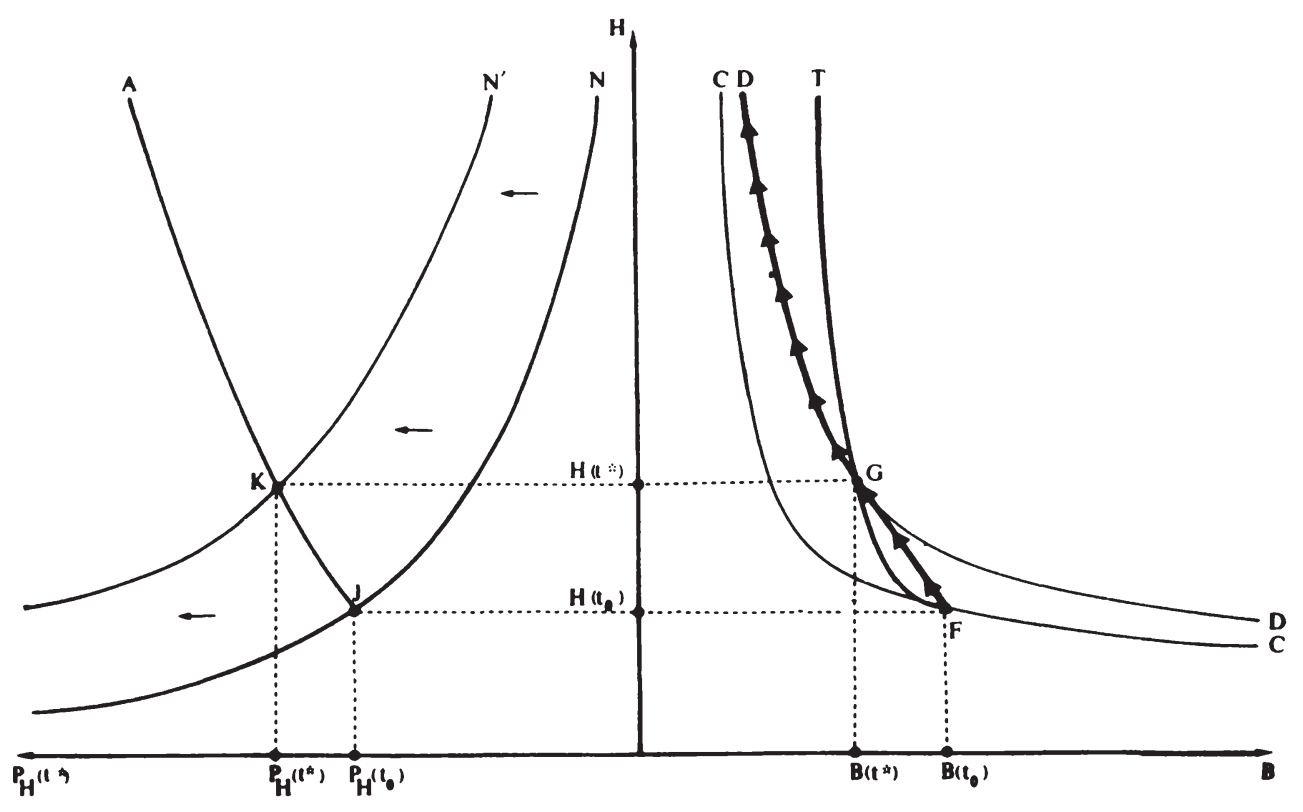

Wie Abb.12 zu entnehmen ist, reagieren die Wohnungsinvestoren auf die steuerinduzierte Nachfragesteigerung nach Wohnleistungen mit einer Intensivierung ihrer Neubautätigkeit es kommt infolge der umsatzsteuerbefreiung zu einem kurzfristigen Boom am Wohnungsneubaumarkt.

Da die gleichgewichtigen Wachstumsraten der Laisser-Faireökonomie unter dem Regime einer Mehrwertsteuer geltenden Rechts ihre Werte nicht verändern, hat die Anpassungsreaktion zur Folge, das der Häuserbestand zu jedem Zeitpunkt nach $t^{*}$ größer und der Bestand an baureifen Grundstücken zu jedem Zeitpunkt nach $t^{*} g e-$ ringer ist als in der steuerfreien welt bzw. in einer welt mit allgemeiner umsatzsteuer. 
Interessant ist es auch $\mathrm{zu}$ sehen, wie Häuser- und Bodenpreise auf die steuerbefreiung reagieren: die durch die sinkende Bruttomiete ausgelöste Zusatznachfrage nach Wohnkonsum bringt es mit sich, daß slch für den Wohnungsinvestor die Ertragsmöglichkeiten verbessern - schließlich wird er sein Wohnleistungsangebot nur dann ausdehnen, wenn seine Mieteinnahmen pro Wohnflächeneinheit zunehmen. Das neue Glelchgewicht am Markt für Wohnleistungen läßt sich deshalb durch ein erhöhtes Angebot an solchen Leistungen bei gleichzeittg gestiegener "Netto-" Miete, aber gefallener Marktmiete, beschreiben. Folgerichtig nimmt auch der Häuserpreis $P_{H}$ als Barwert der Nettoerträge $z u-K$ in Abb.12 symbolisiert das neue Marktgleichgewicht.

Der oben beschriebene Neubauboom kommt allerdings nur dann zustande, wenn die Bodenmakler bereit sind, in $t_{0}$ zusätzliches Bauland aus ihrem Bestand abzugeben. Dies wird nur dann der Fall sein, wenn die Wohnungsinvestoren ihrerselts willens sind, in $t_{0}$ einen höheren Preis $P_{B}$ je Flächeneinheit Bauland zu entrichten. Daß sie dazu bereit sind, hat seinen Grund darin, daB mit den Ertragsmöglichkeiten der Wohnungsinvestition zugleich auch die Wertgrenzprodukte der bei der Produktion von Wohnflächeneinheiten verwendeten Produktionsfaktoren zunehmen.

Die Umsatzsteuer geltenden Rechts hat damit zur Folge, daB zwar der Zeitpfad der Marktmieten unterhalb jenes Niveaus liegt, das sich im Falle einer allgemeinen umsatzsteuer eingespielt hätte, der zeitpfad des Bodenpreises $P_{B}$ sich aber ebenso dauerhaft über seinem Laisser-Faire-Niveau bewegt. Entsprechend ist auch die Kapitalintensität der Bebauung zu jedem Zeitpunkt jenseits von $t^{*}$ gröber als in der steuerfreien welt.

Wie schon bei der Analyse der Grundsteuer und der Vermögensteuer, so lassen auch die hier gefundenen Ergebnisse den Schluß zu, daß das von politischer seite so oft beklagte "zu hohe" Niveau der Baulandpreise seine Ursache nicht unbedingt in der spekulativen Zurückhaltung von Bauland haben muB. Einen anderen Erklärungsansatz für "zu hohe" Baulandpreise liefert uns der oben beschriebene Sachverhalt, daß auch das geltende 
Umsatzsteuerrecht die Zahlungsbereitschaft potentieller Käufer baureifer Grundstücke stärkt.

\subsection{Die Mehrwertsteueroption}

Neben der generellen Steuerbefreiung zeichnet sich die umsatzsteuerliche Behandlung des Wohnungssektors durch eine weitere Besonderhelt aus. Diese besteht darin, daß der Wohnungsinvestor - so er seine Investition vor dem 1.1 .1985 getätigt hat den AusschluB vom Vorsteuerabzug dadurch vermeiden kann, daß er als Vermieter auf die steuerbefreiung seiner Mietumsätze nach $\$ 9$ UStG wirksam verzichtet. Dabei hat der Gesetzgeber die Option auf den Verzicht auf die Mehrwertsteuerbefreiung an die Erfullung einer speztellen Bedingung geknüpft: denn Voraussetzung für die Inanspruchnahme der Mehrwertsteueroption ist, das die Vermietung an einen anderen Unternehmer für dessen Unternehmung erfolgt. Dieser zweite Unternehmer, der sogenannte "gewerbliche Zwischenmieter", vermietet das objekt dann seinerseits an den privaten wohnungssuchenden weiter. Für dieses zweite Mietverhältnis scheidet die Option aus; der Zwischenmieter kann die ihm vom Eigentümer auf den Mietzins in Rechnung gestellte Umsatzsteuer deshalb auch nicht als Vorsteuer abziehen. Wenn man darüberhinaus bedenkt, daB der Wohnungseigentümer durch das Eingehen eines Zwischenmietverhăltnisses lediglich zuvor von ihm selbst durchgeführte Aktivitäten delegiert, deren Kosten, obwohl nicht pekuniarisiert, in die Rentabilitätsrechnung Eingang gefunden haben, wird man davon ausgehen können, daB die Einschaltung eines gewerblichen $\mathrm{Zwischenmieters}$ keinen EinfluB hat auf das Angebot an Wohnleistungen respektive die Höhe der Marktmiete.

Aus diesem Grunde wird auch die Inanspruchnahme der Option auf Mehrwertbesteuerung für sich genommen noch keine allokativen Folgen nach sich ziehen. Wie wir in unserem obigen Fallbeispiel zeigen konnten, bleibt die Rentabilität der Wohnungsinvestition bei gegebenen Marktmieten von der Umsatzsteuer 
unberührt. Voraussetzung dieser Neutralitätseigenschaft des geltenden Umsatzsteuerrechts ist allerdings, daß die Mieteinnahmen über die gesamte Nutzungsdauer der Wohnung hinweg der Umsatzsteuer unterworfen werden. Genau diese Voraussetzung erfüllt das deutsche Umsatzsteuerrecht aber nicht: ein Wohnungsinvestor, der die Optionsmöglichkeit des $\$ 9$ UStG nutzt, muß die Mieterträge seiner Wohnungsinvestition nur in den darauf folgenden zehn Jahren versteuern. Nach Ablauf dieser Frist kann die Option steuerunschädlich widerrufen werden. Diese Regelung verhilft dem Wohnungsinvestor zweifellos zu Rentabilitätsvorteilen - von einer Gleichstellung der Produktion von Wohnleistungseinheiten gegenüber anderen mehrwertsteuerpflichtigen Aktivitäten, zu der die Mehrwertsteueroption nach dem willen des Gesetzgebers beitragen sol1 ${ }^{63}$ ) kann deshalb auch nicht gesprochen werden.

So überrascht es denn auch nicht, daß Wohnungsinvestoren in der Vergangenheit kaum ein Mittel unversucht lieben, um in den Genuß der Vorteile zu gelangen, die die Mehrwertsteueroption bietet. Daß sie dabei nicht immer im Sinne des Gesetzgebers vorgingen, zeigt das Echo, das Zwischenmietverhältnisse (vor allem im Rahmen von Bauherrenmodellen) in der öffentlichen Diskussion gefunden haben. Nach dem Willen der Legislative muß der Zwischenmieter im Rahmen der Entfaltung seiner Tätigkeit eine eigene ökonomische Funktion erfüllen; er muß ein eigenes Unternehmerrisiko, aber auch eigene Unternehmenschancen haben. Die Idendität von Wohnungsinvestor und Endmieter oder auch entsprechende Mietvereinbarungen zwischen Ehegatten, beides in der Vergangenheit vorzufindende Gestaltungsformen, entsprechen diesem willen sicherlich nicht; auch die organschaft von wohnungsinvestor und $\mathrm{Zwischenmieter} \mathrm{oder} \mathrm{ein} \mathrm{Treuhandverhältnis}$ zwischen diesen beiden Funktionsträgern würden den Intentionen des Gesetzgebers zuwiderlaufen.

63) Vgl. FG Miinster, Urteil vam 3.9.1975, EFG 1976, S.110 ff., FG Disseldorf, Urteil vam 19.1.1981, EFG 1981, S.645 f., OFD Koblenz, Verfügung von 2.3.1979, UStR 1979, S.92 f. 
Wie die Erfahrung jedoch zeigt, sind die Grenzen für solche Gestaltungsmißbräuche von den Finanzbehörden ebenso schwer zu ziehen wie von den betroffenen Vertragspartnern selbst. So hat die aus umsatzsteuerlicher sicht ungenügende Gestaltung von Zwischenmietverhältnissen oft nicht den bösen willen der beiden Vertragsparteien zum Ausgangspunkt, sondern ein Defizit an juristischer Detailkenntnis. Kennzeichnend für einen gravierenden Mangel an Rechtssicherheit ist zudem auch die Tatsache, daß gegen nahezu alle Ablehnungsbescheide der Finanzbehörden momentan Revisionen laufen, über die bis heute (Mitte 1984) aber ausnahmslos noch nicht entschieden wurde.

Die mit der Mehrwertsteueroption einhergehenden Probleme haben im Laufe der zeit so an Gewicht gewonnen, daß sich auch der Gesetzgeber gezwungen sah, Überlegungen hinsichtlich einer Reform der umsatzsteuerlichen Behandlung des Wohnungssektors anzustellen. Doch so verständlich die Motive für solche Uberlegungen sind, so falsch sind, zieht man den normativen Maßstab unserer Analyse zur Bewertung heran, die Schlußfolgerungen, die daraus gezogen wurden. Unter dem Gesichtpunkt der gesellschaftlichen Wohlfahrt wäre eine steuer begrüßenswert, die alle Umsätze und Eigenverbräuche jeder Art trifft - also auch die im sektor der Wohnleistungsproduktion getätigten. Stattdessen wurde durch den Artikel 36, Ziffer 2, Satz 2 des Zweiten Haushaltsstrukturgesetzes vom 22.12.1981 die generelle Umsatzsteuerbefreiung des Wohnungssektors bestätigt. Ein Verzicht auf die Umsatzsteuerbefreiung ist danach bei der Vermietung eines Grundstücks nach dem 1.1.1985 nur noch dann möglich, wenn das Grundstück nicht dauerhaft Wohnzwecken dient; bei allen bis zum 31.12.1984 fertiggestellten objekten bleibt die optionsmöglichkeit auch über diesen zeitraum hinaus erhalten. ${ }^{64)}$

64) Man sollte diese Neuregelung nicht als lex specialis gegen Bauherrenmodelle interpretieren. Denn wer Hotels, Appartment- oder Ferienhäuser errichtet und an einen Hotelunternehmer venmietet, kann auch weiterhin optieren und den Vorsteuerabzug in Anspruch nehmen. Die Bauherrermodelle in der Hotel- und Ferienappartmentbranche sind samit von der Neuregelung des $\$ 9$, Satz 2 UStG auch nach dem 31.12.1984 nicht betroffen. Es ist deshalb zu erwarten, daß zwar der Beitrag von Bauherrenmodellen zum Mietwohnungsbau zurückgeht, im Rahmen der eben angesprochenen Dienstleistungssektoren aber steigt. 
Zur Verteidigung der Reform des $§ 9$ UStG wird das Argument vorgebracht, daß der Gesetzgeber im Hinblick auf verteilungspolitische zeilsetzungen bereit war, die mit der steuerbefreiung des Wohnungssektors einhergehenden Wohlfahrtsverluste in Kauf zu nehmen. Und auf den ersten Blick scheint die Reform zur Verwirklichung dieser Absichten auch tatsächlich beitragen zu können, sinkt doch infolge der Umsatzsteuerbefreiung des Wohnungssektors das Niveau der Marktmieten auf Dauer. Eine etwas genauere Betrachtung zeigt aber, daß diese in die Umsatzsteuerreform gesetzten Hoffnungen trügen:

- zum einen profitieren von der steuerbefreiung nicht nur die (im Zweifelsfall ärmeren Mieter), auch die (im Zweifelsfall besser begüterten) Wohnungsinvestoren werden von der Reform begünstigt. Welche Gruppe aus der Steuerbefreiung den größeren Vorteil zieht, läßt sich a priori nicht sagen;

- aber selbst wenn man einmal unterstellt, daß die Mieter von der Reform am meisten profitieren, melden sich Bedenken an: es gehört zum ökonomischen standardwissen intergraduierter studenten, daß eine spezielle Verbrauchsteuer(befreiung) einer allgemeinen Verbrauchsteuer(befreiung) bzw. einem entsprechenden Einkommenstransfer unter wohlfahrtstheoretischen Aspekten immer unterlegen ist. 
5. Die Besteuerung der Erträge aus Wohnimmobilienvermögen. Wie wir gesehen haben, lassen die Substanzbesteuerung des Grundstückvermögens sowie die Besteuerung des Grundstücksverkehrs und die der Mjetumsätze einige wesentliche Wünsche offen, die man im Interesse einer effizienten intertemporalen Allokation der Faktoren Kapital und Boden hegen sollte. Hinsichtlich der im zuge der ertragsteuerlichen Behandlung des Wohnungssektors auftretenden Unzulänglichkeiten sind diese Mängel aber fast schon vernachlässigbar gering. Daß dieses Urteil keineswegs $z u$ scharf formuliert ist, werden wir anhand der folgenden Untersuchungen belegen können.

5.1. Ein allokationsneutrales System der Einkommensbesteuerung. Eine reine Wirkungsanalyse muß solange unbefriedigend sein, solange sie nur auf bestehende Mängel hinweist und keine Lösungsvorschläge parat hat, mit deren Hilfe man die gefundenen Mängel beseitigen kann. Die Basis einer konstruktiven Kritik des geltenden, den Wohnungssektor betreffenden Einkommensteuerrechts kann deshalb nur der Nachweis sein, daß eine allokationsnelitrale Einkommensbesteuerung des Wohnungssektors tatsächlich auch vorstellbar ist. Nur auf der Grundlage eines solchen Idealtypus' lassen sich

- Versäumnisse des Gesetzgebers nachweisen, ohne sich damit gleichzeitig den Vorwurf einzuhandeln, mit der Forderung einer effiziente(re)n Einkommensbesteuerung des Wohnungssektors Unmögliches zu verlangen;

- Lösungsvorschläge modellieren, die - so sie schon nicht mit ihm identisch sein können - dem Ideal doch zumindest nahekommen.

Auf der Suche nach einer allokationsneutralen Form der Einkommensbesteuerung stößt man spätestens dann auf Schwierigkeiten, wenn man vor der Aufgabe steht, den Begriff des steuerbaren Einkommens näher zu definieren. In der finanzwissenschaftlichen Literatur bieten sich hierzu zwei Konzeptionen an, die der quellentheorie und die der Reinvermögensugangstheorie. 
Die Quellentheorie definiert als Einkommen jene Zuflüsse an ökonomischen Werten, die regelmäßig aus einer bestimmten Quelle sprudeln; das Einkommen ist definiert als Gesamtheit derjenigen Wirtschaftsgüter, "welche alljährlich dem Einzelnen aus dauerhaften Erwerbsquellen zufließen."65)

Nach Maßgabe der Theorie des Reinvermögenzugangs wird das Einkommen umfassender definiert; danach ist Einkommen jeder zuwachs an ökonomischer Dispositionsmacht, unabhängig von der Periodizität des Zuflusses und der Art seiner Entstehung.

Welche dieser beiden Konzeptionen die für uns zweckmäßigere ist, hängt $\mathrm{ab}$ von der mit der Einkommensbesteuerung verfolgten $\mathrm{ziel-}$ setzung. Stimmt man darin überein, daß es die Aufgabe der Einkommensteuer 1st, den Finanzierungsbedarf der öffentlichen Haushalte auf der Grundlage der individuellen Leistungsfähigkeit der steuerpflichtigen zu decken, so kommt für die Abgrenzung des steuerpflichtigen Einkommens nur die Konzeption des Reinvermögenzugangs in Frage; denn so unbestimmt die Vorstellungen über eine "gerechte" Besteuerung sonst auch sein mögen - hinsichtlich eines punktes wird man sich jederzeit einigen können: daß es ungerecht wäre, wenn Arbeitseinkommen der Besteuerung unterworfen, nicht-periodische Erträge wie etwa (realisierte) Wertzuwächse des Vermögens dagegen entweder nicht in vollem Umfang oder gar nicht zur Einkommensbesteuerung herangezogen würden.

Wir wollen uns auf diese auf SCHANZ zurückgehende Definition des Einkommensbegriffs im Rahmen unserer Analyse berufen. (Ganz im SCHANZschen Sinne zählen wir zum steuerpflichtigen Einkommen des Wohnungsinvestors

65) FUISTING(1907), S.57 f.

66) Vgl. SCHANZ (1896); im angelsächsischen Bereich wurde die SCHANZsche Auffassung vor allem von SIMUNS und HAIG vertreten;- vgl. HAIG(1921), SIMONS (1938) . 
- die aus der Nutzung von Wohnungseigentum fließenden Mietumsätze in Höhe von mH. Die Wendung "Nutzung von Wohnungseigentum" wurde hierbei bewußt gewählt, denn es ist für das zustandekommen eines Zuwachses an ökonomischer Dispositionsmacht unerheblich, ob der Wohnungsinvestor sein Wohnungseigentum vermietet und die ihm hieraus zufließenden Mieterträge zur freien Verwendung hat oder ob er sein Wohnungseigentum selbst nutzt und die dadurch "gesparten", andernfalls für Mietzahlungen fällig gewordenen Geldbeträge anderweitig verwenden kann:

- die aufgrund sich im Zeitablauf verändernder Knappheitsrelationen und Nachfrageverhältnisse zustandekommenden Wertzuwächse des Häuserbestandes $\mathrm{H}$. Bezeichnen wir mit $\dot{\mathrm{P}}_{\mathrm{H}}$ die Veränderung des Häuserpreises zu einem beliebigen Zeitpunkt, so kennzej.chnet das Produkt $\dot{\mathrm{P}}_{\mathrm{H}} \mathrm{H}$ die Höhe dieser Vermögeszuwächse zu diesem zeitpunkt. Hierbei ist zu beachten, daß die Wertzuwächse schon zum Zeitpunkt ihres Entstehens (und nicht erst zum Zeitpunkt ihrer Realisierung) die Leistungsfähigkeit des Wohnungsinvestors stärken, indem sie sein beleihungsfähiges Vermögen mehren.

Abzuziehen von diesen beiden Ertragspositionen sind nach SCHANz 67 )

- "alle Schuldzinsen", die wir im Rahmen unserer Analyse mit rD, dem Produkt aus Marktzinssatz und Fremdkapitalbestand, bezeichnet haben und

- "alle Vermögensverluste". Vermögensverluste treten in unserer Modellwelt in Gestalt abnutzungsbedingter wertminderungen des Wohnflächenbestandes $\mathrm{H}$ auf: aufgrund der periodischen Substanzminderung des Gebäudekapitalstocks $K$ in Höhe von $\delta K$ scheiden in jeder Periode $\alpha \delta \mathrm{H}$ Wohnflächeneinheiten aus dem $\mathrm{Be}-$ stand $\mathrm{H}$ aus. Bewertet man diese Verluste mit dem Preis $\mathrm{P}_{\mathrm{H}}$ je Wohnflächeneinheit, so erhält man einen periodenbezogenen abnutzungsbedingten Vermögensverlust in Höhe von $\mathrm{P}_{\mathrm{H}} a \delta \mathrm{H}$-Mark.

67) SCHANZ (1896), S. 24 . 
Daß Fremdkapitalzinsen und abnutzungsbedingte Wertminderungen nicht zum steuerbaren Einkommen zählen dürfen, wird in der tagespolitischen Diskussion keineswegs als so selbstverständlich hingenommen, wie dies hier den Anschein haben mag. Allzuoft wird die Abzugsfähigkeit von Fremakapitalzinsen oder Abschreibungen von der Einkommensteuerbemessungsgrundlage als "Vergünstigung" fehlinterpretiert und dabei übersehen, daß diese Beträge kein Ausdruck individueller Leistungsfähigkeit sind, sondern Kosten, die bei dem Versuch entstehen, diese Leistungsfähigkeit zu erhalten bzw. zu steigern.

Aufbauend auf den ScHANZschen Uberlegungen erhalten wir für die Einkommensteuerzahllast $\mathrm{T}_{\mathrm{e}}^{\mathrm{W}}$ des Wohnungsinvestors

$$
\mathrm{T}_{\mathrm{e}}^{\mathrm{W}}=\tau_{\mathrm{e}}\left(\mathrm{mH}+\dot{\mathrm{P}}_{\mathrm{H}} \mathrm{H}-\mathrm{P}_{\mathrm{H}} \alpha \delta \mathrm{H}-\mathrm{rD}\right) ;
$$

$\tau_{e}$ ist der in unserer Analyse als proportional unterstellte Einkommensteuersatz .

Die Abgrenzung des steuerpflichtigen Einkommens des Bodenmaklers auf der Giundlage der Konzeption des Reinvermögenszugangs ist weniger aufwendig: weder fallen bei ihm Fremdkapitalzinsen an - dies hatten wir per Annahme ausgeschlossen -, noch muß er abnutzungsbedingte Wertminderungen des Baulandbestandes $B$ in seiner Kalkulation berücksichtigen. Und auch die Erlöse aus dem Verkauf von Bauboden stellen keinen Reinvermögenszugang dar, Ergebnis des Verkaufs ist lediglich eine Umschichtung vorhandener Vermögensbestände. Einkommen im Sinne der Reinvermögenszugangskonzeption stellen lediglich die infolge des immer knapper werdenden Baulandangebots und der permanent wachsenden Wohnleistungsnachfrage eintretenden Bodenpreissteigerungen dar. Steht $\dot{\mathrm{P}}_{\mathrm{B}}$ für die zeitliche Veränderung des Preises einer Flächeneinheit baureifen Bodens, so entfallen auf den Bodenmakler Einkommensteuerverpflichtungen in Höhe von

(III.87)

$$
\mathrm{T}_{\mathrm{e}}^{\mathrm{B}}=\tau_{\mathrm{e} \dot{\mathrm{P}}_{\mathrm{B}}^{\mathrm{B}}}
$$

D-Mark. 
Um zu sehen, wie eine auf der ScHANzschen Definition des Einkommens aufbauende Einkommensteuer Einfluß nimmt auf die Dispositionen von Wohnungsinvestoren und Bodenmaklern, müssen nun in einem zweiten schritt die in (III.86) und (III.87) definierten Einkommensteuerverpflichtungen in die Laisser-FairePlanungsprobleme der beiden repräsentativen Akteure integriert werden.

Unmittelbare Folge der Einkommensteuerpflicht ist, daß der Bodenmakler nun nicht mehr den gesamten periodenbezogenen Verkaufserlös $P_{B} F^{a}$, sondern nur noch den um die steuerzahlungen aus (IIJ. 87) gekürzten Betrag an die privaten Haushalte - die Boāeneigentümer - weiterleiten kann. Ubernimmt man die in $\mathrm{Ab}-$ schnitt II getroffene Arnahme, daß der Häuserpreis zu jedem zeitpunkt jenseits von $t_{0}$ größer ist als Eins und somit zu jedem dieser Zeitpunkte $y=1$ erfüllt ist, so gilt für die Entnahmen $A_{B}$ des Bodenmaklers $z u$ einem beliebigen Zeitpunkt $t>t_{0}$ die Beziehung

$$
A_{B}=P_{B} F^{a}-2 \tau_{e} \dot{P}_{B}^{B}
$$

Das Ziel des Bodenmaklers wird es nun wiederum sein, durch eine geschickte Planung seines aktuellen und zukünftigen Bodenangebots den Barwert der Verkaufserlöse nach Abzug der Einkommensteuer zu maximieren. Formal stellt sich dieses Problem deshalb wie folgt dar:

(III. 89)

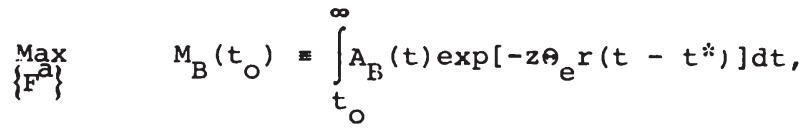

mit ${ }_{e}=1-\tau_{e}$ und unter den Bedingungen (III. 88),

$$
\text { (II.1) } \quad B\left(t_{0}\right)>0
$$

(II.17a) $z= \begin{cases}0 & \text { für } t_{0} \leq t<t^{*} \\ 1 & \text { für } t \geq t^{*}\end{cases}$

(II.45) $\quad \dot{\mathrm{B}}=-\mathrm{F}^{\mathrm{a}}$. 
Wie aus (III.89) hervorgeht, wird unter dem Regime der allgemeinen Einkommensteuer nicht mehr der Bruttomarktzinssatz $r$, sondern der Nettomarktzinssatz $\Theta \mathrm{e}^{r}$ als Diskontierungsfaktor verwendet. Der Grund hierfür liegt in der schon im Rahmen der Vermögensteueranalyse angesprochenen Funktion des Ertragswertverfahrens als Vergleichsmaßstab verschiedener, sich ausschliessender Investitionsalternativen. Diese Maßstabseigenschaft verdankt das Ertragswertverfahren dem Umstand, daß als Diskontierungsfaktor die Grenzrendite der alternativen Anlageform gewählt wird. In unserem Modell ist dies die Grenzrendite der alternativen Finanzinvestition. Wenn man bedenkt, daß auch die zinserträge aus Wertpapiervermögen der Einkommensbesteuerung unterliegen, so ist die auf die letzte in Finanzaktiva investierte D-Mark entfallende Rendite nicht mehr mit dem Bruttozins $r$, sondern mit dem im Exponenten von (III.89) stehenden Nettozins $\theta_{\mathrm{e}} \mathrm{r}$ gleichzusetzen.

Als in laufenden Werten definierte Hamiltonfunktion erhält man für das Planungsproblem (III.89) des Bodenmaklers die Gleichung

(III.9C)

$$
H=P_{B} F^{a}-z \tau_{e} \dot{P}_{B}^{B}-\lambda_{B} F^{a} ;
$$

in (III.90) steht $\lambda_{B}$ wiederum für den Schattenpreis des Bestandes an baureifen Grundstücken.

Differenziert man (III.90) nach der Kontrollvariablen $\mathrm{F}^{\mathrm{a}}$ und nach der Zustandsvariablen $B$, so errechnet man als notwendige Bedingungen für den optimalen Baulandangebotsplan

$$
\begin{aligned}
& \text { (III.91) } \frac{\partial \mathrm{H}}{\partial \mathrm{F}^{\mathrm{a}}}=\mathrm{P}_{\mathrm{B}}-\lambda_{\mathrm{B}}=0, \\
& \text { (III.92) }-\frac{\partial \mathrm{H}}{\partial \mathrm{B}}=\dot{\lambda}_{\mathrm{B}}-\mathrm{z} \theta_{\mathrm{e}} r \lambda_{\mathrm{B}}=z \tau_{\mathrm{e}} \dot{\mathrm{P}}_{\mathrm{B}}
\end{aligned}
$$

Aus (III.91) folgt, daß im Falle der inneren Lösung der Schattenpreis $\lambda_{B}$ dem Bodenpreis $P_{B}$ entspricht. Unter Berufung auf dieses Resultat folgt dann aus (III.92) 
(III. 93 a)

$$
\begin{array}{lll}
\text { (III.93a) } & \hat{\mathrm{P}}_{B}=0 & \text { für } t_{0} \leq t<t *, \\
\text { (III.93b) } & \hat{\mathrm{P}}_{B}=r & \text { für } t \geq t * .
\end{array}
$$

Die Bedingungen (III.93a) und (III.93b) informieren uns darüber, daß der Bodenmakler seinen für eine steuerfreie welt konzipierten Baulandangebotsplan im Anschluß an die Erhebung einer allgemeinen Einkommensteuer nicht ändert - (III.93b) ist identisch mit der HOTELLING-Regel der Laisser-Faire-ökonomie. Die Bodenmakler plazieren ihr Angebot an baureifen Grundstücken innerhalb des Planungshorizontes $\left(t_{0}, \infty\right)$ in der Weise, daß die dabei zustandekommende Wertsteigerung des Bodenbestandes $B$ (vor Steuer) ausreicht, die infolge der Hortung von Bauland entstandenen opportunitätskosten (vor Steuer) zu decken. Der einzelne Bodenmakler reagiert deshalb nicht auf die Erhebung der Einkommensteuer, weil er erkennt, daß er zwar durch einen vorzeitigen Verkauf von baureifen Grundstücken der Bodenwertzuwachsbesteuerung entgehen könnte, $\mathrm{da} \beta$ aber die Erträge jedes anderen Anlagemediums, in das die Bodeneigentümer die ihnen zugeflossenen Verkaufserlöse investieren könnten, ebenfalls und in gleichem Umfang zur Einkommensbesteuerung herangezogen würden. Der Barwert der Einkommensteuerzahllast der Bodeneigentümer liebe sich aus der Sicht des einzelnen Bodenmaklers durch keine der denkbaren Ausweichreaktionen verringern.

Auch im Entscheidungsproblem des Wohnungsinvestors schlägt sich der Einfluß der Einkommensbesteuerung unmittelbar in einer Verringerung des zur Entnahme anstehenden Geldbetrages nieder. Für diesen gilt die Beziehung

$$
\begin{aligned}
A_{W}= & z \Theta_{e}(m H-r D)-z\left(E-\tau_{e} \dot{P}_{H}{ }^{H}+\tau_{e} P_{H} \alpha \delta H\right)+s- \\
& -F_{\varepsilon}^{d}-P_{B} F^{d} .
\end{aligned}
$$


Der Wohnungsinvestor vertritt die Interessen der Eignerhaushalte dann am besten, wenn es ihm gelingt, durch die wahl der billigsten Finanzierungsform, des optimalen Werterhaltungsplanes und des optimalen Wohnungsneubauprogramms die Summe der Barwerte der aktuellen und aller zukünftigen Entnahmen zu maximieren. Formal beschreiben läßt sich dieses Entscheidungsproblem wie folgt:

(III.95) $\left\{\operatorname{Max}_{\text {S }, \varepsilon, F} \mathrm{~d}\right\} \quad \mathrm{M}_{W} \equiv \int_{t_{0}}^{\infty} A_{W}(t) \exp \left[-z \theta_{e} r\left(t-t^{*}\right)\right] d t$ unter den Bedingungen (III.94),

$$
H\left(t_{0}\right)<0,
$$

$$
D\left(t_{0}\right)>0
$$

(II.9)'

$0 \leq E \leq \alpha \delta \mathrm{H}$,

(II. 10)

$$
\dot{\mathrm{H}}=\mathrm{F}^{\mathrm{d}} \varphi(\varepsilon)-\mathrm{z}(\alpha \delta \mathrm{H}-\mathrm{E}),
$$

$$
S \leq P_{H}\left[F^{d} \varphi(\varepsilon)-z(\alpha \delta H-E)\right]+\dot{P}_{H} H
$$

(II.16)

$$
\dot{\mathrm{D}}=\mathrm{S}
$$

(II.17a)

$$
z=\left\{\begin{array}{ll}
0 & \text { für } t_{0} \leq t<t * \\
1 & \text { für } t \geq t^{*}
\end{array} .\right.
$$

Auch im Planungsproblem des Wohnungsinvestors dient der steuerverkürzte Marktzinssatz $\theta_{e} r$ als Diskontierungsfaktor. Die Gründe hierfür sind identisch mit jenen, die wir im Planungsproblem des Bodenmaklers genannt haben.

Mit Hilfe der oben aufgelisteten Bedingungen läßt sich für das Kontrollproblem aus (III.95) die folgende Hamiltonfunktion 
formulieren:

(III.96)

$$
\begin{aligned}
H= & z \Theta_{e}(m H-r D)-z\left(E+\tau_{e} \dot{P}_{H} H-\tau_{e} P_{H} \alpha \delta H\right)+S \\
& -F_{\varepsilon}^{d}-P_{B} F^{d} \\
& +P_{H} F^{d} \varphi(\varepsilon)-z(\alpha \delta H-E) \\
& +\lambda_{D} S .
\end{aligned}
$$

Leitet man (III.96) partiell nach der Nettoneuverschuldungsvariablen $S a b$, so erhält man als notwendige Bedingung für den optimalen Finanzplan

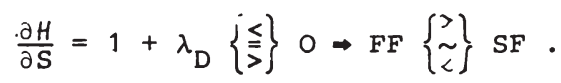

Um eine klare Aussage über die Finanzierungspräferenzen des Wohnungsinvestors machen $z u$ können, ist es offensichtlich erforderlich, den wert des Schattenpreises $\lambda_{D}$ des Fremakapitalbestandes zu kennen. Aus der Definition des Schattenpreises folgt

$$
\begin{aligned}
\lambda_{D}=\frac{\partial M_{W}}{\partial D} & =\int_{t_{0}}^{\infty}-z \Theta e^{r \exp [-z \Theta} e^{r(t-t *)] d t} \\
& =-1 .
\end{aligned}
$$

(III.98) sagt uns, daB der Marktwert des Unternehmens um eine D-Mark sinkt, wenn c.p. der Fremdkapitalbestand des Unternehmens um eine D-Mark erhöht wird. Substituiert man (III.98) in (III.97), so erkennt man, daß dort das Gleichheitszeichen und folglich für jeden beliebigen wert von $\mathrm{S}$

(III. 99) $\quad \mathrm{SF} \sim \mathrm{FF}$

gilt: auch nach der Einführung einer sich ausschließlich am Reinvermögenszugang orientierenden Einkommensteuer ist der reprä- 
sentative wohnungsinvestor indifferent bezüglich der sich ihm eröf fnenden Finanzierungsalternativen.

Dieses Neutralitätsergebnis wirft ein bezeichnendes Licht auf das vor allem in der wohnungswirtschaftlichen Praxis immer wieder $z u$ hörende Argument, daß die Abzugsfähigkeit von Fremdkapitalzinsen von der Einkommensteuerbemessungsgrundlage den Kreditnehmer dazu verführe, die Entschuldung hinauszuschieben. Dieses Argument ist falsch: durch eine solche Hinauszögerung könnte der Schuldner nichts gewinnen.

Das bezüglich der Wahl der optimalen Finanzierungsform abgeleitete Neutralitätsergebnis besitzt auch für den optimalen Werterhaltungsplan Gültigkeit. Da die in diesem Abschnitt betrachtete allgemeine Einkommensteuer keinen EinfluB hat auf die Kosten von Renovierungsarbeiten - der Preis für entsprechende Leistungen beträgt vor und nach Steuer 1 D-Mark pro Leistungseinhej.t und auch der Barwert der durch eine Leistungseinheit zusätzlich erzielbaren Mieterträge annahmegemäß den Betrag einer DMark übersteigt, werden in jeder Periode die abnutzungsbedingten Substanzminderungen des Gebäudekapitals durch entsprechende Erhaltungsaufwendungen in voller Höhe kompensiert.

Gilt diese Neutralität der Einkommensteuer auch im Hinblick auf die Neubauentscheidungen der Wohnungsinvestoren? Die Antwort auf diese Frage folgt aus der Berechnung der folgenden drei Differentiale:

$$
\begin{aligned}
& \text { (III.100) } \frac{\partial H}{\partial \varepsilon}=\frac{\partial H}{\partial \varepsilon}+\frac{\partial H}{\partial S} \frac{\partial S}{\partial \varepsilon}, \\
& \text { (III.101) } \quad \frac{\partial H}{\partial F}=\frac{\partial H}{\partial F}+\frac{\partial H}{\partial S} \frac{\partial S}{\partial F^{d}} \text {, } \\
& \text { (III.102) }-\frac{\partial H}{\partial H}=-\left[\frac{\partial H}{\partial H}+\frac{\partial H}{\partial S} \frac{\partial S}{\partial H}\right] .
\end{aligned}
$$


Infolge der Finanzierungsneutralität der allgemeinen Einkommensteuer - es gilt deshalb $\partial H / \partial S=0$ - vereinfachen sich die notwenaigen Bedingungen für den optimalen Neubauplan zu

$$
\begin{aligned}
& \text { (III. 103) } \frac{\partial H}{\partial \varepsilon}=-F^{d}+P_{H^{\prime}} \varphi^{\prime}(\varepsilon) F^{d} \text {, } \\
& \text { (III. 104) } \frac{\partial H}{\partial F^{d}}=-\varepsilon-P_{B}+P_{H} \varphi(\varepsilon)\left\{\begin{array} { l } 
{ < \} } \\
{ = j }
\end{array} 0 \Rightarrow F ^ { d } \left\{\begin{array}{l}
=\} \\
\geq j
\end{array} 0\right.\right. \text {, } \\
& \text { (III.105) }-\frac{\partial H}{\partial H}=\dot{\mathrm{P}}_{H}-z \Theta_{e} r \mathrm{P}_{H}=-\left[z\left(\Theta_{e} m-\tau e^{\dot{P}_{H}}-\Theta_{e} \mathrm{P}_{H} \alpha \delta\right)\right] .
\end{aligned}
$$

Wie man unschwer erkennen kann, hat die Einkommensbesteuerung auf die Optimalitätsbedingungen (III.103) und (III.104) keinen EinfluB. Mit Hilfe der aus Kapitel II bekannten Umformungen errechnet man sich hieraus leicht die Laisser-Faire-Bedingungen

(III.106)

$$
\mathrm{P}_{\mathrm{H}} \varphi^{\prime}(\varepsilon)=1 \text {, }
$$

(III.107) $\quad \frac{B}{\alpha} \varepsilon\left\{\begin{array}{l}<\} \\ =j\end{array} P_{B} \Rightarrow F^{d}\left\{\begin{array}{l}=\} \\ \geq j\end{array} 0\right.\right.$,

Und auch aus (III.105) läßt sich der Einkommensteuersatz mit Hilfe einiger elementarer Umformungen eliminieren. Man erhält auf diese Weise die auch für die steuerfreie Welt abgeleitete Gleichgewichtsbedingungen

$$
\begin{array}{lll}
\text { (III.108a) } & \hat{\mathrm{P}}_{\mathrm{H}}=0 & \text { für } t_{\mathrm{O}} \leq t<t *, \\
\text { (III.108b) } & \hat{\mathrm{P}}_{\mathrm{H}}=-\frac{m}{\mathrm{P}_{\mathrm{H}}}+r+\alpha \delta & \text { für } t \geq t * .
\end{array}
$$


Wir kommen damit zu dem Ergebnis, daß auch die Neubauentscheidung des Wohnungsinvestors durch die Erhebung einer allgemeinen Einkommensteuer nicht tangiert wird - auch der wohnungsinvestor erkennt, $d a \beta$ es für den Eignerhaushalt unter Rentabilitätsaspekten betrachtet keinen Unterschied macht, ob die von ihm gesparten Beträge nun in den Wohnungssektor fliessen oder anderen ertragbringenden Verwendungszwecken zugeführt werden: da im intersektoralen Gleichgewicht vor steuer alle Investitionsalternativen den gleichen Gewinn versprechen und alle diese Gewinne von der allgemeinen Einkommensteuer erfaßt und gleichmäßig belastet werden, bietet sich dem Wohnungsinvestor ebensowenig wie dem Bodenmakler eine ökonomisch sinnvolle Möglichkeit, der Last der Einkommensteuer auszuweichen. Diese Ergebnisse lassen sich zu der folgenden These zusammenfassen:

Eine allgemeine Einkommensteuer, die auf der zum ersten Mal von SCHANZ formulierten und von HAIG und SIMONS im anglo-amerikanischen Raum bekannt gemachten Reinvermögenszugangskonzeption des Einkommens gründet, ist - auch im dynamischen Sinne allokationsneutral. ${ }^{69)}$

69) Diese Neutralitätsaussage gilt wohlgemerkt nur für unseren partialanalytischen Ansatz. Hieraus den weitergehenden Schluß zu ziehen, die allgemsine Einkammensteuer sei aufgrund ihrer intersektoralen Neutralität auch paretoeffizient im Sinne des "first best", wäre ibereilt: Investitionsneutralität ist zwar eine notwendige, aber keine hinreichende Bedingung fur die globale Pareto-Effizienz eines Steuersystems. Dies wird offensichtlich, wenn man bedenkt, welchen Einfluß das oben beschriebene Steuersystem auf die Konsumentscheidung der privaten Haushalte ausibt: die Haushalte bestimmen ihren intertemporalen Konsumplan bei einer Besteuerung der Zinseinkünfte nun in der Weise, daß ihre subjektive Zeitpräferenzrate mit dem Nettorealzins $(1-\tau) r$ übereinstimmt. Einfache Arbitrageüberlegungen bestätigen dies. Da der Bruttomarktzins unter dem Regime einer allgemeinen Einkammensteuer nach wie vor mit den Nutzungskosten des im Wohnungssektor gebundenen Vermögens ïbereinstimmt, bedeutet dies, daß im Gleichgewicht nach Steuer die Zeitpräferenzrate der privaten Haushalte von diesen Nutzungskosten abweicht: die Zinseinkammensbesteuerung diskriminiert für sich genammen die gesamtwirtschaftliche Kapitalbildung, indem sie für die privaten Haushalte einen Anreiz entstehen läßt, Zukunftskonsum in die Gegenwart vorzuverlegen. 
Eine Bedingung für dieses Neutralitätsergebnis ist, daß sowohl die nachfragebedingten Wertsteigerungen als auch die abnutzungsbedingten Wertminderungen des Immobilienvermögens in voller Höhe steuerwirksam werden. Hinsichtlich der praktischen Realisierung eines solchen systems liegt hier der Einwand nahe, da $\beta$ die Differenzierung zwischen nachfragebedingten wertsteigerungen und abnutzungsbedingten Wertminderungen des Häuserbestandes zwar im Rahmen einer theoretischen Analyse möglich und dort für das Verstehen grundlegender ökonomischer Zusammenhänge auch sehr hilfreich ist, in der realen welt aber nicht vorgenommen werden kann. Es wäre aber falsch zu glauben, daß diese Unterscheidung eine für die abgeleitete Effizienz der allgemeinen Einkommensteuer notwendige Voraussetzung ist; hierfür notwendig ist allein die Bedingung, daß der in der Realität beobachtbare Saldo beider Größen, nämlich die Änderung $\left(\dot{\mathrm{P}}_{\mathrm{H}} \mathrm{H}-\mathrm{P}_{\mathrm{H}} \mathrm{a \delta H}\right)$ des Ertragswertes (Marktwertes) des Wohnimmobilienvermögens, der steuerbemessungsgrundlage zugeführt wird? diese Voraussetzung $z u$ erfüllen wäre dann nicht schwer, wenn es der Finanzverwaltung gelänge, bebaute Grundstücke zeitnah zu bewerten.

\subsection{Die Realität - die bundesdeutsche Einkommensteuer}

DaB die gegenwärtig praktizierten Verfahren der Einkommensbesteuerung auch und vor allem des Wohnungs- und des Baulandsektors diesen idealtypischen Bedingungen nicht genügen, zeigt schon ein oberflächlicher Blick auf die geltenden gesetzlichen Regelungen: so muB eine gleichmäBige Belastung aller Gewinneinkünfte schon alleine an dem Umstand scheitern, daB der Gesetzgeber in steuerlicher sicht

70) Diese Neutralitätsaussage ist keineswegs neu; so hat Johanssol, und nach ihm Samuelson (wenn auch in einem anderen Modellrahmen) gezeigt, daß die Neutralität der Einkommensbesteuerung dann sichergestellt ist, wenn neben die Erfassung aller realisierten Einkünfte und die Abzugsfähigkeit von Fremdkapitalzinsen ein Abschreibungsverfahren tritt, welches Abschreibungen pro rata temporis nach dem Ertragswertverfahren zuläßt, d.h. Ertragswertsteigerungen steuerlich als Reinvermögenszugänge und Ertragswertminderungen steuerlich als Reinvermögensabgänge interpretiert; - vgl. JOHANSSOIN (1961), SAMUETSON (1963) . 
unterscheidet zwischen den Gewinnen von Personengesellschaften und den Gewinnen von Körperschaften. Während bei Personengesellschaften einbehaltene und ausgeschüttete Gewinne mit dem persönlichen Steuersatz der Unternehmenseigner belastet werden, gilt dies bei Unternehmen mit eigener Rechtsperson seit der letzten Körperschaftssteuerreform wenigsten für die ausgeschütteten Gewinne. Die einbehaltenen Gewinne werden aber nach wie vor mit dem Spitzensatz der Einkommensteuertabelle belegt und damit in aller Regel stärker belastet als Dividenden. Trotzdem erübrigt sich eine explizite Betrachtung der Körperschaftsbesteuerung. Wie SINN (1984)nämlich zeigt, haben diese Unterschiede in der Bestelierung ausgeschütteter und einbehaltener Gewinne keinen EinfluB auf das Investitionsverhalten der betreffenden Unternehmen. Denkbare Effekte können vielmehr durch einen Wechsel der Finanzierungsform - Fremdfinanzierung oder Beteiligungsfinanzierung anstelle einer Gewinnthesaurierung - abgefangen werden. Aus diesem Grunde können wir uns in der folgenden Analyse auf die Einkommensbesteuerung von Perisonengesellschaften konzentrieren.

Aber auch diese E'ingrenzung wird unsere Analyse nicht wesentlich vereinfachen. Der weitaus größte Teil der mit der Ertragsbesteuerung des Wohnungssektors und des Baulandsektors verbundenen Probleme hat seine eigentliche Ursache vielmehr in einem grundlegenden Mangel des bundesdeutschen Einkommensteuerrechts. Dieses verzichtet nämlich auf eine allgemeine Definition des steuerbaren Einkommens. Stattdessen werden in \$2, Absatz 1 EStG sieben Einkunftsarten aufgezählt, von denen es heiBt, daß sie der Einkommensteuer unterliegen. Diese von der Tradition geprägte Unterscheidung wäre aus wohlfahrtstheoretischer Sicht unproblematisch, wenn innerhalb jeder der sieben Einkunftsarten das zu versteuernde Einkommen auf die gleiche Art und Weise - idealtypisch in der in (III.86) und (III.87) beschriebenen - bestimmt werden würde. Genau dies ist aber nicht der Fall. 
5.2.1. Die Einkommensbesteuerung des Bodenmaklers

5.2.1.1. Die Steuerfreiheit von Wertzuwächsen und das Phänomen der Baulandspekulation

Wie wir im Rahmen der wirkungsanalyse der allgemeinen SCHANZschen Einkommensteuer schon erkannt haben, sind es alleine die Wertzuwächse des Bestandes an baureifen Grundstücken, die die Dispositionsmacht der Bodeneigentümer erweitern und deshalb für eine Einkommensbesteuerung in Frage kommen. Uberraschenderweise aber übt der Fiskus bei der Besteuerung solcher Wertzuwächse äuBerste Zurückhaltung. Nur wenn das betreffende Grundstück $z u$ einem land- oder forstwirtschaftlichen Vermögen oder $z u$ einem Betriebsvermögen gehört oder wenn der Bodenmakler die Spekulationsfrist des $\$ 23$ EStG nicht beachtet, werden die entsprechenden Wersteigerungen erfaßt und entweder als

- Einkünfte aus Land- und Forstwirtschaft (§1, Absatz 1, Ziffer 1 EStG) oder als

- Einkünfte aus Gewerbebetrieb ( $\$ 1$, Absatz 1, Ziffer 2 EStG) oder als

- sonstige Einkünfte im Sinne des §22 EStG der Einkommensbesteuerung zugeführt - aber auch dann nicht zum Zeitpunkt ihres Entstehens, sondern erst zum Zeitpunkt ihrer Realisierung, a.h. erst dann, wenn das betreffende Grundstück verkauft wird.

Wie die Praxis jedoch zeigt, stellt die Besteuerung realisierter Bodenwertzuwächse eher die Ausnahme denn die Regel dar.

Man ist zunächst versucht zu glauben, daß diese faktische Steuerbefreiung realisierter Wertzuwächse baureifer Grundstücke unter Effizienzgesichtspunkten als unproblematisch zu werten ist; offensichtlich wirả der Baulandsektor im Rahmen der Einkommensbesteuerung nicht anders behandelt als in der steuerfreien Welt; entsprechend wenig wird sich deshalb auch an der 
paretoptimalen Verkaufsstrategie des repräsentativen Bodenmaklers ändern. Bei solchen Überlequngen wird aber übersehen, daß die Erträge alternativer Anlageformen sehr wohl der Einkommensbesteuerung unterliegen. Formal kommt dieser Sachverhalt dadurch zum Ausäruck, daß die Erlöse aus dem Verkauf baureifer Grundstücke zur Bestimmung des Marktwertes $M_{B}$ unter Verwendung des Nettomarktzinssatzes $\Theta_{e} r$ abdiskontiert werden. Es gilt

(III.109)

$$
M_{B}\left(t_{0}\right) \equiv \int_{t_{0}}^{\infty} P_{B}(t) F^{a}(t) \exp \left[-z \theta_{e} r\left(t-t^{*}\right)\right] d t .
$$

Wenn man nun wiederum davon ausgeht, daß sich der Bodenmakler die Maximierung des Marktwertes seines Unternehmens zum $\mathrm{Ziel}$ setzt, so läßt sich mit Hilfe des vertrauten kontrolltheoretischen Ansatzes zeigen, daß er dieses ziel nur dann erreicht, wenn er den Zeitpfad seines Bodenangebots so wählt, daß die hieraus resultierende Veränderungsrate des Bodenpreises die Bedingungen

$$
\begin{array}{lll}
\text { (III.110a) } & \hat{\mathrm{P}}_{B}=0 & \text { für } t_{0} \leq t<t *, \\
\text { (III.110b) } & \hat{\mathrm{P}}_{B}=\Theta_{e^{r}} & \text { für } t \geq t *
\end{array}
$$

erfüllt. Wie aus Bedingung (III.110 b) hervorgeht, ist die gleichgewichtige Wachstumsrate des Bodenpreises in einer ökonomie, in der die Bodenwertzuwächse baureifer Grundstücke einkommensteuerfrei bleiben, kleiner als in einer welt ohne (bzw. mit allgemeiner) Einkommensteuer. Entsprechend muß auch der Zeitpfad des Baulandangebots nun ein anderer sein. Aufgrund der steuerlichen Sonderstellung von Reinvermögenszugängen in Form von Bodenwertzuwächsen wird es für die privaten Haushalte attraktiv, Vermögen verstärkt in baureifen Grundstücken $z u$ binden. Folglich mu $\beta$ auch der im Interesse dieser Haushalte handelnde Bodenmakler bestrebt sein, einen Baulandbestand zu halten, der zu jedem Zeitpunkt 
nach Einführung der Steuer größer ist als der in einer steuerfreien welt bzw. in einer ókonomie mit allgemeiner Einkommensteuer optimale; als erstes wird er deshalb sein Baulandangebot zum Ausgangszeitpunkt $t_{\circ}$ reduzieren; diese Strategie muß bei einer gegebenen Nachfrage nach Bauland $z u$ einem Anstieg des Preises $P_{B} z u$ eben diesem Zeitpunkt führen. Andererseits wird infolge der zunächst verstärkten Hortung von Bauland das Baulandangebot zukünftiger Perioden weniger schnell schrumpfen als in der Laisser-Faire-Situation: aus Bedingung

$$
\hat{\mathrm{F}}=\hat{\mathrm{a}}-\hat{\mathrm{P}}_{\mathrm{B}}(n \beta+\alpha)=\text { konstant }<0,
$$

die auch im neuen Gleichgewicht Gültigkeit besitzt, folgt wegen (III.11Ob) die im Vergleich zur Laisser-Faire-Ökonomie geringere Wachstumsrate

$$
\left(\text { II.73) } \quad \hat{\mathbf{F}}=\hat{\mathrm{a}}-\Theta_{\mathrm{e}} \mathrm{r}(n \beta+\alpha)=\text { konstant }<0 .\right.
$$

Folge dieser steuerinduzierten Veränderung im Angebotsverhalten des repräsentativen Boaenmaklers ist, daß der Bodenpreis bei gegebenem Zeitpfad der Baulandnachfrage mit einer geringeren Rate wachsen wird als dies noch in einer welt mit allgemeiner Einkommensteuer der Fall gewesen war. Trotz des anfänglichen Anstiegs wird sich der Bodenpreis nach absehbarer zeit deshalb unterhalb seines Laissez-Faire-Niveaus bewegen.

Graphisch schlägt sich diese Planrevision des Bodenmaklers in einer Drehung der isoelastischen Entwicklungspfade aus Abb.7 nieder; wie in Kapitel II gezeigt, gilt für die steigung dieser Pfade

$$
\frac{d H}{d B}=\frac{H\left[\hat{a}-n \beta \hat{P}_{B}\right]}{B\left[\hat{a}-(n B+\alpha) \hat{P}_{B}\right]}<0 .
$$


Die steuerbedingte Abnahme der Bodenpreiswachstumsrate hat zur Folge, daß die Steigung der Entwicklungspfade zu jedem Zeitpunkt jenseits von $t_{0}$ in Absolutwerten gerechnet größer ist als im steuerfreien Fall. Dementsprechend weist der Entwikklungspfad $\mathrm{CC}^{\prime}$ in $\mathrm{Abb} .13$ in Punkt $\mathrm{F}$ - dem Zeitpunkt der Einfürung der Steuer - eine Knickstelle auf. Und auch die Sprungkurve FT' verläuft (konstruktionsbedingt) im unmittelbaren Anschluß an die erstmalige E'rhebung der Steuer steiler - auch sie knickt in $\mathrm{F}$ ein.

Es steht zu erwarten, daß die eben geschilderten Planänderungen der Bodenverkäufer auch Auswirkungen haben auf den Investitionskalkül des Wohnungsinvestors: wenn wir zunächst unterstellen, daß dieser auf die in Abschnitt III.5.1. beschriebene idealtypische Art und weise zur Einkommensteuer herangezogen wird, so läßt sich sein Verhalten mit Hilfe der dort gefundenen Gleichgewichtsbedingungen beschreiben:

$$
\begin{aligned}
& \text { (III.106) } \quad \mathrm{P}_{\mathrm{H}^{\varphi}} \varphi^{\cdot}(\varepsilon)=1 \text {, } \\
& \text { (III. 107) } \quad \frac{B_{C}}{\alpha}\left\{\begin{array}{l}
< \\
=
\end{array}\right\} P_{B} \Rightarrow F^{d}\left\{\begin{array}{l}
= \\
\geq
\end{array}\right\} 0, \\
& \text { (III.108a) } \hat{P}_{\mathrm{H}}=0 \quad \text { für } t_{\mathrm{O}} \leq t<t^{*} \text {, } \\
& \text { (III.108b) } \hat{\mathrm{P}}_{\mathrm{H}}=-\frac{\mathrm{m}}{\mathrm{P}_{\mathrm{H}}}+r+\alpha \delta \quad \text { für } t \geq t^{*} \text {. }
\end{aligned}
$$

Wie wir wissen, führt das Zurückhalten von Bauland in Periode $t_{0}$ unmittelbar $z u$ einem Anstieg der Bodenpreise. Wie aus Bedingung (III.107) hervorgeht, wird der Wohnungsinvestor auf diesen Preisanstieg mit einer Erhöhung der Kapitalintensität der Bebauung der von ihm zu erwerbenden Grundstücke reagieren. Aufgrund des abnehmenden physischen Grenzprodukts zusätzlich eingesetzter Bauleistungseinheiten - es gilt annahmegemäß $\varphi^{\prime \prime}<0$ - gelingt es ihm auf diesem Wege aber nur zum Teil, den insgesamt gestiegenen Neubaukosten auszuweichen. Unmittelbare Folge des steuerbeaingten Anstiegs der Bodenpreise 
$\mathrm{Abb} .13$

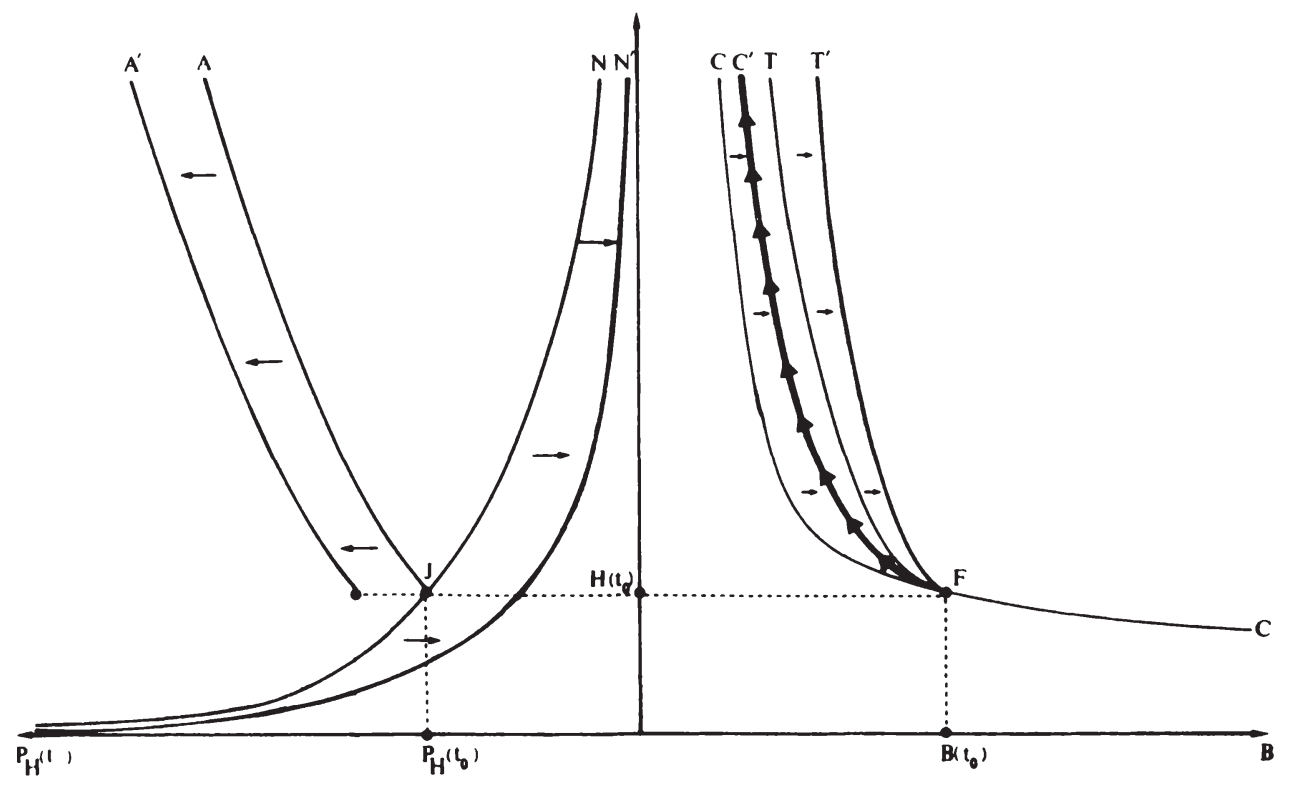

wird deshalb ein Sinken der Bereitschaft des Wohnungsinvestors sein, neue Wohnungen $\mathrm{zu}$ bauen.

Graphisch läßt sich diese gesunkene Investitionsneigung durch die in Abb.13 eingezeichnete Linksverschiebung der Angebotskurve darstellen: in Kapitel II haben wir die Tatsache, daB sich der Absolutwert des Differentialquotienten $\mathrm{dH} / \mathrm{dB}$ und der Ordinatenabstand der Angebotsfunktion gleichgerichtet verändern, in der Beziehung

(II.55)'

$$
\mathrm{P}_{\mathrm{H}}=1 / \varphi^{\prime}(\varepsilon ;
$$

$$
=1 / \varphi^{\prime}\left[\varphi^{-1}(|\mathrm{dH} / \mathrm{dB}|)\right], \mathrm{dP}_{\mathrm{H}} / \mathrm{d}(|\mathrm{dH} / \mathrm{dB}|)>0
$$

festgehalten. 
Nun sinkt in $t_{0}$ aber nicht nur die Bereitschaft des Wohnungsinvestors zum Bau neuer Wohnungen, auch die Rentabilität des gesamten, zum Zeitpunkt $t_{\circ}$ im Wohnungssektor gebundenen Vermögens nimmt im unmittelbaren AnschluB an die steuerbefreiung von Wertzuwächsen des Baulandbestandes ab. Formal schlägt sich der bei den privaten Haushalten durch die Steuerbefreiung entstandene Anreiz, ihr Portefeuille zugunsten baureifer und zulasten bebauter Grundstücke umzuschichten, in einer Verringerung der Wachstumsrate des Häuserpreises, graphisch in einer Rechtsverschiebung der Nachfragekurve nieder; aus Bedingung (III.106) folgt offensichtlich $\hat{p}_{H}=\beta \hat{\varepsilon}$, aus Bedingung (III.107) darüberhinaus $\hat{\varepsilon}=\hat{\mathrm{P}}_{B}$. Unter Berücksichtigung der modifizierten HOTELLING-Regel aus (III.110b) gilt deshalb für die Wachstumsrate des Häuserpreises

(III.111) $\quad \hat{\mathrm{P}}_{\mathrm{H}}=\beta \theta_{\mathrm{e}} \mathrm{r}$.

Da das im Wohnungssektor gebundene Vermögen infolge der Nichtbesteuerung von Wertzuwächsen baureifer Grundstücke langsamer an Wert gewinnt als unter dem Regime einer ScHANzschen Eirkommensteuer, steigen im neuen Gleichgewicht nun auch die Nutzungskosten des Wohnimmobilienvermögens; löst man Bedingung (III.108b) nach $P_{H}$ auf und berücksichtigt dabei, daß der Häuserpreis steuerbedingt langsamer wächst, so erhält man als Funktion für die Nachfragekurve $\mathrm{N}^{\prime}$ in $\mathrm{Abb} .13$ die folgende Beziehung:

$$
P_{H}=\frac{m(a / H)}{r\left(1-\beta \theta_{e}\right)+a \delta} .
$$

Vergleicht man (III.112) mit der entsprechenden Laisser-FaireFunktion, so erkennt man, daß der Quotient auf der rechten Seite des Gleichheitszeichens von (III.112) bei gegebener Nachfrage nach Wohnleistungen und gegebenem Wohnflächenbestand unter dem Einfluß der hier betrachteten steuerlichen Regelungen an Wert verliert. Entsprechend muß die das neue Gleichgewicht kennzeichnende Nachfragekurve $N^{\prime}$ rechts der Laisser-Faire-Nach- 
fragekurve $\mathrm{N}$ j.iegen.

Mit hilfe von Abb.13 lassen sich nun auch die gesuchten dynamischen Effekte der Steuerbefreiung von Wertzuwächsen baureifer Grundstücke beschreiben. Die Wohnungsinvestoren sind nicht bereit, den von den Bodenmaklern in $t_{0}$ geforderten, unter dem Einfluß der Steuer gestiegenen Bodenpreis zu bezahlen. Stattdessen stellen sie ihre Neubautätigkeit vorübergehend ein und zwar solange, bis das Wertgrenzprodukt des Bodens bei der Produktion von Wohnflächeneinheiten infolge des nachfragebedingt permanent steigenden Mietpreisniveaus wieder mit dem geforderten Bodenpreis übereinstimmt. Trotz dieses anfänglichen Neubaustopps und der dadurch ausgelösten Beschleunigung des Wachstums des Mietpreises werden jenseits eines absehbaren zeitpunktes $t * *<t *$

- der Bestand an Wohnflächeneinheiten größer und

- das Niveau der Marktmieten niedriger

sein als im Falle einer effizienten Form der Einkommensbesteuerung: die Wohnungsinvestoren antizipieren nämlich, daß die Bodenmakler langfristig bereit sein werden, ihr Angebot an baureifen Grundstücken im Vergleich zur Laisser-Faire-Welt $z u$ erhöhen. Die damit einhergehende Verbilligung der Grundstücks- bzw. Neubaukosten wird dazu führen, daß auch die Bereitschaft des Wohnungsinvestors zum Bau neuer Wohnungen im Zeitablauf zunimmt; substituiert man Bedingung (III.108b) in die Gleichung für die Wachstumsrate des Wohnflächenbestandes (II.71), so erhält man

$\left(\right.$ II. 71),$\quad \hat{\mathrm{H}}=\hat{\mathrm{a}}-\mathrm{nB \Theta}_{\mathrm{e}} \mathrm{r}$;

offensichtlich wächst der Wohnflächenbestand im neuen Gleichgewicht nach Steuer mit einer größeren Rate als dies in einer Welt mit allgemeiner Einkommensteuer der Fall gewesen war. Da sich aus (II.71)' unmittelbar auch die zeitliche Entwicklung des Wohnleistungsangebots ablesen läßt, wird infolge der steuerfreiheit von Wertzuwächsen baureifer Grundstücke bei gegebener 
Entwicklung der Wohnleistungsnachfrage auch das Mietenniveau mit einer geringeren als der paretooptimalen Rate wachsen.

Der Bestand an baureifen Grundstücken ist dagegen zu jedem Zeitpunkt jenseits von $t_{0}$ gröBer, als dies aus wohlfahrtstheoretischer sicht wünschenswert wäre. Auf formalem Wege gelangt man zu diesem Ergebnis, wenn man in die Gleichung für die Schrumpfungsrate des Baulandbestandes

$$
\hat{B}=\hat{a}-\hat{P}_{B}(n \beta+\alpha)=\text { konstant }<0
$$

die gleichgewichtige Wachstumsrate des Bodenpreises aus (III.110b) einsetzt.

\subsubsection{Die Besteuerung realisierter VeräuBerungsgewinne}

$\mathrm{DaB}$ infolge der Steuerfreiheit von Wertzuwächsen bebauter Grundstücke die so oft verdammte spekulation mit Bauland an Attraktivität gewinnt, sollte den verantwortlichen Politikern eigentlich zu denken geben. Doch gerade im politischen Raum werden immer wieder Reformvorschläge blockiert, die die steuerliche Erfassung von Bodenwertzuwächsen zum Gegenstand haben:

- so versucht man, die Praktikabilität der Erfassung von Wertsteigerungen zum Zeitpunkt ihres Entstehens mit dem Hinweis auf verwaltungstechnisch nicht lösbare Probleme $\mathrm{zu}$ widerlegen;

- aber auch die Besteuerung realisierter Wertsteigerungen als "zweitbeste" Lösung wird abgelehnt - mit dem Argument, daß der Barwert der steuerzahlungen bei einer steuer auf realisierte Veräußerungsgewinne geringer sei als bei einer steuer, die die Wertzuwächse schon zum zeitpunkt ihres Entsehens erfaBt und. somit für den Bodenmakler einen Anreiz entstehen lasse, den Verkauf von Bauland hinauszuzögern. Auf diese Weise würde die optimale Allokation des Bodens - die Wanderung des Bodens zum besten Wirt - behindert werden. 
Vor allem das zweite Argument entbehrt einer gewissen Logik: es ist sicherlich nicht sinnvoll, einen "zweitbesten" Reformvorschlag an der (angeblich?) nicht praktikablen besten Problemlösung zu messen - wer gegen die Besteuerung realisierter Wertzuwächse zu Felde ziehen möchte, muß vielmehr beweisen können, daß die Besteuerung realisierter Wertzuwächse den der geltenäen Regelung immanenten Anreiz zur Bodenspekulation noch verstärkt. Wir wollen im folgenden den Beweis antreten, daß dem nicht so ist.

Im Zuge dieses Beweises läßt sich darüberhinaus auch zeigen, daß zumindest die Ursachen der üblicherweise unterstellten differentiellen Inzidenz zwischen einer steuer auf realisierte und einer Steuer auf eingetretene Wertzuwächse durch die herrschende Meinung fehlgedeutet werden.

Bemessungsgrundlage der Steuer auf realisierte Wertzuwächse ist nun nicht mehr die Wertänderung $\dot{\mathrm{P}}_{\mathrm{B}} \mathrm{B}$ des Bestandes an baureifen Grundstücken $z u$ einem beliebigen Zeitpunkt $t$, besteuert wird vielmehr der zwischen einem bestimmten stichtag - wir wollen unterstellen, daß $t_{0}$ dieser stichtag ist - und dem Verkauszeitpunkt $t$ eingetretene Wertzuwachs des verkauften Bodenareals $F^{a}$. Der vom Bodenmakler aufzubringende steuerbetrag beläuft sich dann $z u$ einem beliebigen zeitpunkt $t$ auf

$$
T_{e}^{B}(t)=\tau_{e}\left[P_{B}(t)-P_{B}\left(t_{0}\right)\right] F^{a}(t) .
$$

Unter Berücksichtigung von (III.113) präsentiert sich das Planungsproblem des Bodenmaklers dann wie folgt:

(III.114)

$$
\operatorname{Max}_{\left.F^{a}\right\}} \quad M_{B}\left(t_{0}\right) \equiv \int_{t_{0}}^{\infty}\left[P_{B}(t) F^{a}(t)-T_{e}^{B}(t)\right] \exp \left[-z \theta_{e} r\left(t-t^{*}\right)\right] d t .
$$

unter den Bedingungen (II.1), (II.17a) und (II.45).

Die Hamiltonfunktion für dieses Problem lautet, formuliert in laufenden Werten,

(III.115)

$$
H=\Theta_{e} P_{B} F^{a}+z \tau_{e} P_{B}\left(t_{0}\right)-\lambda_{B} F^{a} \text {. }
$$


Durch die partielle Differentiation von (III.115) nach der Kontrollvariablen $\mathrm{F}^{\mathrm{a}}$ und der Zustandsvariablen $\mathrm{B}$ erhält man als notwendige Bedingungen für den optimalen Verkaufsplan im Falle einer inneren Lösung

(III.116)

$$
\lambda_{B}=\left(1-z \tau_{e}\right) P_{B}+z \tau_{e} P_{B}\left(t_{0}\right)
$$

und

(III. 117)

$$
\dot{\lambda}_{B}-z \Theta_{e} r \lambda_{B}=0
$$

Bedenkt man, daß aus (III.116) - wegen der Konstanz von $\mathrm{P}_{B}\left(t_{0}\right)-\dot{\lambda}_{B}=\left(1-z \tau_{e}\right) \dot{P}_{B}$ folgt, so resultieren aus der substitution von (III.116) in (III.117) die Bedingungen

(III.118a)

$$
\hat{\mathrm{P}}_{\mathrm{B}}=0
$$$$
\text { für } t_{0} \leq t<t *
$$

(III.118b)

$$
\hat{P}_{B}=\theta_{e} r+\tau e^{r \frac{P_{B}\left(t_{O}\right)}{P_{B}}} \leq r \text { für } t \geq t^{*} \text {. }
$$

Mit Blick auf (III.118) lassen sich die gesuchten differentiellen Inzidenzen

- zwischen einer Einkommensteuer, die Wertzuwächse baureifer Grundstücke überhaupt nicht erfaßt, und einer Einkommensteuer, die Wertsteigerungen zum Zeitpunkt ihrer Realisierung belastet sowie

- zwischen einer Einkommensteuer, die lediglich Veräußerungsgewinne belastet, und einer solchen, der Wertsteigerungen schon zum Zeitpunkt ihres Entstehens zugeführt werden ableiten.

Offensichtlich ist die Bodenpreissteigerungsrate im Falle der Besteuerung realisierter Bodenwertzuwächse größer als in dem Fall, in dem auf die steuerliche Erfassung dieser Wertzuwächse ganz verzichtet wird. Nur für $t \rightarrow \infty$ stimmen die Preis- 
steigerungsraten wegen $\lim _{t \rightarrow \infty} \mathrm{P}_{\mathrm{B}}=\infty$ in beiden Fällen überein. Dieses Ergebnis zeigt uns, das der infolge der Nichtbesteuerung von Rodenwertzuwächsen gestiegene Anreiz zur Hortung von Bauland durch eine Besteuerung der realisierten Bodenwertzuwächse gemindert wird. Die als Folge dieser geänderten Strategie eintretende Verknappung von Bauland in zukünftigen Perioden muß bei gegebener Entwicklung der Nachfrage nach Bauland zu einem beschleunigten Wachstum des Bodenpreises führen. Den Schlüssel zur ökonomischen Interpretation dieser steuerinduzierten Änderung im Angebotsverhalten des Bodenmaklers finden wir, wenn wir uns daran erinnern, daß die Erhebung einer Grunderwerbsteuer in Form einer Nutzungsänderungssteuer die Planungen der Bodenmakler nicht tangiert (vgl. Kapitel III.3. dieser Arbeit). Von dieser Steuer unterscheidet sich die Steuer auf realisierte Wertzuwächse lediglich dadurch, daß sie neben der zwischen dem stichtag $t_{0}$ und dem Verkaufszeitpunkt $t$ eingetretenen Wertsteigerung nicht auch noch den zum Stichtag gültigen Bodenpreis $P_{B}\left(t_{0}\right)$ des Grundstücks belastet. Steuerwirkungsanalytisch betrachtet ist eine steuer auf realisierte Bodenwertzuwächse demnach gleichzusetzen mit einer - neutralen Nutzungsänderungssteuer zuzüglich eines festen, zum Verkaufszeitpunkt ausgezahlten Transfers in Höhe von $\tau_{e} e_{B}\left(t_{0}\right) F^{a}$. Dieser (fiktive) Transfer ist dafür verantwortlich, daB der ursprünglich $z u$ einem bestimmten zeitpunkt $t$ geplante Grundstücksverkauf zeitlich vorgezogen wird; denn offensichtlich ist der auf den Betrachtungszeitpunkt $t_{0}$ bezogene Gegenwartswert dieses Transfers umso höher, je eher er realisiert werden kann. DaB der Bodenmakler nicht seinen gesamten Baulandbestand zum Zeitpunkt $t_{0}$ veräußert, hat seinen Grund darin, daß die aus dem vorzeitigen Verkauf einzelner Grundstücke bzw. der damit einhergehenden Verknappung von Bauland in den Folgeperioden resultierende höhere Bodenpreissteigerungsrate den Bodenmakler für die gesamten ihm entstehenden opportunitätskosten des Hortens von Bauland entschädigt; diese Opportunitätskosten umfassen nun auch die Zinsausfälle $\tau_{e} e^{r P_{B}}\left(t_{0}\right) F^{a}$, die durch den Verzicht auf einen vorzeitigen Verkauf und die Realisierung des 
damit verbundenen Transfers entstehen. Bedingung (III.118b) informiert uns auch darüber, daB der EinfluB des Transfers $\tau_{e} P_{B}\left(t_{0}\right) F^{a}$ auf die Planungen des Bodenmaklers umso geringer ausfällt, je später der Verkauf eines baureifen Grundstücks ursprünglich geplant war. Für $t \rightarrow \infty$ geht dieser EinfluB wegen $\lim _{t \rightarrow \infty} P_{B}=\infty$ gegen Null. Dieser zuletzt genannte Aspekt darf aber ilicht darüberhinwegtäuschen, daB die Einführung einer Steuer auf realisierte VeräuBerungsgewınne baureifer Grundstücke zu jedem Zeitpunkt vor $t=\infty$ den Anforderungen einer effizienten Besteuerung näher kommt als die generelle steuerliche Freistellung dieser Vermögenszuwächse.

(III.118b) ist ferner zu entnehmen, daß eine steuer auf realisierte Wertzuwächse im Vergleich zu einer allgemeinen Einkommensteuer, die Wertzuwächse schon zum Zeitpunkt ihres Entstehens erfaßt, den Anreiz zur Hortung von Bauland verstärkt. Insofern bestätigen die hier abgeleiteten Ergebnisse das Urteil, das u.a. auch der Wissenschaftliche Beirat beim Bundesfinanzministerium in seinem Gutachten zur Besteuerung von Bodenwertzuwächsen über die Inzidenz dieser Steuer gefällt hat. Grundlegende Unterschiede zur Auffassung des Beirats zeigen sich jedoch in der Begründung dieses Urteils. Das Argument des Beirats, im Rahmen einer Steuer auf realisierte Wertzuwächse sei es dem Bodenmakler möglich, durch eine zeitliche Hinauszögerung des Bodenverkaufs den Barwert der Steuerzahlungen $\mathrm{zu}$ verringern, im Rahmen einer allgemeinen Einkommensteuer mit sofortiger Besteuerung eingetretener Wertzuwächse dagegen nj.cht, ist zumindest mißverständlich. Denn die Tatsache, daß eine Steuer auf VeräuBerungsgewinne erhoben wird, ist allein noch kein ausreichender Grund, eine Hinauszögerung des Bodenverkaufs $z u$ befürchten; dies hat unsere Analyse gezeigt.

Die wahre Ursache der im Vergleich zur SCHANZschen Steuer verkaufsverzögernden Inzidenz einer steuer auf realisierte Veräußerungsgewinne ist vielmehr das Ergebnis des Aufeinandertreffens zweier gegenläufiger Effekte:

- der erste Effekt kommt dadurch zustande, daß zwar die Erträge alternativer Vermögensbestände der Einkommensteuer unterliegen, 
nicht aber die Wertsteigerungen des Bestandes baureifer Grundstücke - besteuert wird lediglich der (realisierte) Wertzuwachs der in der betreffenden Periode verkauften Grundstücke. Daß diese Steuerbefreiung das Horten von Bauland attraktiv und den Verkauf zu einem späteren Zeitpunkt rentabler macht, haben wir in Abschnitt III.5.2.1.1. gezeigt. In Bedingung (III.118b) steht der Nettomarktzinssatz $\theta_{e}$ für diesen verkaufshemmenden Effekt.

- hinter dem zweiten Effekt verbirgt sich das oben abgeleitete Ergebnis, daß eine Steuer auf realisierte Wertzuwächse, läßt man die Besteuerung anderer Einkunftsarten außer Acht, für sich genommen die spekulation mit Bauland unrentabler werden läßt. Dieses Ergebnis widerspricht zwar allen gängigen Urteilen über diese steuer, läßt sich ungeachtet dessen aber begründen, wenn man die Zerlegung dieser steuer in eine allokationsneutrale Nutzungsänderungssteuer und eine verkaufsfördernde Veräußerungsprämie vornimmt. In Bedingung (III.118b) kommt dieser verkaufsfördernde Effekt in dem Term $\tau e^{r P_{B}}\left(t_{0}\right) / P_{B}$ zum Ausdruck.

Faßt man beide Effekte zusammen, so erhält man für die unter dem Regime einer steuer auf realisierte Wertzuwächse zustandekommende gleichgewichtige Wachstumsrate des Bodenpreises

$$
\text { (III.118b)' } \quad \hat{P}_{B}=r-\tau e^{r \frac{P_{B}-P_{B}\left(t_{O}\right)}{P_{B}}} .
$$

Die Wachstumsrate $\hat{\mathrm{P}}_{\mathrm{B}}$ liegt im Fall der einkommensteuerlichen Erfassung realisierter Wertzuwächse für jeden Zeitpunkt $t \geq t *$ unter der entsprechenden Laisser-Faire-Wachstumsrate: per saldo dominiert also der verkaufshemmende Effekt der steuerbefreiung von Wertzuwächsen des Baulandbestandes.

Wir wollen für unseren weiteren Analyseweg unterstellen, daB die Wertzuwächse baureifer Grundstücke allgemein steuerfrei sind. Diese Einschränkung ist insofern unproblematisch, als sie in der steuerpraxis wohl den Regelfall darstellt. 


\subsubsection{Die Einkammensbesteuerung des Wohnungsinvestors}

Auch im Rahmen der Einkommensbesteuerung des Wohnungsinvestors wird massiv gegen das der Reinvermögenszugangskonzeption zugrundeliegende Gerechtigkeitspostulat der steuerlichen Gleichbehandlung aller Einkunftsarten verstoBen. Wie ein Blick auf die aktuellen Bestimmungen des Einkommensteuerrechts zeigt, können Einkünfte aus der Produktion von Wohnungsnutzungen in insgesamt fünf verschiedenen Einkunftsarten erfabt werden, nämlich

- als Einkünfte aus Land- und Forstwirtschaft

- als Einkünfte aus Gewerbebetrieb,

- als Einkünfte aus selbständiger Arbeit (\$21, Absatz 3 EStG),

- als Einkünfte aus Vermietung und Verpachtung nach $\S 21$ EStG und

- als Einkünfte aus Vermietung und Verpachtung nach $\$ 21$ a EStG.

Es wird dem Wohnungsinvestor keineswegs gleichgültig sein, welches der obengenannten Verfahren zur Abgrenzung des steuerpflichtigen Einkommens bel ihm zur Anwendung gelangt, weichen doch die hierbei ermittelten $z u$ versteuernden Einkünfte trotz ökonomisch identischer Vorgänge zum Teil erheblich voneinander $a b$. Es würde den Rahmen dieser Arbeit sprengen, wollte man sämtlichen einkunftsartspezifischen Unterschieden in der Gewährung von Freibeträgen, der Anwendung von Schätzverfahren zur Einkommensermittlung, der Einräumung von Werbungs- bzw. Betriebskostenabzügen etc. aufzeigen und allokationstheoretisch auswerten. Um die grundlegenden Mängel in der Einkommensbesteuerung des Wohnungssektors aufzeigen zu können, genügt es, sich auf die Analyse von drei Varianten zur Ermittlung des steuerpflichtigen Einkommens von Wohnungsinvestoren $\mathrm{zu}$ beschränken: wir wollen uns im folgenden auf die Besteuerung des durch die Nutzung von Wohnungseigentum entstandenen Einkommens im Rahmen - der Einkünfte aus Vermietung und Verpachtung nach \$21 EStG, - der Einkünfte aus Vermietung und Verpachtung nach $\$ 21 \mathrm{a}$ EStG und

- der Einkünfte aus Gewerbebetrieb konzentrieren.

Diese durch Legaldefinition postulierte Ungleichbehandlung ökonomisch gleichartiger Einkommensströme ist aber keineswegs die einzige Ursache für denkbare Wohlfahrtsverluste: im Gegen- 
satz zum Bodenmakler bietet sich dem Wohnungsinvestor zusätzlich die Möglichkeit, durch eine entsprechende Ausgestaltung der Nutzungsphase seines Wohnungseigentums selbst zu bestimmen, welche der drei oben angesprochenen Varianten der Einkommensermittlung die für ihn relevante ist. Zusätzlichen Gestaltungsspielraum eröffnet ihm hierbei die Tatsache, daß es der Finanzverwaltung bisher nicht gelungen ist, trennscharfe Grenzen zwischen den verschiedenen Einkunftsarten zu ziehen.

Unabhängig von der Wahl der Nutzungsform erhält der Wohnungsinvestor innerhalb der Erwerbsphase seines Wohnungseigentums eine weitere Chance, auf die Bemessung seiner persönlichen Einkommensteuerzahllast unmittelbar Einfluß zu nehmen. So ist es für die Ermittlung des steuerpflichtigen Einkommens von wesentlicher Bedeutung, ob der Wohnungsinvestor die Immobilie als Bauherr ${ }^{72}$ ) selbst erstellt und hierbei womöglich noch Eigenleistungen erbracht hat oder von einem Bauträger erworben hat.

Die Tatsache, daß die spätere Art der Nutzung des Wohnungseigentums unabhängig ist von der Art des Erwerbs, legt es nahe, die Analyse am chronologischen Ablauf von Anschaffung bzw. Herstellung und Nutzung auszurichten; die im Rahmen der Analyse der Erwerbsphase gewonnenen Erkenntnisse besitzen für jede der zu untersuchenden Nutzungsformen Gültigkeit.

\subsubsection{Die Einkommensbesteuerung in der Erwerbsphase -} Käufer versus Bauherr

Beim Erwerb von Wohnungseigentum fallen grundsätzlich drei Kategorien von Aufwendungen an. Dies sind

- zum ersten die nicht abzugsfähigen Anschaffungskosten des Bauplatzes einschließlich aller dazugehörenden Erwerbsnebenkosten,

- zum zweiten die Anschaffungs- bzw. Herstellungskosten des Gebäudes, die im Laufe der Nutzungsphase des Gebäudes über einen bestimmten Zeitraum hinweg in jährlichen Beträgen abgeschrieben

72) Zum Begriff des Bauherrn siehe S.10, insbesondere Fußnote 3. 
werden können und

- zum dritten die Werbungskosten, die im Jahre der Verausgabung von der Einkommensteuerbemessungsgrundlage abgezogen werden dürfen.

Einen Ansatzpunkt zur Kritik am geltenden Einkommensteuerrecht bietet die Tatsache, daB bei insgesamt gleichem Ausgabenvolumen die Proportionierung dieser drei Ausgabenblöcke unterschiedlich ausfällt, je nachdem, ob der Wohnungsinvestor sein Wohnungseigentum käuflich erworben oder als Bauherr errichtet hat bzw. auf eigenen Namen und eigene verantwortung errichten ließ. Gegenstand der Kritik sind die steuerlichen Konsequenzen, die mit der unterschiedlichen Abgrenzung einhergehen. So kann der Bauherr beispielsweise alle während der Herstellungsphase anfallenden Schuldzinsen als Werbungskosten unmittelbar steuermindernd geltend machen (wobei das Einkommensteuerrecht hierunter auch alle Aufwendungen subsumiert, die der Steuerpflichtige erbringen muß, um das Darlehen $z u$ erhalten, so beispielsweise Disagien). Wenn der Wohnungsinvestor die Immobilie dagegen käuflich erwirbt, muß er einen Großteil dieser vom Bauherrn sofort abzugsfähigen Werbungskosten über die Nutzungsphase der Immobilie hinweg abschreiben, da diese Kosten im Kaufpreis enthalten sind - so jedenfalls die übliche Argumentation.

Der Weg von dieser vermeintlich so offenkundigen Ungleichbehandlung zu der Schlußfolgerung, hier eine Quelle für Wohlfahrtsverluste entdeckt zu haben, ist nicht weit. Tatsächlich sind solche SchluBfolgerungen in der Praxis die Regel - wobei Ineffizienzen üblicherweise auf eine vermutete steuerliche Begünstigung des Bauherrn zurückgeführt werden: wenn man davon ausgehe, daß Bauherr und Käufer zwei identische Objekte zu (vor Steuer) gleichen Kosten erwerben bzw. erstellen können - so die geläufige Kritik - erhalte der Werbungskostenabzug den Charakter einer Sofortabschreibung, und der Bauherr komme deshalb im Vergleich zum Käufer in den GenuB einer zinslosen Steuerstundung. Was ist von dieser Argumentation zu halten? 
Zunächst muß darauf hingewiesen werden, daß der Vergleich zwischen Bauherr und Käufer, so wie er üblicherweise vorgenommen wird, aus ökonomischer sicht unzweckmäßig ist. Während sich hinter dem Käufer (in aller Regel) der Produzent von wohnleistungen verbirgt, wird dem Bauherrn in der Diskussion eine Doppelfunktion zugestanden: die des Bauherrn im engeren Sinne, der die wohnung errichtet bzw. errichten läßt, und die des produzenten von wohnleistungen.

Wenn man zu einem fundierten Urteil uber die Allokationseffizienz der Besteuerung während der Herstellungs- bzw. der Anschaffungsphase gelangen will, mu 3 man dem Bauherrn, wie er in der tagespolitischen Diskussion verstanden wird, neben dem Käufer einer Wohnimmobilie deshalb auch noch denjenigen gegenüberstellen, der diese Wohnung errichtet hat bzw. errichten ließ.

Folgt man diesem Vorschlag, so läßt sich zeigen, daß die zunächst plausible Vermutung einer Begünstigung des Bauherrn als Folge des alleine ihm zustehenden Werbungskostenabzugs zwar im Ergebnis nicht unbedingt falsch, in der Begründung aber zu oberflächlich ist.

Betrachten wir zur Veranschaulichung unserer Ujberlegungen $\mathrm{Abb}$. 14; die beiden Graphiken $14 \mathrm{a}$ und $14 \mathrm{~b}$ stehen für zwei auch in der Kostenstruktur vollkommen identische Investitionsobjekte; $14 \mathrm{a}$ gibt die Kostenstruktur desjenigen Gebäudes wieder, dessen Bauherr zum einen auf das Erbringen von Eigenleistungen verzichtet hat und zum anderen das objekt nach der Fertigstellung nicht zu verkaufen beabsichtigt; $14 \mathrm{~b}$ steht dagegen für den Fall, daß der Bauherr (als Bauträger beispielweise) das Gebäude nach dessen Fertigstellung an einen Produzenten von Wohnleistungen verkauft.

Unterstellen wir zunächst eine welt ohne Steuer, so müssen beide objekte, um rentabel zu sein, einen Mietertrag erwirtschaften, dessen Barwert mindestens so groß ist wie die durch die Wohnungsinvestition entstandenen Kosten. Die Strecke AD ent- 
spricht demnach dem Ertragswert der Grenzinvestition und signalisiert dementsprechend auch die maximale Zahlungsbereitschaft eines potentiellen Käufers für das betreffende objekt. Führt man nun die Einkommensteuer in unser Modell ein und berücksichtigt, daß der Werbungskostenblock im Jahr der Herstellung abzugsfähig ist ${ }^{73}$ ), vermindern sich die Gesamtkosten um das Produkt aus Einkommensteuersatz und Werbungskosten - und zwar bei beiden Bauherren. Als Folge des Werbungskostenabzugs sinkt der steuerliche Buchwert beider Gebäude auf den Betrag BC. Ob der Wohnungsinvestor, der zugleich auch Bauherr der Wohnimmobilie ist, vom Steuergesetz im Vergleich zum kaufenden Erwerber tatsächlich präferiert wird, hängt nun offensichtlich davon $a b$, ob und in welchem Maße der Bauträger bereit ist, die ihm zugutegekommene Einkommensteuererstattung im Kaufpreis weiterzugeben. Und diese Bereitschaft wird ihrerseits maßgeblich davon abhängen, ob der Bauträger realisierte Veräußerungsgewinne der Einkommensteuerbemessungsgrundlage zuführen muß oder nicht:

- handelt es sich um einen Bauträger, der realisierte Veräußerungsgewinne - die Differenz zwischen Verkaufspreis und Buchwert - nicht versteuern muß, so reicht schon ein Verkaufspreis in Höhe von $\mathrm{AE}$ aus, um die nach der steuererstattung $\left(\tau e^{\text {WK }}\right)$ verbliebenen Unkosten $z u$ decken. Bei diesem Verkaufspreis stimmen auch die Anschaffungskosten des Käufers (AE) in $\mathrm{Abb}$. 14b mit den nach dem Abzug der Werbungskosten verbliebenen Gesamtkosten des Bauherrn in Abb.14a überein. Auch die Barwerte der von beiden erwarteten Mieterträge sind annahmegemäß dieselben. Der entscheidende Unterschied zwischen beiden Erwerbsformen besteht darin, daß der Wohnungsinvestor in Abb.14a im Verlauf der Nutzungsphase nur den Restbuchwert BC des Gebäudes im Rahmen der jährlichen Absetzungen für Abnutzung steuermindernd geltend machen darf, der Käufer aus

73)Es wäre irrig, in der Abzugsfähigkeit von Werbungskosten eine spezielle steuerliche Begünstigung des Wohnungssektors zu sehen: auch bei der Produktion anderer Güter und Leistungen können Lohn-, Materialund Finanzierungskosten als Werbungskosten bzw. Betriebsausgaben einkammen- (bzw.körperschaft-) steuemindernd geltend gemacht werden. 
Abb. 14

a)

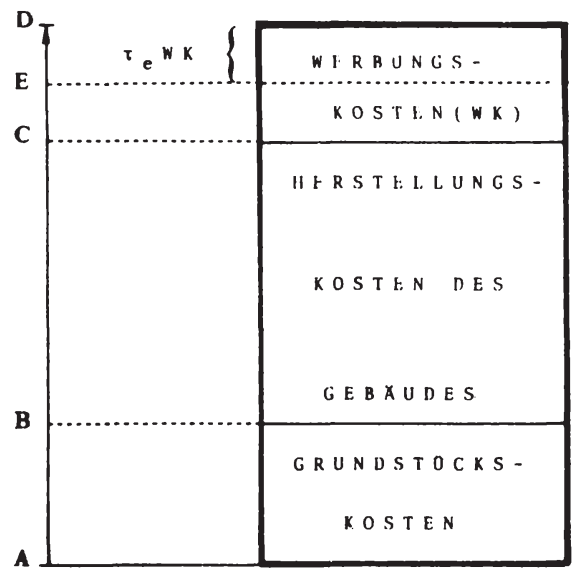

b)

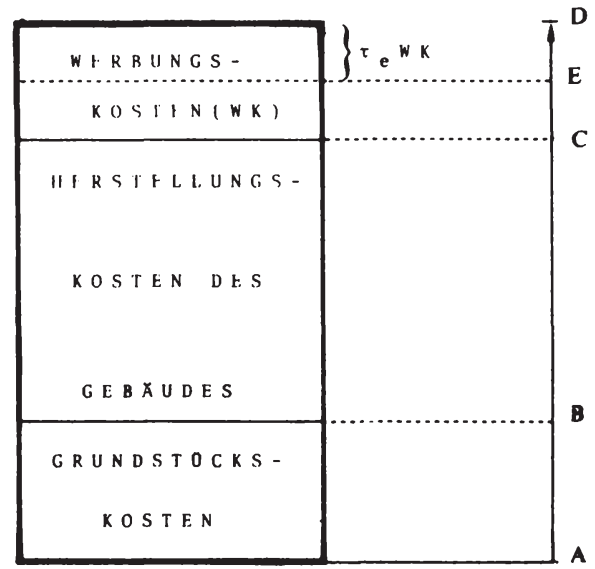

$\mathrm{Abb}$ 14b dagegen die höheren Anschaffungskosten BE. Damit erweist sich aber der Kauf vom Bauträger als die vergleichsweise billigere Erwerbsform; von einer steuerlichen Begünstigung des Bauherrn kann - zumindest in diesem Fall - nicht gesprochen werden;

- wenn der Bauträger dagegen VeräuBerungsgewinne versteuern muB, wird der Verkauf zum Preis $\mathrm{AE}$ im allgemeinen nicht zustandekommen: da der Bauträger den Betrag EC als Veräußerungsgewinn der Einkommensteuer zuführen müßte, entstünde ihm hierdurch ein Verlust in Höhe von $\tau e^{E C}$. Der Preis, der seine Kosten gerade deckt, ist offensichtlich $\mathrm{AD}$ : da von dem hierbei realisierten VeräuBerungsgewinn DC der Betrag $\tau_{e} D C=$ L'D weggesteuert wird - der ursprüngliche Werbungskostenabzug wird dadurch neutralisiert -, verbleibt dem Bauträger der Nettoverkaufserlös $A E$, der exakt seinen verbliebenen Unkosten ent- 
spricht. Der Käufer der Immobilie ist auch bereit, den Preis $A D z u$ entrichten, da der Barwert der mit Hilfe der Immobilie erzielbaren Nettomieterträge mit diesem Betrag übereinstimmt. Die Rentabilitätsnachteile dieser Konstellation gegenüber den bisher betrachteten Möglichkeiten des Erwerbs sind offensichtlich. Während der Wohnungsinvestor, der sein Wohnungseigentum von einem zur Versteuerung realisierter Veräußerungsgewinne verpflichteten Bauherrn käuflich erwirbt, die Summe BD der Anschaffungskosten des Gebäudes nur in jährlichen Beträgen im Rahmen der steuerlichen AfA absetzen kann, ist es dem Wohnungsinvestor, der sein Wohnungseigentum als Bauherr eruirbt, möglich, einen Teil dieseer Gesamtkosten sofort von der Einkommensteuerbemessungsgrundlage abzusetzen. Für diesen speziellen Fall ist das Urteil, der Bauherr werde im Vergleich zum Käufer durch die aktuellen Regelungen des Einkommensteuerrechts begünstigt, demnach korrekt.

Erwähnenswert hierbei ist, daß die Besteuerung von Veräußerungsgewinnen zumindest theoretisch die Versuchung entstehen $1 a ̈ B t$, gegen den Sinn bestehender Gesetze zu verstoßen. Diese versuchung wird offenbar, wenn man sich die steuerlichen Konsequenzen der folgenden Vereinbarung $z$ wischen Käufer und Verkäufer vor Augen führt: beide kommen darin überein, den offiziellen Verkaufspreis $A D$ um eine D-Mark zu reduzieren, wobei diese DMark nun aber nicht dem Käufer zugutekommt, sondern unter der Hand und am Finanzamt vorbei dem Verkăufer zuflieBt. Die einkommensteuerlichen Konsequenzen dieser Verejnbarung sind die folgenden: zunachst sinken unmittelbar die steuerverpflichtungen des verkăufers, da dessen realisierter veräußerungsgewinn vor den Augen des Fiskus' um eben diese D-Mark abgenommen hat. Sinken werden auch die abschreibungsfähigen Anschaffungskosten des Kăufers, und zwar um denselben Betrag. Da diese D-Mark aber nur pro rata temporis hătte abgeschrieben werden dürfen, ist die Vermögenseinbuße, die der Kăufer infolge dieser vereinbarung erleidet, geringer als der vermögenszuwachs des verkäufers. Der Bautrăger könnte den Käufer folglich für die diesem entstandene vermögenseinbuße entschädigen und einen zusatzlichen Rabatt auf den 
inoffiziellen verkaufpreis AD gewähren, ohne daß sich dadurch seine eigene Vermögensposition verschlechtern würde. Auf diese Weise ließe sich der Rentabilitätsnachteil des Erwerbs von einem $z u$ Versteuerung von Veräußerungsgewinnen verpflichteten Bauträger gegenüber dem Erwerb als. Bauherr Schritt für Schritt und Mark um Mark aufholen.

Somit läBt sich unter Rentabilitätsaspekten die folgende Rangfolge von Erwerbsformen aufstellen:

- die vergleichsweise billigste Form des Erwerbs von Wohnungseigentum ist der Kauf von einem Bauherrn, der realisierte Veräußerungsgewinne seines Wohnimmobilienvermögens nicht versteuern muB;

- per saldo kostspieliger ist es, wenn der Wohnungsinvestor Wohnungseigentum in der Funktion des Bauherrn auf eigenen Namen und eigene Rechnung erstellen läßt;

- als die relativ teuerste Form des Erwerbs von Wohnungseigentum entpuppt sich der Kauf der Immobilie von einem Bauherrn, der Veräußerungsgewinne versteuern muß.

Der Vorwurf, daß der Bauherr einer Wohnimmobilie im Vergleich zum Käufer eines identischen Objekts durch das geltende Einkommensteuerrecht bevorzugt behandelt wird und es ihm deshalb eher möglich ist, Wohnimmobilienvermögen $\mathrm{zu}$ bilden, ist demnach zu pauschal. Bei dieser Beurteilung wird übersehen, daß auch die gekaufte Wohnung einen Bauherrn hat, der in den Genuß genau derselben einkommensteuerlichen "Vergünstigungen" gelangt wie jener Bauherr, der selbst als Produzent von Wohnleistungen tätig werden will.

Unsere Uberlegungen zur Einkommensbesteuerung in der Erwerbsphase wären jedoch unvollständig, wenn nicht auf eine weitere Schwäche des jetzigen Steuersystems hingewiesen würde; diese Schwäche ändert zwar nichts an der eben beschriebenen Rangfolge der Erwerbsformen (denn sie kann im Rahmen der beiden erstgenannten Erwerbsformen gleichermaßen verbilligend wirken), sie zeigt aber Wirkungen, die vor allem im Hinblick auf die inter- und intrasektorale Effizienz 
der Einkommensbesteuerung $z u$ einigen kritischen Anmerkungen AnlaB geben.

Bisher wurde unterstellt, dâs alle Leistungen, die zur Bebauung eines Grundstücks erforderlich sind, auf entsprechenden Märkten gehandelt und auf diesem Wege pekuniarisiert werden und deshalb auch einer Bewertung zugänglich sind. Dies wird aber dann nicht mehr der Fall sein, wenn wir es mit einem Bauherrn zu tun haben, der mit Hilfe von Eigenleistungen zum Erwerb des baureifen Grundstücks und zur Errichtung des Gebäudes beiträgt. Die Folge dieser Nichtpekuniarisierung von Bauherrenaufwendungen ist, daß die steuerlich relevanten Gesamtkosten des objekts geringer sind als bei einer Wohnung, deren Bauherr keine Eigenleistungen exbrachte. Vor allem die ausgewiesenen Herstellungskosten werden beim selbst zupackenden Bauherrn geringer ausfallen.

Obwohl also die bei der Errichtung des Wohnungseigentums erbrachten Eigenleistungen zum größten Teil reale Vermögenswerte darstellen, können für sie - unmittelbare Folge der Nichtpekuniarisierung - keire Absetzungen für Abnutzung steuerlich geltend gemacht werden; auch ein sofortiger Abzug als Werbungskosten ist aus demselben Grund nicht möglich. Insofern liegt es nahe, auf eine einkommensteuerliche Benachteiligung des Eigenleistungen erbringenden Bauherrn $\mathrm{zu}$ schließen.

Hierbei würde aber übersehen werden, daß die Eigenleistungen des Bauherrn zugleich auch Leistungsentgelte darstellen, die infolge der Nichtpekuniarisierung von der Einkommensteuer nicht erfaßt werden können. Berücksichtigt man diesen Aspekt, so läßt sich zeigen, daß das geltende Einkommensteuerrecht die Aufnahme von "Muskelhypotheker" attraktiv macht:

- bezüglich der Nichtabzugsfähigkeit nichtpekuniarisierter Werbungskosten ist festzuhalten, daß dem Eigenleistungen erbringenden Bauherrn hieraus weder Vor- noch Nachteile erwachsen. Diese Aussage wird verständiich, wenn man bedenkt, daß sich die Einkommenssteuerzahllast des Bauherrn nicht verändern 
wïrde, wenn es gelänge, die Werbungskosten darstellenden Eigenleistungen $\mathrm{zu}$ bewerten und der Besteuerung zuzuführen. Zwar käme der Bauherr über den Werbungskostenabzug dann in den Genuß einer Einkommensteuerminderung, andererseits müßte er aber dieselben Werbungskosten als Entgelte für erbrachte Leistungen seinem $z u$ versteuernden Einkommen zurechnen - die zusätzliche Steuerverpflichtung entspräche exakt der erzielbaren steuerersparnis;

- dagegen erweist sich die Nichtpekuntarisierung von Herstellungsaufwand darstellenden Eigenleistungen für den Bauherrn als eindeutiger Vorteil: könnte man diesen nämlich bewerten und als Leistungsentgelt der Einkommensbesteuerung unterwerfen, entstünde dem Bauherrn zunächst eine zusätzliche steuerlast. Andererseits könnte er die bewerteten Eigenleistungen in gleicher Höhe im Rahmen der steuerlichen AfA auch steuermindernd geltend machen, jetzt allerdings nicht in Form einer sofortabschreibung, sondern in jährlichen Beträgen über die Nutzungsdauer der Immobilie hinweg. Der Barwert der Steuererstattungen wäre deshalb geringer als der Barwert der zusätzlichen steuerverpflichtungen.

Dieser Vorteil der Nichtpekuniarisierung zeigt sich auch dann, wenn der Bauherr seine zumindest teilweise selbsterrichteten vier Wände nicht weiternutzen, sondern veräußern möchte. In diesem Fall schlagen sich - vollkommene Märkte unterstellt - die monetären Äquivalente seiner Eigenleistungen in (im allgemeinen) steuerfreien Veräußerungsgewinnen nieder.

Zweifellos wird durch die Steuerfreiheit der Eigenproduktion der Anreiz, Eigenleistungen zu erbringen, verstärkt. Die Wohlfahrtseinbußen, die mit dieser steuerfreiheit verbunden sind, zeigen sich deshalb auch nicht nur darin, daß sie die oben aufgezeigten Rentabjlitätsunterschiede in den Erwerbsformen von Wohnungseigentum verstärken. Wohlfahrtsverluste kommen auch dadurch zustande, daß die für die Eigenproduktion benötigte zeit entweder $\mathrm{zu}$ Lasten der von der Einkommenrbesteuerung in vollem Umfang erfaBten Arbeitszeit oder durch Verzicht auf Freizeit aufgebracht wird. 
Verzichtet der Bauherr auf Freizeit, so stört die Erhebung einer Einkommensteuer bundesdeutschen Rechts die ArbeitszeitFreizeit-Entscheidung des privaten Haushalts - und zwar über das der allgemeinen Einkommensteuer ohnehin immanente Maß hinaus und führt auf diesem Wege zu (zusätzlichen) Wohlfahrtseinbußen.

Wird die für die Eigenproduktion benötigte zeit zulasten der arbeitsvertraglichen Arbeitszeit aufgebracht, so gehen in aller Regel die mit dem arbeitsteiligen Produktionsproze $\beta$ verbundenen positiven Wohlfahrtseffekte der Spezialisierung verloren ${ }^{74}$, es sei denn, der Bauherr ist z.B. Bauhandwerker und kann qualifizierte Arbeiten selbst durchführen.

Wenn der Gesetzgeber solche Wohlfahrtsverluste vermeiden will, kommt er nicht umhin, auch die Eigenleistungen des Bauherrn im Rahmen der Einkommensbesteuerung zu erfassen. Ein solches Verfahren wird zwangsläufig dazu führen, daß auch ein Teil der "Freizeit" besteuert wird. Dies ist im wesentlichen auch der Grund dafür, daB die Besteuerung der Haushaltsproduktion von vielen Autoren mit dem Hinweis auf den Bedürfnisbefriedigungscharakter der Freizeit abgelehnt wird ${ }^{75}$ ) Dieses Argument geht jedoch am Kern des Problems vorbei. Kein vernünftiger Mensch käme schließlich auf den Gedanken zu fordern, daß sich die Besteuerung des am Markt erzielten Einkommens daran orientieren müsse, welche Motive der Berufstätigkeit zugrundeliegen, ob Arbeit etwa nur als notwendiges Ubel. zur Sicherung der materiellen Existenz empfunden wird oder nicht auch auf irgend eine weise etwa indem sie einen Beitrag zur Selbstverwirklichung des Arbeitenden leistet - der Bedürfnisbefriedigung des Steuerpflichtigen dient und deshalb konsequenterweise als steuerfreie Freizeitbeschäftigung interpretiert werden muß. Es ist nicht sinn und Zweck der Einkommensbesteuerung, die Steuerfreiheit einer wie auch immer definierten Freizeit zu garantieren, vielmehr ist es

74) STREIT (1982), S.5.

75) Vgl. hierzu z.B. HALLER(1981) oder ANDEL(1980), S.341. 
das Ziel der Einkommensteuer, die Leistungsfähigkeit des Steuerpflichtigen $z u$ erfassen. Und will man den Einkommensbegriff des Reinvermögenzugangs als Leistungsfähigkeitsindikator nicht aufgeben, so ist die einkommensteuerliche Erfassung der bauherrlichen Eigenleistungen geboten. ${ }^{76)}$

Daran ändert auch die Tatsache nichts, daß dem Wohnungseigentum damit im Vergleich $\mathrm{zu}$ anderen in der Freizeit produzierten Gütern und Diensten eine steuerliche Sonderstellung zugewiesen wird. Denn bei fast allen Formen der Haushaltsproduktion erschwert der Umstand, daß die Produzenten ihre Faktorerträge unmittelbar selbst konsumieren, die Erfassung und Bewertung solcher Leistungen erheblich; auch aus allokationstheoretischer sicht erscheint es in diesen Fällen ratsam, auf die steuerliche Erfassung der Haushaltsproduktion angesichts des damit einhergehenden Aufwandes ganz zu verzichten.

Bei der Bewertung von Eigenleistungen im Rahmen der Errichtung von Wohnraum werden sich keine derartigen Hindernisse auftürmen: die einzige Voraussetzung für die Erfassung des mit der Erbringung von Eigenleistungen verbundenen Reinvermögenszugangs wäre ein zeitnahes Verfahren zur Bewertung von Grundstücken. Durch Subtraktion der pekuniarisierten Aufwendungen des Bauherrn von diesem aktuellen Ertragswert erhielte man als Differenz den durch die Eigenleistungen entstandenen Reinvermögenszugang, der nun in voller Höhe der Einkommensbesteuerung zuzuführen wäre.

Gelänge es, ein solch zeitnahes Bewertungsverfahren zu installieren, hätte man sich in eleganter weise auch des zuvor beschriebenen Problems der unterschiedlich rentablen Erwerbsformen entledigt: unabhängig davon, ob der Wohnungsinvestor selbst als Bauherr tätig wird oder sej.n Wohneigentum von einem

76) HALLER (1981), S.47. 
anderen Bauherrn käuflich erwirbt - die Kosten des Erwerbs wären immer dieselben. Beide Bauherrn hätten Aufwendungen in Höhe von $A D$, würden dafür aber durch künftige Mieterträge bzw. durch einen gleichhohen Verkaufspreis in vollem Umfang entschädigt werden. Und auch der Reinvermögenszugang des Käufers wäre gleich Null, da dessen gesammter Bruttovermögensabgang in Gestalt der Anschaffungskosten durch den Marktwert des Grundstücks aufgewogen werden würde.

Die Besteuerung von Eigenleistungen in der eben beschriebenen Art und Weise wäre nicht nur unter wohlfahrtstheoretischen Gesichtspunkten zu begrüßen, sie wäre auch im Sinne unseres Einkommensteuerrechts nur konsequent. Denn während der Gesetzgeber die beim Bau der Wohnung erbrachten Eigenleistungen quasi als Kosten der Lebensführung und deshalb als einkommensteuerlich irrelevant betrachtet, besteuert er die aus diesen Leistungen fließenden Erträge während der Nutzungsphase des Grundstücks. Den Problemen, die in diesem Zusammenhang entstehen, wollen wir uns in folgenden zuwenden.

\subsubsection{Die Einkommensbesteuerung in der Nutzungsphase}

Es sind vor allem zwei Konstruktionsfehler des bundesdeutschen Einkommensteuersystems, die als mögliche Ursachen für durch die Besteuerung in der Nutzungsphase ausgelösten Wohlfahrtsverluste in Frage kommen: zum einen ist dies die Tatsache, daB die Abgrenzung des steuerpflichtigen Einkommens bei allen betrachteten Einkunftsarten erheblich von dem in (III.86) beschriebenen Ideal abweicht, zum anderen der Umstand, daß die Ermittlung des $\mathrm{zu}$ versteuernden Einkommens von Einkunftsart zu Einkunftsart unterschiedlich vorgenommen wird. Dabei beschränkt sich der zuletztgenannte Aspekt nicht allein auf Unterschiede zwischen den Einkunftsarten "Einkünfte aus Gewerbebetrieb" und "Einkünfte aus Vermietung und Verpachtung", auch im Rahmen der "Einkünfte aus Vermietung und Verpachtung" selbst wird das steuerpflichtige Einkommen mit Hilfe zweier sehr ungleicher Verfahren ermittelt - mit Hilfe 
- des Normalverfahrens nach \$21 EStG oder

- des Pauschalierungsverfahrens nach §21a EStG.

Welches dieser Verfahren zur Anwendung gelangt, hängt im wesentlichen davon $a b$, ob der nichtgewerbliche Wohnungsinvestor sein Wohnungseigentum (vollständig) selbst nutzt oder zumindest Teile davon vermietet.

5.2.2.2.1. Der wohnungsinvestor als nicht-gewerblicher Vermieter Die Ermittlung der Einkünfte aus Vermietung und Verpachtung erfolgt in diesem Fall nach einer in anderen Sektoren üblichen Methode - der Summe aller Einnahmen wird die Summe aller Unkosten (Werbungskosten) gegenübergestellt. Die sich hieraus errechnende Differenz - der Uberschuß bzw. der Verlust aus Vermietung und Verpachtung - wird den steuerpflichtigen Einkünften des Eignerhaushalts zugerechnet und erhöht bzw. vermindert auf diesem Wege dessen zu versteuerndes Einkommen.

Angesichts dieser konzeptionellen Ausgestaltung spräche nichts dagegen, der Bestimmung der individuellen Einkommensteuerzah1last die auf der Basis der Reinvermögenszugangskonzeption des Einkommens abgeleitete Steuerbemessungsgrundlage

(III. 86)

$$
\left.\tau_{e}^{W}=\tau_{e}^{(m H}+\dot{P}_{H}^{H}-P_{H} \alpha \delta H-r D\right)
$$

zugrundezulegen. Das geltende Einkommensteuerrecht enthält jedoch eine Reihe von Regelungen, die ein solches Vorgehen für unsere Analyse als nicht ratsam erscheinen lassen.

Unstrittig ist, daß die Bruttomieteinnahmen $\mathrm{mH}$ einer Periode bei der Einkunftsermittlung im Rahmen der Uberschußrechnung in voller Höhe $z u$ berücksichtigen sind.

Auch hat der Gesetzgeber in \$9, Absatz 1, Ziffer 1 EStG bestimmt, daß die mit den Mieterträgen in einem unmittelbaren Zusammenhang stehenden Schuldzinsen zu den abzugsfähigen Werbungskosten zählen. 
Der entscheidende Unterschied zum Ideal aus (III.86) zeigt sich in der Erfassung der Ertragswertänderung $\left(\dot{\mathrm{P}}_{\mathrm{H}} \mathrm{H}-\mathrm{P}_{\mathrm{H}} \alpha \delta \mathrm{H}\right)$ des Grundstückvermögens. Das deutsche Einkommensteuerrecht erlaubt nämlich keine Abschreibung pro rata temporis nach dem Ertragswertverfahren; ebensowenig werden im Rahmen der Einkünfte aus Vermietung und Verpachtung (Ertrags-)Wertzuwächse besteuert weder zum Zeitpunkt ihrer Realisierung noch zum Zeitpunkt ihrer Entstehung.

Als "Abschreibungen" steuermindernd geltend gemacht werden dürfen laut Einkommensteuergesetz lediglich Kosten, die mit der abnutzungsbedingten Substanzminderung des Gebäudekapitalbestandes im Zusammenhang stehen. Für den Grund und Boden dürfen keine Absetzungen für Abnutzung angesetzt werden - "weil der Grund und Boden keiner Abnutzung unterliegt." 77)

Bemessungsgrundlage für die steuerliche Absetzung wäre demnach - sofern keine welteren Einschränkungen bestünden - der (kalkulatorische) Gebäudekapitalstock $k$, für dessen zeitliche Entwicklung wir in Abschnitt III.2.1.2. die Bewegungsgleichung

(III. 38) $\quad \dot{K}=F_{E}^{d}-(\delta K-E)$

abgeleitet haben. Danach entspricht die zeitliche Veränderung des Gebäudekapitalstocks $z u$ einem beliebigen zeitpunkt dem Saldo aus in Neubauten investiertem und im zuge von Renovierungsarbeiten reinvestiertem Gebäudekapital $\left(F^{d} \varepsilon+E\right)$ einerseits und der via Abnutzung eingetretenen Substanzminderung des gesamten Kapitalstocks andererseits.

Nun wird in (III.38) aber unterstellt, daß die Finanzbehörden den tatsächlichen Werteverzehr des Gebäudekapitals kennen und diesen der Festsetzung der steuerlichen AfA auch zugrunde-

77) SCHÖNHOFER/REINISCH, Gruppe 15, S.64. Daß diese Begründung nicht von ökonomischer Denkart zeugt, muB vor dem Hintergrund unserer Analyse aus Abschnitt III.5.1. nicht besonders betont werden. 
legen. Schlagworte wie "degressive AfA" oder "erhöhte AfA" weisen jedoch deutlich darauf hin, daß dem in der Praxis nicht so ist.

Zusätzliche Verwirrung stiftet hierbei die Tatsache, daß das Einkommensteuergesetz dem Wohnungsinvestor dret verschiedene Abschreibungsverfahren zur (allerdings nicht völlig freien) Auswahl stellt;im einzelnen wären dies

- die lineare Absetzung für Abnutzung (\$7, Absatz 4 EStG): die Grundform der Absetzung für Abnutzung ist die gleichmässige Verteilung der Anschaffungs- bzw. Herstellungskosten des Gebäudes auf die Nutzungsdauer des Grundstücks; bei Gebäuden, die nach dem 31.12. 1974 fertiggestellt wurden, können nach Maßgabe der linearen AfA 2 Prozent der Anschaffungs- bzw. Herstellungskosten in jeder Steuerperiode solange steuermindernd geltend gemacht werden, bis das Gebäude zu hundert Prozent abgeschrieben ist; dem entspricht eine steuerliche Nutzungsdauer von 50 Jahren ${ }^{78}$ ) Für unsere späteren tuberlelegungen wird von Bedeutung sein, daß im Falle eines Verkaufs die beim Verkäufer verstrichene Nutzungsdauer nicht zählt: auch der Käufer kann die von ihm getragenen Anschaffungskosten zu 100 Prozent abschreiben, unabhängig von der Höhe der Summe der vom bisherigen Eigentümer vorgenommenen Abschreibungen.

- die degressive Absetzung für Abnutzung ( $\$ 7$, Absatz 5 EStG): die Möglichkeit, Gebäude degressiv abschreiben zu können, ist seit 1965 gegeben. Sie war seinerzeit ausschließlich dem Wohnungsinvestor gestattet, der sein wohnungseigentum in der Funktion des Bauherrn erworben hatte. Seit November 1979 wird sie auch den Käufern von Wohnimmobilien eingeräumt - soweit zwei Bedingungen erfüllt sind:

- die Anschaffung muß bis zum Ende des Jahres der Fertigstellung des Gebäudes erfolgen und

- der VeräuBerer darf für sich weder degressive noch erhöhte Absetzungen oder Sonderabschreibungen in Anspruch genommen haben.

78) Bei Gebäuden, die vor dem 31.12.1974 fertiggestellt worden sind, sind jährlich 2,5 Prozent der Anschaffungs- bzw. Herstellungskosten absetzbar. 
Nach neustem Recht vermindert sich durch die degressive AfA das $\mathrm{zu}$ versteuernde Einkommen des Wohnungsinvestors

- im Jahr der Fertigstellung oder Anschaffung und in den darauf folgenden sieben Jahren um jeweils 5 Prozent

- in darauffolgenden sechs Jahren um jeweils 2,5 Prozent und - in den darauffolgenden sechsunddreissig Jahren um jeweils 1,5 Prozent

der Herstellungs- oder Anschaffungskosten. 79)

Auch bei der degressiven AfA wird also stets eine Nutzungsdauer von 50 Jahren zugrundegelegt.

- die erhöhte Absetzung für Abnutzung ( $\$ 7 \mathrm{~b}$ EStG): ausschlaggebend für die Einführung der erhöhten Absetzung in das Einkommensteuerrecht war die Absicht des Gesetzgebers, in der Nachkriegszeit steuerliche Anreize für die Schaffung neuen Wohnraums zu geben; die Vergünstigungen des $\S 7 \mathrm{~b}$ EStG waren deshalb zunächst auf Neubauten beschränkt. Nach vielfachen Änderungen wurden mit Wirkung von 1977 an alle Anschaffungen von Einfamilienhäusern, Zweifamilienhäusern und Eigentumswohnungen in die 7b-Regelung miteinbezogen, unabhängig davon, ob es sich bei dem betreffenden Gebäude um einen Neubau oder einen Altbau handelt, wie oft die Immobilie zuvor den Eigentümer gewechselt hat und in weIcher Höhe die vorherigen Eigentümer für sich Absetzungen geltend gemacht

79) Diese gegenüber früher erhöhten Abschreibungssätze gelten ausschließlich für Gebäude, für die der Antrag auf Baugenehmigung nach dem 29.7.1981 gestellt oder mit deren Errichtung erst nach dem 29.7.1981 begonnen worden ist oder die erst nach dem 29.7.1981 gekauft worden sind (BFH, Urteil vam 2.2.1982, BStBl 1982II, S.390). Für Gebäude, die diese Bedingungen nicht erfüllen, gilt die alte Regelung, nach der in den ersten zwölf Jahren der Nutzung jeweils 3,5 Prozent, in den darauffolgenden 20 Jahren jeweils 2 Prozent und in den hierauf folgenden 18 Jahren jeweils 1 Prozent der Anschaffungs- bzw. Herstellungskosten absetzbar sind. Erwähnenswert am Rande ist auch, daß die "degressive" AfA anders als die lineare AfA bereits im Jahr der Fertigstellung bzw. Anschaffung mit dem vollen Jahresbetrag abgezogen werden kann, selbst dann, wenn das Gebäude erst kurz vor Jahresende fertiggestellt oder angeschafft wird. 
haben.

Daß ein solch umfassender Anwenciungstereich dazu einlädt, die Segnungen des $\S 7 \mathrm{~b}$ EStG intensiv zu nutzen, bestätigt die Erfahrung. Um $z u$ verhindern, daß Grundstücksübereignungen ausschließlich zur Ausnutzung der erhöhten Absetzungen vorgenommen werden, hat der Gesetzgeber zum einen bestimmte Transaktionen von der Inanspruchnahme der 7b-Abschreibung ausgeschlossen (wie z.B. den Kauf vom nicht-getrennt lebenden Ehegatten oder den Rückerwerb) und zum anderen die sogenannte objektbeschränkung formuliert, nach deren Maßgabe ein Steuerpflichtiger erhöhte AfA nur einmal in seinem Leben in Anspruch nehmen kann.

Als erhöhte Absetzungen kann der Wohnungsinvestor

- im Jahr der Herstellung oder Anschaffung und in den sieben folgenden Jahren jeweils bis zu 5 Prozent der (begrenzten) Anschaffungs- oder Herstellungskosten geltend machen.

- der Restbuchwert kann in den vierzig fol-genden Jahren 1inear zu jeweils 2,5 Prozent abgeschrieben werden.

Die steuerliche Nutzungsdauer beträgt im Falle der erhöhten Absetzungen demnach nur 48 Jahre. Ebenfalls im Gegensatz zu linearer und degressiver AfA steht die Tatsache, daB für den nach $\S 7$ b EStG ansetzbaren Gebäudewert genau festgelegte obergrenzen gelten: bei Einfamilienhäusern dürfen mit wirkung des Zweiten Haushaltsstrukturgesetzes vom 22.12.1981 höchstens 200000 D-Mark (zuvor 150000 D-Mark) und bei Zweifamilienhäusern maximal 250000 D-Mark (zuvor 200000 D-Mark) erhöht abgeschrieben werden. Für den Differenzbetrag zwischen den tatsächlichen und den zulässigen Anschaffungs- bzw. Herstellungskosten gelten die Bestimmungen des $\S 7$, Absatz 4 EStG.

Dieses Nebeneinander von drei verschiedenen Abschreibungsverfarren ist nicht nur veruirrend, es scheint unter Rentabilitätsaspekten auch überflüssig $z u$ sein. Denn es ist offensichtlich, daß sich ein Wohnungsinvestor, der seine Einkünfte aus Vermietung und Verpachtung im Rahmen der Uberschußrechnung ermittelt, bei der Inanspruchnahme der degressiven AfA seit dem Zweiten 
Haushaltsstrukturgesetz - sieht man von cier Möglichkeit zum Vortrag der 7b-Abschreibung auf der Lohnsteuerkarte einmal ab immer besser stellt als bei der Inanspruchnahme der 7b-Option (und erst Recht der Inanspruchnahme der Option auf lineare Aff.). Während die degressiven und die erhöhten Abschreibungssätze in den ersten acht Jahren der Nutzungsphase übereinstimmen, erweist sich die degressive AfA in den darauffolgenden Jahren schon deshalb als vorteilhafter, weil im Rahmen der erhöhten AfA nach Ablauf des achtjährigen Begünstigungszeitraums jährlich nur noch 2,5 prozent des bilanziellen Restwertes abgesetzt werden dürfen (dies entspricht 1,5 prozent der ursprünglichen Anschaffungs- bzw. Herstellungskosten), im Rahmen der degressiven AfA dagegen 2,5 Prozent der ursprünglichen Anschaffungsbzw. Herstellungskosten. Hinzu kommt, daB bei der degressiven AfA keine Abschreibungsobergrenzen $\mathrm{zu}$ beachten sind. $\left.{ }^{80}\right), 81$ )

So unterschiedlich die Rentabilitätseffekte der drei steuerlich zulässigen Abschreibungsverfahren auch sein mögen - eine Eigenschaft ist ihnen allen gemeinsam: während man bei der Berechnung der Kostenmieten im sozialen Wohnungsbau von einer jährlich einprozentigen Wertminderung des Gebäudekapitals und mithin von einer ökonomischen Lebensdauer des Gebäudes von

80) Dieses Urteil gilt strenggenommen nur für den speziellen Fall eines proportionalen Einkammensteuertarifs. Bei einer Einkanmensteuer mit progressivem 'Tarifverlauf besitzt es u.U. dann keine Gultigkeit mehr, wenn das Haushaltseinkommen im Zeitablauf zunimmt; vgl. dazu auch HEUER (1982)

81) Die von der Zahl der eigenen, zum Haushalt gehörenden Kinder abhängige Steuerermäßigung des $\$ 34$ f EStG - das sogenannte "Baukindergeld"-, das an die Inanspruchnahme der 7b-Abschreibung gekoppelt ist, ist für den der Überschußrechnung unterliegenden Wohnungsinvestor irrelevant. 
100 Jahren ausgeht, unterstellen alle Abschreibungsverfahren eine wesentlich kürzere Nutzungsdauer. Das Steuergesetz erlaubt also in jedem der drei betrachteten Fälle, daß das Gebäudekapital "schneller" abgeschrieben werden darf, als dies der tatsächlichen abnutzungsbedingten Substanzminderung dieses Kapitals entspricht.

Da sich unsere Analyse nicht die Quantifizierung der mit den verschiedenen steuerlichen Regelungen einhergehenden Wohlfahrtsverluste zum ziel gesetzt hat, sondern sich damit begnügt, die Existenz solcher Verluste nachzuweisen, reicht es für die Zwecke unserer Untersuchungen völlig aus, wenn wir alle drei Abschreibungsvarianten durch ein einziges fiktives Abschreibungsverfahren hinreichend genau approximieren. Die Bedingung, die dieses fiktive Verfahren erfüllen muß, ist die, daß unter seinem Regime der bilanzielle Kapitalstock $\mathrm{K}^{*}$ schneller an Substanz verliert als der kalkulatorische Kapitalstock K. Offensichtlich erfüllt ein geometrisch degressives Abschreibungsverfahren, dessen Abschreibungssatz $\mu$ größer ist als die ökonomisch korrekte Abnutzungsrate $\delta$, diese Bedingung ohne Einschränkung.

Die Lücke, die sich als unmittelbare Folge der aktuellen steuerlichen Regelungen zwischen dem kalkulatorischen und dem bilanziellen Kapitalstock auftut, wird zusätzlich noch dadurch vergrößert, daß Erhaltungsaufwand im Gegensatz zum Modernisierungsaufwand in der steuerbilanz nicht aktiviert wird, sondern zum Zeitpunkt seines Entstehens als Werbungskosten von der Einkommensteuerbemessungsgrundlage abgezogen werden kann $(\S 11, \mathrm{Ab}-$ satz 2 EStG).

Unter Beachtung dieser institutionellen Regelungen läßt sich die zeitliche Veränderung des bilanziellen Kapitalstocks deshalb mit Hilfe der Bewegungsgleichung

$$
\dot{K}^{*}=F_{\varepsilon}-\mu K^{*}
$$

beschreiben. 
Das zu versteuernde Einkommen des Wohnungsinvestors erhalten wir, wenn wir den gesetzlichen Bestimmungen folgend von den Bruttomieteinnahmen $\mathrm{mH}$ die Ausgaben für Erhaltungsaufwand $\mathrm{E}$ sowie die effektiv gezahlten Fremdkapitalzinsen rD und die bilanzielle Abschreibung $\mu K^{*}$ subtrahieren. Entsprechend yilt für die Einkommensteuerzahllast des Wohnungsinvestors die Beziehung

(III.120)

$$
T_{e}^{W}=\tau_{e}\left(m H-E-r D-\mu K^{*}\right) .
$$

Unter Beachtung von (III.120) verbleibt nach Abzug der zu zahlenden Einkommensteuer ein für Entnahmezwecke zur Verfügung stehender D-Mark-Betrag in Höhe von

$$
\begin{aligned}
A_{W}= & z \theta_{e}(m H-E-r D)+s-F^{d} \varepsilon-P_{B} F^{d}+z \tau_{e} \mu K^{*} \\
& +\left[\gamma_{2} \rho_{1}+\left(1-\gamma_{2}\right) \rho_{2}\right] \tau_{e}\left(F^{d} \varepsilon+P_{B} F^{d}\right)
\end{aligned}
$$

Der Term in der zweiten Zeile von (III.121) soll den in Abschnitt. III.5.2.2.1. beschriebenen Kostenunterschieden der diskutierten Erwerbsformen Rechnung tragen. Die Indikatorvariablen $\rho_{1}$ und $\rho_{2}$ sollen hierbei die Rentabilitätsrangfolge dieser Erwerbsformen widerspieqeln; es gilt $0 \leq \rho_{1} \leq \rho_{2} \leq 1$. Wie wir gesehen haben, entspricht allein der an dritter stelle der Rangfolge stehende Erwerb von einem zur Versteuerung realisierter Wertzuwächse verpflichteten Bauherrn den Anforderungen einer effizienten Form der Einkommensbesteuerung: infolge der Immobilientransaktion ändert sich auch unter dem Regime des geltenden Einkommensteuerrechts weder beim Verkäufer (Gesamtkosten = Verkaufspreis) noch beim Käufer (Marktwert = Kaufpreis) das Reinvermögen; es kommt in beiden Fällen lediglich zu einer Umschichtung von Vermögensbeständen, die für die Bemessung der Einkommensteuerzahllast irrelevant ist. 
Nicht mehr mit den Erfordernissen einer effizienten Besteuerung in Einklang zu bringen ist der Fall, in dem der Wohnungsinvestor Immobilienvermögen in seiner Funktion als Bauherr erworben hat. Er gelangt auf dem Weg der Werbungskostenerstattung, deren ökonomische Konsequenzen mit den $\mathrm{Z}$ insvorteilen einer Sofortabschreibung vergleichbar sind, in den Genuß eines realen Vermögenszugangs, der aber steuerfrei bleibt.

Dieser ausschließlich steuerbedingte Reinvermögenszugang ist noch größer, der komparative Vorteil der Erwerbsform noch ausgeprägter, wenn der Wohnungsinvestor Wohnimmobilienvermögen von einem Bauherrn käuflich erwirbt, der VeräuBerungsgewinne steuerfrei realisieren kann. Neben dem Werbungskostenabzug, dessen Vorteile der Bauherr an den Käufer weitergibt, kann der Käufer darüberhinaus im Rahmen der steuerlichen AfA einen im Vergleich zum bauherrlichen Wohnungsinvestor höheren Betrag an Erwerbskosten steuermindernd gelten machen.

Offensichtlich weichen die für die Bemessung der AfA relevanten steuerlichen Buchwerte trotz gleicher Gesamtkosten bei allen drei Erwerbsformen voneinander ab. Um Wohnungsinvestitionen unabhängig von der Art ihres Erwerbs in der Nutzungsphase trotzdem miteinander vergleichen zu können, wollen wir unterstellen, daß identische Wohnobjekte auch in der Steuerbilanz als solche behandelt werden. Den mit den beiden billigsten Erwerbsformen verbundenen komparativen Vermögensvorteilen wollen wir in unserer Analyse durch die Unterstellung Rechnung tragen, daß der Staat diese Erwerbsformen mittels offener Transfers unterschiedlich stark subventioniert; die Höhe der Transfers verhalte sich dabei proportional zu den Gesamtkosten $F_{\varepsilon}{ }^{d}, P_{B} F^{d}$ der Immobilie. Dementsprechend indiziert die Parameterkonstellation

- $\rho_{1}=\rho_{2}=0$ die relativ teuerste, aber im Sinne des Wohlfahrtsoptimums effiziente Erwerbsform; 
$-r_{1}=1, p_{2}>\rho_{1}>0$ den Fall, in dem der vermietende Wohnungsinvestor Wohnungseigentum als Bauherr erworben hat;

$-r_{1}=0, \rho_{2}>\rho_{1}>0$ die situation, in der die Wohnimmobilie von einem Bauherrn veräuBert wurde, der realisierte Wertzuwächse nicht versteuern muB.

Ziel des Wohnungsinvestors muB es nun wieder sein, die Summe der Barwerte der aktuellen sowie aller zukünftigen Entnahmen zu maximieren. In formaler Hinsicht ist dabei zu beachten, daß mit dem bilanziellen Kapitalstock $\mathrm{K}^{*}$ eine weitere Zustandsvarlable in das Planungsproblem des repräsentativen Wohnungs-“ investors Eingang gefunden hat. Dieses lautet nun

(III.122)

$$
\{S, E, \varepsilon, F\} \quad M_{W}=\int_{t_{0}}^{\infty} A(t) \exp [-z \theta e r(t-t *)] d t,
$$

unter den Bedingungen (II.2), (II.3), (II.9), (II.10), (II.14), (II.16), (II.17a), (III.119) und (III.121).

Die Hamiltonfunktion für das Kontrollproblem aus (III.122) lautet entsprechend in laufenden werten

$$
\begin{aligned}
H= & z \theta_{e}(m H-E-r D)+S+z \tau_{e} e^{\mu K^{*}} \\
& -\left[1-\left(\gamma_{2} \rho_{1}+\left(1-\gamma_{2}\right) \rho_{2}\right] \tau_{e}\left(F^{d} \varepsilon+P_{B} F^{d}\right)\right. \\
& +P_{H}\left[F^{d} \varphi(\varepsilon)-z(\alpha \delta H-E)\right] \\
& +\lambda_{K^{*}}\left(F^{d} \varepsilon-z \mu K^{*}\right) \\
& -\lambda_{D} S .
\end{aligned}
$$

$\lambda_{\mathrm{K}^{*}}$ ist der Schattenpreis des bilanziellen Kapitalstocks.

Die erste Frage, auf die wir eine Antwort geben müssen, ist die nach der optimalen Finanzierungsform. Als notwendige Bedingung für den optimalen Finanzierungsweg erhalten wir über die partielle Differentiation von (III.12 3) nach der Kontroll- 
variablen $\mathbf{S}$

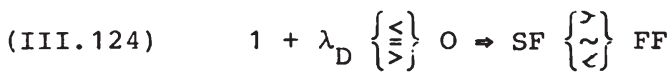

Wegen $\lambda_{\mathrm{D}} \equiv \partial \mathrm{M}_{\mathrm{W}} / \partial \mathrm{D}=-1$ folgt aus (III.12), daß der vermietende wohnungsinvestor weder der Fremdfinanzierung noch der selbstfinanzierung den vorzug gibt. Der Grund für diese Finanzierungsindifferenz ist zum einen in der Tatsache zu sehen, daß auch das geltende Einkommensteuerrecht im Rahmen der Überschußrechnung den Ansatz von Schuldzinsen als Werbungskosten erlaubt. Dies hat zur Folge, daß sich für jede aufgenommene D-Mark Fremdkapital die effektiven Finanzierungskosten um $\tau e^{r}$ D-Mark auf ${ }_{\mathrm{e}}^{\mathrm{r}} \mathrm{D}$-Mark vermindern. Zum zweiten unterliegen die Erträge alternativer Anlagemedien ebenfalls der Ertragsbesteuerung - so sinkt beispielsweise der effektive Zinsertrag einer am Kapitalmarkt investierten $\mathrm{D}$-Mark steuerbedingt von $\mathrm{r} D$-Mark auf $\Theta_{\mathrm{e}} \mathrm{r}$ D-Mark. Infolgedesssen kann der Wohnungsinvestor aus einer (marginalen) Veränderung der Finanzierungsstruktur seines Wohnflächenbestandes für die Eigner seines Unternehmens keinen zusätzlichen Vermögensvorteil ziehen - Kosten und Erträge dieser Umstrukturierung werden sich zu jedem Zeitpunkt nach Einführung der steuer aus der Sicht des Unternehmenseigners die Waage halten.

Auch auf die Wahl des optimalen Zeitpfades für den Erhaltungsaufwand wird die Einführung einer Einkommensteuer nach \$21 EStG keinen Einfluß haben: da wir unterstellt haben, daß der Häuserpreis $z u$ jedem Zeitpunkt jenseits von $t_{0}$ größer als Eins ist (und deshalb $y=1 \mathrm{gilt}$, wind zu jedem zeitpunkt $t>t_{0}$ der abnutzungsbedingte Substanzverzehr des Gebäudekapitalstocks in vollem umbang durch werterhcltende Maßnahmen kompensiert. Allerdings verursachen werterhaltende Maßnahmen aufgrund geltender einkommensteuerrechtlicher Bestimmungen geringere effektive Kosten als in einer steuerfreien Welt bzw. in einer ökonomie mit scHANzscher Einkommensteuer. Der Fiskus erlaubt nämlich den sofortigen Abzug - die Sofortabschreibung - des Erhaltungsaufwandes als Werbungskosten, was aus ökonomischer sicht aber nicht korrekt ist. Im ökonomischen sinne korrekt 
wäre es, Ausgaben für Renovierungsarbeiten steuerlich genauso zu behandeln wie Ausgaben für Neubauleistungen. Demzufolge müBte Erhaltungsaufwand in der Steuerbilanz aktiviert und nach Maßgabe des relevanten Abschreibungsverfahrens pro rata temporis über die Nutzungsphase hinweg steuerlich abgesetzt werden.

Stimmen Finanzierungs- und Renovierungspläne des vermietenden Wohnungsinvestors unter dem Regime der Ubberschußrechnung geltenden Rechts mit den entsprechenden paretoeffizienten Programmen noch überein, so ist zu vermuten, daß sich die paretoeffiziente Neubauplanung unter dem Einfluß des geltenden Steuerrechts verändert. Um diese Veränderungen beschreiben zu können müssen wir die Hamiltonfunktion aus (III.123) partiell nach den Kontrollvariablen $\varepsilon$ und $F^{d}$ sowie nach der Zustandsvariablen $H$ ableiten. 83 )

$\mathrm{Da}$ aus der Finanzierungsindifferenz des Wohnungsinvestors $\partial H / \partial S=O$ folgt, vereinfachen sich die partiellen Differentiale aus (II.28), (II.29) und (II.30) zu

$$
\begin{aligned}
\frac{\partial H}{\partial \varepsilon}= & -\left[1-\left(\gamma_{2} \rho_{1}+\left(1-\gamma_{2}\right) \rho_{2}\right) \tau_{e}\right] F^{d}+P_{H^{\prime}} F^{d} \varphi^{\prime}(\varepsilon) \\
& +\lambda_{K *} F^{d} .
\end{aligned}
$$

(III. 126)

$$
\begin{aligned}
\frac{\partial H}{\partial F^{d}}= & -\left[1-\left(\gamma_{2} \rho_{1}+\left(1-\gamma_{2}\right) \rho_{2}\right) \tau_{e}\right]\left(\varepsilon+P_{B}\right)+P_{H} \varphi(\varepsilon) \\
& +\lambda_{K *} \varepsilon\left\{\begin{array}{l}
<\} \\
=j
\end{array} P_{B} \Rightarrow F^{d}\left\{\begin{array}{l}
= \\
\geq
\end{array}\right\} 0,\right.
\end{aligned}
$$

$$
\frac{\partial H}{\partial H}=\dot{\mathrm{P}}_{H}-z \Theta_{e} r P_{H}=-z\left(\Theta_{e} m-\alpha \delta P_{H}\right) \text {. }
$$

Bedingung (III.125) verlangt, daB im Gleichgewicht das Wertgrenzprodukt der im Wohnungsneubau eingesetzten Bauleistungen übereinstimmt mit den Kosten, die diese Leistungen verursachen aus (III.125) folgt

(III.125)'

$$
P_{H^{\prime}} \varphi^{\prime}(\varepsilon)=1-\tau_{e}\left(\gamma_{2} \rho_{1}+\left(1-\gamma_{2} j \rho_{2}\right)-\lambda_{K *} \cdot\right.
$$

83) Auf die explizite Darstellung der Ableitung von (III.123) nach $K^{*}$ kann verzichtet werden, da diese uns keine zusätzlichen Informationen zu liefern vermag. 
Wie man der rechten Seite von (III.125)' entnehmen kann, sinken die Bauleistungskosten, die vor Steuer 1 D-Mark pro Leistungseinheit betragen, unter dem Einfluß der Steuer:

- zum einen partizipiert der staat in einem je nach Erwerbsform unterschiedlich hohen Maß unmittelbar an den Gestehungskosten des Gebäudes;

- zum anderen verbilligt die Möglichkeit, das Gebäudekapital degressiv uber die Nutzungsphase des Gebäudes hinweg abschreiben zu können, den Einsatz dieses Produktionsfaktors: $\lambda_{K^{*}}$ steht für den Barwert der steuererstattungen, den die letzte in $t_{0}$ in Bauleistungen investierte $D$-Mark auf dem wege des Werbungskostenabzuges erwirtschaftet; aus der Definition des Schattenpreises folgt nämlich

(III.128)

$$
\lambda_{K^{*}} \equiv \frac{\partial M_{W}}{\partial K^{*}}=\frac{\tau e^{\mu}}{\Theta_{e^{r}+\mu}} .
$$

Es verbilligt sich infolge der Besteuerung aber nicht nur der Einsatz des Produktionsfaktors "Bauleistungen", auch die Kosten des Produktionsfaktors "Bauboden" sinken steuerbedingt und auch hier in Abhängigkeit von der Erwerbsform unterschiedlich stark. In die Bemessungsgrundlage der steuerlichen AfA finden die Anschaffungskosten des baureifen Grundstücks dagegen keinen Eingang. Aus diesem Grunde fällt die steuerinduzierte Kostensenkung beim Produktionsfaktor "Bauboden" auch schwächer aus als beim Produktionsfaktor "Bauleistungen": der Einsatz von Bauland wird bei der Produktion von Wohnraum durch die geltenden Abschreibungsverfahren diskriminiert. Die Folgen dieser Diskriminierung werden offensichtlich, wenn wir (III.126) unter Berücksichtigung von (III.125)' und der in (II.37) und (II.38) beschriebenen Eigenschaften der partiellen Produktionselastizitäten umformen $\mathrm{zu}$

$$
\frac{\beta}{\alpha} \varepsilon\left(1-\frac{\lambda_{K} *}{1-\tau_{e}\left(\gamma_{2} p_{1}+\left(1-\gamma_{2}\right) P_{2}\right)}\right)\left\{\begin{array}{l}
< \\
=
\end{array}\right\} P_{B} \Rightarrow F^{d}\left\{\begin{array}{l}
= \\
\geq
\end{array}\right\} 0 .
$$


Da der Klammerausdruck in (III. 126)' kleiner als Eins ist ${ }^{84)}$, steigt bei unveränderten Bodenpreisen die Kapitalintensität der Bebauung in unmittelbarer Reaktion auf die einkommensteuerliche Präferierung des Produktionsfaktors "Bauleistungen".

Ungeachtet der steuerlichen Ungleichbehandlung der für die Produktion von Wohnflächeneinheiten benötigten Produktionsfaktoren bleiben die paretooptimalen Beziehungen zwischen den Wachstumsraten von Häuserpreis, Kapitalintensität und Bodenpreis unverändert bestehen. Aus (III.125)' folgt

(III. 129)

$$
\hat{\mathrm{P}}_{\mathrm{H}}=\beta \hat{\varepsilon}_{\text {, }}
$$

aus (III.126)' leitet man die Beziehung

$$
\left(\text { III. 130) } \quad \hat{\varepsilon}=\hat{\mathrm{P}}_{\mathrm{B}}\right.
$$

$a b$.

Wie ein Blick auf Bedingung (III.127) zeigt, beeinfluBt die Einkommensteuer geltenden Rechts auch die Nutzungskosten des im Wohnungssektor gebundenen Vermögens, wobei man, beschränkt man sich auf die partialanalytische Betrachtungsweise dieses Abschnitts, a priori nicht sagen kann, ob diese Kosten steuerbedingt fallen oder zunehmen; löst man (III.127) nach $\dot{\mathrm{p}}_{\mathrm{H}}$ auf und dividiert anschlieBend durch $\mathrm{P}_{\mathrm{H}^{\prime}}$, erhält man als notwendige $\mathrm{Be}-$ dingungen für das Portefeuillegleichgewicht

$$
\begin{array}{lll}
\text { (III.131a) } & \hat{\mathrm{p}}_{\mathrm{H}}=0 & \text { für } t_{0} \leq t<t *, \\
\text { (III.131b) } & \hat{\mathrm{p}}_{\mathrm{H}}=-\frac{\theta_{e^{m}}^{m}}{\mathrm{P}_{H}}+\theta_{e^{r}+a \delta} & \text { für } t \geq t * .
\end{array}
$$

84) Im Interesse ökonanisch sinnvoller Ergebnisse wird in (III. 126) ' darüberhinaus $\lambda_{K^{*}}<1-\tau_{e}\left(\gamma_{2} \rho_{1}+\left(1-\gamma_{2}\right) \rho_{2}\right)$ unterstellt. 
Wie (III.131b) zeigt, erhöhen sich - wählt man das Allokationsoptimum als Vergleichsmaßstab - die Nutzungskosten des Vermögens, weil die abnutzungsbedingten wertminderungen des wohnflächenbestandes nicht in die Bemessung der Einkommensteuerzahllast eingehen, obwohl sie ebenso wie die absetzbaren Fremdkapitalzinsen ökonomisch eindeutig als Reinvermögensminderung $z u$ interpretieren sind. Andererseits sinken unter dem EinfluB der Besteuerung diese Nutzungskosten insofern, als die nachfragebedingte wertsteigerung des Wohnflächenbestandes, zweifelsfrei ein Reinvermögenszuwachs, von der bundesdeutschen Einkommensteuer ebenfalls nicht erfaßt wird. Ob das aktuelle, den nicht-gewerblichen Vermieter betreffende Einkommensteuerrecht eine Vermögensanlage in bebauten Grundstücken gegenüber anderen Anlageformen begünstigt oder diskriminiert, hängt offensichtlich von der Entwicklung des Marktwertes des Wohnimmobilienvermögens im Zeitablauf ab - es gilt $\left(\mathrm{P}_{\mathrm{H}}{ }^{\mathrm{H}}\right)=\dot{\mathrm{P}}_{\mathrm{H}} \mathrm{H}-\mathrm{P}_{\mathrm{H}} a \delta \mathrm{H}$ : nimmt der Marktwert im Zeitablauf $\mathrm{zu}(\mathrm{ab})$, entpuppen sich die oben beschriebenen Abweichungen von der Konzeption des Reinvermögenszugangs letztendlich als steuerliche Begünstigung (Diskriminierung) dieser Vermögensart.

Diese Aussagen gelten wohlgemerkt nur dann, wenn man das Ent.scheidungsproblem des Wohnungsinvestors isoliert betrachtet. Denn aus der Analyse des Planungsproblems aus Abschnitt III.5.2.1. ist uns bekannt, daß Wertsteigerungen des Bestandes baureifer Grundstücke ebenfalls nicht steuerpflichtig sind. Insofern kommt der sich aus der Steuerfreiheit des Betrages $\dot{\mathrm{P}}_{\mathrm{H}} \mathrm{H}$ ergebende Rentabilitätsvorteil des Wohnimmobilienvermögens gegenüber der Anlageform "baureife Grundstücke" nicht zum Tragen. Diesen Sachverhalt spiegeln auch unsere Analyseergebnisse wider: wie alis (III.129) und (III.130) in Verbindung init (III.110b) hervorgeht, reagiert der Wohnungsinvestor auf die steuerinduzierte Änderung im Angebotsverhalten des repräsentativen Bodenmaklers in einer Weise, die zu einem verlangsamten Wachstum des Häuserpreises führt; es gilt

(III.132)

$$
\hat{\mathrm{P}}_{\mathrm{H}}=\beta \hat{\mathrm{P}}_{\mathrm{B}}=\beta \Theta_{\mathrm{e}} \mathrm{r} \text {. }
$$


Während die Steuerfreiheit von Wertzuwächsen des Grundvermögens somit keine Auswirkungen hat auf die relative Vorteilhaftigkeit einer Vermögensanlage in Form bebauter Grundstücke, erweist sich die Nichtabzugsfähigkeit abnutzungsbedingter wertminderungen dieses Vermögensbestandes als eine eindeutige Diskriminierung gegenüber allen nicht der Abnutzung unterliegenden Vermögensformen.

Formal schlagen sich die damit einhergehenden Effizienzverluste in einer Erhöhung der Nutzungskosten des Grundstückvermögens, graphisch in einer Rechtsverschiebung der Nachfragekurve nieder; löst man (III.131b) nach $P_{H}$ auf und substituiert für $\hat{\mathrm{P}}_{\mathrm{H}}$ die in (III.132) gefundene Beziehung, so erhält man die Gleichung für die Nachfragekurve $N^{\prime}$ in Abb.15:

$$
P_{H}=\frac{m(a / H)}{(1-\beta) r+\alpha \delta / \theta_{e}} \text {. }
$$

Vergleicht man (III.133) mit der entsprechenden LaisserFaire-Funktion, so erkennt man, daß der Häuserpreis $\mathrm{P}_{H}$ unter dem EinfluB der Steuer und unter sonst gleichen Bedingungen insbesondere bei gegebenem Wohnflächenbestand - sinkt.

Diesen die Nutzungskosten von Wohnimmobilienvermögen erhöhenđen, die Vermögensanlage in Form von Mietwohngrundstücken diskriminierenden Eigenarten des deutschen Einkommensteuerrechts steht die Tatsache entgegen, daß die öffentliche Hand sowohl den Erwerb von Bauland als auch (und in noch stärkerem Maße) die Kosten für die Errichtung des Gebäudes subventioniert und so die Vermögensbildung in Form von Wohnimmobilien vergleichsweise attraktiver macht. Graphisch kommt diese Begünstigung in einer Rechtsverschiebung der Angebotskurve zum Ausdruck. Substituiert man in die allgemeine Gleichung der Angebotsfunktion die Inverse der Optimalitätsbedingung (III.125)', so erhält man für die unter dem Regime der deutschen Einkommensteuer zustandekommende Angebotsfunktion für Wohnleistungseinheiten 
$\mathrm{Abb} \cdot 15$

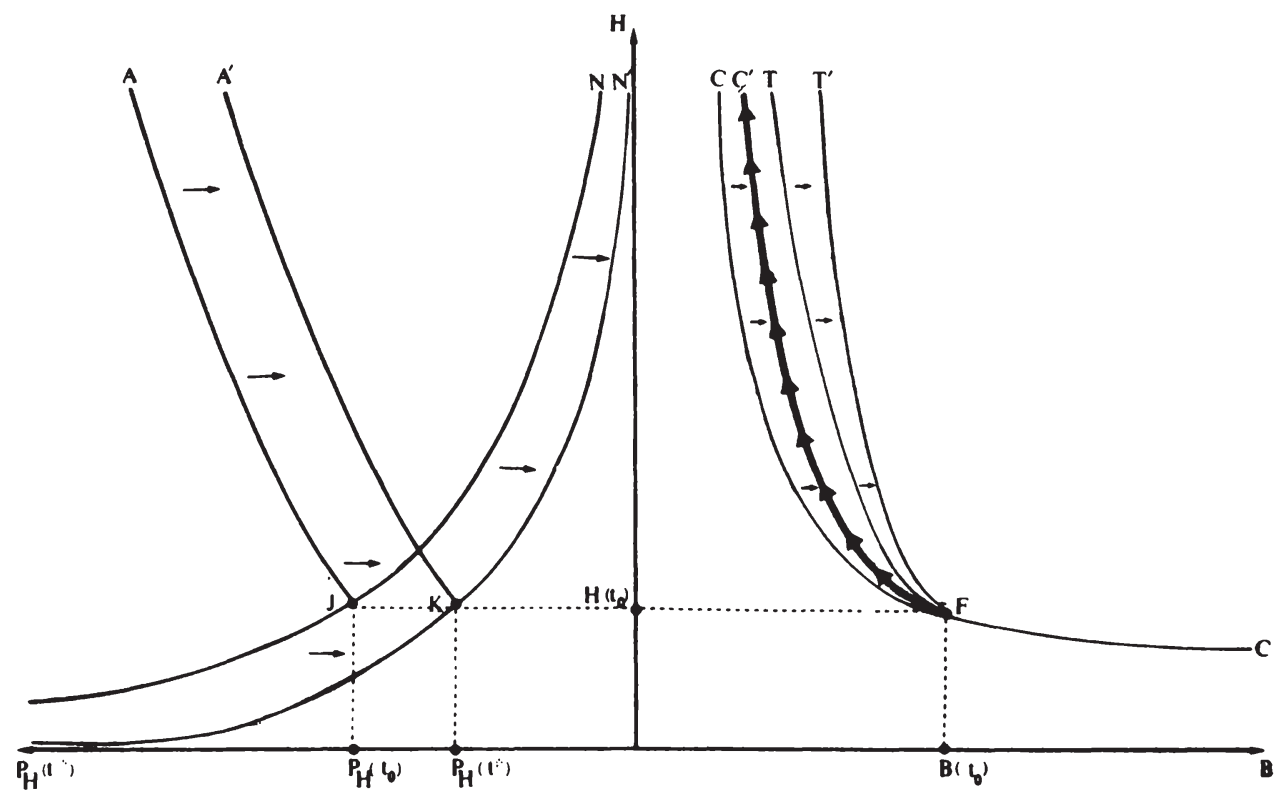

(III.134)

$$
H(t *)=\Phi\left\{\varphi\left[\varphi^{\prime-1}\left(\frac{1-\tau_{e} e_{2}^{\left(\gamma_{1} \rho_{1}+\left(1-\gamma_{2}\right) \rho_{2}-\lambda\right.}}{P_{H}\left(t^{*}\right)}\right)\right]\right\} .
$$

Unter Berücksichtigung der Ableitungseigenschaften $\Phi^{\prime}, \varphi^{\prime},-\varphi^{\prime}$ < 0 folgt aus (III.134), daß der gleichgewichtige Wohnflächenbestand bei beliebig vcrgegebenem Niveau des Häuserpreises $P_{H}$ steuerbedingt größer ist als in der LaisserFaire-Welt.

In Abb. 15 sind diese steuerinduzierten Verschiebungen von Angebots- und Nachfragekurve eingezeichnet. Uberdies sind in Abb. 15 auch die langfristigen Allokationseffekte berücksichtigt, für die die Einkommensbesteuerung des Bodenmaklers verantwortlich zeichnet: der isoelastische Entwicklungspfad FC' und die Sprungkurve FT' weisen in Punkt F - dem zeitpunkt der Einfüh- 
rung der Steuer - einen Knick auf.

Wie der vermietende Wohnungsinvestor kunzfristig auf die Besteuerung seiner Einkünfte aus Vermietung und Verpachtung reagiert, läßt sich nicht eindeutig feststellen. Eine korrekte Antwort auf diese Frage läßt sich nur dann geben, wenn man weiB, welcher der beiden gegenläufigen Effekte - der aus der Subventionierung des Grundstückserwerbs und der steuerlichen Absetzbarkeit abnutzungsbedingter Substanzverluste resultierende, die Vermögensanlage "Wohnimmobilie" begünstigende oder der aus der Nichtabsetzbarkeit abnutzungsbedingter wertverluste des Grundstücksbestandes folgende, die Vermögensanlage in Wohnimmobilien diskriminierende - letztendlich dominiert.

In Abb. 15 ist der Sonderfall festgehalten, daß sich beide Effekte gerade ausgleichen. Mit kurzfristigen Reaktionen ist auf dem Markt für Wohnungsneubau deshalb auch nicht zu rechnen das System wird sich auch nach Einführung der steuer entlang des Pfades FC' weiterentwickeln.

Denkbar sind aber auch Szenarien, in denen die Rechtsverschiebung der Nachfragekurve stärker bzw. schwächer ausfällt als die Rechtsverschiebung der Angebotskurve:

- im ersten Fall wïrde der Wohnungsinvestor seine Neubautätigkeit vorübergehend einstellen und sie erst $\mathrm{zu}$ dem zeitpunkt wieder aufnehmen, in dem die durch die Einkommensbesteuerung entstandene Rentabilitätseinbuße der Wohnungsinvestition durch den Rentabilitätszuwachses ausgeglichen wird, für den das nachfragebedingt permanent ansteigerde Mjetenniveau verantwortlich ist;

- im zweiten Fall uberwiegt dagegen der positive EinfluB von Erwerbssubventionen und Abschreibungsvorteilen. Entsprechend wird der vermietende Wohnungsinvestor bestrebt sein, den in $t_{0}$ vorhandenen Wohnflächenbestand möglichst schnell an den neuen, unter dem EinfluB der Besteuerung gestiegenen gleichgewichtigen Wohnflächenbestand $H\left(t^{*}\right)$ anzupassen: wir werden auf dem Markt für Wohnungsneubau einen kurzfristigen Boom beobachten können, nach dessen Abebben zwar das Niveau der Marktmieten gesunken, das Niveau der Baulandpreise aber gestiegen sein wird. 
Da die Besteuerung der. Einkünfte aus Vermietung und Verpachtung keinen EinfluB hat auf die zeitlichen Veränderungsraten unserer Modellökonomie - dies folgt aus den Bedingungen (III.131b) und (III.132) -, wird durch diese kurzfristigen Reaktionen zugleich auch die langfristige Inzidenz der aktuellen Einkommensbesteuerung vermietender Wohnungsinvestoren bestimmt:

- besteht die Reaktion in kurzer Frist in einem vorübergehenden Neubaustopp, so wird der Wohnflächenbestand $\mathrm{H}$ zu jedem Zeitpunkt jenseits von $t^{*}$ geringer, der Bestand baureifer Grundstücke dagegen zu jedem dieser zeitpunkte größer sein als es bei einem Verzicht auf die Besteuerung der Mieteinkünfte des Wohnungsinvestors der Fall gewesen wäre. Der Zeitpfad des Mietzinses und auch der des Häuserpreises werden infolge des Neubaustopps dauerhaft angehoben;

- reagiert der Wohnungsinvestor auf die Erhebung der Einkommensteuer stattdessen mit einer Intensivierung seiner Neubautätigkeit, so wird das Angebot an Wohnungsnutzungen nach Einführung der Steuer dauerhaft zunehmen, das Niveau der Marktmieten und das des Häuserpreises bei gegebener Entwicklung der Nachfrage nach Wohnleistungen dementsprechend dauerhaft sinken. Sinken werden auch die Zeitpfade des Bestandes baureifer Grundstücke und des Angebots an Bauland. Dies hat wiederum zur Folge, daß der Zeitpfad des Bodenpreises steuerbedingt angehoben wird.

Uncbhärgig von der kurzfristigen Reaktion des Wohnungsmarktes wird es sich für den wohnungsinvestor aufgrund der steuerlichen Diskriminierung des Einsatzes von Bauland bei der Produktion von wohnflächeneinheiten immer lohnen, nach Einführung der steuer eine höhere Kapitalintensität der Bebauung zu wählen. 
5.2.2.2.2. Der Wohnungsinvestor als Selbstnutzer

Während es sich bei der Uberschußrechnung nach $\$ 21$ EStG grosso modo um die auch in anderen sektoren übliche Form der Einkunftsermittlung handelt, stellt die Ermittlung des Nutzungswertes der Wohnung mittels pauschalierter Durchschnittssätze ein einkommensteuerliches Spezifikum des Wohnungssektors dar.

Die Ermittlung des Nutzungswertes auf der Basis des Mietwertes $\mathrm{mH}$ ist nicht mehr möglich, wenn der Wohnungsinvestor sein Wohnungseigentum selbst nutzt, anstatt es (zumindest teilweise) $z$ vermieten. In diesem Falle schreibt das Gesetz ein Verfahren vor, mit dessen Hilfe man den Nutzungswert auf der Basis des Einheitswertes schätzungsweise ermitteln will ( $\$ 21 \mathrm{a}$ EStG); der pauschalierte Nutzungswert für das selbstgenutzte Wohnungseigentum beträgt nach Maßgabe dieses Verfahrens auf das Jahr gerechnet 1 Prozent des (durch den vierzigprozentigen Wertzuschlag) korrigierten, auf den Wertverhältnissen vom 1.1.1964 beruhenden Einheitswertes der Wohnung (\$121a BewG).

Dieser pauschalierte Nutzungswert ist, folgt man der ursprünglichen Intention des Gesetzgebers, in vollem Umfang dem zu versteuernden Einkommen des Wohnungsinvestors zuzuführen. Steuermindernde Abzüge können grundsätzlich nur in Höhe dieses pauschalierten Grundbetrages geltend gemacht werden.

Die pauschale Schätzung des aus der Selbsnutzung von Wohnungseigentum fließenden imputed income ist auch in der finanzwissenschaftlichen Literatur zum Teil heftiger Kritik ausgesetzt. Bemerkenswert hierbei ist, daß die Urteile, in denen die Kritik gipfelt, durchaus konträr sind. So sehen manche in der Nichtabzugsfähigkeit von Schuldzinsen eine nicht zu rechtfertigende steuerliche Diskriminierung des selbstnutzenden Wohnungsinvestors $^{85}$ ), für andere wiederum bedeutet der \$21a EStG eine eindeutige Begünstigung der Selbstnutzer von Wohnungseigentum. 86 )

85) Vgl. z.B. FthLENKEMPER (1982),

86) Vgl. z.B. MUSGRAVE/MUSGRAVE/KULJMER ( 1978 ) . 
Die Argumente, die diese Urteile untermauern sollen, sind jedoch in aller Regel unscharf und verkennen sehr häufig auch den ökonomischen Charakter des Pauschalierungsverfahrens. Diese Mängel sind umso erstaunlicher, als der Gesetzgeber schon bei der Einfuhrung dieser Regelung mitteilte, welche Vorstellungen er mit der Schätzung des Nutzungswertes verbindet.

Die Zulassung von Durchschnittssätzen soll vor allem der verwaltungstechnischen Vereinfachung der Ermittlung fiktiver Mieteinnahmen dienen 87) Dabei "muß für den Nutzungswert der Wohnung im eigenen Haus ein Betrag angesetzt werden, bei dem unter Berücksichtigung des regelmäBigen Aufwandes für das Haus, auf längere zeit gesehen, für den Inhaber immer noch eine angemessene Verzinsung des im Haus angelegten Kapitals bleibt. Als angelegtes Kapital ist nach der Verordnung der Einheitswert angenommen worden." 88 )

Welche Uberlegungen sich hinter der Wendung "angemessene Verzinsung des im Haus angelegten Kapitals" verbergen, geht aus der Gesetzesbegründung zwar nicht explizit hervor; offenbar aber sollte dem Wohnungsinvestor jene reale verzinsung seines in Wohnimmobilien gebundenen Vermögens unterstellt werden, in deren GenuB er durch eine alternative Anlage dieser Aktiva am Kapitalmarkt hätte kommen können.

Im Rahmen unserer Analyse entspricht dem in das Grundstück investierte Eigenkapital der Ertragswert des Grundstücks abzüglich des bei der Finanzierung eingesetzten Fremdkapitals. Mit $r$ als aktuellem Kapitalmarktzins müßten die pauschal geschätzten Einkünfte aus Vermietung und Verpachtung NW vor steuer demnach den D-Mark-Betrag

$$
N W=r\left(P_{H} H-D\right)
$$

87) Vgl. ROGGE (1937), REICHSFINANZMINISTERTUM(1937).

88) Vgl. ROGGE (1937), S. 613. 
annehmen, wobei $\mathrm{P}_{\mathrm{H}} \mathrm{H}$ für den aktuellen Marktwert des Wohnimmobilienvermögens - geschätzt durch ein zeitnahes Ertragswertverfahren - und D für die in diesem Ertragswert enthaltene Fremdkapitalsumme stehen. Der pauschalierte Nutzungswert aus (III.135) unterliegt der Einkommensteuerpflicht. Entsprechend muß nun auch der Wohnungsinvestor den an den Fiskus abzuführenden Geldbetrag $\tau_{e} N W$ in seinen Planungskalkül miteinbeziehen. Er steht auch hier wieder vor dem Problem, durch eine optimale wahl der Zeitpfade der Nettoneuverschuldung, des Erhaltungsaufwandes, des Bodenverbrauchs und der Kapitalintensität der Bebauung den Marktwert seines Wohnungsunternehmens $\mathrm{zu}$ maximieren:

(III. 136)

$$
\left\{S, E, \varepsilon, F^{d}\right\} M_{W}^{M}=\int_{t_{0}}^{\infty} A(t) \exp \left[-z \theta_{e} r\left(t-t^{*}\right)\right] d t
$$

unter den Bedingungen (II.2), (II.3), (II.9)', (II.10), (II.14), (II.16), (II.17a) und

(III.137)

$$
\begin{aligned}
A= & z\left[m H-E-r D-\tau_{e} r\left(P_{H} H-D\right)\right]+S \\
& -\left[1-\tau_{e}\left(\gamma_{2} \rho_{1}+\left(1-\gamma_{2}\right) \rho_{2}\right)\right]\left(F^{d} \varepsilon-P_{B} F^{d}\right) .
\end{aligned}
$$

In (III.137) ist zusätzlich die Tatsache berücksichtigt, daß auch der selbstnutzende Wohnungsinvestor Wohnungseigentum auf drei unter Kostengesichtspunkten unterschiedlichen Wegen erwerben kann.

Die in laufenden Werten formulierte Hamiltonfunktion für dieses Problem lautet

(III. 138)

$$
\begin{aligned}
H= & z\left(m H-E-\Theta_{e} r D-\tau_{e} r P_{H}^{H}\right)+S \\
& -\left[1-\tau_{e}\left(\gamma_{2} \rho_{1}+\left(1-\gamma_{2}\right) \rho_{2}\right)\right]\left(F^{d} \varepsilon-P_{B} F^{d}\right) \\
& +P_{H}\left[F^{d} \varphi(\varepsilon)-z(\alpha \delta H-E)\right] \\
& +\lambda_{D} S .
\end{aligned}
$$


Wie man durch Differentiation von (III.138) nach der Kontrollvariablen S leicht überprüfen kann, ist der Wohnungsinvestor auch im Falle der (Idealform der) Ermittlung des Nutzungswertes mit Hilfe pauschalierter Durchschnittssätze indifferent bezüglich der Entscheidung, ob er seine wohnungsinvestition mit Fremdkapital oder mit Eigenkapital finanzieren soll; da auch bei der Besteuerung des Nutzungswertes aus (III.139) Fremdkapitalzinsen in voller Höhe steuermindernd geltend gemacht werden können und die Erträge anderer Investitonsmöglichkeiten ebenfalls der Einkommensbesteuerung unterliegen, böte eine (marginale) Änderung in der Finanzierungsstruktur zugunsten der einen oder der anderen Finanzierungsform keinen Rentabilitätsvorteil.

Ebensowenig ändern wird sich infolge der pauschalen Schätzung des Nutzungswertes der eigenen wohnung der paretooptimale werterhaltungsplan des selbstnutzenden Wohnungsinvestors. Wie aus (III.135) hervorgeht, wird der Erhaltungsaufwand bei der Schätzung des Nutzungswertes nicht berücksichtigt; der Nettopreis für eine Einheit Erhaltungsaufwand wird deshalb vor und nach Steuer 1 D-Mark betragen. Da der Preis $P_{H}$ einer Wohnflächeneinheit annahmegemäß zu jedem Zeitpunkt des Planungshorizonts über 1 D-Mark liegt, bedeutet dies, daß der abnutzungsbedingte Substanzverlust des Gebäudekapitalstocks in jeder Periode in vollem umfang durch werterhaltende Arbeiten ausgeglichen wird.

Die restlichen Bedingungen für das Gleichgewicht nach Steuer erhält man durch partielle Differentiation der Hamiltonfunktion aus (III.138) nach den Kontrollvariablen $\varepsilon$ und $F^{d}$ sowie nach der Zustandsvariablen $H$. Es gilt

$$
P_{H^{\prime}} \varphi^{\prime}(\varepsilon)=1-\tau_{e}\left(\gamma_{2} \rho_{1}+\left(1-\gamma_{2}\right) \rho_{2}\right) \text {, }
$$

(III. 140)

$$
\frac{B}{\alpha} \varepsilon\left\{\begin{array}{l}
<\} \\
=j
\end{array} P_{B} \Rightarrow F^{D}\left\{\begin{array}{l}
= \\
\geq
\end{array}\right\} 0\right.
$$

(III. $141 \mathrm{a}$ )

$$
\hat{\mathrm{P}}_{\mathrm{H}}=\mathrm{O}
$$

für $t_{0} \leq t<t^{*}$,

(III. 141b)

$$
\hat{\mathrm{P}}_{\mathrm{H}}=-\frac{\mathrm{m}}{\mathrm{P}_{\mathrm{H}}}+r+a \delta \text { für } t \geq t^{*} \text {. }
$$


Die Bedingungen (III.139) bis (III.141b) sind der mathematische Beleg dafür, daß mit der Einkommensbesteuerung des Selbstnutzers eventuell elnhergehende Allokationsverzerrungen alleine auf die die Erwerbsphase betreffenden Mängel des geltenden Einkommensteuerrechts zurückgehen, wenn der Nutzungswert (idealtypisch) in der in (III.135) beschriebenen Art und Weise ermittelt wird; je nachdem, ob der selbstnutzer sein Wohnungseigentum von einem nicht zur versteuerung aufgedeckter stiller Reserven verpflichteten Bauherrn oder selbst in der Funktion des Bauherrn erworben hat, subventioniert die öffentliche Hand die Ausgaben für Bauleistungen und Bauland in unterschiedlich starkem Maße; Bedingung (III.139) bringt zum Ausdruck, daß aus diesem Grunde die im Gleichgewicht an das Wertgrenzprodukt des Produktionsfaktors "Bauleistungen" gestellten Anforderungen einkommensteuerbedingt abnehmen. Da die Kapitalintensität der Bebauung sich unter dem Einfluß dieser Subventionierung nicht ändert - wie aus (III. 140) hervorgeht, profitieren beide Produktionsfaktoren von den öffentlichen Zuschüssen in gleichem Maße - läßt sich das steuerbedingt sinkende Wertgrenzprodukt des Faktors "Bauleistungen" nur durch sinkende Häuserpreise respektive durch einen steuerbedingt zunehmenden Wohnflächenbestand erklären.

Uberraschender als dieses Ergebnis - steigende Investitionsbereitschaft im Wohnungssektor aufgrund einer de-facto-Beteiligung der öffentlichen Hand an den Anschaffungs- bzw. Herstellungskosten selbstgenutzter Wohnungen - ist sicherlich die Tatsache, daß wir mit der Besteuerung des in (III. 135) beschriebenen pauschalierten Nutzungswertes eine weitere Möglichkeit gefunden haben, Einkünfte aus der Vermietung und Verpachtung von Wohnungseigentum $\mathrm{zu}$ besteuern, ohne dadurch die Bedingungen für eine effiziente Allokation der Faktoren "Bauleistungen" und "Bauland" zu verletzen. Dieses Ergebnis erlaubt uns die Formulierung der folgenden Hypothese: wenn die Finanzbehörden über ein zeitnahes Verfahren zur Bewertung von Grundstücken verfügen, den Abzug von Fremdkapitalzinsen ohne Einschränkung gestatten und bei der Pauschalierung 
einen Multiplikator verwenden, der mit dem Kapitalmarktzins übereinstimmt, ist die Besteuerung des pauschalierten Nutzungswertes der Wohnung allokationsneutral.

Die Neutralität der Pauschalbesteuerung folgt unmittelbar aus der Tatsache, daß die steuerbemessungsgrundlage aus (III.135) den ökonomischen Reinvermögenszugang des Wohnungsinvestors korrekt widerspiegelt. Der Wohnungsinvestor bzw. der Haushalt, dessen Interessen er wahrnimmt, steht zu Beginn einer jeden Periode vor der Entscheidung, entweder seinen gesamten Wohnflächenbestand zum Preis $\mathrm{P}_{\mathrm{H}} \mathrm{H} z \mathrm{z}$ verkaufen, seine Schulden zu tilgen und den Restbetrag am Kapitalmarkt anzulegen, um in der nächsten Periode $\mathrm{Z}$ inseinkünfte in Höhe von $r\left(\mathrm{P}_{\mathrm{H}} \mathrm{H}-\mathrm{D}\right) \mathrm{D}$-Mark realisieren zu können oder aber seinen Wohnungsbestand $\mathrm{zu}$ halten und sich mit dem auf diese Weise erzielbaren Reinvermögenszugang in Höhe von $\left[\mathrm{mH}+\left(\mathrm{P}_{\mathrm{H}}^{\cdot} \mathrm{H}\right)-\mathrm{rD} \mathfrak{i} \mathrm{zu}\right.$ begnügen. Das durch Bedingung (III 141b) beschriebene Portefeuillegleichgewicht setzt voraus, daB der Wohnungsinvestor zu jedem Zeitpunkt zwischen beiden Alternativen indifferent ist. Okonomisch rational ist dieses Verhalten aber nur dann, wenn die Renditen beider Alternativen dieselben sind. Entsprechend wird es für den Wohnungsinvestor auch keinen Unterschied machen, ob er in einer Periode nun den pauschal geschätzten steuerbetrag $\tau_{e} e\left(P_{H} H-r D\right)$ oder den im Rahmen einer korrekten, der Konzeption des Reinvermögenzuganges folgenden Uberschußrechnung ermittelten Betrag $\tau_{e}\left(m H+\left(P_{H} H\right)-r D\right)$ entrichten muß. In beiden Fällen wird er an der Last der Einkommensteuer gleich schwer tragen.

Unsere Analyse verhilft uns darüberhinaus $\mathrm{zu}$ einer weiteren, für unsere folgenden Uberlegungen aufschlußreichen Erkenntnis: der pauschalierte Nutzungswert der selbstgenutzten Wohnung darf zu keinem zeitpunkt negativ werden. Und nur für den Fall, daß das gesamte Neubauvolumen zu 100 Prozent mit Fremdkapital finanziert wird und auch die sich im Laufe der zeit einstellenden Wertzuwächse des Grundstückvermögens in voller Höhe beliehen werden, darf der korrekt pauschalierte Nutzungswert gerade 
gleich Null sein, wenn diese Variante der Einkunftsermittlung allokationsneutral sein soll. Sieht man von diesem Grenzfall einmal ab, so gelangt man zu dem Ergebnis, daß eine effiziente Besteuerung fiktiver Mieteinnahmen notwendigerweise $z u$ jedem zeitpunkt streng positive pauschalierte Nutzungswerte voraussetzt.

Vor allem diese letzte Bedingung ist Ursache für viele Mißverständnisse in der Beurteilung des ökonomischen Charakters des Pauschalierungsverfahrens. So wird im wohnungspolitischen Tagesgeschäft regelmäßig die Meinung vertreten, daß cas Steuergesetz den Vermieter gegenüber dem selbsnutzer bevorzuge, da es letzterem nicht möglich sei, über den Ansatz von Fremdkapitalzinsen und steuerlich zulässiger AfA negative Einkünfte aus Vermietung und Verpachtung $z u$ erzielen und auf diesem Wege in den Genuß einer Einkommensteuerrückerstattung $z u$ gelangen.

Daß dieses Urteil im Lichte einer unter Rentabilitätsgesichtspunkten vorgenommenen Analyse nicht aufrechterhalten werden kann, läßt sich durch unsere vorangegangenen Untersuchungen belegen. Während das idealtypische Pauschalierungsverfahren die Rentabilität der Wohnungsinvestition nicht berührt, läßt sich hinsichtlich des praktizierten Uberschußverfahrens nicht mit letzter sicherheit sagen, ob es eine Vermögensanlage in Form von Wohnimmobilien gegenüber anderen Anlageformen begünstigt oder benachteiligt.

Alleine der mit der UberschuBrechnung einhergehende Liquiditätsvorteil - tatsächlich sind die gemä $B$ den Bestimmungen des §21 EStG ermittelten Einkünfte aus Vermietung und Verpachtung in cier Anfangsphase der Nutzung meistens negativ - kann zur Unterstützung der These herangezogen wercen, daß das geltende Einkommensteuerrecht die Schaffung nicht (vollständig) eigengenutzten Wohnraums bevorzuge.

Welch nachteilige Folgen für die gesellschaftliche Wohlfahrt eine solch einseitige Reduzierung der Förderproblematik auf den Liquiditätsaspekt nach sich ziehen kann, zeigen 
die Reaktionen des Gesetzgebers. So weicht das derzeit praktizierte Verfahren der Nutzungswertpauschalierung in allen wesentlichen Punkten von dem oben beschriebenen Idealtypus ab.

An erster Stelle ist hier die systematische Fehlschätzung des Nutzungswertes der Wohnung zu nennen. Dieser Mangel hat seine Ursache vor allem in den uns aus der Grundsteueranalyse bekannten Unzulänglichkeiten der angewandten Bewertungsverfahren; sie führen dazu, daß der Einheitswert des Grundstücks meist erheblich unter dem betreffenden Verkehrswert liegt ${ }^{90}$ ) Zudem wurde mit dem einprozentigen Pauschalierungssatz wohl auch ein zu kleiner Multiplikator gewählt.

Auf die Qualität der Schätzung wirkt sich darüberhinaus die Tatsache aus, daß bei der Pauschalierung keine Rücksicht genommen wird auf die Finanzierungsstruktur der Wohnimmobilie: der auf der Basis der Jahresrohmiete ermittelte Einheitswert soll den Marktwert des gesamten in dem Grundstück gebundenen Vermögens widerspiegeln. Entsprechend handelt es sich bei dem $\mathrm{zu}$ versteuernden Nutzungswert um die (unterstellte) Rendite dieses Vermögens. Den Ansatz effektiv gezahlter Schuldzinsen sieht §21a EStG grundsätzlich nicht vor.

90) Bemerkenswert ist hierbei auch, daß trotz gleicher Baukosten der Einheitswert eines Zweifamilienhauses im allgemeinen geringer ist als der eines Einfamilienhauses; dies deshalb, weil der laut Bewertungsrecht auf das Einfamilienhaus anzuwendende Multiplikator und oft auch die für das Einfamilienhaus unterstellte Rohmiete größer sind als beim Zweifamilienhaus. Dabei brauchen die beiden Gebäude in ihrer baulichen Gestaltung noch nicht einmal sehr voneinander abzuweichen; denn die Konstruktionsmerkmale, die nach Vorgabe des Bewertungsgesetzes ein Einfamilienhaus von einem Zweifamilienhaus unterscheiden, sind vergleichsweise bedeutungslos. Diese bewertungsrechtliche Differenzierung wird noch unverständlicher, wenn man bedenkt, daß die beiden kinheitswerte ' in aller Regel übereinstimmen, wenn sie nach dem Sachwertverfahren ermittelt worden sind. Wie immer man diese Diskriminierung aus politischer Sicht rechtfertigen mag - unter allokationstheoretischen Gesichtspunkten machen sie wenig Sinn: die Eigennutzung wird steuerlich unterschiedlich behandelt, je nachdem, ob der Wohnungsinvestor beschlossen hat, in einem Einfamilienhaus oder in einem Zweifamilienhaus $\mathrm{zu}$ wohnen. 
Führt man sich die Konsequenzen dieser Mängel vor Augen, so läßt sich a priori nicht sagen, ob der pauschalierte Nutzungswert der selbstgenutzten Wohnung durch das praktizierte Schätzverfahren $\mathrm{zu}$ hoch oder $\mathrm{zu}$ niedrig ausgewiesen wird: während das geltende Bewertungsrecht als Ursache für eine mögliche Unterschätzung angesehen werden muß, wird die Nichtberücksichtigung von Fremdkapitalzinsen tendenziell zu einer Uberschätzung des Nutzungswertes führen.

Probleme, die sich aufgrund dieser Schätzungsgenauigkeiten ergeben könnten, werden durch eine andere Bestimmung des Einkommensteuergesetzes vermieden. So hat der Gesetzgeber in $\$ 21 \mathrm{a}$, Absatz 3, Ziffer 1 EStG einen (zusätzlichen) Werbungskostenabzug in Höhe des pauschalierten Grundbetrags erlaubt; ${ }^{91}$ ) diese Möglichkeit führt erfahrungsgemäB dazu, daB der selbstnutzende Wohnungsinvestor per saldo keine $z u$ versteuernden Einkünfte aus Vermietung und Verpachtung erzielt. Erinnern wir uns an die aus der Analyse des idealtypischen Pauschalierungsverfahrens gewonnene Erkenntnis, daB ein $z u$ versteuernder Nutzungswert in Höhe von Null nur dann mit den Erfordernissen einer effizienten Einkommensbesteuerung in Einklang $z u$ bringen ist, wenn in der Wohnimmobilie keine einzige D-Mark Eigenkapital gebunden ist, so kommen wir zu dem Ergebnis, daB die Bestimmung des $\$ 21 a$, Absatz 3, ziffer 1 EStG - für sich betrachtet und verglichen mit der steuerfreien Welt - als eine steuerliche Begünstigung des "Wohnens im eigenen Heim" $z$ u werten ist. Sie fällt umso stärker aus, je weniger Fremdkapital der Wohnugnsinvestor zur Finanzierung seiner Wohnungsinvestitionen aufnimmt.

91) Entgegen den Bestimmungen des $\$ 21 \mathrm{a}$, Absatz 3, Ziffer 1 EStG stellen nicht nur Fremdkapitalzinsen abzugsfähige Werbungskosten dar. Wie die Praxis zeigt, werden als Werbungskosten fast ausschließlich Aufwendungen geltend gemacht, die im zuge der erstmaligen Durchführung einer Maßnahme im Sinne des §82a, Absatz 1 EStDV angefallen sind (beispielweise Aufwendungen für die erstmalige Isolierverglasung oder Wärmedämmung) . 
Wohl unter dem Einciruck der Tatsache, daß alich ein auf die eben beschriebene Art modifiziertes Pauschalierungsverfahren nicht zu negativen Einkünften aus Vermietung und Verpachtung bzw. zu dem damit verbundenen Liquiditätsvorteil verhelfen kann, hat der Gesetzgeber zwei zusätzliche Möglichkeiten geschaffen, die nun auch im Rahmen der Nutzungswertpauschalierung nach §21a EStG zu steuerwirksamen Verlusten führen können:

- so ist es dem selbstnutzenden Wohnungsinvestor seit Gültigkeit des Zweiten Haushaltstrukturgesetzes möglich, über den Grundbetrag hinaus jährlich Fremdkapitalzinsen in Höhe von maximal 10000 D-Mark steuermindernd geltend zu machen. Da diese auf drei Jahre befristete Regelung wohl eher konjunkturpolitisch, also nicht fiskalisch, motiviert ist, soll sie in unserer Analyse nicht berücksichtigt werden;

- der zweite Weg, auf dem der selbstnutzende Wohnungsinvestor zu negativen Einkünften aus Vermietung und Verpachtung gelangen kann, wird durch §21a, Absatz 3, Ziffer 2 EStG gewiesen: dieser Bestimmung zufolge kann auch der selbstnutzer erhöhte Absetzungen nach $\$ 7 \mathrm{~b}$ EStG in Anspruch nehmen, nach Abzug der Werbungskosten und über den Grundbetrag hinaus. Im Unterschied zum Vermieter ist es dem Selbstnutzer lediglich gestattet, die erhöhten Absetzungen während der Begünstigungsphase - also 5 Prozent pro anno während der ersten 8 Nutzungsjahre - geltend zu machen. Er kann demnach 40 prozent der (begrenzten) Herstellung- bzw. Anschaffungskosten des Gebäudes steuerlich absetzen.

రkonomisch betrachtet beschreibt §21a, Absatz 3, Ziffer 2 EStG einen verlorenen öffentlichen ZuschuB auf die Anschaffungsbzw. Herstellungskosten des Gebäudes zum Zeitpunkt der Anschaffung bzw. Fertigstellung. Als solchen wollen wir die erhöhten Absetzungen des $\$ 21 \mathrm{a}$ EStG auch in unsere Analyse aufnehmen: wir unterstellen, daß der öffentliche sektor an den Gestehungskosten $F_{\varepsilon}^{d}$ des selbstgenutzten Gebäudes in Höhe des Betrages $\tau_{e} \rho_{3} F^{d} \varepsilon$ partizipiert (wobei infolge der Barwertdefinition dieser Vergünstigung $\rho_{3}<0,4 \mathrm{gilt}$ ). 
Aufbauend auf den vorangegangenen Ausführungen wissen wir, da $\beta$ das imputed income des selbstnutzenden Wohnungsinvestors de facto nicht der Einkommensbesteuerung unterliegt. Folgerichtig können dann aber auch Fremdkapitalzinsen nicht steuermindernd geltend gemacht werden. Unter Berücksichtigung des Vermögenstransfers $\tau_{e} \rho_{3} F_{\varepsilon}^{d}$ und in Kenntnis der Tatsache, daß auch dem selbstnutzenden Wohnungsinvestor drei Wege des Wohnungseigentumerwerbs offenstehen, kann deshalb in jeder Periode ein Betrag in Höhe von

(III.142)

$$
\begin{aligned}
A= & z(m H-E-r D)+S \\
& -\left[1-\tau_{e}\left(\gamma_{2} \rho_{1}+\left(1-\gamma_{2}\right) \rho_{2}\right)\right]\left(F_{\varepsilon}^{d}-P_{B} F^{d}\right) \\
& +\tau_{e} \rho_{3} F^{d}
\end{aligned}
$$

D-Mark entnommen werden.

In Analogie zur bisherigen Vorgehensweise stellt sich als nächstes die Aufgabe, durch eine entsprechende Planung der Finanzierung, der Werterhaltung und des Wohnungsneubaus den Barwert des Entnahmestromes - den Marktwert des Wohnungsunternehmens - zu maximieren; auch hierbei müssen die Restriktionen (II.2), (II.3), (II.9), (II.10), (II.14), (II.16), und (II.17a) berücksichtigt werden. Man gelangt auf diesem Wege zu der Hamiltonfunktion

(III.143)

$$
\begin{aligned}
H= & z(m H-E-r D)+s+\tau_{e} \rho_{3} F^{d} \varepsilon \\
& -\left[1-\tau_{e}\left(\gamma_{2} \rho_{1}+\left(1-\gamma_{2}\right) \rho_{2}\right)\right]\left(F_{\varepsilon}^{d}-P_{B} F^{d}\right) \\
& +P_{H}\left[F d^{d}(\varepsilon)-z(\alpha \delta H-E)\right] \\
& +\lambda_{D} S .
\end{aligned}
$$


Zur Bestimmung der optimalen Finanzierungsform muB (III.143) partiell nach der Nettoneuverschuldungsvariablen $\mathrm{S}$ differenziert werden. Man errechnet leicht

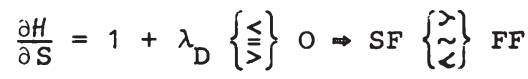

Um $z u$ einem eindeutigen Urteil über die Finanzierungspräferenzen des Wohnungsinvestors $\mathrm{zu}$ gelangen, mu $\mathrm{B}$ man den Wert des Schattenpreises $\lambda_{D}$ kennen; für ihn gilt

$$
\lambda_{D}=\frac{\partial M_{W}}{\partial D}=-\frac{1}{\Theta_{e}}<-1 .
$$

Setzt man (III.145) in (III.144) ein, so kommt man zu dem Ergebnis, daß das gegenwärtig praktizierte system der Nutzungswertpauschalierung beim wohnungsinvestor eine eindeutige präferenz für die selbstfinanzierung entstehen läßt.

Diese Abneigung gegen eine Finanzierung mit Fremdkapital läßt sich anhand einer einfachen Arbitrageüberlegung begründen. Würde der Wohnungsinvestor nämlich weiterhin mit Krediten finanzieren, könnte er wegen der de facto fehlenden Besteuerung der Mieteinnahmen auch Fremdkapitalzinsen nicht mehr steuermindernd geltend machen. Für jede aufgenommene D-Mark müBte er deshalb $\mathrm{z}$ insen in Höhe von $r$ D-Mark zahlen. Durch die Entscheidung für die Selbstfinanzierung gelingt es ihm stattdessen, die reinen Finanzierungskosten seiner Wohnlingsinvestitionen auf demselben Niveau zu halten, das diese im Rahmen der Uberschußrechnung bei Abzugsfähigkeit der Fremdkapitalzinsen innehaben: für jede in Wohnungseigentum gebundene D-Mark Eigenkapital erwachsen ihm Opportunitätskosten in Gestalt entgangener Kapitalmarkterträge in Höhe von $\Theta_{e}$ D-Mark.

Obwohl die Fremdfinanzierung als optimale Finanzierungsform ausscheidet, wenn Einkünfte aus Vermietung und Verpachtung pauschal geschätzt werden, muß ein "armer" Wohnungsinvestor, der nicht über ausreichendes Eigenkapital verfügen kann, diesen vergleichsweise teuren Finanzierungsweg beschreiten. Es steht 
zu erwarten, daß dieses Auseinanderfallen von Finanzierungspräferenzen und Finanzierungsmöglichkeiten auch Auswirkungen hat auf seine Investitionsentscheidungen.

Um den Einfluß aufzuzeigen, den die Finanzplanung auf den Investitionskalkül beider Selbstnutzertypen ausübt, gehen wir aus von den allgemeinen Bedingungen

$$
\begin{array}{ll}
\text { (II.23) } & \frac{\partial H}{\partial E}=\frac{\partial H}{\partial E}+\frac{\partial H}{\partial S} \frac{\partial S}{\partial E}, \\
\text { (II.28) } & \frac{\partial H}{\partial \varepsilon}=\frac{\partial H}{\partial \varepsilon}+\frac{\partial H}{\partial S} \frac{\partial S}{\partial \varepsilon}, \\
\text { (II.29) } & \frac{\partial H}{\partial F} d=\frac{\partial H}{\partial F} d+\frac{\partial H}{\partial S} \frac{\partial S}{\partial F} d, \\
\text { (II.30) } & \frac{\partial H}{\partial H}=-\left[\frac{\partial H}{\partial H}+\frac{\partial H}{\partial S} \frac{\partial S}{\partial H}\right] .
\end{array}
$$

Im Falle des "reichen" Wohnungsinvestors wissen wir, daß dieser in der Lage ist, ảie benötigten Geldbeträge aus eigenen Mitteln aufzubringen. Deshalb wird er weder für den Erwerb baureifer Grundstücke oder den Kauf von Bauleistungen Kredite aufnehmen, noch Wertzuwächses seines Wohnflächenbestandes beleihen. Entsprechend gilt in den oben aufgeführten Bedingungen

$$
\frac{\partial S}{\partial E}=\frac{\partial S}{\partial \varepsilon}=\frac{\partial S}{\partial F} d=\frac{\partial S}{\partial H}=0 .
$$

Berechnet man die infolge von (III.146) vereinfachten partiellen Differentiale, so erhält man die folgenden notwendigen Bedingungen für den gleichgewichtigen Zeitpfad des Erhaltungsaufwandes und des Wohnungsneubauprogramms:

(III. 147)

(III.148)

$$
\begin{aligned}
& E=\left\{\begin{array}{ll}
0 & \text { für } t_{0} \leq t<t^{*} \\
\alpha \delta H & \text { für } t \geq t^{*}
\end{array},\right. \\
& P_{H^{\varphi}} \varphi^{\prime}(\varepsilon)=1-\tau_{e}\left[\gamma_{2} \rho_{1}+\left(1-\gamma_{2}\right) \rho_{2}+\rho_{3}\right],
\end{aligned}
$$


(III. 149)

$$
\frac{\beta}{\alpha} \varepsilon\left[1-\frac{\rho_{3} \tau_{e}}{1-\tau_{e}\left[\gamma_{2} \rho_{1}-\left(1-\gamma_{2}\right) \rho_{2}\right]}\right] \quad\left\{\begin{array}{l}
< \\
=
\end{array}\right\} P_{B} \Rightarrow F^{d}\left\{\begin{array}{l}
= \\
\geq
\end{array}\right\} 0,
$$

(III. 150a)

$$
\hat{\mathrm{P}}_{\mathrm{H}}=0 \quad \text { für } t_{\mathrm{O}} \leq t<t^{*} \text {, }
$$

(III. 150b)

$$
\hat{\mathrm{P}}_{\mathrm{H}}=-\frac{m}{\bar{p}_{\mathrm{H}}}+\Theta_{\mathrm{e}} r+\alpha \delta \text { für } t \geq t *
$$

Wie aus (III.147) hervorgeht, ist das Programm werterhaltender Maßnahmen unter dem Regime des praktizierten Pauschalierungsverfahrens das gleiche wie in der steuerfreien welt - in jeder Periode werden die abnutzungsbedingten substanzverluste des Gebäudekapitals durch Renovierungsarbeiten in vollem umfang kompensiert.

Aus Bedingung (III.148) folgt, daß im neuen Gleichgewicht das Wertgrenzprodukt der letzten im Rahmen der Gebäudeerstellung eingesetzten Bauleistungseinheit gleich sein muß dem für diese marginale Einheit entrichteten Bruttopreis $\left(P_{I}=1\right)$ abzüglich der hierauf entfallenden öffentlichen Subventionen, die sich zusammensetzen aus

- der Erwerbsprämie $\tau_{e}\left[\gamma_{2} \rho_{1}+\left(1-\gamma_{2}\right) \rho_{2}\right]$, die in Abhängigkeit von der Erwerbsform unterschiedlich hoch ausfällt, und

- dem Barwert $\tau_{e} \rho_{3}$ der für die marginale D-Mark zulässigen erhöhten Absetzungen.

Da der Barwert der dem selbstnutzenden Wohnungsinvestor eingeräumten $7 b$-Vergünstigungen zumindest bei dem in dieser Analyse unterstellten Proportionaltarif mit sicherheit geringer ist als der Barwert der Steuerrückerstattungen, die dem vermietenden Wohnungsinvestor infolge der Geltendmachung von Absetzungen für Abnutzung zufließen (es gilt $\tau_{e} \rho_{3}<\lambda_{K^{*}}$ ), fällt die steuerinduzierte Verbilligung der Bauleistungen beim selbstnutzenden Wohnungsinvestor schwächer aus als bei seinem vermietenden Kollegen.

Graphisch drückt sich diese (vergleichsweise geringe) einkommensteuerliche Begünstigung der Aufwendungen für Bauleistungen bei der Errichtung selbstgenutzten Wohnraums dadurch aus, 
daß die Angebotskurve aus Abb. 7 nach rechts rückt, diese Rechtsverschiebung nun aber schwächer ausfällt als im Falle der Uberschußrechnung geltenden Rechts.

Analytisch werden diese Zusammenhänge erkennbar, wenn man die Inverse der Optimalitätsbedingung (III.148) in die allgemeine Form der Angebotsfunktion aus (II.79) substituiert:-man erhält dadurch die Funktion der Angebotskurve $A^{\prime}$ in Abb.16

$$
H\left(t^{*}\right)=\Phi\left\{\varphi\left(\varphi^{\prime}-1\left[\frac{1-\tau_{e}\left(\gamma_{2} \rho_{1}+\left(1-\gamma_{2}\right) \rho_{2}+\rho_{3}\right)}{P_{H}\left(t^{*}\right)}\right]\right)\right\}
$$

- und hierbei die Eigenschaften $\tau_{e} \rho_{3}<\lambda_{K^{*}}$ sowie $\Phi_{,} \varphi^{\prime},-\varphi^{\prime}{ }^{\prime}<0$ beachtet.

Bedingung (III.149) ist zu entnehmen, daß der Einsatz des Produktionsfaktors Boden bei der Errichtung von Wohnraum auch im Falle der pauschalen Schätzung der Einkünfte aus Vermietung und Verpachtung diskriminiert wird. Wie schon bei der Ubberschußrechnung, so ist auch hier als Ursache dieser Diskriminierung der Umstand anzusehen, daß zwar die Anschaffungs- bzw. Herstellungskosten des Gebäudes, nicht aber die Anschaffungskosten des Bauplatzes im Zuge der erhöhten Absetzungen für Abnutzung steuermindernd geltend gemacht werden können. Folge dieser Diskriminierung wird sein, daß unter sonst gleichen Bedingungen die Zahlungsbereitschaft des Wohnungsinvestors für Bauboden sinkt. Falls der Bodenmakler nicht bereit sein sollte, im AnschluB an die Einführung der Pauschalbesteuerung den im Vergleich zum Allokationsoptimum geringeren Bodenpreis

$$
\bar{P}_{B}=P_{B}\left(1-\frac{\tau_{e} \rho_{3}}{1-\tau_{e}\left[\gamma_{2} \rho_{1}+\left(1-\gamma_{2}\right) \rho_{2}\right]}\right)
$$

zu akzeptieren, wird der selbstnutzende Wohnungsinvestor auf die Besteuerung seiner fiktiven Mieteinkünfte mit einer Erhöhung der Kapitalintensität der Bebauung reagieren. 
Sind die allokativen Effekte der Einkommensbesteuerung vermietender und selbstnutzender Wohnungsinvestoren bis hierher zumindest unter qualitativen Aspekten dieselben, so zeigt sich im Einfluß des Pauschalierungsverfahrens auf die Nutzungskosten des gebundenen Vermögens ein entscheidender Unterschied: wie ein Blick auf Bedingung (III.150b) zeigt, sinken die user cost des im Wohnungssektor gebundenen Vermögens im Vergleich zur steuerfreien Welt, wenn Einkünfte aus Vermietung und Verpachtung im Sinne des §21a EStG ermittelt werden; dieses Ergebnis deutet darauf hin, daß das Pauschalierungsverfahren die Attraktivität selbstgenutzten Wohneigentums als spezielle Form der Vermögensanlage im Vergleich $\mathrm{zu}$ allen anderen in unserem Modell betrachteten Vermögensbeständen erhöht. Die faktische Freistellung fiktiver Mieteinnahmen eröffnet dem selbstfinanzierenden und selbstnutzenden Wohnungsinvestor einen legalen steuerfluchtweg - heraus aus der steuerpflichtigen Kapitalmarktanlage und hinein in die steuerfreie Wohnungsinvestition.

Graphisch läßt sich dieser die Wohneigentumsbildung eindeutig begünstigende Effekt durch eine Linksverschiebung der Nachfragekurve aus Abb.7 darstellen; löst man (III.150b) nach $P_{H}$ auf und berücksichtigt dabei, daß der Häuserpreis aufgrund des einkommensteuerbedingt veränderten Angebotsverhaltens des repräsentativen Bodenmaklers mit einer im Vergleich zur LaissezFaire-Welt geringeren Rate wächst [vgl. Bedingung (III.111)], so gilt für die Nachfragekurve $N^{\prime}$ im linken Quadranten von Abb.16 die funktionale Beziehung

$$
P_{H}=\frac{m(a / H)}{\theta_{e}(1-\beta) r+\alpha \delta}
$$

Ein Vergleich mit der entsprechenden Laissez-Faire-Beziehung (dort gilt $\theta_{e}=1$ ) zeigt, daB der Wert des Quotienten in (III.153) wegen $0<\theta_{e}<1$ unter dem EinfluB der steuer zunimmt; $\mathrm{zu}$ jedem beliebigen Mietzins $\mathrm{m}$, infolge der exogen vorgegebenen Nachfrage nach Wohnleistungen also auch $\mathrm{zu}$ jedem gegebenen Wohnflächenbestand $H$, gehört somit ein höherer Häuserpreis $P_{H}$ • 
$\mathrm{Abb} .16$

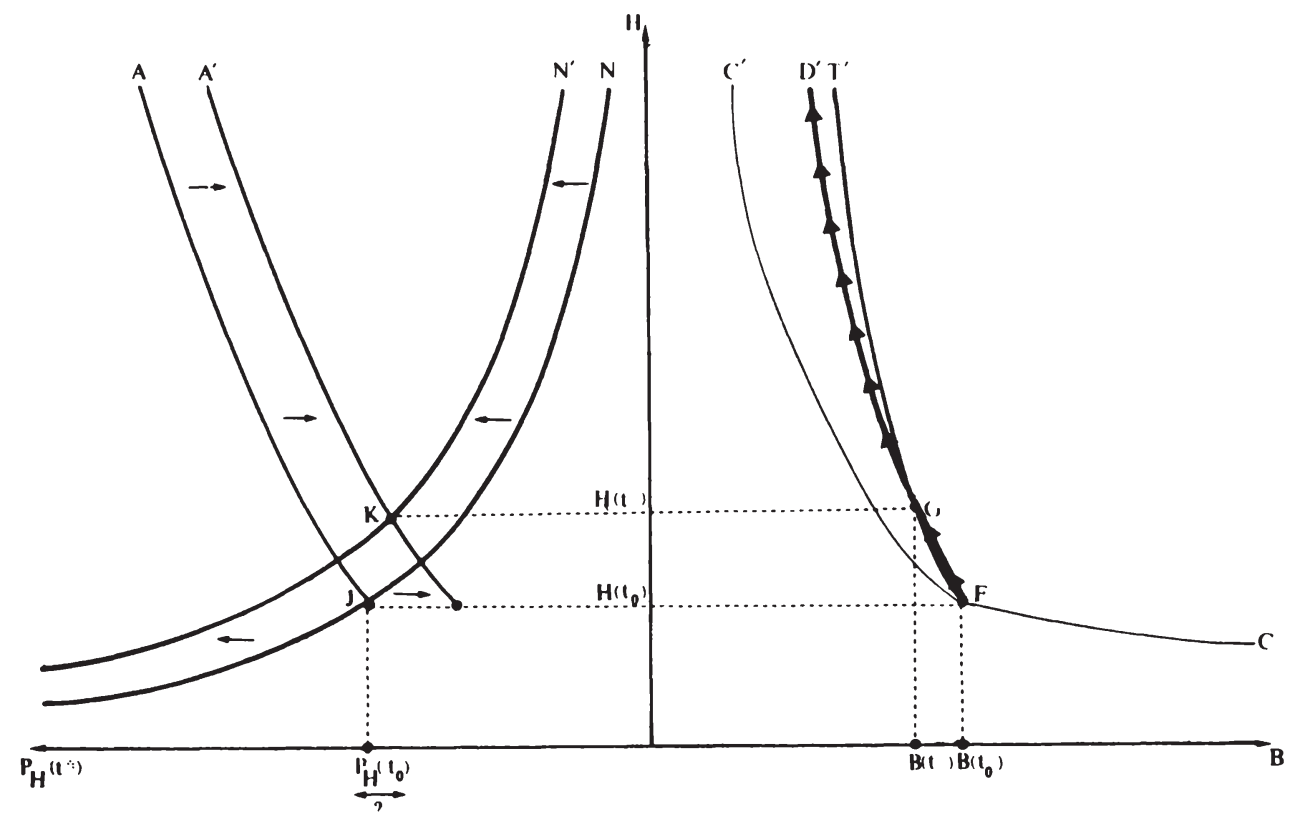

Wie man Abb. 16 entnehmen kann, wirkt das gegenwärtig praktizierte Verfahren der Nutzungswertpauschalierung kurzfristig eindeutig stimulierend auf das Neubaugeschehen im Wohnungssektor ein. Ausgehend von der Gleichgewichtssituation $J$ hat die Einführung einer Steuer auf den gemäB \$21a EStG ermittelten Nutzungswert der Wohnung einen vorübergehenden Neubauboom zur Folge, in dessen Verlauf der Wohnflächenbestand (das Wohnleistungsangebot) von $H\left(t_{0}\right)$ auf $H\left(t^{*}\right)$ springt. Nach AbschluB der Kunstzeitphase $\left(t_{0^{\prime}} t^{*}\right)$ entwickelt sich das system entlang des Pfades GD' weiter: da die Besteuerung nach \$21a EStG keinen 
EinfluB hat auf die modellendogenen wachstumsraten ${ }^{92}$, ist

- der Wohnflächenbestand zu jedem zeitpunkt jenseits von $t$ * größer,

- der Baulandbestand zu jedem zeitpunkt jenseits von t' geringer, als dies ohne Besteuerung fiktiver Mieteinnahmen der Fall gewesen wäre.

- Dauerhaft angehoben werden auch der Zeitpfad

- der Kapitalintensität der Bebauung und

o des Bodenpreises - der Wohnungsinvestor muß sich die Bereitschaft des Bodenmaklers, innerhalb der Kunstzeitphase zusätzliches Bauland bereitzustellen, durch die Entrichtung eines höheren Bodenpreises erkaufen; rach $t$ * wächst $P_{B}$ von der Einkommensbesteuerung des Wohnungsinvestors unbeeinflubt weiter.

- Dauerhaft sinken wird dagegen das Niveau der Wohnungsmieten. Bei unverändertem zeitpfad der Wohnleistungsnachfrage sind die Wohnungsinvestoren nur dann in der Lage, ihr zusätzliches Angebot an Wohnleistungen am Markt unterzubringen, wenn sie bereit sind, einen geringeren Preis für diese Leistungen $z u$ akzeptieren.

All diese Ergebnisse gelten wohlgemerkt nur für den Fall, in dem der Wohnungsinvestor über genügend Eigenkapital verfügt, um seine Investitionen auch optimal finanzieren zu können.

Haben wir es stattdessen mit einem weniger begüterten selbstnutzer zu tun, der diesen optimalen Finanzierungsweg nicht beschreiten kann, so ist zu vermuten, daß sich die oben beschriebenen expansiven Effekte zumindest abschwächen. Da der "arme" Wohnungsinvestor im Grenzfall gezwungen ist, seine gesamten Ausgaben (abzüglich der öffentlichen Subventionen) für Reno-

92) Aus (III. 148) folgt $\hat{\mathrm{P}}_{\mathrm{H}}=\beta \hat{\varepsilon}$, aus (III.149) folgt $\hat{\mathrm{P}}_{B}=\hat{\varepsilon}_{\text {; darüberhinaus }}$ gelten auch weiterhin die Bedingungen (II.69) und (II.74). 
vierungsarbeiten und Neubauinvestitionen mit Krediten zu finanzieren, hat er höhere effektive Finanzierungskosten zu tragen als sein "reiches" Pendant; denn Fremdkapitalzinsen können infolge des Verzichts auf die Besteuerung fiktiver Mieteinnahmen nicht mehr steuermindernd geltend gemacht werden.

Um zu sehen, wie der "arme" Selbstnutzer auf die Pauschalbesteuerung nach \$21a EStG reagiert, gehen wir wiederum aus von den allgemeinen Bedingungen (II.23), (II.28), (II.29) und (II.30). In Gegensatz zum Planungsproblem des selbstfinanziers sind die partiellen Differentiale aus (III.146) im Planungsproblem des kreditfinanzierenden Selbstnutzers nicht mehr unbedingt gleich Null. Wie wir aus der Analyse der LaissezFaire- రkonomie wissen, sind Kreditinstitute bereit, Darlehen maximal in Höhe des Wertes der Nettoinvestition zu$z$ üglich der eingetretenen Wertsteigerung des Wohnflächenbestandes $\mathrm{zu}$ vergeben. Die summe $\mathrm{P}_{\mathrm{H}} \dot{\mathrm{H}}+\dot{\mathrm{P}}_{\mathrm{H}} \mathrm{H}$ kennzeichnet demnach das dem Wohnungsinvestor maximal zur Verfügung stehende Kreditvolumen einer Periode. Da dieser sich aber darüber im klaren ist, daß die Kreditfinanzierung die für ihn teuerste Form der Finanzierung darstellt, wird er diesen spielraum nicht voll ausschöpfen, sondern nur jenen Teil der Ausgaben fưr Bauleistungen, Bauland und Renovierungsarbeiten mit Krediten finanzieren, der nicht durch öffentliche subventionen gedeckt ist. Ein Wohnungsinvestor, der über sonstiges Elgenkapital nicht verfügen kann, ist demzufolge gezwungen, sich in jeder periode in Höhe von

$$
\begin{aligned}
S= & F^{d} \varepsilon+P_{B} F^{d}+z E-\tau_{e} \rho_{3} F^{d} \\
& -\tau_{e}\left[\gamma_{2} \rho_{1}+\left(1-\gamma_{2}\right) \rho_{2}\right]\left(F^{d} \varepsilon+P_{B} F^{d}\right)
\end{aligned}
$$

D-Mark netto neuzuverschulden.

Aus (III.154) errechnet man leicht die folgenden partiellen 
Ableitungen:

$$
\begin{aligned}
& \text { (III.155) } \frac{\partial S}{\partial E}= z, \\
& \text { (III.156) } \frac{\partial S}{\partial \varepsilon}= 1-\tau_{e}\left[\gamma_{2} \rho_{1}+\left(1-\gamma_{2}\right) \rho_{2}+\rho_{3}\right] F^{d}, \\
& \text { III.157) } \frac{\partial S}{\partial F} d=1-\tau_{e}\left[\gamma_{2} \rho_{1}+\left(1-\gamma_{2}\right) \rho_{2}+\rho_{3}\right] \varepsilon \\
&-\tau_{e}\left[\gamma_{2} \rho_{1}+\left(1-\gamma_{2}\right) \rho_{2}\right] P_{B^{\prime}}
\end{aligned}
$$

III.158) $\quad \frac{\partial S}{\partial H}=0$.

Da wegen (III.144) und (III.145)

(III. 159)

$$
\frac{\partial H}{\partial S}=-\frac{\tau_{e}}{\Theta_{e}}
$$

gilt, erhalten wir unter Berücksichtigung der partiellen Differentiale aus (III.155) bis (III.159) die folgenden, für den optimalen Reinvestitions- und Neubauplan des "armen" selbstnutzenden Wohnungsinvestors notwendigen Optimalitätsbedingungen:

$$
\begin{aligned}
& \text { (III. 160) } \quad \mathrm{P}_{\mathrm{H}}\left\{\begin{array}{l}
< \\
\geq
\end{array}\right\} \frac{z}{\theta_{\mathrm{e}}} \Rightarrow \mathrm{E}\left\{\begin{array}{l}
=0 \\
=\alpha \delta \mathrm{H},
\end{array}\right. \\
& \text { (III. 161) } \quad P_{H^{\prime}} \varphi^{\prime}(\varepsilon)=\frac{1-\tau_{e}\left[\gamma_{2} \rho_{1}+\left(1-\gamma_{2}\right) \rho_{2}+\rho_{3}\right]}{\Theta_{e}} \text { ， } \\
& \text { (III. 162) } \quad \frac{\beta}{\alpha} \varepsilon\left(1-\frac{\tau e^{\rho_{3}}}{\tau_{e}\left[\gamma_{2} \rho_{1}+\left(1-\gamma_{2}\right) \rho_{2}\right]}\right)\left\{\begin{array}{l}
< \\
=P_{B}
\end{array} \rightarrow^{d}\left\{\begin{array}{l}
= \\
\geq
\end{array}\right\} 0\right. \text {, } \\
& \text { (III. 163a) } \quad \hat{\mathrm{P}}_{\mathrm{H}}=0 \quad \text { für } t_{\mathrm{O}} \leq t<t ;
\end{aligned}
$$

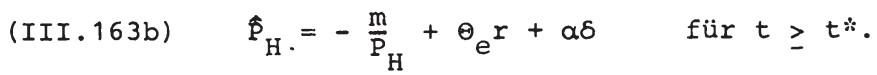


Die Bedingungen (III. 160) bis (III. 163b) bestätigen unsere eingangs geäußerte Vermutung, daß der "arme" selbstnutzer nicht im gleichen Maße von den Vergünstigungen des §21a EStG profitiert wie der "reiche" Selbstnutzer; es ist sogar der Fall denkbar (wenn nicht gar wahrscheinlich), daß die gegenwärtig praktizierte Form der Nutzungswertpauschalierung der Vermögensbildung in Form von Wohnungseigentum zum Nachteil gereicht, wenn selbstnutzende Wohnungsinvestoren gezwungen sind, mit Fremdkapital zu finanzieren:

- zunächst verteuern sich infolge der suboptimalen Finanzierung die fälligen Renovierungsarbeiten; wie aus (III.160) hervorgeht, wird es für den Wohnungsinvestor erst dann rentabel, werterhaltende Maßnahmen an seinen Gebäuden durchzuführen, wenn der Häuserpreis das im Vergleich zur Laisser-Faire-Welt höhere Niveau $\mathrm{P}_{\mathrm{H}}=1 / \hat{\mathrm{e}}$ erreicht hat. Wenn wir unterstellen, daß der Häuserpreis zum Zeitpunkt $t_{0}$ diese Grenze schon überschritten hat, kommen wir, trotz der gestiegenen Kosten, $z u$ dem Ergebnis, daß zu jedem zeitpunkt jenseits von t* die gesamten abnutzungsbedingten Substanzminderungen des Gebäudekapitalstocks durch werterhaltende Maßnahmen wiederaufgeholt werden;

- im gleichen Maße wie die Ausgaben für werterhaltende Maßnahmen steigen auch die Aufwendungen für die im Rahmen des Wohnungsneubaus benötigten Bauleistungen: das Wertgrenzprodukt des letzten beim Bau neuer Wohnungen eingesetzten Leistungsbündels entspricht nicht mehr dem für den "reichen" Selbstnutzer relevanten effektiven Preis $\left[1-\tau e\left(\gamma_{2} \rho_{1}+\left(1-\gamma_{2}\right) \rho_{2}+\rho_{3}\right]\right.$ je Leistungseinheit, sondern einem $1 / \Theta_{e}$ fach höheren [vg]. Bedingung (III.161)]. Sehr wahrscheinlich wird der verbilligende Effekt der öffentlichen Subventionen durch die kostenerhöhenden Folgen des "Zwangs" zur Kreditfinanzierung übertrcifen. Dies wird genau dann der Fall sein, wenn der Barwert der auf die letzte für Bauleistungen verausgabte D-Mark entfallenden staatlichen subventionen kleiner ist als $\tau^{\prime} e^{\prime}$ Wenn man bedenkt, $d a B\left[\gamma_{2} \rho_{1}+\left(1-\gamma_{2}\right) \rho_{2}+\rho_{3}\right]=1$ im Er- 
gebnis einer Sofortabschreibung der gesamten Anschaffungs- bzw. Herstellungskosten des Gebäudes gleichkommt, geht man sicherlich nicht $\mathrm{zu}$ weit, wenn man in (III.161) $\mathrm{P}_{\mathrm{H}} \varphi^{\prime}(\varepsilon)>1$ unterstellt:

- daß sich infolge der suboptimalen Finanzierung aber nicht nur die effektiven Kosten für Bauleistungen, sondern in gleichem Maße auch die für Bauboden erhöhen, geht aus Bedingung (III.162) hervor: nur so läßt sich erklären, daß für den "armen" Wohnungsinvestor auch in Anbetracht der gestiegenen Finanzierungskosten offensichtlich kein Anreiz besteht, bei gegebenen Bodenpreisen eine andere Kapitalintensität der Bebauung zu wählen als der "reiche" selbstnutzer;

- auf di.e Nutzungskosten des im Sektor des selbstgenutzten Wohnungseigentums gebundenen Vermögens übt die wahl der teureren Finanzierungsform dagegen keinen Einfluß aus: wie uns Bedingung (III.163b) zeigt, genießt auch der "arme" Wohnungsinvestor die Vorzüge steuerfreier Mieteinnahmen. Die Nutzungskosten der letzten von ihm in Wohnungseigentum investierten D-Mark Eigenkapital - dieses Eigenkapital setzt sich zusammen aus den Steuersubventionen und den sich im Zeitablauf kumulierenden Wertsteigerungen des Grundstückvermögens - sinken deshalb im Vergleich zur steuerfreien welt um den Betrag $\tau \mathrm{e}^{r}$. Die Ergebnisse unserer Analyse der steuerlichen Behandlung (vollständig) selbstgenutzten Wohneigentums erlauben uns die Formulierung der folgenden These:

Aufgrund der finanzierungsbedingt höheren investitionskosten wird es der "arme" Wohnungsinvestor im Vergleich zum "reichen" noch schwerer haben, selbstgenutztes worneigentum zu erwerben, als dies in einer steuerfreien welt ohnehin schon der fall ist. 
Ob die Vermögensbildung im Sektor der nicht-vermögenden Selbstnutzer durch das praktizierte Pauschalierungsverfahren im Vergleich zur Laisser-Faire-Situation beginstigt oder diskriminiert wird, läßt sich dagegen nicht eindeuttg sagen; hierzu bedürfte es exakter Informationen über das quantitatve Ausmaß des Vorteils der Nichtbesteuerung von Mieteinnahmen auf der einen Seite und des steuerinduzierten Finanzierungskostennachteils auf der anderen. Nicht zuletzt dieser Unbestimmtheit wegen können wir auf eine graphische Darstellung der Inzidenz des Pauschalierungsverfahrens verzichten, wenn selbstgenutztes Wohnungseigentum mit Fremdkapital finanziert wird; die Vielfalt in Frage kommender Ergebnisse entspräche in etwa der, die wir im Falle der Einkunftsermittlung nach $\$ 21$ EstG ermittelt haben.

Im Hinblick auf die Allokationseffizienz der in unserem Lande praktizierten Besteuerung von Einkünften aus Vermietung und Verpachtung kommen wir damit zu einigen bemerkenswerten Ergebrissen:

- keines der betrachteten Verfahren zur Ermittlung der steuerpflichtigen Mieteinkünfte vermag den Anforderungen einer effizienten Form der Einkommensbesteuerung zu genügen;

- lediglich hinsichtlich des Pauschalierungsverfahrens - und dies auch nur dann, wenn der betreffende Wohnungsinvestor optimal zu finanzieren in der Lage ist - ist ein eindeutiges Urteil über die Art der steuerinduzierten Wohlfahrtsverluste möglich; in diesem Fall wird ein im Vergleich zun Paretooptimum zu großer Anteil des volkswirtschaftlichen Vermögens in Form (selbstgenutzten) Wohnungseigentums gebunden;

- dagegen läßt sich selbst in der Partialanalyse nicht mit letzter Sicherheit sagen, ob das praktizierte Uberschusverfahren die Kapitalbindung im (Miet-)Wohnungssektor im Ver- 
gleich zur steuerfreien Situation erleichtert oder erschwert auch wenn Schlagworte wie "erhöhte AfA" oder "degressive AfA" eine steuerliche Bevorzugung des (vermietenden) Wohnungsinvestors suggerieren: $z u$ bedenken ist hierbei, daß einerseits zwar durch steuerliche AfA der Einsatz von Bauleistungen bei der Produktion von Wohnflächeneinheiten subventioniert wird und Wohnungsinvestitionen insofern begünstigt werden, daß andererseits aber die tatsächlichen Wertminderungen des Grundstückvermögens, die mit dem abnutzungsbedingten Substanzverzenr im Gebäudekapitalbestand in einem nur indirekten zusammenhang stehen, durch das geltende Uberschußverfahren ebensowenig erfaßt werden wie nachfragebedingte Wertsteigerungen dieses Vermögensbestandes. Sollte der Ertragswert des Grundstückvermögens infolge des Aufeinandertreffens dieser beiden Effekte sinken, würde dem zur Einkunftsermittlung nach \$21 EStG verpflichteten Wohnungsinvestor hieraus ein eindeutiger Vermögensnachteil erwachsen, die Rentabilität der Wohnungsinvestition insoweit also sinken. Ob der begünstigende Effekt der steuerlichen AfA diesen Nachteil aufwiegen kann, läßt sich nicht von vornherein sagen;

- nicht beantworten läßt sich deshalb auch die Frage, ob nun die UberschuBrechnung nach §21 EStG oder die Pauschalierung des Nutzungswertes nach §21a EStG das für den Wohnungsinvestor günstigere Verfahren der Einkunftsermittlung ist.

Der theoretischen Unbestimmtheit der relativen Vorteilhaftigkeit beider Methoden zur Ermittlung der Einkünfte aus Vermietung und Verpachtung steht in der Praxis eine eindeutige Präferenz für die UberschuBrechnung gegenüber. Ausschlaggebend für diese Vorliebe sind unseres Erachtens nicht hauptsächlich die für die UberschuBrechnung charakteristischen Liquiditätsvorteile, sondern zwei weitere, bisher noch nicht angesprochene Möglichkeiten, die sich dem Wohnungsinvestor infolge konzeptioneller Măngel des deutschen Einkommensteuerrechts bieten:

- die Möglichkeit des vorzeitigen Verkaufs und

- die Möglichkeit der Artenfortschreibung. 
5.2.2.2.3. überschußrechnung und (vorzeitiger) verkauf

Eine dieser Möglichkeiten besteht darin, daß der Wohnungsinvestor sein Wohnungseigentum nicht bis zu dessen ökonomischem Lebensende nutzt, sondern es schon vor diesem zeitpunkt verkauft 93) Das geltende steuerrecht enthält Regelungen, die zu einem solchen Verhalten geradezu herausfordern; es genügt schon ein einfaches Beispiel zur Begründung dieser Aussage:

Betrachten wir zwei Investoren, von denen jeder zum zeitpunkt $t_{0}$ ein bebautes Grundstück erworben hat, sei es als Käufer, sei es als Bauherr. Beide Grundstücke seien bis ins Detail identisch, sowohl was die bauliche Gestaltung als auch was die Rentabilität anbelangt. Der Herstellungs- bzw. Anschaffungskostenanteil des Gebäudes am Gesamtpreis des Grundstücks kann nach geltendem Recht beschleunigt abgeschrieben werden. Sollten sich beide Investoren nun $z u$ einem belieblgen zeitpunkt $t_{1}>t_{0}$ dazu entschließen, ihre Grundstucke einander wechselseltig zu verkaufen, so könnten sie - solange sie nicht den steuerlichen Status eines Gewerbetreibenden tragen - einen zusätzlichen, uber die subventionierung von Bauleistungen im Rahmen der steuerlichen AfA hinausgehenden Abschreibungsvorteil realisieren: denn nach Mißßgabe des bundesdeutschen Einkommensteuerrechts werden die steuerlichen Abschreibungsrestwerte im Falle der Grundstuckstransaktion nicht auf den Käufer übertragen, auch bleiben die beim Verkauf des Grundstücks aufgedeckten stillen Reserven steuerfrei. Als Basis für die vom Käufer jenseits von $t_{1}$ vorzunehmenden steuerlichen Abschreibungen dient der höhere Verkaufswert des Gebäudes; đaß der Verkaufswert des Gebäudes tatsächlich über

93 ) Daß dies selbst in einer welt ohne Steuer ökonomisch rational sein kann, wissen wir aus der betriebswirtschaftlichen Investitionstheorie. Die Bestimmungsgruinde hierfir sollen an dieser Stelle jedoch nicht erbrtert werden; - vgl. hierzu SCHNEIDER, D. (1974), S.282 ff. 
dessen Buchwert liegt, folgt

- zum einen aus der Tatsache, daß der Erhaltungsaufwand in der Steuerbilanz nicht aktiviert wird, obgleich er den Ertragswert des Grundstücks positiv beeinflußt,

- zum anderen aus dem Umstand, daß auch die nachfragebedingten Wertzuwächse in der Steuerbilanz keinen Niederschlag finden.

Vom ökonomischen standpunkt aus betrachtet haben wir es bei der betrachteten Wohnimmobilie folglich mit zwei verschiedenen Anlagegütern $z u$ tun - mit einem, das Wohnleistungen produziert und auf diesem Wege Mieteinnahmen und Wertzuwächse erwirtschaftet und einem, durch dessen "Einsatz" sich steuerliche AfA-Vergünstigungen erzielen lassen. Beurteilt man die obige Transaktion unter diesem Aspekt, so kommt der wechselseitige Verkauf praktisch einem kostenlosen Austausch des zweiten Anlagegutes gegen ein artgleiches, aber "produktiveres" gleich: durch den Verkauf stellen sich beide Investoren rentabilitätsmäßig so, als würden sie ihr Wohneigentum bis $z$ u dessen ökonomischem Lebensende nutzen, wobei ihnen durch das Steuergesetz aber die Möglichkeit eingeräumt wird, zum zeitpunkt $t_{1}$ die Abschreibungsbasis $\mathrm{zu}$ vergröBern ${ }^{94)}$.

Die formale Darstellung der eben geschilderten Problematik w.Lrd erschwert durch Unklarheiten im Gesetzestext. Wie wir wissen, erlaubt der Gesetzgeber Absetzungen für Abnutzung le-

94)Es ist offensichtlich, daß das Auftreten dieser Rentabilitätsvorteile nicht an eine bestimmte Anzahl van Investoren oder gar an den Beispielfall des wechselseitigen Verkaufs gebunden ist (der ja zudem durch $\$ 7 \mathrm{~b}$, Absatz 1, Satz 4, Ziffer 2 EStG von den Vergünstigungen der erhöhten Absetzungen ausgeschlossen ist). Voraussetzung ist allein, daß (annähernd) perfekte Märkte vorhanden sind, daß jedem Käufer eine (beschleunigte) Abschreibung auf Anschaffungskostenbasis gestattet wird und aufgedeckte stille Reserven nicht versteuert werden müssen. 
diglich für die Herstellungs- bzw. Anschaffungskosten des Gebäudes. Während die Gebäudeherstellungskosten im Regelfall leicht abzugrenzen sind - Abschreibungsbemessungsgrundlage ist dann, wie in Abschnitt III.5.2.2.2.1. dargestellt, der bilanzielle Restwert der ursprünglichen Investitionsausgaben $F^{d}\left(t_{0}\right) \varepsilon\left(t_{0}\right)$ - entstehen bei der Ermittlung des Anschaffungskostenanteils des Gebäudes am Gesamtkaufpreis des Grundstücks dann Schwierigkeiten, wenn das Grundstück längere zeit nach Fertigstellung des Gebäudes käuflich erworben wurde. Da im Falle des Grundstückkaufs regelmäßig ein Gesamtkaufpreis vereinbart wird, muß dieser, einem Urteil des Bundesfinanzhofes zufolge, zur Ermittlung der Abschreibungsbemessungsgrundlage im Verhältnis der geschätzten "Verkehrswerte" von Grund und Boden einer-, seits und aufstehendem Gebäude andererseits aufgeteilt werden. offensichtlich geht der BFH davon aus, daß der "Verkehrswert" des Gebäudes losgelöst vom Wert des Grund und Bodens als solcher existiert; solange Gebäude aber nicht mobil sind, läßt sich diese unterstellung unter ökonomischen Gesichtspunkten nicht rechtfertigen.

Für die Zwecke unserer Analyse empfiehlt es sich daher, auf eine idealisierte Form der Schätzung des Anschaffungskostenanteils des Gebăudes zurückzugreifen, die zugleich wohl auch den Willen der Finanzgerichtsbarkeit widerspiegeln dürfte: wie wir aus der vorangegangenen Analyse wissen, entsprechen im Gleichgewicht die Ausgaben für das Gebäude der Dj.fferenz. aus dem Marktwert des neu geschaffenen Wohnraums und den Ausgaben für das baureife Grundstück; es gilt

$$
\text { (III. 164) } \quad F_{\varepsilon}^{d}=P_{H} F^{d} \varphi(\varepsilon)-P_{B} F^{d} \text {. }
$$

Wie wir ebenfalls wissen, wächst der Preis einer jeden Wohnflächeneinheit im Zeitablauf mit der Rate $\hat{\mathrm{p}}_{H}$. Da alle abnutzungsbedingten Substanzminderungen des Gebäudes durch werterhaltende Maßnahmen ausgeglichen werden, wird sich der Marktwert des gesamten Grundstücks zum Zeitpunkt $t_{1}$ auf

95) BFH, Urteil vam 9.12.1972, BStBl. 1973II, S.295. 
(III. 165)

$$
M_{W}\left(t_{1}\right)=P_{H}\left(t_{0}\right) \exp \left[\hat{P}_{H}\left(t_{1}-t_{0}\right)\right] F^{d}\left(t_{0}\right) \varphi\left[\varepsilon\left(t_{0}\right)\right]
$$

D-Mark belaufen, wenn die Baufertigstellung zum zeitpunkt $t_{0}$ erfolgte. Es ist plausibel, das oben zitierte Urteil des Bundesfinanzhofes dahingehend $z u$ interpretieren, daß Gebäuảekapital und Grund und Boden von der Wertsteigerung des gesamten Grundstücks gleichermaßen profitieren. Akzeptiert man diese Interpretation, so erhält man zum zeitpunkt $t_{1}$ einen geschätzten "Gebäudewert" $\mathrm{G}$ in Höhe von

$$
G\left(t_{1}\right)=F^{d}\left(t_{0}\right) \varepsilon\left(t_{0}\right) \exp \left[\hat{P}_{H I}\left(t_{1}-t_{0}\right)\right]
$$

(III.166) erlaubt uns nun, die allokativen Konsequenzen eines vorzeitigen Verkaufs auch analytisch aufzuzeigen. Betrachten wir hierzu zunächst die zeitliche Entwicklung des steuerlichen Buchwertes eines zum zeitpunkt $t_{0}$ errichteten Gebäudes. Die Herstellungskosten dieses Gebäudes betragen $F^{d}\left(t_{0}\right) \varepsilon\left(t_{0}\right)$. Dieser Betrag kann in jährlichen Beträgen mit der konstanten Rate $\mu$ beschleunigt abgeschrieben werden. Da Erhaltungsaufwand nicht aktivierungsfähig ist, erhalten wir für den Restbuchwert $R$ dieses Gebäudes zum Zeitpunkt $t_{1}$ den D-Mark-Betrag

$$
R\left(t_{1}\right)=F^{d}\left(t_{0}\right) \varepsilon\left(t_{0}\right) \exp \left[-\mu\left(t_{1}-t_{0}\right)\right]
$$

Verkauft der Wohnungsinvestor das Grundstück nun zum Zeitpunkt $t, z u m$ herrschenden Marktpreis, und verwendet er den Verkaufserlös für den Erwerb eines identischen objekts, so stimmt der steuerliche Buchwert des neuen Grundstücks mit dem Gebäudewert $G\left(t_{1}\right)$ aus (III.166) uberein; offensichtlich gilt $R\left(t_{1}\right)<G\left(t_{1}\right)$.

Diese Betrachtung ist nicht nur auf eine Investition beschränkt, sie läBt sich auf alle Investitionen des repräsentativen Wohnungsunternehmers ausdehnen. Dazu wollen wir unterstellen, daB jede Wohnflächeneinheit nur einmal, und 2 war $\mathrm{T}_{1}=$ $t_{1}-t_{0}$ Perioden nach Fertigstellung des Gebäudes, verkauft 
wird.96)

Der gesamte Bestand des (Miet-)Wohnungssektors - und damit auch das Grundstilckvermögen des flir diesen sektor repräsentativen Wohnungsinvestors - setzt sich dementsprechend aus zwei Kategorien bebauter Grunästulcke zusammen - solchen, die schon einmal verkauft worden sind und solchen, denen der Verkauf noch bevorsteht. Ist $t_{0}$ wiederum startpunkt unserer Analyse, so wurden alle Grundstücke der ersten Kategorie innerhalb der Zeitspanne $\left(-\infty, t_{0}-T_{1}\right)$, alle Grundstücke der zweiten Kategorie dagegen innerhalb der Zeitspanne ( $t_{0}, t_{0}-T_{1}$ ) bebaut.

Wie lautet nun zum Zeitpunkt $t_{0}$ der Buchwert eines innerhalb des zeitraums $\left(-\infty, t_{0}-T_{1}\right)$ errichteten Gebäudes?

Wenn s der Fertigstellungszeitpunkt des Gebäudes war, so wurde der Abschreibungsrestwert dieses Gebäudes zum Zeitpunkt $s+T_{1}$ auf den wert $F^{d}(s) \varepsilon(s) \exp \left[\hat{P}_{H} T_{1}\right]$ angehoben und von dort an mit der Rate $\mu$ beschleunigt abgeschrieben ${ }^{97)}$ Der Buchwert $R_{1}\left(t_{0}\right)$ dieses Gebäudes lautet dementsprechend

(III. 168)

$$
R_{1}\left(t_{0}\right)=F^{d}(s) \varepsilon(s) \exp \left[\hat{P}_{H} T_{1}\right] \exp \left[-\mu\left(t_{0}-\left(s+T_{1}\right)\right)\right] \text {. }
$$

96) Dies dürfte vor allem bei im Rahmen von Bauherrenmodellen errichteten Wohnungen regelmåBig der Fall sein.

97) Um MiBverstăndnissen vorzubeugen, sollte darauf hingewiesen werden, daB sich die Annahme, die Abschreibungsrate $\mu$ komme vor und nach dem verkauf der Immobilie zur Anwendung, mit den geltenden gesetzlichen Bestimmungen nicht unbedingt im Einklang befindet: wenn der verkăufer die degressive AfA schon selbst in Anspruch genommen hat, besteht für den Kăufer nur noch die Möglichkeit, entweder die (im Zweifelsfalle ungünstigere) $7 \mathrm{~b}$-Abschreibung oder - soweit es sich bei dem Grundstück nicht um ein Einfamilienhaus oder ein Zweifamilienhaus oder eine Eigentumswohnung handelt oder beim Kăufer die objektbeschränkung greift - die lineare Absetzung nach 57 Abs. 4 EStG in Anspruch zu nehmen. Da die qualitativen Aussagen der folgenden Analyse unabhăngig sind von den spezifischen Unterschieden der erwähnten Abschreibungsverfahren, ist die obige Approximation aber sicherlich vertretbar. 
Dagegen beträgt der bilanzielle Restwert $R_{2}\left(t_{0}\right)$ eines Gebäudes, das zu elnem beliebigen Zeitpunkt $s$ innerhalb des zeitraums $\left(t_{0}-T_{1}, t_{0}\right)$ errichtet wurde,

$$
R_{2}\left(t_{0}\right)=F^{d}(s) E(s) \exp \left[-\mu\left(t_{0}-s\right)\right] \text {. }
$$

D-Mark.

Unter Berï.cksichtigung von (III.168) und (III.169) läBt sich der bilanzielle Kapitalstock des repräsentativen Wohnungsunternehmens zum Betrachtungszeitpunkt $t_{0}$ beschreiben als

(III.170)

$$
\begin{aligned}
K^{*}\left(t_{0}\right)= & \int_{t_{0}-T_{1}}^{t_{0}} F^{d}(s) \varepsilon(s) \exp \left[-\mu\left(t_{0}-s\right)\right] d s \\
& +\int_{-\infty}^{t_{0}-T_{1}} F^{d}(s) \varepsilon(s) \exp \left[T_{1}\left(\hat{P}_{H}+\mu\right)-\left(t_{0}-s\right)\right] d s \\
& =\int_{-\infty}^{t_{0}} F d(s) \varepsilon(s) \exp \left[-\mu\left(t_{0}-s\right)\right] d s \\
& +\int_{0}^{t_{0}-T_{1}} F^{d}(s) \varepsilon(s) \exp \left[-\mu\left(t_{0}-s\right)\right] \exp \left[T_{1}\left(\hat{P}_{H}+\mu\right)-1\right] d s . \\
& -\infty
\end{aligned}
$$

Das Integral in der dritten Zeile von (III.170) würde den Wert des bilanziellen Kapitalstocks widerspiegeln, wenn der Wohnungsinvestor zwar beschleunigt abschreiben könnte, auf den Verkauf der Wohnimmobilie aber verzichten wïrde. Das Integral in der vierten zeile bezeichnet die verkaufsbedingte Erhöhung des steuerlichen Buchwertes. Das Ausmaß der Begünstigung hängt offensichtlich davon $a b$, um wieviel der Hăuserpreis vom Fertigstellungszeitpunkt an bis zum Verkaufszeitpunkt gestiegen und um wieviel der Buchwert des aufstehenden Gebäudes während desselben Zeltraums gefallen ist; würde man in (III.170) $\hat{\mathrm{P}}_{\mathrm{H}}=\mu=0$ setzen, würde das Integral in der vierten zeile ebenfalls den Wert Null annehmen.

Differenziert man (III.170) nach dem Zeitindex $t$ zum Zeitpunkt $t=t_{0}$, so erhält man als Bewegungsgleichung für den bi- 
lanziellen Kapitalstock

$$
\left.\dot{\mathrm{K}} *=\mathrm{F}_{\varepsilon \exp \left[\mathrm{T}_{1}\right.}\left(\hat{\mathrm{P}}_{\mathrm{H}}+\mu\right)\right]-\mu \mathrm{K} * \text {. }
$$

Setzt man (III.171) anstel.le der Bewegungsgleichung (III.119) in das Planungsproblem (III.122) des vermietenden wohnungsinvestors ein, so läßt sich die zu diesem Kontrollproblem gehörende Hamiltonfunktion wie folgt schreiben:

$$
\begin{aligned}
H= & z \Theta_{e}(\mathrm{mH}-E-r D)+z \tau_{e} e^{\mu K^{*}} \\
& -\left[1-\tau_{e}\left(\gamma_{2} \rho_{1}+\left(1-\gamma_{2}\right) \rho_{2}\right)\right]\left(F^{d} \varepsilon+P_{B} F^{d}\right) \\
& +P_{H}\left[F_{\varphi(\varepsilon)}^{d}-z(\alpha \delta H-E)\right] \\
& +\lambda_{K^{*}}\left[F^{d} \varepsilon \exp \left[T_{1}\left(\hat{P}_{H}+\mu\right)\right]-z \mu K^{*}\right] \\
& +\lambda_{D} S .
\end{aligned}
$$

Auf die Finanzierungspräferenzen des Wohnungsinvestors übt die Möglichkeit, das Grundstück vorzeitig verkaufen zu können, offensichtlich keinen Einfluß aus: da Fremdkapitalzinsen abzugsfähig sind und die Erträge alternativer Anlageformen ebenfalls der Einkommensbesteuerung unterliegen, ist der Investor bezulglich der sich ihm bietenden Finanzierungsalternativen indifferent.

Ebensowenig beeinflußt wird der zeitpfad des optimalen Erhaltungsaufwandes: abnutzungsbedingte substanzminderungen werden, da der Häuserpreis $P_{H}$ annahmegemäß den Wert von $\Theta_{e} D$-Mark $z u$ jedem zeitpunkt jenseits von $t_{0}$ übertrifft (und deshalb $z u$ jedem dieser Zeitpunkte $y=1 \mathrm{gilt}$ ), in vollem Umfang durch entsprechende Arbeiten kompensiert.

Auf eine explizite mathematische Darstellung dieser Ergebnisse soll an dieser stelle verzichtet werden. Der Leser wird auf die entsprechenden Berechnungen in Abschnitt III.5.2.2.2.1. verwiesen. 
Interessant ist es aber, zu untersuchen, wie sich die Möglichkeit des vorzeitigen Verkaufs auf den Neubauplian des Wohnungsinvestors auswirkt. Differenziert man (III.172) nach den Kontrollvariablen $\varepsilon$ und $F^{d}$, so erhält man als notwendige Bedingungen für ein Gleichgewicht

(III.173)

$$
\begin{aligned}
& \mathrm{P}_{\mathrm{H}} \varphi^{\prime}(\varepsilon)=1-\tau_{\mathrm{e}}\left[\gamma_{2} \rho_{1}+\left(1-\gamma_{2}\right) \rho_{2}\right]-\lambda_{K^{*}} \exp \left[\mathrm{T}_{1}\left(\hat{\mathrm{P}}_{\mathrm{H}}+\mu\right)\right], \\
& \frac{\beta}{\alpha^{\varepsilon}}\left(1-\frac{\lambda_{K^{*}} \exp \left[T_{1}\left(\hat{P}_{H}+\mu\right)\right]}{1-\left[\tau_{e}\left(\gamma_{2} \rho_{1}+\left(1-\gamma_{2}\right)\right) \rho_{2}\right]}\right)\left\{\begin{array}{l}
< \\
=
\end{array}\right\} P_{B} \Rightarrow F^{d}\left\{\begin{array}{l}
= \\
\geq
\end{array}\right\} 0 .
\end{aligned}
$$

(III.174)

Aus (III.173) geht hervor, daß als Folge der Nichtbesteuerung realisierter Veräußerungsgewinne der effektive Preis für Bauleistungen sinkt. Dieses Ergebnis überrascht uns angesichts der an den Verkauf des Grundstücks geknüpften zusätzlichen $\mathrm{Ab}-$ schreibungsvergünstigungen nicht; bezogen auf jede in das Gebäude investierte D-Mark kann der Wohnungsinvestor verkaufsbedingt zusätzliche $\left\{\exp \left[\mathrm{T}_{1}\left(\hat{\mathrm{P}}_{\mathrm{H}}+\mu\right)\right]-1\right\}$ D-Mark im Rahmen der Absetzungen für Abnutzung steuermindernd geltend machen. Der Barwert der auf diesem Wege zusätzlich gevährten Steuererstattungen beträgt - wenn wir uns an die Definition des Schattenpreises $\lambda_{K^{*}}$ erinnern - $\lambda_{K^{*}}\left[\exp T_{1}\left(\hat{P}_{H}+\mu\right)-1\right]$ D-Mark. Subtrahiert man diesen Betrag von den effektiven Bauleistungskosten aus Bedingung (III.125), erhält man als Ergebnis die in Bedingung (III.173) ausgewiesenen effektiven Aufwendungen für die marginale Bauleistungseinheit.

Da die Ausgaben für Bauland von der auf den Verkauf folgenden Anhebung der steuerlichen Buchwerte ebensowenig berührt werden wie von der steuerlichen AfA überhaupt, ist die oben beschriebene verkaufsbedingte Verbilligung des Gebäudekapitals gleichbedeutend mit einer zusätzlichen Diskriminierung des Einsatzes von Bauland bei der Produktion von Wohnflächeneinheiten. Dies geht auch aus Bedingung (III.174) hervor: wenn der Bodenmakler nicht bereit ist, einen im Vergleich zur LaisserFaire-Situation geringeren Preis für jede von ihm angebotene 
Flächeneinheit Bauland zu akzeptieren, wird der Wohnungsinvestor seinerseits mit einer Einschränkung sej.ner. Baulandnachfrage und einer Erhöhung der Kapitalintensität der Bebauung der von ihm erworbenen Grundstücke reagieren. Und diese Reaktion wird dies geht aus einem Vergleich der Bedingungen (III.126)' und (III.174) hervor - stärker ausfallen als in einer situation, in der ein Verkauf des bebauten Grundstücks nicht geplant wird.

An den Relationen der modellendogenen Wachstumsraten zueinander ändert sich durch die Berücksichtigung der Verkaufsmöglichkeiten nichts. Aus (III.173) und (III.174) folgen die auch für das Paretooptimum charakteristischen Beziehungen

(III.175) $\quad \hat{\mathrm{P}}_{\mathrm{H}}=\beta \hat{\varepsilon}$,

bzw.

(III.176) $\quad \hat{\mathrm{P}}_{\mathrm{B}}=\hat{\varepsilon}$.

Aus diesem Grunde läßt sich die Entwicklung des Wohnflächenbestandes $\mathrm{H}$ und des baureifen Grundstückbestandes B durch die den Planungskalkül des nichtverkaufenden Vermieters beschreibenden isoelastischen Entwicklungspfade aus Abb.15 darstellen. Die verkaufsbedingte Verbilligung der Bauleistungskosten und die dadurch ausgelöste Erhöhung der Neubaubereitschaft des repräsentativen Wohnungsinvestors schlägt sich in einer Rechtsverschiebung der Angebotskurve $A^{\prime}$ aus Abb.15 nieder.

Dagegen behält die Nachfragekurve die ihr in Abb.15 zugewiesene Position $N^{\prime}$ bei: leitet man die Hamiltonfunktion aus (III. 172) nach der Zustandsvariablen $\mathrm{H} a b$, so erhält man auch für den Fall des zwischenzeitlichen Verkaufs die Bedingungen
(III.177a)
$\hat{\mathrm{P}}_{\mathrm{H}}=\mathrm{O}$
für $t_{0} \leq t<t^{*}$,
(III. 177b)
$\hat{\mathrm{P}}_{\mathrm{H}}=-\frac{\Theta_{\mathrm{e}}^{\mathrm{m}}}{\mathrm{P}_{\mathrm{H}}}+\Theta_{e^{r}+\alpha \delta}$ für $t \geq t *$. 
Die eindeutige Rechtsverschiebung der Angebotskurve erlaubt uns, die allokativen Konsequenzen abzugrenzen, die dem (Miet-) Wohnungssektor aus der Nichtbesteuerung von Veräußerungsgewinnen erwachsen: bestand die ursprüngliche Reaktion des Wohnungsinvestors auf die Einführung der Einkommensbesteuerung nach $\$ 21$ EStG in einem vorübergehenden Verzicht auf den Bau neuer Wohnungen, so verkürzt sich diese stagnationsphase bei Berucksichtigung von Verkaufsmöglichkeiten. Erweisen sich die in die Uberschußrechnung elngewobenen Abschreibungsvergünstigungen der Siebenerreihe des Einkommensteuergesetzes für den vermietenden Wohnungsinvestor dagegen als so vorteilhaft, daß er bereit ist, im Anschluß an die Einführung der steuer seine Wohnungsneubautätigkeit zu intensivieren, so wird sich diese Bereitschaft angesichts der mit einem vorzeitigen Verkauf des Grundstücks verbundenen Vermögensvorteile noch verstärken.

\subsubsection{4. überschurrechnung und Nutzungswechsel}

Wie wir im vorangegangenen Kapitel gesehen haben, gelingt es dem vermietenden Wohnungsinvestor durch den Verkauf seines Nohnungseigentums, jene Liquiditätsvorteile in reale Vermögensvorteile umzuwandeln, die die Uberschußrechnung gegenüber der Nutzungswertpauschalierung in der Anfangsphase der Wohnungsnutzung bietet und die auf den unbeschränkten Schuldzinsenabzug und die nach oben hin nicht begrenzte degressive AfA des $\S 7$, Absatz 5 EStG zurückzuführen sind.

$\mathrm{Ob}$ der selbstnutzende Wohnungsinvestor dieselben Vorteile zu realisieren in der lage ist, ist fraglich. Tatsache dürfte sein, daß selbstnutzende Wohnungsinvestoren den späteren Verkauf ihres Wohnungseigentums nur in den seltensten Fällen in ihren Investitionskalkül miteinbeziehen: die Motivationslage beim Eigentumserwerb ist für den selbstnutzenden Wohnungseigentümer in wesentlichen Punkten eine grundsätzlich andere als die des vermietenden Wohnungsinvestors, seine Investitionsentscheidung wird sich im Gegensatz zu der des Vermieters nicht ausschließlich auf den Rentabilitätsaspekt zurückführen lassen. 
Diese anderen, schwer objektivierbaren Bestimmungsgründe sind für jene in vielen Fällen prohibitiv hohen Transaktionskosten verantwortlich, an denen ein vorzeitiger Verkauf des selbstgenutzten Wohnungseigentums scheitern wird.

Damit verwehrt sich der selbstnutzende Wohnungsinvestor die mit dem Verkauf der Wohnimmobilie verbundenen Rentabilitätsvorteile praktisch selbst. Allerdings bietet sich ihmi eine andere Gestaltungsmöglichkeit, um in den Genuß zusätzlicher Vermögensvorteile zu gelangen; diese Möglichkeit muß als zweite Ursache für die in der Realität beobachtbare Präferenz für die Uberschußrechnung angesehen werden.

Bis Ende 1981 war die Ermittlung der Einkünfte aus Vermietung und Verpachtung an die Bestimmungen des Bewertungsrechts gebunden: befand sich die selbstgenutzte wohnung in einem Einfamilienhaus (im Sinne des Bewertungsrechts), kam die pauschale Schätzung des Nutzungswertes zum Zuge; befand sie sich stattdessen in einem aus bewertungsrechtlicher sicht andersartigen Wohngebäude, so war vom Mietwert der Wohnung auszugehen. Insbesodere war es für die Anwendung der Uberschußrechnung nicht von Bedeutung, ob das Gebäude vollständig selbstgenutzt wurde oder zumindest teilweise Mietzwecken diente.

Auf die Liquiditätsvorteile, die die Uberschußrechnung dem Wohnungsinvestor in der Anfangsphase der Wohnungsnutzung im Vergleich zum praktizierten Pauschalierungsverfahren bietet, wurde in den vorangegangenen Abschnitten hingewiesen: vor allem in dieser frühen Phase werden sich aufgrund der vollständigen Abzugsfähigkeit der Fremdkapitalzinsen und der in der Höle nicht begrenzten Absetzbarkeit der steuerlichen AfA steuerwirksame Verluste realisieren lassen, die uber die im Rahmen der Pauschalierung erzielbaren hinausgehen. Dieser Liquiditätsvorteil schwächt sich aufgrund des stetig abnehmenden Buchwertes des einzelnen Gebăudes und der fortschreitenden Entschuldung im Laufe der zeit immer mehr ab und schlăgt spätestens dann in einen Liquiditätsnachteil um, wenn die $z u$ versteuernden Einkünfte aus Vermietung und Verpachtung positiv werden. Ab diesem Zeitpunkt wäre das Pauschalierungsverfahren, 
das nach Auslaufen der 7b-Vergünstigungen Einkünfte aus Vermietung und Verpachtung in Höhe von 0 D-Mark unterstellt, zweifellos das für den Wohnungsinvestor günstigere Verfahren der Einkunftsermittlung.

Das bundesdeutsche Einkommensteuerrecht bietet dem selbstnutzenden Wohnungsinvestor die Möglichkeit, die Vortelle beider Verfahren ausnutzen zu können, ohne deren Nachteile in Kauf nehmen zu müssen. Nach altem Recht bot sich diese Möglichkeit in Gestalt der sogenannten Artenfortschreibung, die es dem Wohnungsinvestor ermöglichte, zu dem für ihn günstigsten zeitpunkt vom Uberschußverfahren nach $\$ 21$ EStG auf die pauschale Schätzung seiner Mieteinkünfte nach \$21a EStG überzuwechseln. Voraussetzung für die bewertungsrechtliche Artenfortschreibung ist eine bauliche Ungestaltung des Zweifamilienhauses (an das das Uberschußverfahren gebunden war) in ein Einfamilienhaus (auf das sich wiederum die Anwendung des Pauschalierungsverfahrens beschränkte).

Eine ausführliche mathematische Analyse des eben geschilderten Sachverhaltes erubrigt sich. Wie man leicht erkennt, werden die in Abschnitt III.5.2.2.2.2. aufgezeigten, die Vermögensbildung im Wohnungssektor begünstigenden Effekte des §21a EStG durch die Möglichkeit der Artenfortschreibung noch verstärkt. Aus diesem Grunde darf es auch nicht überraschen, daß selbstnutzende Wohnungsinvestoren in der Vergangenheit den ihnen gebotenen Gestaltungsspielraum ausgiebig genutzt haben. So läßt sich anhand der Baugenehmigungsstatistik belegen, daß der Bau des "klassischen" Eigenheims in den zurückliegenden Jahren eindeutig rückläufig war ${ }^{97}$ ), während der Anteil von Zweifamilienhäusern an der Gesamtzahl der Genehmigungen zugenommen hat. Daß sich diese Verschiebung in der struktur der Baugenehmigungen vor allem auch mit der dargelegten steuerbedingten Attraktivität von sogenannten "unechten Zweifamilienhäusern" ("unecht" deshalb, weil sie vom Wohnungsinvestor vollständig 97) HOFFMANN (1981), S.9. 
selbst genutzt werden) erklären läBt, läBt sich letztendlich zwar nicht beweisen - der Verdacht ist allerdings begründet.

Offensichtlich glaubte auch der Gesetzgeber, diesen Zustand auf Dauer nicht länger hinnehmen zu können. Einen ersten Schritt hin in Richtung auf eine grundlegende Reform der steuerlichen Behandlung des selbstgenutzten Wohneigentums sollte die im Rahmen des Zweiten Haushaltsstrukturgesetzes formulierte Loslösung der Nutzungswertpauschalierung vom Bewertungsrecht sein. Danach muß der Nutzungswert der Wohnung nicht nur beim selbstgenutzten Einfamilienhaus, sondern bei allen vollständig selbstgenutzten Wohnhäusern pauschal geschätzt werden, gleichgültig, ob diese bewertungsrechtlich als Einfamilienhaus, Zweifamilienhaus oder Mehrfamilienhaus einzustufen sind. Wird das Zwei- oder Mehrfamilienhaus dagegen teilweise vermietet, ist auch für die selbstgenutzte Wohnung als Nutzungswert der an den Erträgen der vermieteten Wohnung(en) ausgerichtete Mietwert anzusetzen.

Nach dem Willen des Gesetzgebers soll diese Neuregelung da$\mathrm{zu}$ beitragen, steuerliche Begünstigungen abzubauen, die wegen "hoher Mitnehmereffekte" und der durch sie ausgelösten "Ungleichmäßigkeiten in der Besteuerung" wohnungsbaupolitische zielsetzungen nicht in ausreichendem Maße unterstützen. ${ }^{9}$ )

Genaugenommen können damit nur die durch die Artenfortschreibung ausgelösten Verzerrungen gemeint sein; denn

- weder ist es gerechtfertigt, in der Uberschußrechnung nach $\S 21$ EStG und im Ansatz degressiver AfA eine wohnungsbauspezifische Begünstigung $z u$ sehen (vielmehr handelt es sich hierbei um eine in anderen Sektoren übliche Vorgehensweise bei der Einkunftsermittlung, wobei auch in anderen Sektoren beschleunigt abgeschrieben werden darf),

- noch ist es gerechtfertigt, die Inanspruchnahme von Abschrei-

98) "Entwurf eines Gesetzes zur Stärkung der Investitionsbereitschaft im Baubereich und zum Abbau umgleichmäßiger Besteuerung in der Wohnungswirtschaft", BT-Drucksache 9/843 vam 20.9.1981. 
bungsvergünstigungen und den Abzug von Fremdkapitalzinsen als "Mitnehmereffekt" zu interpretieren - es sei denn, der Gesetzgeber geht von der Vorstellung aus, daB diejenigen Wohnungsinvestoren, die selbstzunutzendes Wohneigentum erwerben, diesen Erwerb unter Vernachlässigung aller Rentabilitätsüberlegungen durchführen. Sollte dies der Fall sein, müßte man konsequenterweise die Effektivität der staatlichen Maßnahmen zur Förderung der Wohnungseigentumbildung in ihrer Gesamtheit in Frage stellen.

Doch gerade die aus der Artenfortschreibung resultierenden Fehlentwicklungen werden durch die Novelle nicht korrigiert. Denn an keiner Stelle des Einkommensteuergesetzes in der Fassung vom 6.12.1981 wird gesagt, wie die Ermittlung des Nutzungswertes der selbstgenutzten Wohnung im Anschluß an eine Nutzungsänderung des Gebäudes aussehen soll; hieraus darf man zweierlei schließen:

- dem Steuerpflichtigen ist es auch künftig völlig freigestellt, die Nutzung seines Wohnungseigentums $z u$ verändern (durch den Abschluß von Zeltmietverträgen läßt sich dieser Nutzungswechsel zudem zeitlich optimal planen), d.h. von der (Teil-) Vermletung auf die (völlige) Selbstnutzung zu wechseln;

- parallel zu diesem Nutzungswechsel wird auch weiterhin eine Anderung in der Nutzungswertermittlung einhergehen (die Sechs-Monats-Frist des $\$ 21 \mathrm{a}$, Absatz 1, Satz 3, Ziffer 2 EStG einmal vernächlässigt).

Durchaus möglich ist es, daß der Gesetzgeber den Wechsel in der Nutzungswertermittlung im zuge des zweiten Haushaltsstrukturgesetzes sogar erleichtert hat, ist doch nun ein Umbau des Zweifamilienhaus in ein Einfamilienhaus ebensowenig erforderlich wie eine Artenfortschreibung, die ein umständliches Verwaltungs- 
verfahren voraussetzt. ${ }^{99)}$

Darüber hinaus besteht für den selbstnutzenden Wohnungsinvestor sicherlich auch ein Anreiz, Scheinmietverhältnisse einzugehen, um die Vorteile der Uberschußrechnung in der Anfangsphase der Nutzung ausnutzen zu können. Nur eine permanente Wohnungsnutzungskontrolle könnte die Durchsetzung des Gesetzgeberwillens garantieren. Die Erfahrung zeigt aber, daß die Finanzverwaltung mit der Durchführung dieser Aufgaben überfordert ist. ${ }^{100)}$

Offensichtlich sind die im Zweiten Haushaltsstrukturgesetz enthaltenen Neuregelungen wenig dazu geeignet, das gesteckte Ziel einer gleichmäßigeren Besteuerung verwirklichen zu helfen. Vielmehr haben es die wohnungspolitisch Verantwortlichen versäumt, das Ubel an seiner Wurzel zu packen und das Nebeneinander zweier methodisch sehr unterschiedlicher Verfahren zur Ermittlung von Einkünften aus Vermietung und Verpachtung abzuschaffen.

Inzwischen scheinen sich aber alle im Bundestag vertretenen Parteien darüber einig zu sein, daß die Ertragsbesteuerung des selbstnutzenden Wohnungsinvestors einer grundlegenderen Neuordnung bedarf. Uberhaupt nicht mehr einig ist man sich dagegen uber die Art und Weise, wie diese Neuregelung en détail durchgeführt werden sollte.

99) Wohl unter dem Eindruck dieser Einsicht hat der Gesetzgeber mit dem §34f E'StG eine an die Pauschalbesteuerung nach \$21a EStG und die Inanspruchnahme erhöhter Absetzungen nach §7b EStG gebundene, von der Zahl der zum Haushalt des Wohnungsinvestors gehörenden Kinder abhängige zusätzliche Steuerermäßigung gewährt. Zweifellos macht diese Steuerermäßigung den Bau eines unechten Zweifamilienhauses tendenziell weniger attraktiv, da die für diese Subvention in Frage kammenden Kinder zu dem Zeitpunkt, zu dem der Ubergang auf die Pauschalierung normalerweise stattfinden würde, das elterliche Haus erfahrungsgemä $\beta$ bereits verlassen haben. Daß diese Regelung zu einem Mehr an steuerlicher Gleichbehanalung beitragen kann, darf aber bezweifelt werden. Auch stellt sich eine Wohnungspolitik, die erkannte Maingel mit solch abenteuerlichen Mitteln zu beheben versucht, ein denkbar schlechtes Zeugnis aus.

100) Aus diesem Grunde mußte beispielsweise die ursprüngliche Beschränkung des \7b EStG auf die zumindest teilweise Selbstnutzung von Einfamilienhäusern, Zweifamilienhäusern und Eigentumswohnungen aufgegeben werden. 
5.2.2.2.5. "Investitionsgutlösung" versus "Konsumgutlösung"

Dem Gesetzgeber eröffen sich bei der geplanten Reform zwei grundsätzlich verschiedene Alternativen:

- zum einen hat er die Möglichkeit, das Investitionsgut "Wohnung" einkommensteuerlich auch tatsächlich wie ein Investitionsgut zu behandeln;

- zum anderen steht es ihm offen, dem Investitionsgut "Wohnung" den einkommensteuerlichen Status eines Konsumgutes zuzuweisen.

Der grundlegende Unterschied zwischen beiden Konzeptionen kommt in der Abgrenzung der steuerpflichtigen Einkünfte aus Vermietung und Verpachtung zum Ausdruck.

Die Investitionsgutlösung in ihrer idealtypischen Form hätte zur Folge, daß

- die zu versteuernden (fiktiven) Mieteinnahmen im Rahmen einer UberschuBrechnung ermittelt und mit demselben Steuersatz belastet werden müßten wie andere Einkunftsarten auch; Bedingung hierbei wäre,

- daß die effektiv gezahlten Fremakapitalzinsen von der Steuerbemessungsgrundlage abgezogen werden dürfen und

- steuerliche Abschreibungen in jährlichen Beträgen nach dem Ertragswertverfahren gestattet werden.

Als ein mit dieser idealisierten UberschuBrechnung völlig gleichwertiges Verfahren der Einkunftsermittlung haben wir in $\mathrm{Ab}-$ schnitt III.5.2.2.2.2. die pauschale Schätzung des Mietwertes auf der Grundlage zeitnaher Einheitswerte kennengelernt.

Die Realisierung der Konsumgutlösung hätte dagegen zur Folge, daß die Eigennutzung von Wohnungseigentum für die Ermittlung der steuerpflichtigen Einkünfte ebenso irrelevant wäre wie die Nutzung des privaten PKW oder des eigenen Kühlschranks.

Daß die Investitionsgutlösung in der eben beschriebenen Form eine effiziente Lösung des angesprochenen Reformproblems darstellen wirde, haben wir in Abschnitt III.1. gezeigt. Dagegen sind die von Vertretern der wohnungspolitischen Praxis gegen eine Investitionsgutlösung vorgebrachten Bedenken häufig unuberlegt und deshalb nicht selten falsch. 
So wird vor allem von Seiten der Bausparkassen das Argument vorgebracht, daß die Investitionsgutlösung infolge des dann erlaubten Schuldzinsenabzugs für den Wohnungsinvestor einen Anreiz entstehen lasse, die Entschuldung seiner Immobilie hinauszuschieben. Dieser Vorwurf ist unbegründet: wie wir gesehen haben, garantiert gerade die Abzugsfähigkeit der Schuldzinsen, daB der Wohnungsinvestor im Rahmen einer allgemeinen Einkommensteuer keine eindeutige Präferenz für eine bestimmte Form der Finanzierung hegt. Die zusätzlichen effektiven zinskosten $\theta_{e} r$, die durch eine marginale Änderung in der Finanzierungsstruktur der Wohnungsinvestition zugunsten der Fremdfinanzierung entstehen würden, wären stets mit dem Nettozinsertrag $\theta_{\mathrm{e}} \mathrm{r}$ identisch, den eine Anlage der so freigesetzten marginalen Einheit Eigenkapital am Kapitalmarkt erwirtschaften könnte. Durch die Hinauszögerung der Entschuldung könnte der Wohnungsinvestor dem Unternehmenseigner keinen Vermögensvorteil verschaffen. 101)

Ein weiterer sehr populärer Einwand, der vielfach gegen die Investitionsgutlösung vorgebracht wird, hebt auf vermutete verteilungspolitische Mängel dieser Konzeption ab: die Investitiongutlösung sei ungerecht, weil infolge der - in unserer

101) Eine solche Hinauszögerung wird es auch dann nicht geben, wenn man von der Annahme des vollkammenen Kapitalmarktes abgeht und der Tatsache Rechnung trägt, daß der Sollzins $\mathrm{r}^{\mathrm{S}}$ in aller Regel über dem Habenzins $\mathrm{rh}^{\mathrm{h}}$ liegt. In diesem Fall wird ein ökonamisch rational handelnder Investor eine sehr starke Präferenz für die Selbstfinanzierung entwickeln: der Kosteneinsparung in Höhe von $r^{S}$, die durch die Substitution einer D-Mark Fremakapital durch eine D-Mark Eigenkapital möglich wird, stehen Opportunitätskosten in Gestalt entgangener Kapitalmarkterträge lediglich in Höhe von $\mathrm{r}^{\mathrm{h}}$ gegenüber. Es sollte klar sein, daB dieser Anreiz auch dann noch besteht, wenn zinserträge besteuert werden und Schuldzinsen als Werbungskosten abzugsfähig sind. Denn auch dann ist der Nettosollzins $(1-\tau e) r^{S}$ größer ist als der Nettohabenzins $\left(1-\tau_{e}\right) r^{h}$, so daß der Kreditnehmer auch dann durch eine Entschuldung kamparative Kostenvorteile erzielen kann. 
Analyse vernachlässigten - Progression der Einkommensteuertarifs der Reichere im Vergleich zum Ärmeren eine höhere Steuerersparnis erziele, wenn Fremdkapitalzinsen und Abschreibungen steuermindernd geltend gemacht werden dürfen.

Wer diesen Einwand vorbringt, verkennt entweder die zielsetzung der Einkommensbesteuerung oder den ökonomischen Charakter von Werbungskosten: der Fiskus beabsichtigt mit der Besteuerung des Einkommens, die ökonomische Leistungsfähigkeit des Steuerpflichtigen zu treffen; Fremdkapitalzinsen und abnutzungsbedingte wertminderungen des Grundstückvermögens sind nun aber kein Ausdruck dieser Leistungsfähigkeit, sondern Kosten, die bei dem Versuch entstehen, diese Leistungsfähigkeit $\mathrm{zu}$ erhalten oder zu steigern. Insofern stellen Werbungskosten auch kein Einkommen dar. Aus diesem Grunde muB derjenige, der befurwortet, daß der Reichere bei einer allgemeinen Einkommenserhöhung mehr zusätzliche steuern zahlt als der Ärmere, konsequenterweise auch akzeptieren, daß die steuerlast des Reicheren relativ mehr abnimmt, wenn beider Einkommen um denselben Betrag sinkt. Von Steuerersparnis zu reden, wenn Werbungskosten von der steuerbemessungsgrundlage abgezogen werden können, ist deshalb verfehlt.

Dieses Urteil muB freilich relativiert werden, wenn im Rahmen der steuerlichen AfA Beträge als Werbungskosten abgesetzt werden dürfen, die im ökonomischen Sinne keine Werbungskosten darstellen. Und tatsächlich spiegeln die Abschreibungsverfahren der Siebener-Reihe des Einkommensteuergesetzes nicht den tatsächlichen Reinvermögenszugang bzw. -abgang $\left(\dot{\mathrm{P}}_{\mathrm{H}} \mathrm{H}-\mathrm{P}_{\mathrm{H}} \alpha \delta \mathrm{H}\right)$ wider, sondern laufen im Ergebnis auf eine öffentliche Förderung des Einsatzes von Bauleistungen bei der Schaffung neuen Wohnraums hinaus. Aber selbst hier läßt sich nicht eindeutig sagen, ob der Reichere von diesen Abschreibungsvergünstigungen per saldo mehr profitiert als der Ärmere: zwar wird für ihn der Barwert der steuererstattungen tatsächlich größer sein als 
für den Ärmeren; möglicherweise wäre diese Differenz aber nocn größer gewesen, wenn er die tatsächlichen Wertminderungen hätte absetzen können.

Eine Investitionsgutlösung, die dem in Kapitel III.1. beschriebenen Idealtypus nahekommt, wäre aus wohlfahrtstheoretischer sicht sicherlich zu begrüßen. Vor allem von wissenschaftlicher seite hat man sich denn auch wiederholt für diesen Vorschlag ausgesprochen ${ }^{102)}$

Dagegen scheinen sich im politischen Raum die Befürworter einer (modifizierten) Konsumgutlösung durchgesetzt zu haben.

Voraussichtlich von 1986 an erhält das selbstgenutzte Wohnungseigentum den steuerlichen Status eines "Konsumgutes" zugesprochen, dessen Nutzung den Fiskus prinzipiell nicht interessiert. Dementsprechend wird in zukunft ein Abzug von $\mathrm{Ab}-$ schreibungen, Schuldzinsen und Erhaltungsaufwendungen bei keiner Form der Selbstnutzung - auch nicht im Falle des teilvermieteten Wohnungseigentums - mehr möglich sein.

Ob damit allerdings der "einkommensteuerlichen Begünstigung von selbstgenutztem Wohnungseigentum in ihrer bisherigen Form

102) So der Wissenschaftliche Beirat beim Bundeswirtschaftsministerium (BUNDESMINISTER DER WIRTSCHAFT (1981)), das MEADE-Kamittee(1978) und der SACHVERSTANDIGENRAT (1983) .

103) Vgl. "Leitlinien zur Neuordnung der steuerlichen Förderung des selbstgenutzten Wohneigentums", Pressemitteilungen des Bundesministers für Raumordnung, Bauwesen und Städtebau vam 4.7.1984. 
weitgehend die Grundlage" entzogen wird ${ }^{104}$, muß bezweifelt werden. Folgt man nämlich den Leitlinien des BM Bau, so ist davon auszugehen, daß dem Erwerber eines Eigenheims von der öffentlichen Händen auch weiterhin kräftig unter die Arme gegriffen wird: - eine sogenannte "Grundförderung" lehnt sich an an die bisherige Gewährung erhöhter Absetzungen; danach soll der Erwerber 8 Jahre lang einen Abzug vom zu versteuernden Einkommen vornehmen können, der 5 Prozent der Herstellungs- bzw. Anschaffungskosten ausmacht, wobei Kosten von maximal 300000 D-Mark berücksichtigungsfähig sind;

- darüberhinaus soll der Förderung in Anlehnung an den derzeitigen $\$ 34 \mathrm{f}$ EStG auch weiterhin eine familienpolitische Komponente erhalten bleiben:

- ein weiteres Förderinstrument soll auch künftig darin bestehen, daß die bisherigen Möglichkeiten, Aufwendungen in der Erwerbsphase steuerlich abzusetzen, in ihrer wirkung erhalten bleiben.

Vor allem die einfachere verwaltungstechnische Handhabbarkeit dieser Lösung und das aus ökonomischer sicht unbefriedigende Argument, daß sie im Vergleich zur Investitionsgutlösung in der Anfangsphase der Nutzung zu geringeren Steuerausfällen fuhre, sollen die Entscheidung fur die Konsumgutlösung rechtfertigen. 105), 106)

104) BUND DER STEUERZAHLER(1984), S.12.

105) Vgl. SCHNEIDER, O. (1984).

106) Daß die Konsumgutlösung verwaltungstechnisch gar nicht so einfach zu handhaben ist wie üblicherweise unterstellt wird, zeigt NACHTKAMP (1984b), S. $250 \mathrm{ff}$. 
Im Hinblick auf unseren normativen BewertungsmaBstab sind diese Argumente vordergründig. Uns muB die Frage nach der Allokationseffizienz der Konsumgutlösung interessieren. Zur Beantwortung dieser Frage bedarf es keines zusätzlichen Rechenaufwandes - es genügt ein Rückgriff auf bereits abgeleitete Ergebnisse. Es ist eine bemerkenswerte Tatsache, daB bei der Diskussion um die Reform des $\$ 21$ a EStG offenbar übersehen wird, daB die Konsumgutlösung in der Bundesrepublik Deutschland heute praktisch schon realisiert ist.

War die Pauschalierung des Nutzungswertes anfangs noch als verwaltungstechnisch vereinfachtes Äquivalent zur Uberschußrechnung gedacht, so führen die Unterbewertung des Grundstücks, der Ansatz eines zu niedrigen Multiplikators in Höhe von 1 Prozent auf den korrigierten Einheitswert sowie die Möglichkeit des zusätzlichen Werbungskostenabzugs in Höhe des Grundbetrags de facto dazu, daß die Selbstnutzung für die Zwecke der Einkommensbesteuerung irrelevant ist. Durchbrochen wird dieses Prinzip lediglich durch die Bestimmung des §21a, Absatz 3, Ziffer 2 EStG, die dem Wohnungsinvestor den Ansatz erhöhter Absetzungen erlaubt - von der Qualität her eine mit dem geplanten Sonderausgabenabzug gleichzusetzende Vergünstigung. Aufgrund dieses Sachverhalts ist es deshalb auch völlig korrekt, zur Beurteilung der Allokationseffizienz der geplanten Konsumgutlösung die in Abschnitt III.5.2.2.2.3. abgeleiteten Ergebnisse heranzuziehen.

Aufbauend auf diesen Ergebnissen muß man der Konsumgutlösung vorhalten, daB sie den Selbstfinanzier von Wohnungsinvestitionen, im Zweifelsfall also den reichen Wohnungsinvestor, begünstigt; dies ist eine Tatsache, deren Existenz sich die wohnungspolitisch Verantwortlichen unseres Landes offenbar überhaupt nicht bewußt sind. ${ }^{107}$ Selbst wenn man die damit zweifellos

107) So Bundesbauminister O. SCHNEIDER in einem Interview mit der Zeitschrift CAPITAL, Ausgabe 6/1984:"Es kommt in der Tat darauf an, eine Familie wirtschaftlich in die Lage zu versetzen, ein Bauvorhaben langfristig zu finanzieren. Ob dies mit einem Freibetrag geschieht oder durch Schuldzinsenabzug, ist doch per saldo nicht das Entscheidende." 
einhergehenden Wohlfahrtsverluste einmal vernachlässigt, so müssen doch wenigstens die mit der Konsumgutlösung verbundenen verteilungspolitischen Konsequenzen nachdenklich stimmen.

Durch die Einführung der Konsumgutlösung im Sektor des selbstgenutzten Wohneigentums ließe sich kaum eines der Probleme lösen, für die die unbefriedigende Anwendung des Pauschalierungsverfahrens verantwortlich zeichnet.

Mit der Wortschöpfung "Konsumgutlösung" wurde lediglich eine neue Bezeichnung für einen bestehenden Mißstand gefunden. An der Tatsache, daß man nach wie vor einen bedeutenden Teil der Wohnungswirtschaft in die - staatlich legalisierte Schattenwirtschaft entläßt, wird sich infolge dieser Reform nichts ändern. 
5.2.2.2.6. Der Wohnungsinvestor als gewerblicher Vermieter

Es ist ein Spezifikum des deutschen steuerrechts, daß die (einkommen-) steuerliche Belastung von Einkünften grundsätzlich davon abhängt, ob der Steuerpflichtige diese Einkünfte im Zuge einer "gewerblichen Betätigung" erzielte oder im Zuge der Verwaltung des eigenen Vermögens. Diese differenzierte Betrachtungsweise gilt auch für den Bereich der Wohnungsnutzung.

Da sich die steuerliche Behandlung des "gewerblichen" Wohnungsinvestors nicht unerheblich von der des zuvor beschriebenen nicht-gewerblichen Investors unterscheidet, empfiehlt es sich, zunächst eine Abgrenzung dessen vorzunehmen, was man unter einer "gewerblichen Betätigung" überhaupt zu verstehen hat.

Bei dem Begriff der "gewerblichen Betätigung" handelt es sich keineswegs um die Umschreibung einer mit Hilfe ökonomischer Kriterien eindeutig zu qualifizierenden Handlungsweise "gewerblich" ist vielmehr ein Prädikat, das die Finanzbehörden, wie es scheint, mehr oder weniger willkürlich verleihen.

Als Charakteristika einer gewerblichen Betätigung werden in der Literatur

- die Selbständigkeit der Betätigung,

- die Nachhaltigkeit der Betätıgung,

- die Gewinnerzielungsabsicht des Steuerpflichtigen sowie

- die Teilnahme am allgemeinen wirtschaftlichen Verkehr genannt. Darüberhinaus darf es sich bei der Vermietung nicht um eine reine Vermögensverwaltung und bei den aus der Vermietung resultierenden Erträgen nicht um Einkünfte aus Landund Forstwirtschaft oder selbständiger Arbeit handeln.

§1 der Gewerbesteuerdurchfuhrungsverordnung läßt als wichtiges Abgrenzungsproblem jedoch offen, wie der Begriff "Vermögensverwaltung" näher zu definieren ist. Ein wenig konkreter ist $\$ 14$ Abgabenordnung (AO), der einem BeschluB des Bundesfinanzhofes zufolge auch für Gewerbebetriebe Gïltigkeit besitzt: Danach liegt eine Vermögensverwaltung i.d.R. dann vor, wenn Vermögen genutzt, z.B. Kapitalvermögen verzinslich angelegt oder 
unbewegliches Vermögen vermietet wird. Die Erzielung von Einnahmen müsse in erster Linie Ausfluß des Vermögens sein, nicht dagegen Folge einer Betätigung unter Einsatz des Vermögens. Eine eindeutige Antwort auf die Frage, wann eine nicht-gewerbliche Tätigkeit in eine gewerbliche umschlägt und Mieteinkünte nicht mehr als "Einkünfte aus Vermietung und Verpachtung", sondern als "Einkünfte aus Gewerbebetrieb" zu behandeln sind, gibt $\$ 14$ AO allerdings auch nicht.

Solche durch das Gesetz gebotenen Gestaltungsspielräume werden von den Steuerpflichtigen erfahrungsgemäß intensiv genutzt, wenn sich dadurch Vorteile erzielen lassen. Daß dieses Ausnutzen von Gesetzeslücken nicht immer auch mit der ursprünglichen Intention des Gesetzgebers übereinstimmt, läßt sich eindrucksvoll am Beispiel der Diskussion um die Bauherrenmodelle belegen.

In solchen Fällen ist es dann die Aufgabe der Finanzgerichtsbarkeit und der Finanzverwaltung, Grenzen zu ziehen: so unterstellt der $\mathrm{BFH}$ eine gewerbliche Vermietung immer dann, wenn der Vermieter Sonderleistungen in einem Umfang übernimmt, der erheblich uber das bei der Vermietung von Räumen übliche Maß hinausgeht. 108 )

Eine gewerbliche Tätigkeit wird auch bei umfangreichen Veraußßerungen unterstellt, da es sich hierbei nicht mehr um Vermögensverwaltung, sondern um eine Ausnutzung der Vermögenssubstanz durch Umschichtung handle. So geht die OFD Stuttgart in ihrer Verfügung vom 15.10.1976 davon aus, daß im allgemeinen ein gewerblicher Grundstückhandel vorliegt, wenn innerhalb von 8 Jahren mehr als sechs objekte (Eigentumswohnungen oder Häuser) veräußert werden. Doch auch hier bestätigen Ausnahmen die Regel, wie beispielsweise das Urteil des Bundesfinanzhofes vom 23.2.77 zeigt.

108) Näheres dazu im Urteil des BFH van 18.1.1973, BStBl.1973II, S.561. 
Es würde zu weit führen, an dieser Stelle die wichtigsten, zum Stichwort "gewerbliche Betätigung" gefällten Urteile und ergangenen Erlasse zu diskutieren. Alle diese Urteile und Erlasse bestätigen aber den Verdacht, daB die Grenze zwischen gewerblicher und nicht-gewerblicher Tätigkeit recht unscharf ist. Hinzu kommt, daB diese Grenze von Bundesland zu Bundesland auch noch unterschiedlich weit gezogen wird. Dieser Mangel an Rechtssicherheit ist umso bedauerlicher, als es für den Wohnungsinvestor von entscheidender Bedeutung $\mathrm{zu}$ sein scheint, ob die Finanzbehörden seine Handlungsweise mit dem Prädikat "gewerblich" oder "nicht-gewerblich" belegen.

Wir wollen uns deshalb im folgenden eingehend mit dem spezifischen steuerlichen Regelungen befassen, die an den gewerblichen Status eines Steuerpflichtigen geknüpft werden.

Das Verfahren zur Ermittlung des einkommensteuerlichen Gewinns eines Gewerbebetreibenden entspricht in seiner Ausgestaltung dem in \$ 21 EStG festgeschriebenen UberschuBverfahren. Insbesondere können auch im Rahmen der "Einkünfte aus Gewerbebetrieb" die gezahlten Fremdkapitalzinsen steuermindernd geltend gemacht werden. Und auch abnutzungsbedingte Substanzminderungen im Gebäudekapital können, den Bestimmungen der siebenerReihe des Einkommensteuergesetzes folgend, von der Einkommensteuerbemessungsgrundlage abgezogen werden.

Ein wesentlicher Unterschied zwischen der einkommensteuerlichen Behandlung einer vermögensverwaltenden und einer gewerblichen Nutzung von Wohneigentum besteht in der Erfassung der beim Verkauf einer Wohnimmobilie aufgedeckten stillen Reserven. Im Gegensatz zum nichtgewerblichen Wohnungsinvestor muß der gewerbliche Vermieter realisierte Veräußerungsgewinne grundsätzlich den zu versteuernden Einkünften zuführen - es sei denn, er nimmt für sich die Ausnahmeregelung des $\$ 6 \mathrm{~b}$ EStG in Anspruch: in diesem Falle kann er nämlich die beim Verkauf freigewordenen stillen Reserven zunächst auf die Anschaffungs- bzw. Herstellungskosten für im selben Wirtschaftsjahr angeschaffte 
bzw. fertiggestellte Gebäude (inklusive Baulandkosten) übertragen 109). Die Folge dieser Ubertragung ist, das nicht die gesamten Anschaffungs- bzw. Herstellungskosten dieser Gebäude, sondern nur die um den Veröußerungsgewinn reduzierten aktiviert werden. Entsprechend kann in der Folgezeit auch nur von diesem reduzierten Buchwert abgeschrieben werden.

Es ist offensichtlich, daß es sich für den gewerblichen Investor immer lohnen wird, sich anstelle der Besteuerung seiner Veräußerungsgewinne für die erfolgsneutrale Ubertragung dieser Gewinne zu entscheiden: der Barwert der mit dieser zweiten Handlungsalternative einhergehenden AbschreibungseinbuBen ist sicherlich geringer als die zusätzliche steuerlast, die durch die Besteuerung der Buchgewinne zum Zeitpunkt ihrer Realisierung entstünde. Daß dieser Vorteil dem gewerblichen Wohnungsinvestor auch dann noch erhalten bleibt, wenn er nicht unmittelbar nach dem Verkauf ein im Sinne des Einkommensteuergesetzes geeignetes objekt findet, auf das er die stillen Reserven übertragen kann, verdankt er einer weiteren Sonderregelung des §6b EStG: denn anstelle der erfolgsneutralen Ubertragung hat er auch die Möglichkeit, in Höhe der freigesetzten stillen Reserven eine den steuerlichen Gewinn mindernde Rücklage zu bilden, um diese dann später auf neu erworbene Grundstücke zu übertragen: bis in Höhe dieser Rücklage können in den folgenden zwei Wirtschaftsjahren die Erwerbskosten von Ersatzinvestitionen in der oben beschriebenen Art und Weise gemindert werden; diese Frist verlängert sich bei neu hergestellten Gebäuden auf 4 Jahre, wenn mit ihrer Herstellung vor Ende des zweiten auf das Jahr der Rücklagenbildung folgenden Wirtschaftsjahres begonnen wurde. Die Rücklage ist dann jeweils in Höhe des abgezogenen Betrags gewinnerhöhend aufzulösen. Spätestens am Schluß des vierten Jahres nach ihrer Bildung muß sie vollständig aufgelöst sein.

109) Auf die Probleme, die bei der Zurechnung der stillen Reserven auf den "Wert" des Gebäudes und den "Wert" des Bodens auftreten können, sei hier nur am Rande hingewiesen. Wir wollen im folgenden unterstellen, daß die zurechnung auf die in Kapitel III.5.2.2.2.3. beschriebene Art und weise erfolgt. 
Zweifellos bietet die Rücklagenbildung vom Wohnungsinvestor verglichen mit der erfolgsneutralen Ubertragung von VeräuBerungsgewinnen zusätzlichen Gestaltungsspielraum, denn es steht ihm frei, innerhalb der genannten Frist den für ihn günstigsten Zeitpunkt der Auflösung $z u$ wählen. Die damit einhergehenden Vorteile treten insbesondere dann zu Tage, wenn der Wohnungsinvestor einer Einkommensteuer mit progressivem Tarifverlauf unterliegt und sein $2 u$ versteuerndes Einkommen im Zeitablauf schwankt. 110)

Doch ungeachtet dieser Gestaltungsfreiheiten ist festzuhalten, daB der gewerbliche Wohnungsinvestor infolge der Besteuerung realisierter Veräußerungsgewinne gegenüber seinem nichtgewerblichen Konkurrenten eine eindeutige Benachteiligung erfährt. Denn während der nicht-gewerbliche Wohnungsinvestor durch den vorzeitigen Verkauf seines Wohnungseigentums in den Genuß eines realen Vermögensgeschenkes kommt, wird sich für den gewerblichen Wohnungsinvestor eine ausschließlich auf Rentabilitätsüberlegungen basierende VeräuBerung von bebauten Grundstücken nicht lohnen: er darf die beim Verkauf freigewordenen stillen Reserven nicht steuerfrei einstreichen und auch nicht im Rahmen der steuerlichen AfA steuermindernd geltend machen, sondern muB sie sich in dem für ihn günstigsten Fall auf die bilanziellen Buchwerte der ersatzweise angeschafften objekte anrechnen lassen. \$6b EStG stellt den gewerblichen Wohnungsinvestor, der im Laufe der Zeit Umschichtungen innerhalb seines Wohnflächenbestandes vorzunehmen plant, rentabilitätsmäßig genauso, als hätte er die verkauften Grundstücke bis zu deren wirtschaftlichem Lebensende genutzt. Es ist offensichtlich, daß sich durch die

110) Dagegen ist es generell nicht möglich, durch Ausnutzung der 4-JahresFrist einen $\mathrm{zinsvortell}$ infolge einer Steuerstundung $\mathrm{zu}$ realisieren. Denn der steuerliche Gewinn des Wohnungsinvestors ist in dem Wirtschaftsjahr, in dem die Riicklage aufzulösen ist, firr jedes volle Wirtschaftsjahr, in dem diese Rilcklage bestanden hat, um 6 Prozent des aufzulösenden Ruicklagenbetrages zu ertöhen;- vgl. Artikel 26, $\mathrm{Nr} .2 \mathrm{~b}$ des Zweiten Haushaltsstrukturgesetzes vam 6.12.1981. 
Anwendung der Vorschriften des $\$ 6 \mathrm{~b}$ EStG die in Abschnitt III.5.2.2.2.3. beschriebenen "Abschreibungsketten" und die damit einhergehenden Effizienzverluste vermeiden lassen; der gewerbliche Wohnungsinvestor erfährt damit eine einkommensteuerliche Behandlung, die insoweit der des in Abschnitt III.5.2.2.2.2. dargestellten Investors entspricht.

Ein weiterer wesentlicher Unterschied zwischen der steuerlichen Behandlung eines nicht-gewerblichen Grundstückseigentümers und der eines gewerbjichen Produzenten von Wohnleistungen besteht darin, daß letzterer mit seinen Mieteinkünften zusätzlich der Gewerbeertragsbesteuerung unterliegt. 111)

Anknüpfungspunkt der Gewerbeertragsteuer ist der Gewerbeertrag, dessen Ermittlung auf dem Gewinn aus Gewerbebetrieb nach \$15, Absatz 1, Ziffer 1 EStG aufbaut (sofern es sich bei dem Wohnungsunternehmen, wie in unserer Analyse unterstellt, um ein einzelkaufmännisches Unternehmen handelt).

Da die Gewerbeertragsteuer, wie auch die Grundsteuer, als Realsteuer konzipiert ist und deshalb nicht die Person des Unternehmenseigners, sondern der stehende, im Inland betriebene Gewerbebetrieb im Mittelpunkt des Interesses steht, sind in diesem Ausgangsbetrag Auswirkungen von individuellen Dispositionen zu neutralisieren, die den einkommensteuerlichen Gewinn mindern und somit einen falschen Eindruck über die Leistungsfähigkeit des Gewerbebetriebs vermitteln. Um welche Beträge der Gewinn vor Steuer zu vermehren bzw. zu vermindern ist, kann man den $\S \S 8$ und 9 GewStG entnehmen.

111) Gewerbekapitalsteuer mu der gewerbliche wohnungsinvestor dagegen nicht zahlen: um eine Doppelbelastung des gewerblichen Grundbesitzes mit zwei Substanzsteuem (Grundsteuer und Gewerbekapitalsteuer) zu vermeiden, sieht §12, Absatz 3, Ziffer 1 EStG diese Steuerbefreiung vor; vgl. auch Abschnitt III.1. dieser Arbeit. 
Sieht man von den Kürzungsmöglichkeiten des \$9 Zif. 1 GewStG einmal ab - ihnen kann man analytisch durch den Ansatz eines geringeren Gewerbeertragsteuersatzes Rechnung tragen -, so ist die für die Praxis wichtigste und für unsere Analyse einzig bedeutsame Hinzurechnungsvorschrift in $\S 8$, Ziffer 1 GewStG enthalten. Sie ordnet $a n$, daß bestimmte $\mathrm{Zinsen,} \mathrm{die} \mathrm{bei} \mathrm{der}$ Ermittlung des ertragsteuerlichen Gewinns als Betriebsausgaben abgezogen worden sind, für die Ermittlung des steuerpflichtigen Gewerbeertrags wieder hinzugerechnet werden müssen. Dies sind im wesentlichen $\mathrm{zinsen}$ auf Schulden, die im Zusammenhang mit der Gründung, dem Erwerb, der Erweiterung oder der Verbesserung des Betriebs entstanden sind. Diese im fachlichen Sprachgebrauch als Dauerschuldzinsen bezeichneten Verbindlichkeiten haben in unserer Analyse ihr Aquivalent in der Betrag rD. Beachteriswert hierbei ist, das nach neuestem Recht lediglich die Hälfte der Dauerschuldzinsen zum ertragsteuerlichen Gewinn hinzugerechnet werden muB.

Unter Berücksichtigung dieser Hinzurechnungsvorschrift erhält man für den vorläufig maßgebenden Gewerbeertrag VGE

$$
V G E=G+\gamma_{3} r D
$$

G steht hierbei für den einkommensteuerlichen Gewinn, in $\gamma_{3}$ schlägt sich die erwähnte Hinzurechnungsvorschrift nieder. Da wir allgemein an den Auswirkungen der Hinzurechnung von Dauerschuldzinsen auf die Finanz- und Investitionsplanung des gewerblichen Wohnungsinvestors interessiert sind, wollen wir für den Parameter $\gamma_{3}$ den Wertebereich $0 \leq \gamma_{3} \leq 1$ vereinbaren.

Daß VGE nur den vorläufigen Gewerbeertrag darstellt, ist durch die Tatsache bedingt, daß die Gewerbeertragsteuer eine durch den Gewerbebetrieb veranlaßte Ausgabe, damit eine Betriebsausgabe nach $\$ 4$, Absatz 4 EStG und als solche bei der Gewinnermittlung abzugsfähig ist. Hieraus ergibt sich ein Berechnungsproblem: denn zur Bestimmung der Gewerbeertragsteuerzahllast muß diese steuer bereits bei der Ermittlung ihrer eigenen Bemessungsgrundlage berücksichtigt werden; dies ist 
aber so lange nicht möglich, solange die Höhe der zu zahlenden Gewerbeertragsteuer unbekannt ist.

In der Praxis behilft man sich hier in aller Regel mit der sogenannten "9/10-Rechnung" des Abschnitts 22, Absatz 2 EStR,"12) die aber die Gewerbeertragsteuerzahllast nicht korrekt auszuweisen vermag (nur bei einem Hebesatz von 222 prozent führt die "9/10-Methode" zum richtigen Ergebnis).

Um zu dem korrekten Ergebnis zu gelangen, muß man zunächst wissen, daß die Gewerbesteuer, wie auch die Grundsteuer, eine "Gemeindesteuer" ist und die Gemeinden hier wie dort eine gewisse Autonomie in der Festsetzung der steuerlast besitzen: nachdem der GewerbeertragsteuermeBbetrag durch Multiplikation des maßgebenden Gewerbeertrags mit einer durch Gesetz festgelegten steuermeßzahl (Sz) bestimmt wurde, erhält man durch die Multiplikation des steuermeBbetrags mit dem vom Gemeindeparlament $\mathrm{zu}$ bestimmenden Hebesatz (H) die $\mathrm{zu} z$ ahlende Gewerbeertragsteuer ( $\left.\mathrm{T}_{\mathrm{gew}}\right)$. Den maßgebenden Gewerbeertrag selbst erhält man, wenn man von dem vorläufig maßgebenden Gewerbeertrag die zu zahlende Gewerbertragsteuer subtrahiert. Folglich läßt sich die Berechnung der Gewerbeertragsteuerzahllast formal wie folgt beschreiben:

(III. 179) $\quad \mathrm{T}_{\text {gew }}=\frac{\mathrm{SZ}}{100} \frac{\mathrm{H}}{100}\left(\mathrm{VGE}-\mathrm{T}_{\text {gew }}\right)$.

Nach einigen einfachen Umformungen erhält man hieraus (III. 180) $\quad \mathrm{T}_{\text {gew }}=\frac{\mathrm{SZ} \cdot \mathrm{H}}{10000+\mathrm{SZ} \cdot \mathrm{H}} \mathrm{VGE}$.

112) ROSE(1978), S.206 ff. 
Anstatt also die "9/10-Methode" anzuwenden und ihre Ungenauigkeiten in Kauf $\mathrm{zu}$ nehmen, bietet sich als Alternative die Multiplikation des vorläufig maßgebenden Gewerbeertrags mit dem effektiven Gewerbeertragsteuersatz $\tau_{\text {gew }}^{*}=\mathrm{SZ} \cdot \mathrm{H} /(10000+\mathrm{SZ} \cdot \mathrm{H})$ an, der geringer ist als das Produkt aus steuermeßzahl und Hebesatz. Wir wollen dieses zweite, korrekte Verfahren auch im Rahmen unserer Analyse anwenden. Der gewerbeertragsteuermindernden Kürzungsvorschrift des $\$ 9$, Ziffer 1 GewStG tragen wir analytisch durch die Anwendung eines effektiven Gewerbeertragsteuersatzes $\tau_{\text {gew }}<\tau_{\text {gew }}^{*}$ Rechnung.

Unter Berücksichtigung der vorangegangenen tberlegungen kann ein gewerblicher Wohnungsinvestor, der neben Gewerbeertragsteuer auch noch Einkommensteuer zahlen muB, folglich in jeder Periode einen Betrag in Höhe von

(III. 131)

$$
\begin{aligned}
A= & z\left(m H-E-r D-T_{e}-T_{g e w}\right)-F E-P_{B} F^{d}+S \\
& +\tau_{e}\left[\gamma_{2} \rho_{1}+\left(1-\gamma_{2}\right) \rho_{2}\right]\left(F_{E}^{d}-P_{B} F^{d}\right)
\end{aligned}
$$

D-Mark entnehmen. 113 )

Für die anstehenden steuerverbindlichkeiten gilt

$$
\left.T_{e}=\tau_{e}\left(m H-E-r D-\mu K^{*}-T_{g e w}\right) ! 14\right)
$$

bzw.

(III. 183)

$$
\mathrm{T}_{\text {gew }}=\tau_{\text {gew }}\left[\mathrm{mH}-\mathrm{E}-\left(1-\gamma_{3}\right) \mathrm{rD}-\mu \mathrm{K}^{*}\right] .
$$

113 ) in (III.181) wird wiederum dem Unstand Rechnung getragen, daß auch der gewerbliche Wohnungsinvestor Wohnungseigentum auf drei unter Kostengesichtspunkten unterschiedlichen Wegen erwerben kann.

114) In den Definitionsgleichungen (III.182) und (III.183) wird unterstellt, daß der Wohnungsinvestor bei der Vornahne von umschichtungen in seinem Wohnungsbestand die erfolgsneutralen Ubertragungsmöglichkeiten des §6b EStG zu nutzen und auf diesem Wege die Besteuerung realisierter hertzuwächse $\mathrm{zu}$ umgehen weiß. 
Substituiert man die Definitionsgleichungen (III.182) und (III.183) in Gleichung (III.181), so läßt sich die zu einem bestimmten zeitpunkt getätigte Entnahme auch schreiben als

(III.184)

$$
\begin{aligned}
A= & z\left[\Theta_{e} e_{\text {gew }}(m H-E-r D)+\tau e^{\mu K^{*}+\tau}{ }_{g e w} \Theta^{\left.\left(\mu K^{*}-Y_{3} r D\right)\right]}\right. \\
& +\tau_{e}\left[\gamma_{2} \rho_{1}+\left(1-\gamma_{2}\right) \rho_{2}\right]\left(F_{\varepsilon}^{d}+P_{B} F^{d}\right)+S-F_{\varepsilon}^{d}-P_{B} F^{d},
\end{aligned}
$$

mit $\Theta_{\mathrm{e}}=1-\tau_{\mathrm{e}}$ und $\Theta_{\text {gew }}=1-\tau_{\text {gew }}$. Der erste Term innerhalb der eckigen Klammer von (III.184) spiegelt die Doppelbelastung bestimmter Bestandteile des cash flow des gewerblichen Wohnungsinvestors wider. Das effektive AusmaB der Doppelbelastung sinkt offensichtlich infolge der Absetzbarkeit der Gewerbesteuer als Betriebsausgaben. Der dritte Term des Klammerausdrucks informiert uns darüber, daß

- der Einsatz von Bauleistungen bei der Produktion von Wohnflächeneinheiten auch im Rahmen der Gewerbeertragsbesteuerung über den Ansatz beschleunigter steuerlicher AfA subventioniert wird - denn Grundlage der Ermittlung des maßgebenden Gewerbeertrags ist der einkommensteuerliche Gewinn; allerdings wird diese Subvention infolge des Betriebsausgabencharakters der Gewerbesteuer ihrerseits im Rahmen der Einkommensbesteuerung erfaßt und sinkt infolgedessen nach Maßgabe des Einkommensteuersatzes.

- (lediglich) der Anteil $\gamma_{3}$ der Dauerschuldzinsen mit Gewerbesteuer belastet wird, die effektive Belastung aber dadurch abnimmt, daß die auf diese Dauerschuldzinsen gezahlte Gewerbesteuer im Rahmen der Einkommensbesteuerung steuermindernd geltend gemacht werden kann.

Mit Hilfe von (III.184) läßt sich nun auch untersuchen, ob und - wenn ja - wie der gewerbliche Wohnungsinvestor seine ursprünglichen, paretooptimalen Finanzierungs- und Investitionspläne unter dem Einfluß von Einkommen- und Gewerbesteuer revidiert. Wir wollen ihm hierbei wiederum unterstellen, daß er durch eine optimale wahl der zeitpfade für die Nettoneuverschul- 
dung, den Erhaltungsaufwand und die Neubauinvestitionen den Barwert der in (III.184) beschriebenen Entnahmen - den Marktwert des Wohnungsunternehmens - zu maximieren beabsichtigt. Folglich lautet das Planungsproblem des gewerblichen wohnungsinvestors

( III.185)

$$
\left\{S, E, E, F^{d}\right\} M_{W}^{M}=\int_{t_{0}}^{\infty} A(t) \exp \left[-z \Theta e^{r(t-t *)}\right] d t
$$

unter den Bedingungen (II.2), (II.3), (II.9)', (II.10), (II.14), (II.16), (II.17a) und (III.184).

Die in laufenden Werten formulierte Hamiltonfunktion für dieses Problem lautet

$$
\begin{aligned}
& H=z\left\{\Theta_{e} \theta_{\text {gew }}(m H-E-r D)+\tau_{e} \mu K^{*}+\tau_{\text {gew }}{ }^{\Theta} e^{\left(\mu K^{*}-Y_{3} r D\right)}\right. \\
& \left.+\tau_{e}\left[\gamma_{2} \rho_{1}+\left(1-\gamma_{2}\right) \rho_{2}\left(F^{d} \varepsilon+P_{B} F^{d}\right)\right]\right\} \\
& -F^{d} \varepsilon-P_{B} F^{d}+S \\
& +P_{H}\left[F^{d} \varphi(\varepsilon)-z(\alpha \delta H-E)\right] \\
& +\lambda_{D} s \\
& +\lambda_{K^{*}}\left(F^{d} \varepsilon-\mu K^{*}\right) \text {. }
\end{aligned}
$$

Aufschlüsse darüber, ob der Wohnungsinvestor unter dem EinfluB der hier betrachteten steuern andere Finanzierungspräferenzen hegt als in der steuerfreien welt, erhält man, wenn man (III.186) partiell nach der Kontrollvariablen S differenziert. Man gewinnt auf diesem Wege die Gleichgewichtsbedingung

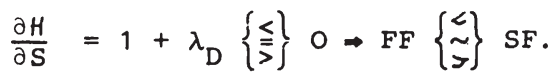


Wenn man darüberhinaus noch weiB, daß für den Schattenpreis $\lambda_{1}$ des Fremdkapitalbestandes gilt

$$
\lambda_{D} \equiv \frac{\partial M_{W}}{\partial D}=-\left(\theta_{\text {gew }}+\gamma_{3} \tau_{\text {gew }}\right)\left\{\begin{array}{l}
= \\
>
\end{array}\right\}-1 \Leftrightarrow \gamma_{3}\left\{\begin{array}{l}
= \\
<
\end{array}\right\} 1,
$$

so kommt man zu dem Ergebnis, daß der gewerbliche wohnunasinvestor unter dem Einfluß der Gewerbeertragsteuer dann eine eindeutige Präferenz für die Finanzierung mit Fremdkapital entwickelt, wenn die Dauerschuldzinsen nicht in voller Höhe zur Ermittlung des maßgebenden Gewerbeertrags hinzugerechnet werden müssen.

Die sich hinter dieser steuerlichen Veränderung der LaissezFaire-Finanzierungspräferenzen verbergenden Zusammenhänge lassen sich mit Hilfe einfacher Arbitrageüberlegungen erhellen: ein gewerblicher Wohnungsinvestor, der vor der Wahl steht, seine Investition entweder mit Krediten zu finanzieren oder die erforderlichen Mittel bei den Unternehmenseignern einzuholen, muß sich darüber im klaren sein, daB der Einsatz von Eigenkapital Opportunitätskosten in Form entgangener Kapitalmarkterträge verursacht; da Kapitalmarkterträge in aller Regel nicht der Gewerbesteuer unterliegen, belaufen sich diese Kosten für jede eingesetzte D-Mark Eigenkapital auf $\theta e^{r}$ D-Mark. Wird stattdessen mit Fremdkapital finanziert, sinken diese Kosten: die Dauerschuldzinsen können sowohl bei der Einkommensteuer als auch (zumindest teilweise) bei der Gewerbeertragsteuer von der jeweiligen Bemessungsgrundlage abgezogen werden.

Nur für den Fall, daß die gesamten Dauerschuldzinsen bei der Ermittlung des steuerpflichtigen Gewerbeertrags hinzugerechnet werden müssen - $\gamma_{3}$ wäre in diesem Fall gleich Eins - käme dieser komparative Kostenvorteil der Fremdfinanzierung nicht zum Tragen.

Der gewerbesteuerinduzierte wandel in den Finanzierungspräfe.renzen gewerblicher Wohnungsinvestoren wird auch Einfluß haben auf deren Investitionsplanung. Wie groß dieser Einfluß ist, hängt entscheidenḍ von der Antwort auf die 
Frage nach der Kreditwürdigkeit des gewerblichen Vermieters $a b$ - denn seine Kreditnachfrage wird unter den genannten Voraussetzungen unbegrenzt sein: nicht nur, daB er seine gesamten Ausgaben für werterhaltende Arbeiten (E) und Neubauvorhaben $\left(F^{d} \varepsilon+P_{B} F^{d}\right)$ am liebsten mit Fremdkapital finanzieren möchte - unter Rentabilitätsgesichtspunkten betrachtet wäre es für ihn rational, darüberhinaus am Kapitalmarkt soviele Fremdmittel wie nur möglich aufunehmen, um damit Entnahmen zu finanzieren. Die Erträge, die die Unternehmenseigner durch eine Anlage der mit Fremdkapital finanzierten Entnahmen am Kapitalmarkt erzielen könnten, wären stets größer als die effektiven Kosten, die dem Gewerbebetrieb infolge der Bedienung dieser zusätzlichen Schulden entstehen würden.

Allerdings werden die kreditgeber nicht bereit sein, dem gewerblichen Wohnungsinvestor einen solch unbeschränkten Kreditspielraum zu gewähren; ihr Engagement wird zumindest langfristig auf den Wert des Betriebsvermögens - in unserer Analyse entspricht dieses dem Betrag $\mathrm{P}_{\mathrm{H}} \mathrm{H}$ - beschränkt sein. Voraussetzung dafür, daß diese Grenze nicht überschritten wird, ist, daß in jeder Periode nicht mehr als der wert der Nettoinvestitionen $\left(\mathrm{P}_{\mathrm{H}} \dot{\mathrm{H}}\right)$ zuzüglich der eingetretenen wertsteigerungen des Grundstückvermögens $\left(\dot{\mathrm{P}}_{\mathrm{H}} \mathrm{H}\right)$ beliehen wird.

Unter Berücksichtigung dieser Schranke erhalten wir für den Gewerbebetrieb in jeder Periode jenseits von $t *$ eine Nettoneuverschuldung in Höhe von

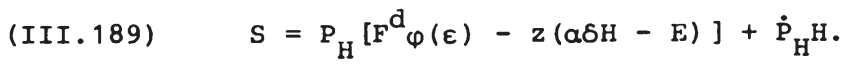

D-Mark.

Zur Bestimmung des Einflusses der steuerinduzierten Veränderung im Finanzierungsverhaj.ten auf die Investitionsplanung des gewerblichen Vermjeters müssen wir wiederum von den in Kapital II formulierten allgemeinen optimalitätsbedingungen (II.23), (II.28), (II.29) und (II.30) ausgehen. 
Unter Berücksichtigung der partiellen Ableitungen von (III. 189)

$$
\begin{array}{ll}
\text { (III. 190) } & \frac{\partial S}{\partial E}=P_{H}, \\
\text { (III. 191) } & \frac{\partial S}{\partial \varepsilon}=P_{H^{\varphi}} \varphi^{\prime}(\varepsilon) F^{d}, \\
\text { (III. 192) } & \frac{\partial S}{\partial F}=P_{H^{\prime}} \varphi(\varepsilon), \\
\text { (III. 193) } & \frac{\partial S}{\partial H}=-P_{H} \alpha \delta+\dot{P}_{H^{\prime}}
\end{array}
$$

sowie der in (III.187) und (III.188) gefundenen Aussage

(III. 194) $\quad \frac{\partial H}{\partial S}=\tau_{\text {gew }}\left(1-Y_{3}\right)$

erhalten wir aus (II.23), (II.28), (II.29) und (II.30) die das gleichgewichtige Investitionsprogramm $k \in n n z e i c h n e n d e n ~ n o t w e n d i-$ gen Bedingungen:

$$
\begin{aligned}
& \text { (III. 195) } \quad \mathrm{P}_{\mathrm{H}}\left\{\begin{array}{l}
<\} \\
\geq
\end{array}\right\} \frac{\theta_{\mathrm{e}}^{\theta_{\text {gew }}}}{1+\tau_{\text {gew }}\left(1-\gamma_{3}\right)} \Rightarrow \mathrm{E}\left\{\begin{array}{l}
=0 \\
=a \delta \mathrm{H}
\end{array}\right. \\
& \text { (III. 196) } \quad \mathrm{P}_{\mathrm{H}} \varphi^{\prime}(\varepsilon)=\frac{1-\tau_{\mathrm{e}}\left[\gamma_{2} \rho_{1}+\left(1-\gamma_{2}\right) \rho_{2}\right]-\lambda_{K^{*}}}{1+\tau_{\text {gew }}\left(1-\gamma_{3}\right)}
\end{aligned}
$$

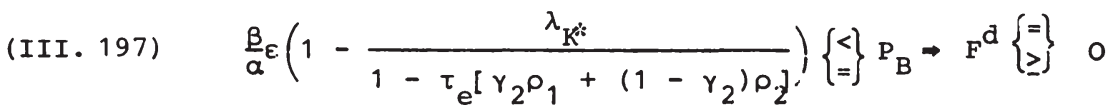

$$
\begin{aligned}
& \text { (III. 198a) } \hat{\mathrm{P}}_{\mathrm{H}}=0 \quad \text { für } t_{\mathrm{o}} \mathrm{t}<\mathrm{t}^{*} \text {, } \\
& \text { (III. 198b) } \quad \hat{\mathrm{P}}_{\mathrm{H}}=-\frac{1}{1+\tau_{\text {gew }}\left(1-\gamma_{3}\right)}\left[\frac{\Theta_{\mathrm{e}}^{\theta_{g e w^{m}}}}{\mathrm{P}_{\mathrm{H}}}-\Theta_{\mathrm{e}} \mathrm{r}\right]+\alpha \delta \text { für } t \geq \mathrm{t}^{*} \text {. }
\end{aligned}
$$


Wie aus Bedingung (III.195) hervorgeht, ändert sich unter dem Regime der Gewerbeertragsteuer das paretooptimale Werterhaltungsprogramm der Laisser-Faire-ökonomie nicht: da wir in unserer Analyse $\mathrm{P}_{\mathrm{H}}>1$ unterstellt haben und sich die Kosten werterhaltender Arbeiten unter dem Einfluß der Einkommens- und Gewerbeertragsbesteuerung verringern - Erhaltungsaufwand kann sowohl im Rahmen der Einkommensteuer als auch im Rahmen der Gewerbesteuer sofort abgeschrieben werden, zudem sinken unter dem Einfluß der Gewerbeertragsteuer die effektiven Finanzierungskosten werterhaltender Arbeiten -, wird der abnutzungsbedingte Substanzverzehr an Gebäudekapital in jeder Periode durch Renovierungsarbeiten ausgeglichen.

Mit Hilfe der Bedingungen (III.196) bis (III.198) läBt sich beschreiben, wie sich die zeitliche Entwicklung der Wohnungsund Baulandmärkte verändert, wenn zusätzlich zu einer Steuer auf "Einkünfte aus Gewerbebetrieb" ein€ Steuer auf den nach geltendem Recht ermittelten Gewerbeertrag erhoben wird. Wir müssen uns hierzu nur in Erinnerung rufen, daß sich anhand der Bedingungen (III.196) und (III.198b) Rückschlüsse auf die Lage und auf eine mïgliche Verschiebung der Angebotskurve bzw. der Nachfragekurve ziehen lassen:

Ein Blick auf Bedingung (III.196) zeigt uns, daß sich die Angebotskurve $A^{\prime}$ in $A b b .15$ unter dem EinfluB der Gewerbeertragsteuer nach rechts verschiebt: wurde der Einsatz von Bauleistungen schon beim nicht-gewerblichen (und nicht-veräußernden) Wohnungsinvestor im Zuge des Eigentumserwerbs und des Ansatzes steuerlicher AfA im Vergleich zur Laisser-Faire-Situation begünstigt, so wird der damit einhergehende expansive Effekt durch die Erhebung einer Gewerbeetragsteuer noch zusätzlich dadurch verstärkt, daß

- der gewerbliche Wohrungsinvestor durch ein Ausweichen auf die Kreditfinanzierung die Vermögensposition der Unternehmenseigner verbessern kann,

- der gewerbliche Wohnungsinvestor im Vergleich zu seinem nichtgewerblichen Konkurrenten in den Genuß zusätzlicher staatlicher Subventionen gelangt, da der Faktor "Bauleistungen" 
auch im Rahmen der Gewerbeertragsbesteuerung über den Ansatz steuermindernder Absetzungen für Abnutzung begünstigt wird.

Den formalen Beleg für die Richtigkeit dieser Uberlegungen erhalten wir, wenn wir in die allgemeine Form der Angebotsfunktion aus (II.79) die Inverse der Gleichgewichtsbedingung (III.197) einsetzen; man errechnet dann für die Angebotskurve $\mathrm{A}^{\prime \prime}$ in $\mathrm{Abb} .17$ den funktionalen zusammenhang

$$
H(t *)=\Phi\left\{\varphi\left[\varphi^{\prime-1}\left(\frac{1-\tau_{e}\left[\gamma_{2} \rho_{1}+\left(1-\gamma_{2}\right) \rho_{2}\right]-\lambda_{K^{*}}}{\left[1+\tau_{\text {gew }}\left(1-\gamma_{3}\right)\right] P_{H}}\right)\right]\right\} .
$$

Der Quotient in der runden Klammer von (III.199) nimmt unter dem EinfluB der Gewerbeertragsteuer $a b$ :

- der Nenner dieses Bruchs, in ihm schlägt sich der komparative Kostenvorteil der Kreditfinanzierung nieder, steigt wegen $\gamma_{3}<1$ nach Maßgabe des (effektiven) Gewerbeertragsteuersatzes;

- der zähler verliert gewerbeertragsteuerbedingt an Wert, da der Schattenpreis $\lambda_{K} *$ des bilanziellen Kapitalstocks infolge der Ansetzbarkeit einkommensteuerlicher AfA im Rahmen der Gewerbeertragsbesteuerung mit dem steuersatz $\tau_{\text {gew }}$ steigt; für $\lambda_{K}$ * errechnen wir

(III.200)

$$
\lambda_{K *} \equiv \frac{\partial M_{W}}{\partial K^{*}}=\frac{\mu\left(\tau_{e}+\tau_{g e w} \theta_{e}\right)}{\theta_{e} r+\mu} .
$$

Da annahmegemäß die Ableitungseigenschaften $\Phi^{\prime}, \varphi^{\prime},-\varphi^{\prime}{ }^{\prime}<0$ Gültigkeit besitzen, muß die Rechtsverschiebung der Angebotskurve im Falle des gewerblichen Wohnungsinvestors stärker ausfallen, verglichen mit dem Fall, in dem die Finanzbehörden die Vermietung von Wohnungseigentum als Vermögenverwaltung interpretieren und der Wohnungsinvestor einen späteren Verkauf seiner Wohnimmobilie nicht ins Auge faßt. 
Daß nicht nur die Ausgaben für Bauleistungen, sondern auch die für Bauland von der steuerbedingten Verringerung der Finanzierungskosten profitieren, geht aus Bedingung (III. 197) hervor: die Hinzurechnungsvorschrift des §8, Ziffer 1 GewStG sie findet formal in dem Parameter $\gamma_{3}$ Ausdruck - übt keinen Einfluß aus auf die wahl der gleichgewichtigen Kapitalintensität der Bebauung. Trotzdem wird sich die schon als Folge der gegenwärtig praktizierten Einkommensbesteuerung beobachtbare Neigung der Wohnungsinvestoren $z u$ einer kapitalintensiveren Bebauungsweise beim gewerblichen Investor noch verstärken - wie in (III. 200) gezeigt wurde, wächst äer Schattenpreis des bilanziellen Kapitalstocks $\lambda_{\mathrm{K}^{*}}$, der in die Gleichgewichtsbedingung (III. 197) Eingang findet, mit dem Gewerbeertragsteuersatz.

Ebenso wie die Angebotskurve verändert auch die Nachfragekurve ihre Position unter dem Einfluß der Gewerbeertragsteuer. Bemerkenswert hierbei ist jedoch, daß sich nicht sagen läßt, in welche Richtung diese Verschiebung erfolgt: löst man Bedingung (III.198b) nach dem Häuserpreis $P_{H}$ auf, so erhält man als Ergebnis die Gleichung für die Nachfragekurve N'' in Abb.17 :

(III. 201)

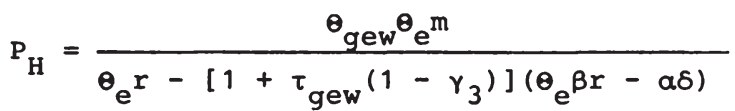

Wie man leicht erkennt, nimmt zwar der zähler des Quotienten in (III. 201) infolge der Doppelbelastung der Mieteinkünfte mit Einkommen- und Gewerbeertragsteuer im Vergleich sowohl zur Laisser-Faire-Welt als auch zum Fall des Wohnungsinvestors aus Abschnitt III.5.2.2.2.1. ab; ebenso eindeutig ist aber auch die gewerbesteuerbedingte Abnahme des Nenners dieses Quotienten: dem rentabilitätsmindernden Effekt der Doppelbelastung wirkt der rentabilitätssteigernde Effekt der Entscheldung für die Kreditfinanzierung entgegen. Ob die Attraktıvität einer Vermögensanlage in Form von Wohnungseigentum gewerbesteuerbedingt geringer 
wird oder zunimmt, läßt sich deshalb ohne Kenntnis der entsprechenden Parameterwerte nicht sagen.

Dieser Mangel an Eindeutigkeit hat auch Konsequenzen für die Beschreibung der Gewerbesteuerinzidenz. Insbesondere läßt sich vor dem Hintergrund der Analyseergebnisse nicht sagen, ob der gewerbliche wohnungsinvestor - wie üblicherweise unterstellt wird - infolge der zusätzlichen Belastung seiner Einkünfte aus Gewerbebetrieb mit Gewerbeertragsteuer gegenüber seinem nicht-gewerblichen Konkurrenten einen Rentabilitätsnachteil erleidet.

In Abb.17 wird eine Situation beschrieben, in der genau das Gegenteil der Fall ist. Die Kurven $A^{\prime}$ und $N^{\prime}$ beschreiben die Angebots- bzw. Nachfrageverhältnisse am Markt für Wohnleistungseinheiten, die sich im Anschluß an die Besteuerung der Mieteinkunfte des nicht-gewerblichen Wohnungsinvestors gemä $\beta$ \$21 EStG eingestellt haben (vgl. Abb.15). Zwar erhöhen sich infolge der zusätzlichen Belastung der Mieteinkünfte im speziellen Fall der Abb.17 die Nutzungskosten des im gewerblichen Wohnungssektor gebundenen Vermögens; der damit einhergehende, die Vermögensbildung in Form von Wohnimmobilien diskriminierende Effekt reicht aber nicht aus, die Kostenvorteile zu kompensieren, die die Finanzierung der Investitionsausgaben mit Fremdkapital und die zusätzlichen Abschreibungsvergünstigungen zu bieten vermögen. In diesem speziellen Fall kann man als unmittelbare Reaktion auf die Einführung einer Gewerbeertragsteuer einen kurzfristigen Neubauboom im Sektor der gewerblichen Wohnungswirtschaft beobachten. Die damit einhergehende Angebotserhöhung an Wohnleistungseinheiten in $t_{0} \mathrm{mu} B$ bei gegebener Nachfrage nach solchen Leistungen $\mathrm{zu}$ einem Absinken des Mietenniveaus und des Häuserpreises in $t_{0}$ führen; dieses Absinken wird zudem dauerhaft sein, da aufgrund der von der steuer unbeeinfluBten Wachstumsrate $\hat{H}$ der Wohnflächenbestand $z u$ jedem zeitpunkt jenseits von $t_{0}$ grösser ist, als er es in einer Welt ohne Gewerbeertragsteuer gewesen wäre; dauerhaft angehoben wird der Zeitpfad für 
Abb. 17

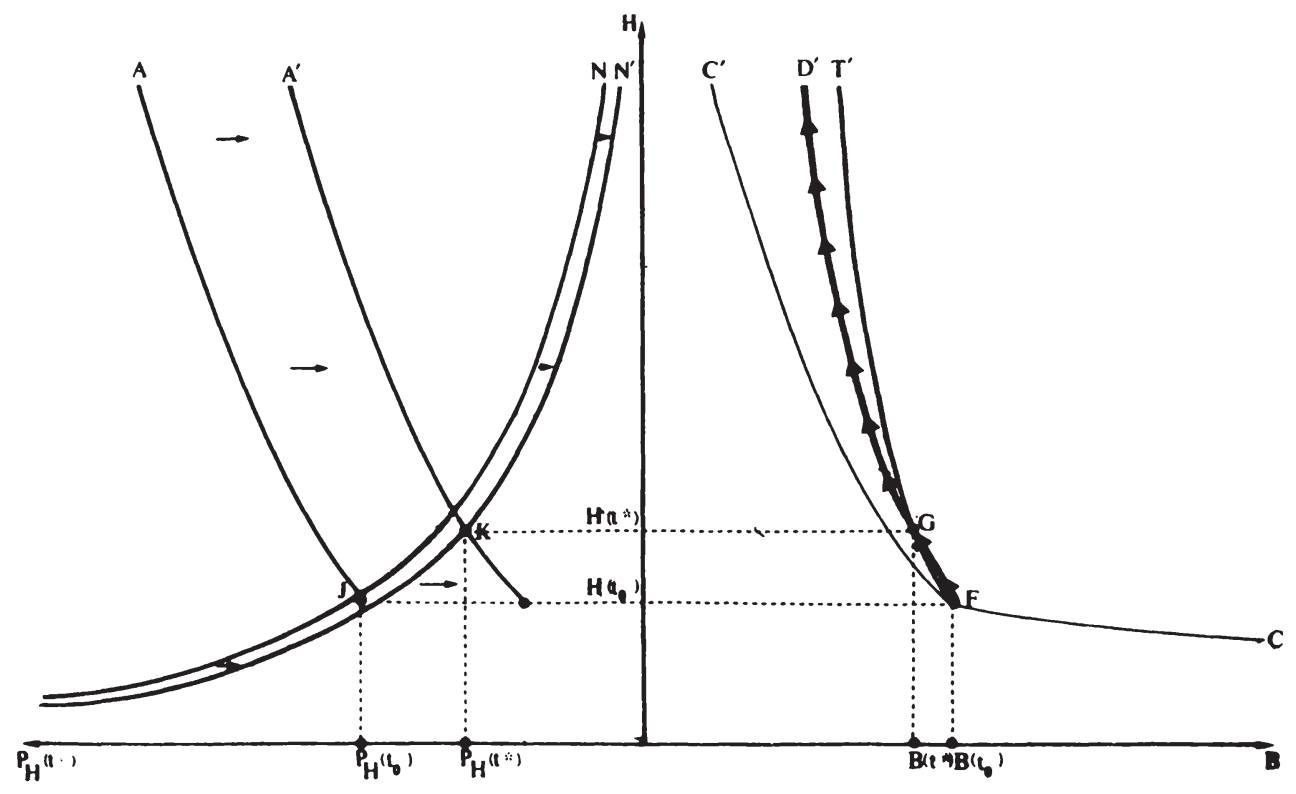

- den Bodenpreis - die Steuervergünstigungen vergrößern die Zahlungsberejtschaft des Wohnungsinvestors für baureife Grundstücke - und

- die Kapitalintensität der Bebauung.

Da sich auch der Wert der Schrumpfungsrate $\hat{B}$ des Baulandbestandes unter dem EinfluB der Gewerbeertragsbesteuerung nicht ändert, sinkt der Zeitpfad des Baulandbestandes steuerbedingt dauerhaft $a b$. 
5.3. Perspektiven einer effizente(re) r. Form der Ertragsbesteuerung des wohnungssektors

Wie die vorangegangenen Abschnitte gezeigt haben, wird das geltende Einkommensteuerrecht der Forderung nach einer effizienten Form der Besteuerung des Wohnungssektors in keiner Weise gerecht: - der Wohnungsinvestor kann zunächst auf mehreren, aufgrund einkommensteuerlicher Unzulänglichkeiten unterschiedlich teuren Wegen Wohnungseigentum erwerben:

o er kann seine Wohnung(en) von einem Bauherrn erwerben, der realisierte Veräußerungsgewinne nicht versteuern muß;

o er kann seine Wohnung(en) von einem Bauherrn erwerben, der zur Versteuerung aufgedeckter stiller Reserven verpflichtet ist;

o er kann seine Wohnung(en) in der Funktion des Bauherrn selbst errichten.

Wenn man darüberhinaus noch bedenkt, daß der (nicht-gewerbliche) Bauherr die Möglichkeit hat, die steuerrelevanten Gestehungskosten der Wohnimmobilie(n) durch das Einbringen nichtpekuniarisierter Eigenleistungen zu senken, kann man alleine im Bereich des freifinanzierten Wohnungsbaus mindestens fünf unterschiedlich rentable Erwerbsformen unterscheiden.

- Unabhängig von der Erwerbsform wird auch innerhalb der Nutzungsphase des Grundstücks massiv gegen den steuerlichen Gleichbehandlungsgrundsatz verstoßen: je nachdem, ob der Wohnungsinvestor sein Wohnungseigentum als Nichtgewerbetreibender

- selbst nutzt oder

o zumindest teilweise vermietet oder

o im Falle der Vermietung vor dessen ökonomischem Lebensende veräuBert oder

o im Falle der Teilvermietung nach einer gewissen zeit vollständig selbst nutzt,

bzw. als Gewerbetreibender vermietet, nimmt die Last der Einkommensteuer für ihn sehr unterschiedliche Ausmaße an.

Faßt man Erwerbs- und Nutzungsphasen zusammen, so lassen sich alleine im Bereich des freifinanzierten Wohnungsbaus min- 
destens 25 unterschiedlich rentable Möglichkeiten aufzeigen, wie man Wohnimmobilienvermögen bilden und nutzen kann. Und sieht man von der Pauschalierung des Nutzungswertes einmal ab nur in diesem szenario stellt sich der (mit Eigenkapital finanzierende) Wohnungsinvestor unter Rentabilitätsgesichtspunkten besser als in der steuerfreien Welt -, so läßt sich bei keiner dieser Möglichkeiten sagen, ob die Wohneigentumsbildung durch die geltenden einkommen- und gewerbesteuerlichen Regelungen im Vergleich zu den anderen in unserem Modell betrachteten Vermögensformen begünstigt oder benachteiligt wird.

E's darf deshalb auch nicht überraschen, wenn Steuerpflichtige, die vor der Frage stehen, ob sie sich für ein Mehrfamilienhaus, ein Zweifamilienhaus, ein Einfamilienhaus oder eine Eigentumswohnung entscheiden sollen, im Regelfall überfordert sind, die damit einhergehenden steuerlichen Konsequenzen im einzelnen abzusehen; denn dazu sind die gesetzlichen Bestimmungen über die verschiedenen Arten der Nutzungswert- bzw. Gewinnermittlung mit den unterschiedlichen Möglichkeiten, Schuldzinsen, Abschreibungen und andere Werbungskosten bzw. Betriebsausgaben steuermindernd geltend $z u$ machen, für den juristisch Unbewanderten zu schwer verständlich und für den in Finanzmathematik Ungeübten in ihren ökonomischen Folgen kaum abzuschätzen. Man darf deshalb davon ausgehen, daß bei der Investitionswahl Entscheidungen getroffen werden, die in wirklicher Kenntnis der zum Teil erheblichen Rentabilitätsunterschiede zwischen den diversen Eigentumsformen in vielen Fällen anders ausfallen würden und die aus diesem Grunde die oben beschriebenen Wohlfahrtsverluste im Zweifelsfalle noch erhöhen.

Der einzige Ausweg aus dem gegenwärtigen Dilemma kann nur darin bestehen, daß man die Erträge ökonomisch gleichartiger Aktivitäten - hier ist dies die Erstellung und wirtschaftliche Verwertung von Wohnraum - im Rahmen der Ertragsbesteuerung auch einheitlich behandelt. Und wenn man gewerbliche Wohnungsinvestoren nicht ebenfalls von der Einkommensteuerpflicht entbinden 
will, kann dieser Forderung nur die Einführung der "Investitionsgutlösung" gerecht werden.

Streiten kann man sich freilich darüber, in welcher Form diese einkommensteuerliche konzeption - zunächst zumindest eingeführt werden sollte. Es wäre sicherlich nicht sinnvoll, von Anfang an ein Verfahren zu implementieren, das dem in Kapitel III.1. beschriebenen Idealtypus entspricht. Solange nicht auch in allen anderen sektoren unserer Volkswirtschaft wertänderungen des Vermögens zum Zeitpunkt ihrer Entstehung erfaßt werden, müßte ein solcher Alleingang im Bereich des freifinanzierten Wohnungsbaus zwangsläufig zu neuen Wohlfahrtsverlusten führen.

Andererseits ist aber nicht einzusehen, warum ausgerechnet im nicht-gewerblichen Teil des Wohnungssektors Wertsteigerungen des Grundvermögens steuerfrei realisierbar sein sollen. Mit dem Prinzip der Besteuerung nach der Leistungsfähigkeit steht diese Regelung jedenfalls nicht im Einklang. Es wäre ein erster Schritt in die richtige Richtung, wenn eine einheitliche steuerliche Behandlung der Erträge aus Vermietung und Verpachtung verwirklicht werden könnte, bei der das $z u$ versteuernde Einkommen aus Wohnimmobilienvermögen im Rahmen einer Uberschußrechnung ermittelt und die beim Verkauf von Grundstücken aufgedeckten stillen Reserven auch beim nicht-gewerblichen wohnungsinvestor den Bestimmungen des $\S 6 \mathrm{~b}$ EStG gemäß erfaßt werden würden.

In der Vergangenheit setzte sich derjenlge, der solche Vorschläge in die wohnungspolitische Diskussion einzubringen versuchte, fast automatisch dem vorwurf aus, den Blick für das politisch Machbare verloren zu haben. Allerdings vermag der Hinweis auf politische Sachzwänge nicht zu überzeugen, wenn man sieht, daß in anderen marktwirtschaftlich organisierten Volkswirtschaften systeme der Einkommensbesteuerung implementiert wurden und werden, deren fiskalische Konsequenzen für die öffentlichen Hände wesentlich einschneidender sind als die der vorgeschlagenen Investitionsgutlösung mit Abschreibungen pro rata tem- 
poris und einer unbeschränkten Abzugsfähigkeit von Fremdkapitalzinsen. An erster Stelle wären hier die USA zu nennen: das von der Reagan-Administration seit 1981 eingeführte und bis 1987 stufenweise auszubauende Accelerated Cost Recovery System ist ein erster grober Schritt hin auf ein System der Einkommensbesteuerung mit Sofortabschreibung der Investitionsausgaben. Wie Sinn gezeigt hat, drohen, wenn das Programm 1987 voll in Kraft tritt und das derzeit im Rahmen von Doppelbesteuerungsabkommen bezüglich grenzüberschreitender Zinszahlungsströme geltende Wohnlandprinzip nicht zugunsten des Quellenlandprinzips aufgegeben wird, ausgeprägte Verzerrungen der weltweiten Kapitalstruktur. 115) Auch der bundesdeutsche Gesetzgeber wird dann erhebliche Anstrengungen unternehmen müssen, um einer so motivierten Kapitalflucht zu begegnen. Er wird dann sehr wahrscheinlich vor der Aufgabe stehen, auch in der Bundesrepublik Deutschland ein system der Ertragsbesteuerung einzuführen, das eine Sofortabschreibung der Investitionsgüter erlaubt.

Vorschläge, wie eine solche steuer auszusehen habe, sind in der steuertheoretischen Literatur selten anzutreffen; im wesentlichen beschränken sie sich auf die Beiträge des MEADE-Committees (1978) und von SINN (1982).116)

Der vom MEADE-Committee favorisierte Vorschlag sieht vor, den Zahlungsüberschuß aus realwirtschaftlichen und finanzwirtschaftlichen Transaktionen des Steuerpflichtigen zu belasten. Im Ergebnis läuft dieser Vorschlag darauf hinaus, die Ausschüttungen des Wohnungsunternehmens

$$
A=z(m H-E-r D)-F_{\varepsilon}^{d}-P_{B} F d+S
$$

der Einkommensbesteuerung $z u$ unterwerfen. Insbesondere muß bei diesem System, um die Finanzierungsneutralität der Einkommensbesteuerung $\mathrm{zu}$ erhalten, eine Besteuerung der zinseinkünfte der Haushalte unterbleiben.

115) $\operatorname{SINN}(1982)$.

116) Vgl. hierzu auch BROWN(1948) und KAY/KING(1978). 
Vor allem in diesem letzten Punkt zeigt sich ein entscheidender Nachteil des vom MEADE-Committee unterbreiteten Vorschlags gegenüber der von SINN zur Diskussion gestellten Konzeption: letztere ist in der Lage, dieselben Allokationsergebnisse zu produzieren wie das MEADsche cash-flow-System, ohne daB hierzu die Abschaffung bestimmter Teile der geltenden Einkommensteuer erforderlich wäre. ${ }^{117)}$ Aus diesem Grunde wollen wir uns im folgenden auf die Analyse jener allokativen Konsequenzen beschränken, die der Vermögensbildung im Wohnungssektor durch die Implementierung des SINNschen Mischsystems erwachsen würden.

Dieses System entspricht in der steuerlichen Behandlung finanzwirtschaftlicher Transaktionen dem SCHANZschen Konzept der Einkommensbesteurung:

- Schuldzinsen sind in voller Höhe abzugsfähig,

- Zinseinkünfte sind in voller Höhe $z u$ versteuern,

- die auf dem Wege der Nettoneuverschuldung zufließenden Geldbeträge bleiben - im Gegensatz zum Vorschlag des MEADE-Committees - einkommensteuerfrei.

In der Behandlung realwirtschaftlicher Transaktionen stimmt es dagegen mit dem cash-flow-System des MEADE-Committees überein; speziell für Wohnungsunternehmen hätte dies zur Folge, daß - die Ausgaben für Bauleistungen $\left(E+F^{d} \varepsilon\right)$ und

- die Ausgaben für Bauland $\left(P_{B} F^{d}\right)$

zum zeitpunkt des Fälligwerdens in voller Höhe von der Einkommensteuerbemessungsgrundlage abgezogen werden dürfen.

Demnach hätte der repräsentative Wohnungsinvestor in jeder Periode eine Einkommensteuerzahllast in Höhe von

(III. 203)

$$
T_{e}=\tau_{e}\left(m H-E-r D-F{ }_{\varepsilon}-P_{B} F^{d}\right)
$$

117) Der vom Meade-Committee unterbreitete Vorschlag hat darüber hinaus einen weiteren Nachteil: da auf der Unternehmensseite eine einkommensteuerliche Zinsverrechnung stattfindet, im Sektor der privaten Haushalte dagegen nicht, bedingt er einen Einkunftsartendualismus. Zu welchen Nachteilen dies führen kann, wurde in den vorangegangenen Abschnitten gezeigt. Beim SINNschen Mischsystem ist dieser Einkunftsartendualismus dagegen ebenso überflüssig wie bei den SCHANZ-HAIG-SIMONS-Systemen. 
D-Mark zu tragen.

Ergänzt man das Laissez-Faire-Optimierungsproblem des Wohnungsinvestors [vgl.(II.19)] um die Einkommensteuerverpflichtungen aus (III. 203) - wobei zusätzlich zu berücksichtigen ist, daß infolge der steuerpflicht der Zinseinkommen bei der Diskontierung zukünftiger Ausschüttungen der Nettorealzins $\theta_{\mathrm{e}} r$ anzuwenden ist -, läßt sich das Entscheidungsproblem des Wohnungsinvestors unter Berücksichtigung der Restriktionen (II.2), (II.3), (II.9)', (II.10), (II.14), (II.16) und (II.17.a) durch die folgende, in laufenden Werten definierte Hamiltonfunktion beschreiben:

$$
\begin{aligned}
H & =\theta_{e}\left[z(m H-E-r D)-F \varepsilon-P_{B} F^{d}+S\right] \\
& +P_{H}\left[F_{\varphi}^{d}(\varepsilon)-z(a \delta H-E)\right] \\
& +\lambda_{D} S .
\end{aligned}
$$

Wie man leicht erkennt, ist der Wohnungsinvestor auch unter dem Regime des Mischsystems indifferent bezüglich der sich ihm eröffnenden Finanzierungswege: wie im scHanzschen system der Einkommensbesteuerung sind Fremdkapitalzinsen in voller Höhe abzugsfähig und werden Erträge alternativer Vermögensformen in vollem Umfang der Einkommensbesteuerung zugeführt. Auch hier ließen sich für die Unternehmenseigner über eine (marginale) Veränderung der Finanzierungsstruktur zugunsten der einen oder der anderen Finanzierungsform keine zusätzlichen Vermögensvorteile erzielen.

Auch am paretooptimalen Werterhaltungsprogramm des Wohnungsunternehmers wird sich unter dem Regime des Mischsystems nichts ändern: da die effektiven Kosten werterhaltender Arbeiten infolge der Sofortabschreibung sinken und der Häuserpreis annahmegemäB den Wert einer D-Mark übertrifft, werden alle abnutzungsbedingten substanzmenderungen des Gebaudekapitalstocks in jeder Periode durch entsprechende Renovierungsarbeiten wieder aufgeholt. 
Die notwendigen Bedingungen für die gewinnmaximalen Neubauund Bestandspläne des repräsentativen Wohnungsinvestors lauten wie folgt:

(III. 205)

$$
\mathrm{P}_{\mathrm{H}^{\prime}} \varphi^{\prime}(\varepsilon)=\Theta_{\mathrm{e}^{\prime}}
$$

(III. 206)

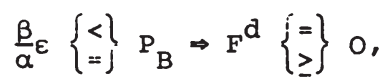

(III. 207a)

$$
\hat{\mathrm{P}}_{\mathrm{H}}=0
$$

für $t_{0} \leq t<t *$,

(III. 207b)

$$
\hat{\mathrm{P}}_{\mathrm{H}}=-\frac{\Theta_{\mathrm{e}}^{\mathrm{m}}}{\mathrm{P}_{\mathrm{H}}}+\Theta_{\mathrm{e}} \mathrm{r}+\alpha \delta \text { für } t \geq t * \text {. }
$$

$D a B$ infolge der Sofortabschreibung der Ausgaben für Bauleistungen die effektiven Kosten dieses Produktionsfaktors sinken, bestätigt die Gleichgewichtsbedingung (III.205) : im neuen Gleichgewicht nach Steuer wird das Wertgrenzprodukt der letzten Einheit dieses Produktionsfaktors geringer sein als in der steuerfreien Welt. Da sich die Kapitalintensität der Bebauung unter dem Einfluß der Einkommensbesteuerung des Wohnungsinvestors nicht verändert - wie aus Bedingung (III.206) hervorgeht, wird der Einsatz des Produktionsfaktors "Bauland" unter dem Regime des Mischsystems im Gegensatz zu den geltenden Verfahren der Einkommensbesteuerung nicht mehr diskriminiert - kann (III.205) nur dann erfüllt sein, wenn im neuen Gleichgewicht ein im Vergleich zur steuerfreien Welt geringerer Häuserpreis gilt. Bei gegebener Nachfrage nach Wohnleistungen ist das Sinken des Häuserpreises in $t_{0}$ gleichbedeutend mit einem Anstieg des Wohnflächenbestandes (des Wohnleistungsangebots): dadurch, daß die öffentliche Hand an den Gesamtkosten des neubebauten Grundstücks unmittelbar partizipiert, wird es für den Wohnungsinvestor attraktiv, seine ursprünglichen, für to geplanten Wohnungsneubauinvestitionen im unmittelbaren AnschluB an die erstmalige Erhebung der 
Steuer auszudehnen.

Für die Höhe der Nutzungskosten des im Wohnungssektor gebundenen Vermögens wird die Einräumung einer Sofortabschreibung ebenfalls Folgen haben

- ein nutzungskostenerhöhender EinfluB ist dem Mischsystem insofern immanent, als die Mieteinnahmen versteuert werden müssen und die abnutzungsbedingten Wertminderungen des Wohnflächenbestandes nicht von der Bemessungsgrundlage abzugsfähig sind;

- als nutzungskostensenkend erweist sich dagegen, daß auch die Erträge alternativer Aktiva besteuert werden und nachfragebedingte Wertsteigerungen des Wohnimmobilienvermögens steuerfrei bleiben.

$D a B$ der die Vermögensbildung in Form von Wohngrundstücken begünstigende Einfluß des Mischsystems dominiert, wird offensichtlich, wenn man in den Bedingungen (III.205) bis (III.207b) für den Häuserpreis $P_{H}$ den modifizierten Häuserpreis $\bar{P}_{H}={ }_{H} / \theta_{e}$ setzt. Man erhält auf diesem Wege die Gleichgewichtsbedingungen

$$
\begin{aligned}
& \text { (III. 205)' } \quad \overline{\mathrm{P}}_{\mathrm{H}^{\prime}} \varphi^{\prime}(\varepsilon)=1, \\
& \left(\text { III. 206)' } \quad \frac{\beta}{\alpha} \varepsilon\left\{\begin{array}{l}
< \\
=
\end{array}\right\} P_{B} \Rightarrow F^{d}\left\{\begin{array}{l}
= \\
\geq
\end{array}\right\} 0\right. \text {, } \\
& \text { (III. 207a)' } \hat{\mathrm{P}}_{\mathrm{H}}=0 \quad \text { für } t_{\mathrm{O}} \leq t<t^{*} \text {, } \\
& \text { (III.207b)' } \quad \hat{\bar{P}}_{H}=-\frac{m}{\bar{P}_{H}}+\theta_{e} r+a \delta \text { für } t \geq t * .
\end{aligned}
$$

Offensichtlich übt das Mischsystem per saldo einen Einfluß lediglich auf die Nutzungskosten des im Wohnungssektor gebundenen Vermögens aus - die Nutzungskosten sinken nach Maßgabe des Einkommensteuersatzes.

Graphisch kommt diese steuerinduzierte Rentabilitätssteigerung in einer Linksverschiebung der Nachfragekurve $\mathrm{N}$ aus Abb.7 zum Ausdruck. Den analytischen Beleg für diese Behauptung erhält man, wenn man die Gleichgewichtsbedingung (III.207b)' nach 
$\overline{\mathrm{P}}_{\mathrm{H}}$ auflöst; man gewinnt auf diese Weise die Funktion der Nachfragekurve $\mathrm{N}^{\prime}$ in $\mathrm{Abb} .18$ :

(III.208)

$$
\bar{P}_{H}=\frac{m(a / H)}{(1-\beta) \Theta e^{r+\alpha \delta}} \text {. }
$$

In (III.208) enthalten sind die Reaktionen des Bodenmaklers auf die Implementierung des Mischsystems: offensichtlich bleiben auch im Rahmen dieser Konzeption die Wertsteigerungen des Baulandbestandes von der Einkommensteuer befreit; die Reaktionen des Bodenmaklers auf die Erhebung einer solchen Steuer werden deshalb dieselben sein, die wir in Abschnitt III.5.2.1.1. dieser Arbeit beschrieben haben: es kommt infolge der steuerbedingt gestiegenen Rentabilität dieses Vermögensbestandes $\mathrm{zu}$ einer verstärkten Hortung von Bauland, in deren Gefolge sowohl der gleichgewichtige Bodenpreis $\left(\hat{P}_{B}=\Theta_{e} r\right)$ als auch der gleichgewichtige Häuserpreis $\left(\hat{\mathrm{P}}_{\mathrm{H}}=\mathrm{B \Theta}_{\mathrm{e}} r\right)$ mit einer geringeren Rate wachsen als in der steuerfreien Welt.

Der Wert des Quotienten in (III.208) nimmt unter dem Einfluß der Einkommensbesteuerung (und unter sonst gleichen Bedingungen) zu: zu jedem Wohnflächenbestand korrespondiert somit ein höherer Häuserpreis $\overline{\mathrm{P}}_{H^{\prime}}$, der Graph der Nachfragefunktion rückt, wie im linken Quadranten der Abb.18 dargestellt, nach links.

offensichtlich ist das Mischsystem zumindest im Rahmen der Partialanalyse nicht allokationsneutral; würde man dieses system in eine steuerfreie Welt einführen bzw. ein investitionsneutrales SCHANZsches system durch dieses system ersetzen, könnte man als unmittelbare Reaktion auf die Reform einen kurzfristigen Boom am Wohnungsneubaumarkt beobachten, in dessen Verlauf der Wohnungsbestand auf das nach steuer optimale Niveau $H\left(t^{*}\right)$ angehoben würde. Parallel dazu würde das Mietenniveau sinken und der Bodenpreis steigen. Der Bestand an baureifen Grundstücken wïrde zunächst abnehmen. 
$\mathrm{Abb} .13$

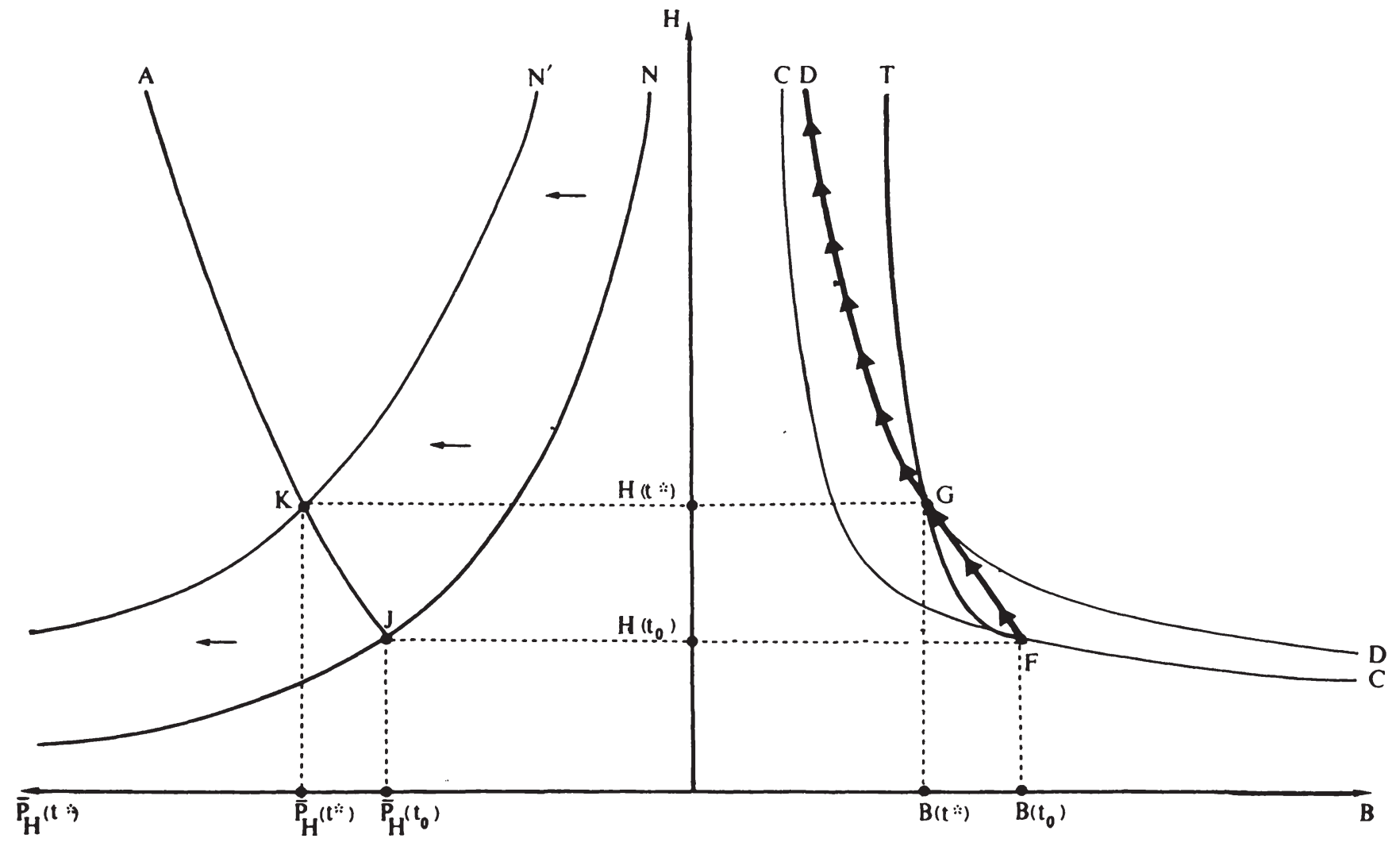


Da die Besteuerung des Wohnungsinvestors keinen Einfluß hat auf die modellendogenen Wachstumsraten, gelten für die langfristige Inzidenz des Mischsystems die in Abschnitt III.5.2.1.1. aufgezeigten Zusammenhänge: da der Wohnflächenbestand infolge der Reaktion des repräsentativen Bodenmaklers auf die Erhebung dieser Steuer mit einer höheren Rate wächst als in der steuerfreien Welt, werden zu jefem. Zeitpunkt jenseits von t* mehr wohnflächeneinheiten angebotkn, als dies unter dem Regime einer investitionsneutralen SCHANZschen Einkommensteuer der Fall gewesen wäre. Entsprechend dauerhaft wird bei gegebenem zeitpfad der wohnleistungsnachfrage das Absinken des Zeitpfades der Wohnungsmieten sein. Der Bodenpreis wird - trotz seines anfänglichen Anstiegs - jenseits eines absehbaren zeitpunktes $t * *$ unterhalb seines Laisser-Faire-Niveaus liegen. Und auch der Baulandbestand wird zu jedem zeitpunkt jenseits von $t *$ kleiner sein als in einer welt, in der eine der ScHANzschen Konzeption folgende Einkommensteuer erhoben wird.

Insofern ist das Mischsystem unter normativen Gesichtspunkten grundsätzlich anders zu beurteilen als die streng am Leistungsfähigkeitsprinzip ausgerichtete SCHANZsche Einkommensteuer. Zwar wird sich - die Implementierung des Mischsystems in allen anderen Sektoren vorausgesetzt - an der intersektoralen Neutralität der Ertragsbesteuerung nichts ändern, in intertemporaler Hinsicht aber sind wesentliche Unterschiede festzustellen. diese bestehen darin, daß infolge der Sofortabschreibung (anstelle einer Abschreibung pro rata temporis nach dem Ertragswertverfahren) mehr volkswirtschaftliches Vermögen an zukünftige Generationen weitergegeben wird. Hieraus aber den Schluß zu ziehen, daß das Mischsystem infolge dieser "Uberinvestition" zu Wohlfahrtsverlusten führt und deshalb unter Effiziengesichtspunkten abzulehnen ist, wäre falsch.

Wie wir anhand des einfachen graphischen Beispiels der Abb.2 zeigen konnten, ist das intertemporale Tauschoptimum in einer Welt ohne steuer dadurch gekennzeichnet, daß die Zeitpräferenzrate $\zeta$ der privaten Haushalte gleich ist der Grenzleistungsfähigkeit des akkumulierten volkswirtschaftlichen Vermögens. 
Im Rahmen unserer Analyse läßt sich dieses Tauschoptimum folgerichtig durch die Bedingung

(III.209)

$$
\zeta=r=\frac{m}{\mathrm{P}_{\mathrm{H}}}+\hat{\mathrm{P}}_{\mathrm{Hi}}-\alpha \delta \text {. }
$$

beschreiben.

Die SCHANZsche Einkommensteuer ist zwar investitionsneutral es gilt nach wie vor

(III.210)

$$
r=\frac{m}{\mathrm{P}_{\mathrm{H}}}+\hat{\mathrm{P}}_{\mathrm{H}}-\alpha \delta
$$

- sie treibt aber über die Besteuerung der Zinseinkünfte einen Keil zwischen den Marktzinssatz und die Zeitpräferenzrate der Haushalte: diese planen ihren Konsum nun derart, daß ihre zeitpräferenzrate mit dem Nettomarktzins übereinstimmt:

(III.211)

$$
\zeta=\theta \mathrm{e}^{\mathrm{r}}
$$

Die Haushalte werden durch die Zinseinkommensteuer dazu angeregt, zukünftigen Konsum durch gegenwärtigen zu substituieren, mit der Folge, daß sich die Bildung von Produktivvermögen und das Wirtschaftswachstum verlangsamen.

Diese intertemporale Allokationsverzerrung wird durch das Mischsystem neutralisiert: letzteres sorgt nämlich für eine Angleichung der Grenzleistungsfähigkeit des Produktivvermögens und der Zeitpräferenzrate der Haushalte; es gilt

( III.212)

$$
\zeta=\theta_{e} r=\frac{m}{\bar{P}_{H}}+\hat{p}_{H}-\alpha \delta
$$

Aus diesem Grunde haben wir das in Abschnitt III.5.1. vorgestellte SCHANZsche system als second-best-Lösung interpretiert das Mischsystem stellt die unter normativen Gesichtspunkten beste Lösung des untersuchten Besteuerungsproblems dar. 
Aber nicht nur, daB das Mischsystem unter normativen Gesichtspunkten positiv zu beurteilen ist, auch verwaltungstechnisch dürfte es einem System der Einkommensbesteuerung mit Abschreibungen pro rata temporis nach dem Ertragswerverfahren überlegen sein: es entfällt bei einer Sofortabschreibung der Anlagegüter nämlich die Notwendigkeit einer regelmäßigen Feststellung des Einheitswertes ertragbringender Vermögensbestände.

Darüberhinaus könnte man sich durch die Einführung des Mischsystems aller in Abschnitt III.5 aufgelisteten Probleme - die mit der Gewerbeertragsbesteuerung einhergehenden ausgenommen - in einem Zuge entledigen:

- der nicht-pekuniarisierbare Eigenleistungen erbringende Bauherr könnte nun kelne Vermögensvorteile mehr erzielen;

- alle Erwerbsformen von Wohnungseigentum wären unter Rentabilitätsgesichtspunkten als gleichwertig $z$ u beurteilen, und

- die Frage, ob Wertsteigerungen überhaupt und, wenn ja, zum Zeitpunkt ihrer Realisierung oder zum Zeitpunkt ihres Entstehens besteuert werden sollten, wäre ebenso gegenstandslos wie der Streit um die Frage, ob die "Konsumgut-" oder die "Investitionsgutlösung" die angemessenere Form der einkommensteuerlichen Behandlung selbstgenutzten Wohneigentums darstellt. 


\section{LITERATUR}

AARON, H. (1970): "Income Taxes and Housing",American Economic Review 60, s.789 ff.

ALBACH, H. (1970): Steuersysteme und unternehmerische Investitionspolitik, Wiesbaden.

ANDEL, N. (1980): "Einkommensteuer", in: F.NEUMARK (Hrsg.), Handbuch der Finanzwissenschaft, Band 2, Tübingen.

ANDREAE, C.-A. (1980): "Grundsteuern", in: F. NEUMARK (Hrsg.), Handbuch der Finanzwissenschaft, Band 2, Tübingen.

ARCELUS, F. und A.H. MELTzER (1973): "The Market for Housing and Housing Services", Journal of Money, Credit ard Banking 5, S.78 ff.

ARNOTT, R.J. (1979), "The Transition of Land to Urban Use", Journal of Political Economy 87, S. $161 \mathrm{ff}$.

ARROW, K.J. (1951): "An Extension of the Basic Theorems of Classical Welfare Economics", in: J. NEYMAN (Hrsg.), Proceedings of the Second Berkeley Symposium on Mathematical statistics on Probability, Berkeley und Los Angeles.

ARROW, K.J. und M. KURZ (1970), Public Investment and optimal Fiscal Policy, Baltimore.

ATKINSON, A.B. (1977): "Housing Allowances, Income Maintenance and Income Taxation", in: The International Economic Association (Hrsg.), The Economics of Public Services, Proceedings of a Conference held by the I.E.A. at Turin, S.3 ff., London.

AUERBACH, A.J. (1979): "Wealth Maximization and the Cost of Capital", Quarterly Journal of Economics 93, s.433 ff.

(1981): "Inflation and the Tax Treatment of Firm Behaviour, American Economic Review (Papers and Proceedings) $71, \mathrm{~s} .419 \mathrm{ff}$. 
(1982): "Tax Neutrality and the Social Discount Rate", Journal of Public Economics 17, S.355 ff.

BAILEY, M.J. (1959): "Formal Criteria for Investment Decisions", Journal of Political Economy 67, s.476 ff.

(1969): "Capital Gains and Income Taxation", in:

A.C. HARBERGER (Hrsg.), The Taxation of Income from Capital, S.11 ff., Washington.

BALLENTINE, J.G. (1978): "The Incidence of a corporation Income Tax in a Growing Economy, Journal of Political Economy 86, s.863 ff.

(1981): "The Cost of Intersectoral. and Intertemporel Price Distortions of a Corporate Income Tax", Southern Economic. Journal 48, s.87 ff.

BESSERER, K. (1984): "Intertemporale Fodenallokation und Besteuerung", in: H. SIEBERT (Hrsg.), Intertemporale Allokation, Frankfurt.

BICKEL, W. (1956): "Ertragsteuern", in: F. NEUMARK (Hrsg.), Handbuch der Finanzwissenschaft, Band 2 (2.Auflage), Tübingen.

BIEDENKOPF, K. und M. MIEGEL (1979), Wohnungsbau am wendepunkt. wohnungspolitik in der Sozialen Marktwirtschaft. Schriften des Instituts für Wirtschafts- und Gesellschaftspolitik (IWG), Band 2 (2.Auflage), Stuttgart.

BOADWAY, R.W. (1978): "Investment Incentives, Corporate Taxation, and the Efficiency in the Allocation of Capital", The Economic Journal 88, S.470 ff.

(1979), Public Sector Economics, Cambridge (Mass.). BÖHM-BAWERK, E.v. (1921), Kredit und Kapitalzins, Teil II: Positive Theorie des Kapitals, Band 1 (4.Auflage), Jena. BOSKIN, M:J. (1978): "Taxation, Saving and the Rate of Interest", Jounnal of Political Economy $86, \mathrm{~s} .3 \mathrm{ff}$. 
BOURNE, L.S. und J.R. HITCHCOCK (1978), Urban Housing Markets. Recent Directions on Research and Policy, Toronto.

BRADFORD, D. (1980): "Tax Neutrality and the Investment Tax Credit", in: H. AARON und M. BOSKIN (Hrsg.), The Economics of Taxation, S.281 ff., Washington.

BRADFORD, D. (1981): "The Incidence and Allocation Effects of a Tax on Corporate Distributiors", Jounnal of Public. Economics 15, s.1 ff.

BROWN, C. (1959): "Business Income Taxation and Investment Incentives", in: R. MUSGRAVE und C. SHOUP (Hrsg.), Readings in the Economics of Taxation, London. BRUECKNER, J.K., B. v. RABENAU (1981), "Dynamics of Land-Use for a Closed City", Regional Science and Urban Economics 11, S. 1 ff.

BRYANT, J. (1973): "Housing and Housing Policies in Western Europe, Kredit und Kapital 6, S.464 ff.

BUCHHOLZ, E. (1972), Wohnungswirtschaftliche Investitionsdeterminanten - eine theoretisch-empirische Analyse, Münster.

BUCKLEY, R., R. TUCCILLO und M. VILLANY (1977), Capital Markets and the Housing sector, Cambridge (Mass.).

BUND DER STEUERZAHLER (1984), "Zur Neuregelung der Eigenheimbesteuerung, Stellungnahmen des Karl-Bräuer-Instituts des Bundes der Steuerzahler" (Nr. 20).

BUNDESMINISTER DER FINANZEN (1976), Probleme und Lösungsmöglichkeiten einer Bodenwertzuwachsbesteuerung; Gutachten, erstattet vom Wissenschaftlichen Beirat beim BMF; Schriftenreihe, Heft 22, Bonn.

BUNDESMINISTER DER JUSTIZ (1981): "Zweites Gesetz zur Verbesserung der Haushaltsstruktur vom 22.12.1981", Bundesgesetzblott, Teil 1, Jahrgang 1981, s.1523 ff.

BUNDESMINISTER DER WIRTSCHAFT (1983), Probleme der wohnungswirtschaft. Gutachten des Wissenschaftlichen Beirats beim BMWi, Studienreihe 35, Bonn. 
BURNS, L. und I. GREBLER (1977), The Housing of Nations, London. CALVO, G.A., KOTLIKOFF, L.A., RODRIGUEz, D.A. (1979), "The Incidence of a Tax on Pure Rent: A New (?) Reason for an old Answer", Journal of Political Economy, S. $869 \mathrm{ff}$.

CHARLES, S. (1977), Housing Economics, Iondon, Basinstoke. COASE, R. (1937): "The Nature of the Firm", Economica 4, s.386 ff. CORDES, J. und S. SHEFFRIN (1981): "Taxation and the Sectoral Allocation of Capital in the U.S.", National Tax Journal 34 , S.419 ff.

DAVID, M. (1968), Alternative Approaches to Capital Gains Taxation, Washington.

DE LEEUW, F. (1971): "The Demand for Housing; a Review of CrossSection Evidence", Review of Economic Studies 53.

DESCHLER, U. (1983): "Bauträger- unci Bauherrenmodelle als unterschiedliche Formen der Investition in Immobilien", Der Betrieb 36, S.2491-2496 (Teil I), S.2543 - 2548 (Teil II).

DEUTSCHER BUNDESTAG (1981), Entwurf eines Gesetzes zur Stärkung der Investitionstätigkeit im Baubereich und zum Abbau ungleichmäBiger Besteuerung in der wohnungswirtschaft, Bundestag-Drucksache 9/843 v. 28.9.1981, Bonn.

DIEWERT, W. (1974): "Intertemporal Consumer Theory and the Demand for Durables", Econometrica 42, S.497 ff.

DORFMAN, R., P. SAMUELSON und R. SOLOW (1958), Linear Programming and Economic Analysis, New York, Toronto, London.

Downs, A. (1973), Federal Housing Subsidies: How Are They Working? Toronto, London.

EBNET, 0. (1978), Die Besteuerung des wertzuawchses. Ein theoretischer Ansatz im Rahmen der Einkommensbesteuerung, Baden-Baden.

ENGELS, F. (1923), Anti-Dühring, 12. Auflage, Berlin. 
ERNST, W. (1971): "Die Reform dés städtischen Bodenrechts als Aufgabe der Gesetzgebung", in: w. ERNST und w. BONCZEK (Hrsg.), Zur Reform des städtischen Bodenrechts, Hannover.

FAIR, R. (1972): "Disequilibrium in Housinc Models", Journal of Finance $27, \mathrm{~s} .207 \mathrm{ff}$.

FELDSTEIN, M. (1974): "Incidence of a Cap̧itāil Income Tax in a Growing Economy with Variable Saving Rates", Review of Economic Studies $41, \mathrm{~S} .505 \mathrm{ff}$.

FELDSTEIN, M. (1977), "The Surprising Incidence of a Tax on Pure Rent: A New Answer to an old Question", Journal of Political Economy 85, S. $349 \mathrm{ff}$.

(1978): "The Welfare Cost of Capital Income Taxation", Journal of Political Economy 86 (Sonderband), S. 29 ff.

FISHER, I. (1907), The Rate of Interest: It's Nature, Determination and Relation to Economic Phenomena, New York.

(1931), The Theory of Interest Rates as Determined by Impatience to spend Income and opportunity to Invest it, Wiederabdruck, New York.

FRIAUF, K., W. RISSE und K.-P. WINTERS (1978), Der Beitrag steuerlicher Maßnahmen zur Lösung der Bodenfrage, Schriftenreihe "städtebauliche Forschung" des Bundesministers für Raumordnung, Bauwesen und Städteplanung, Bad Godesberg.

FULLENKEMPER, H. (1982), Wirkungsanalyse der wohnungspolitik in der Bundesrepublik Deutschland. Beiträge zum Siedlungs- und Wohnungswesen und zur Raumplanung, Band 78, Münster.

FUISTING, B. (1907), Die PreuBischen direkten Steuern, I.Band: Kommentar zum Einkommensteuergesetz in der Fassung vom 19.Juni 1906, Berlin. 
GSCHWENDTNER, H. (1975): "Wirkung der Bodenwertzuwachssteuer auf Bodenpreise und Bodenspekulation", Jahrbücher ö̈r Nationalökonomie und Statistik, S.29 ff.

HAIG, R. (1921): "The Concept of Income - Economic and Legal Aspects", in: R. HAIG et al. (Hrsg.), The Federal Income Tax, New York.

HALL, R. und D. JORGENSON (1967): "Tax Policy and Investment Behaviour", American Economic Review 57, s.391 ff.

HAI:LER, H. (1973): "Grundsätzliches zur Besteuerung von Grundstücksgewinnen", Kredit und Kapital 6, S.255 ff.

(1981): Die Steuern - Grundlinien eines rationalen Systems öffentlicher Ausgaben, 3. neu bearbeitete Auflage, Tübingen.

HALLIWELL, J. (1969): "The Taxation of Capital Gains", Canadian Journal of Economics 2, s.314 ff.

HARBERGER, A. (1962): "The Incidence of the Corporation Income Tax", Journal of Political Economy 70, S.215 ff.

HAUSER, R., R. ULBRICH und G. ZITZELSBERGER (1978): "Distributive und fiskalische Wirkungen eines impliziten Transfers", Finanzarchiv (N.F.) 36 , s.393 ff.

HELLWIG, M. (1981): "Bankruptcy, Limited Liability and the Modigliani-Miller Theorem", American Economic Review 71 , S.155 ff.

HENDERSHOTT, P. (1980): "Real User Cost and the Demand for SingleFamily Housing", Brooking Papers on Economic Activity, $\mathrm{S} .402 \mathrm{ff}$.

HENDERSHOTT, P. und J. SLEMROD (1982), Taxes and the User cost of Capital for Owner-Occupied Housing, National Bureau of Economic Research, Cambridge (Mass.).

HENDERSON, J. und Y. IOANNIDES (1983): "A Model of Housing Tenure Choice". American Economic Review 73, s.98 ff. 
HEUER, J. (1983), "Bauherrenmodelle im sozialen Wohnungsbau. Studie einer Arbeitsgruppe", in: ökologische Forschungen. Schriftenreihe der Arbeitsgemeinschaft für Wohnungswesen, Stäciteplanung und Raumordnung an der Universität Bochum, Band 7, Bochum.

HIRSHLEIFER, J. (1974), Kapitaltheorie, Köln.

HOFFMANN, A. (1981): "Das unechte Zweifamileinhaus - eine einkommensteuerlich fragwürdige Einschränkung", Deutsche wohnungswirtschaft, $5.8 \mathrm{ff}$.

HOTELLING, H. (1931): "The Economics of Exhaustible Ressources", Journal of Political Economy 39, S.137 ff.

HUGHES, G. (1980): "Housing and the Tax System", in: G. HUGHES und G. HEAL (Hrsg.), Public Policy and the Tax system, London.

INTRILLIGATOR, M.D. (1971), Mathematical optimization and Economic Theory, Englewood Cliffs.

JAFFEE, D. (1972): "An Econometric Model of the Mortgage Market", in: M. GRAMLICH und D. JAFFEF, Savings, Deposits, Mortgages and Residential Construction, Lexington.

JATZEK, H. und W. LEIBFRITZ (1982), Der EinfluB der Steuern aú die Investitionstätigkeit der unternehmen, Berlin und München.

JOHANSSON, S.-E. (1961), skatt - Investering - Värdering, stockholm. JORGENSON, D. (1963): "Capital Theory and Investment Behaviour", American Economic Review (Papers ard Proceedings) 53, S. $247 \mathrm{ff}$.

(1967): "The Theory of Investment Behaviour", in: R. FERBER (Hrsg.), Determinants of Investment Behaviour, New York. 
KAMIEN, M. und N. SCHWARTZ (1981), Dynamic Optimization. The Calculus os Variations and Optimal Control in Economics and Management, Amsterdam.

KAY, J. und M. KING (1978), The British Tax System, Oxford.

KIEFER, D. (1981): "The Interaction of Inflation and the U.S. Tax Subsidies on Housing", National Tax Journal 34, S. $433 \mathrm{ff}$.

KING, A. (1977): "The Demand for Housing", Southern Economic Journal 43, S.1077 ff.

KIRMSE, K. (1979): "Nutzung und Veräußerung von Grundbesitz als private Vermögensverwaltung oder gewerbliche Tätigkeit", in: SCHONHOFER und REINISCH, Haus- und. Grundbesitz in Recht und Praxis, Band III, Gruppe 15, S.76! ff., Freiburg.

LAIDLER, D. (1969): "Income Tax Incentives for Owner-Occupied Housing", in: A. HARBERGER und M. BAILEY (Hrsg.), The Taxation of Income from Capital, Washington.

LANGE, O. (1942): "The Foundations of Welfare Economics, Econometrica 10, S.215 ff.

LANGE, O. und F. TAYLOR (1948), On the Economic Theory of Socialism, 2. Auflage, Minneapolis.

LEE, T. (1968): "Housing and Permanent Income", Review of Economic Studies 50 .

LITZENBERGER, R. und H. SOSIN (1978): "Taxation and the Incidence of Homeownership Across Income Groups, Journal of $\mathrm{Fi}$ nance $33, \mathrm{~S} .947 \mathrm{ff}$.

MACKSCHEID, K. und W. DEICHMANN (1982), Zur Leistungsfähigkeit von Subventionen in der Wohnungswirtschaft: Effizienzaralyse allokativer und. distributiver Effekte staatlicher Transfers für die Wohnungswirtschaft, Frankfurt.

MAISEL, S. (1963): "A Theory of Fluctuations in Residential Construction Starts", American Economic Review 53, S. 359 ff. 
MALINVAUD, E. (1953): "Capital Accumulation and Efficient Allocation of Resources", Econometrica 21, s.233 ff.

(1962): "Efficient Capital Accumulation - Corrigendum", Econometrica 30, S.570 ff.

MAYO, S. (1981): "Theory and Estimation in the Economics of Housing Demand", Journal of Urban Economics 10, s.95 ff.

MEADE-COMMITTEE (1978), The Structure and Reform of Direct Taxation. Report of a Committee chaired by Prof. J. MEADE, London, Boston, Sidney.

MELTZER, A. (1974): "Credit Availability ar:d Economic Decisjons", Journal of Finance $29,5.263 \mathrm{ff}$.

MILLER, M. (1977): "Debt and Taxes", Jounnal of Finance 32, S.261 ff. MILLER, M. und F. MODIGLIANI (1958): "The Cost of Capital, Corporation Finance and the Theory of Investment", Amenican Economic Review 48, S.259 ff.

MöLlER, H. (1967), Der Boden in der politischen ökonomie, Wiesbaden.

MOORHOUSE, J.C. (1972): "Optimal Housing Maintenance under Rent Control", Southern Economic Journal $34, \mathrm{~S} .93 \mathrm{ff}$.

MUtLLER, H. (1953), Die städtische Grundrente und die Bewertung von Baugrundstücken, Tübingen.

MUSGRAVE, R., P. MUSGRAVE und L. KULLMER (1977), Die öffentlichen Finanzen in Theorie und Praxis, Tübingen.

MUTH, R. (1960): "The Demand for Non-Fc.rming Housing", in:

A. HARBERGER (Hrsg.), The Demand for Duratile Goods, Chicago.

NACHTKAMP, H.H. (1984a): "Grundsätze der Besteuerung, steuerlicher Einkommensbegriff und Konsequenzen für die steuerliche Behandlung des Wohnungsbaus, insbesondere des selbstgenutzten Wohneigentums", in: XIX. Königsteiener Gespräche, Schriftenreihe des Instituts für städtebau Wohnungswirtschaft und Bausparwesen (Arnold-KnoblauchInstitut) e.v., Bonn. 
NACHTKAMP, H.H. (1984b): "Das Für und Wider staatlicher Interventionen in den Wohnungsbau und die Wohnversorgung", in: H. SIEBERT (Hrsg.), Intertemporale Allokation, Frankfurt.

NELL-BREUNING, O.V. (1956): "Erfassung der steigenden Grundrente", wirtschaft und Gesellschaft, Band I, Freiburg.

(1970): "St:euern als Instrument.e der Bodenpolitik", in: H. HALLER (Hrsg.), Theorie und Praxis des finanzpolitischen Interventionismus, Tübingen.

(1972): "Steuerrechtliche Vorschläge zur Erfassung des Bodenwertzuwachses", Bundesbaublatt, S. $459 \mathrm{ff}$.

OLSEN, E. (1969): "A Competitive Theory of the Housing Market", American Economic Review 59, S.612 ff.

(1971): "Subsidized Housing in a Competitive Market", American Economic Review 61, s.220 ff.

PABST, G. (1983): "Bauherrenmodell und Grunderwerbsteuer nach dem GrEStG 1983 und nach der neuen BFH-Rechtssprechung", Der Betrieb 36, s.849 ff.

PAHLKE, J. (1980): "Verkehrsteuern", in: W. ALBERS und andere (Hrsg.), Handwörterbuch der wirtschaftswissenschaften, stuttgart, New York.

PAGE, T. (1977), Conservation aná Economic Efficiency. An Approach to Materials Policy, Baltimore.

PETZOLD, G. (1983), Gewerbesteuer, München.

PFEIFFER, U. (1972): "Ansätze für eine bodenpolitische Konzeption", in: R. THOSS (Hrsg.), Bodenordnung und Bodenpolitik, Münster.

(1982): "Eigentumsbildung im Abwind", Privates Bausparwesen, S.55 ff.

QUAST, D. (1983): "Das Bauherrenmodell - ein steuerpolitisches Ärgernis?", Der Betrieb, S.2113 ff. 
RABENAU, B.v. (1973), Urban Dynamic Models, Ph.D. Dissertation, Berkely.

REICHSFINANZMINISTERIUM (1937): "Verordnung über die Bemessung des Nutzungswertes der Wohnung i.m eigenen Einfamilienhaus vom 26.1.1937", Reichssteuerblatt, 2.2.1937, S.161 ff.

RICARDO, D. (1905), Grundsätze der Volkswirtschaft und Besteuerung, deutsche tubersetzung von O. THIELE, Jena.

RISSF, W. (1974), Grundzüge einer Theorie des Baubodenmarktes, Bonn.

ROBINSON, R. (1979), Housing Economies and Public Policy, London. ROGGE, C. (1937): "Die steuerliche Behandlung des Nutzungswertes der Wohnung im eigenen Haus", Deutsche Steuerzeitung und Wirtschaftlicher Beobachter 27, s.610 ff.

ROLPH, E. (1983) "Discriminating Effects of the Income Tax Treatment of Owner-Occupants", National Tax Journal 36, $S .471 \mathrm{ff}$.

ROSE, G. (1978), Betrieb und Steuern - Erstes Buch: Die Ertragsteuern, wiesbaden.

- (1982), Betrieb und Steuern - Drittes Buch: Die Substanzsteuern, Wiesbaden.

ROSS, F.-W. und R. BRACHMANN (1983), Ermittlung des Bauwertes von Gebäuden, 24. Auflage, Hannover.

SACHVERSTÄNDIGENRAT (1981/82): Sachverständigenrat zur Begutachtung der gesamtwirtschaftlichen Entwicklung, Investieren für mehr Beschäftigung, stuttgart.

SANDMO, A. und A. ATKINSON (1980): "Welfare Implications of the Taxation of Savings", Economic Journal 90, S.529 ff.

SCHANZ, G. (1886): "Der Einkommensbegriff und die Einkommensteuergesetze", Finanzarchiv 13, S.1 ff.

SCHNEIDER, D. (1974), Investition und Finanzierung, Lehrbuch der Investitions-, Finanzierungs- und Unçewißheitstheorie, Opj.aden. 
SCHNEIDER, H.-K. und R. KORNEMANN (1977), Soziale Wohnungsmanktwirtschaft, Bonn.

SCHNEIDER, O. (1982): "Die neue Politik fü das Wohnungseigentum", Privates Bausparwesen, $5.41 \mathrm{ff}$.

SCHÖNHOFER/REINISCH (1985), Haus und Grundbesitz in Recht und Praxis, Freiburg.

SCHREIBER, U. (1984), Rechtsformabhängige unternehmensbesteuerung?Eine Kritik des Verhältnisses von Einkommen- und Körperschaftsteuer auf der Grundlage eines Modells für mehrperiodige Steuerwirkungsanalysen, Habilitationsschrift, Mannheim.

SCHULZ, H.-J. (1982), Der EinfluB der Besteuerung auf Marktaktivitäten und: Marktproduktion eines Monopolisten bei unvollkommenem Kapitalmarkt - Ein Beitrag zur Dynamisierung der Steuerüberwälzungsanalyse, Frankfurt am Main.

SELCHERT, F. (1982): "Einführung der Pauschalbesteuerung für eigengenutzte Zwei- und Mehrfamilienhäuser - ein Irrweg", Betriebsberater, $\mathrm{S.607}$ ff.

SHELTON, J.P. (1968): "The Cost of Renting Versus Owning a Home", Land Economics 14, S.59 ff.

SHOUP, C. (1970): "The White Paper: Accrual Accounting for Capital Gains and Losses", Canadian Tax Jour.nal 18, s.96 ff.

SIMONS, H.C. (1938), Personal Income Taxation, Chicago und London. SINN, H.W. (1982), Besteuerung, wachstum und Kapitalstruktur (Habilitationsschrift), Mannheim.

(1984): "Das Problem der Baulücken - eine allokationstheoretische Untersuchung zur Funktionsweise des Baumarktes und zu den Möglichkeiten seiner Regulierung", Schriften des vereins für Socialpolitik, Gesellschaft für wirtschafts - und Sozialwissenschaften, Neue Folge, Band 140, S.339 ff. 
SMITH, B. (1976): "The Supply of Urban Housing", Quarterly Journal of Economics 90, s. ff.

SMITH, L. (1969): "A Model of the Canadian Housing and Mortgage Markets", Journal of Political Economlj 77, s.795 ff.

STEINER, J. (1980), Gewinnsteuern in Partialmodellen für Investitionsentscheidungen - Barwert und Endwert als Instrumente dir Steuerwirkungsanclyse, Münster.

STIGLITZ, J. (1981): "Pareto-Optimality and Competition", Journal of Finance $36, \mathrm{~S} .235 \mathrm{ff}$.

STREIT, M. (1979), Theorie der Wirtschaftspolitik, Düsseldorf. STUHRMANN, G. (1982): "Die steuerlichen Maßnahmen auf dem Gebiet des Wohnungsbaus und der Wohnungsmodernisierung durch das 2. Haushaltsstrukturgesetz", Betriebsberater, S. $107 \mathrm{ff}$.

TEWES, D. (1980), Praktische Auswirkungen des neuen BFH-urteils zur steuerlichen Anerkennung von Bauherrenmodellen, Kissing.

TIMM, H. (1980): "Steuervergünstigungen und Transferzahlungen als Instrumente der staat.lichen Allokations- und Stabilitätspolitik - ein kritischer Verg?.eich", in: H. SrEBERT, (Hrsg.), Politik und Markt, stutt.gart und New York. (1981): "Finanzwirtschaftliche Allokationspolitik", in: F. NEUMARK (Hrsg.), Handbuch der Finanzwissenschaft, Band 3, Tübingen.

TROLL, M. (1972), Grund und Boden. Politik und Steuern, Heidelberg.

TULLOCK, G. (1971): "Subsidized Housing in a Competitive Market; Comment", American Economic Review 61, s.218 ff.

VANDELL, K. (1982): "Towards a Model of Credit Rationing and Term Adjustment in the Mortgage Market", in: C. SIRMANS (Hrsg.), Research in Real Estate 1, Toronto. 
VOSGERAU, H.-J. (1972): "Boden und wirtschaftliches Wachstum", Kyklos, S. $481 \mathrm{ff}$.

WASSERFALLEN, W. (1979): "Wohnungsmarkt und Kreditmärkte", Schuieizerische Zeitschrift für Volkswirtschaft und statistik, S.139 ff.

WEISS, J. (1978): "Capital Gains, Discriminatory Taxes and the Choice Between Renting and Owning a House", Journal of Public Economics 10, s.45 ff.

WHITF, M. und L. WHITE (1977): "The Tax Subsidy to Owner-Occupied Housing: Who Benefits?", Journal of Public Economics 7, S. $111 \mathrm{ff}$.

WILLMS, M. (1982), Wohnungswirtschaftliche und fiskalische wirkungen des Bauherrenmodells, München.

WINKELMANN, W. (1985), Staatliche Einflußnahme auf die Wohnungsversorgung. Motive, Formen und Wirkungen, Köln. 


\section{STAATLICHE ALLOKATIONSPOLITIK IM MARKTWIRTSCHAFTLICHEN SYSTEM}

Band 1 Horst Siebert (Hrsg.): Umweltallokation im Raum. 1982.

Band 2 Horst Siebert (Hrsg.): Global Environmental Resources. The Ozone Problem. 1982.

Band 3 Hans-Joachim Schulz: Steuerwirkungen in einem dynamischen Unternehmensmodell. Ein Beitrag zur Dynamisierung der Steuerüberwälzungsanalyse. 1981.

Band 4 Eberhard Wille (Hrsg.): Beiträge zur gesamtwirtschaftlichen Allokation. Allokationsprobleme im intermediären Bereich zwischen öffentlichem und privatem Wirtschaftssektor. 1983.

Band 5 Heinz König (Hrsg.): Ausbildung und Arbeitsmarkt. 1983.

Band 6 Horst Siebert (Hrsg.): Reaktionen auf Energiepreissteigerungen. 1982.

Band 7 Eberhard Wille (Hrsg.): Konzeptionelle Probleme öffentlicher Planung. 1983.

Band 8 Ingeborg Kiesewetter-Wrana: Exporterlösinstabilitat. Kritische Analyse eines entwicklungspolitischen Problems. 1982.

Band 9 Ferdinand Dudenhöffer: Mehrheitswahl-Entscheidungen über Umweltnutzungen. Eine Untersuchung von Gleichgewichtszuständen in einem mikroökonomischen Marktund Abstimmungsmodell. 1983.

Band 10 Horst Siebert (Hrsg.): Intertemporale Allokation. 1984.

Band 11 Helmut Meder: Die intertemporale Allokation erschópfbarer Naturressourcen bei fehlenden Zukunftsmärkten und institutionalisierten Marktsubstituten. 1984.

Band 12 Ulrich Ring: Öffentliche Planungsziele und staatliche Budgets. Zur Erfüllung öffentlicher Aufgaben durch nicht-staatliche Entscheidungseinheiten. 1985.

Band 13 Ehrentraud Graw: Informationseffizienz von Terminkontraktmärkten für Währungen. Eine empirische Untersuchung. 1984.

Band 14 Rüdiger Pethig (Ed.): Public Goods and Public Allocation Policy. 1985.

Band 15 Eberhard Wille (Hrsg.): Öffentliche Planung auf Landesebene. Eine Analyse von Planungskonzepten in Deutschland, Österreich und der Schweiz. 1986.

Band 16 Helga Gebauer: Regionale Umweltnutzungen in der Zeit. Eine intertemporale ZweiRegionen-Analyse. 1985.

Band 17 Christine Pfitzer: Integrierte Entwicklungsplanung als Allokationsinstrument auf Landesebene. Eine Analyse der offentlichen Planung der Lander Hessen, Bayern und Niedersachsen. 1985.

Band 18 Heinz Konig (Hrsg.): Kontrolltheoretische Ansatze in makroökonometrischen Modellen. 1985.

Band 19 Theo Kempf: Theorıe und Empırıe betrieblıcher Ausbildungsplatzangebote. 1985.

Band 22 Bernd Gutting: Der Einfluß der Besteuerung auf die Entwicklung der Wohnungs- und Baulandmarkte. Eine intertemporale Analyse der bundesdeutschen Steuergesetze. 1986. 
